\title{
Geminal Diazides Derived from 1,3-Dicarbonyls: A Protocol for Synthesis
}

Hellmuth Erhardt, ${ }^{1}$ Andreas Häring, ${ }^{1}$ Andreas Kotthaus, ${ }^{1}$ Markus Roggel, ${ }^{1}$ My Linh Tong, ${ }^{1}$

Phillip Biallas, ${ }^{1}$ Martin Jübermann, ${ }^{1}$ Fabian Mohr, ${ }^{2}$ Stefan F. Kirsch ${ }^{1 *}$

${ }^{1}$ Organic Chemistry, Bergische Universität Wuppertal, Gaußstr. 20, 42119 Wuppertal, Germany

${ }^{2}$ Inorganic Chemistry, Bergische Universität Wuppertal, Gaußstr. 20, 42119 Wuppertal, Germany

\section{Supporting information}

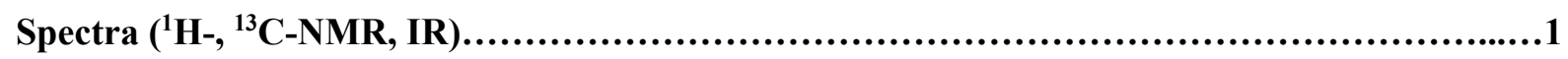

Crystallographic data...................................................................67

TGA-DSC measurements..............................................................73

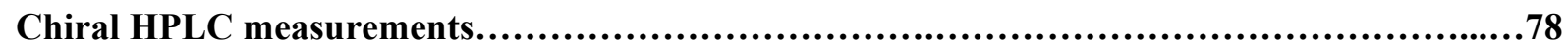


Spectra $\left({ }^{1} \mathrm{H}-\mathrm{NMR},{ }^{13} \mathrm{C}-\mathrm{NMR}, \mathrm{IR}\right)$

Dimethyl 2,2-diazidomalonate (2a)

${ }^{1} \mathrm{H} \mathrm{NMR}(600 \mathrm{MHz}, \mathrm{CDCl})$
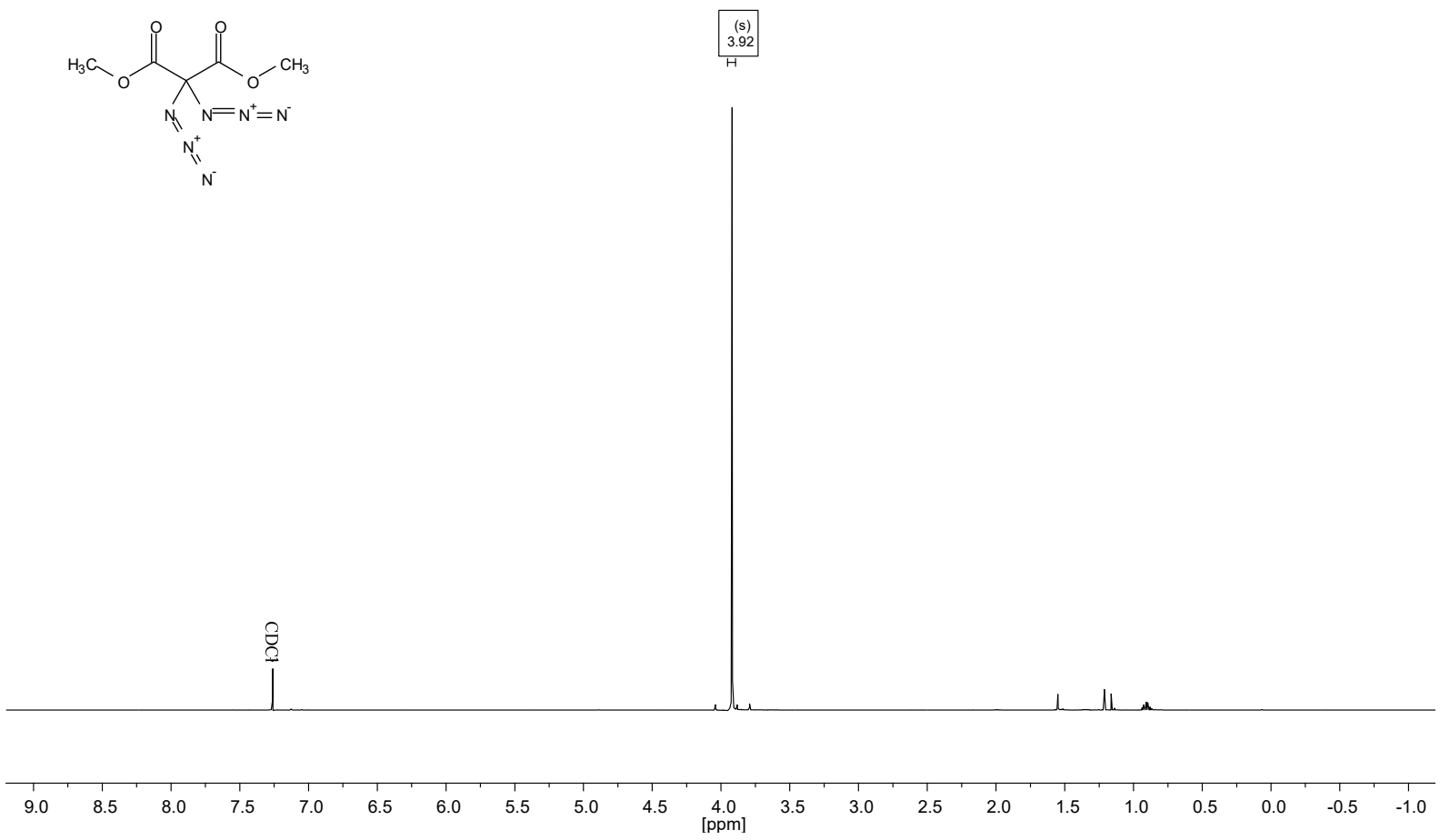

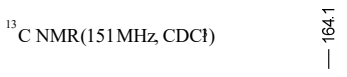

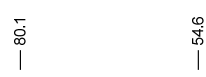
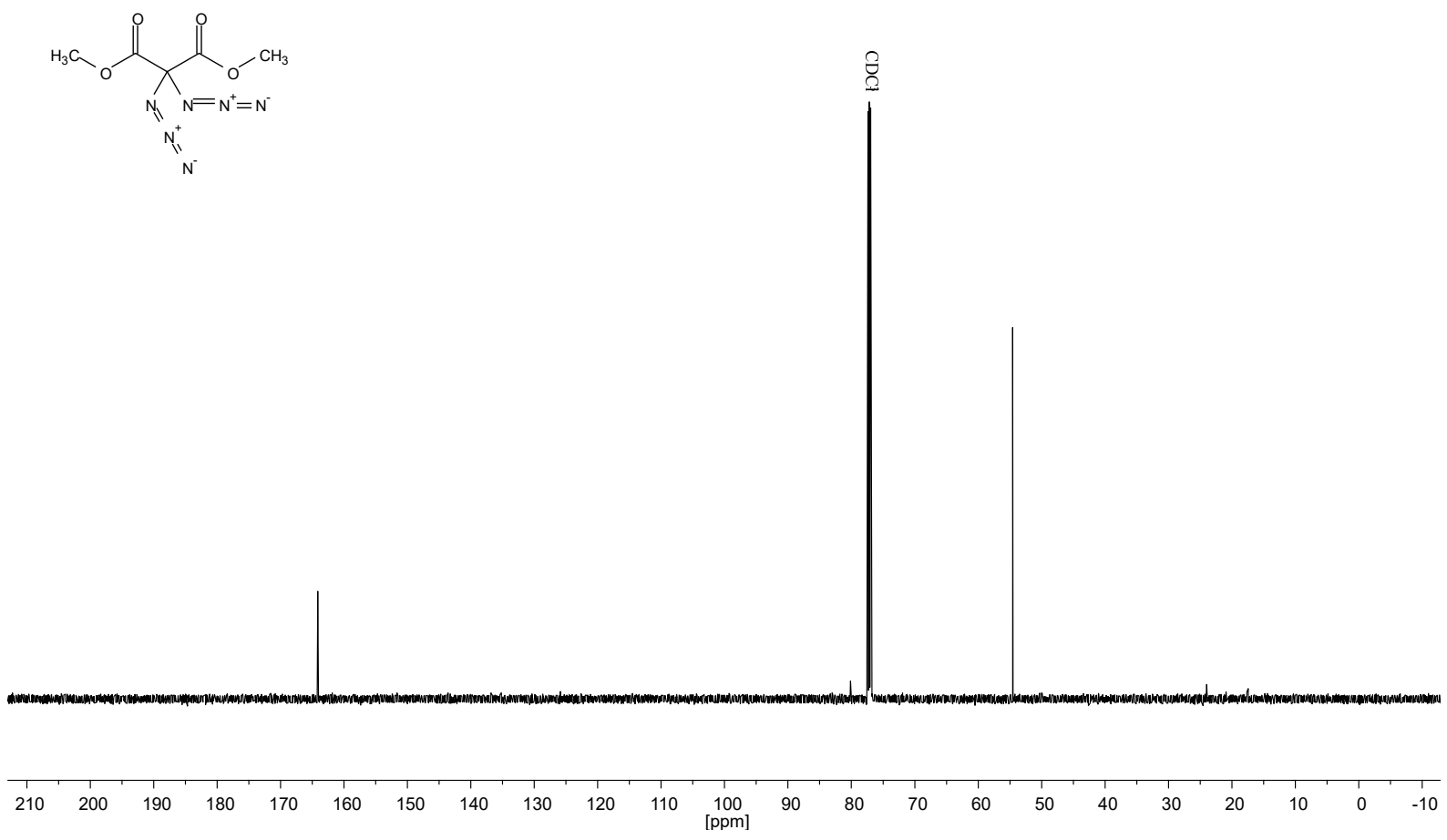

1 


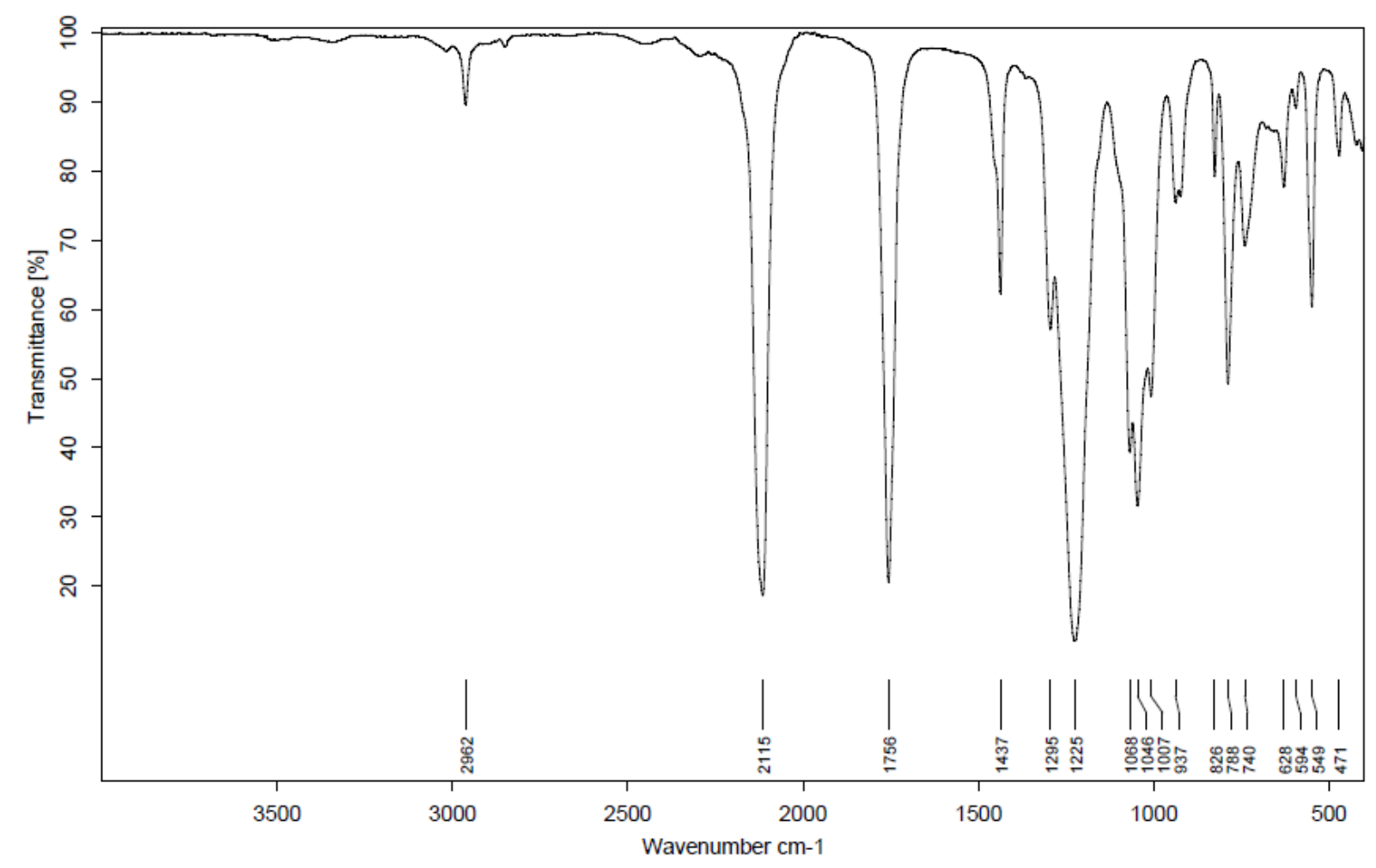

Dimethyl 2,2-bis(4-phenyl-1H-1,2,3-triazol-1-yl)malonate

${ }^{1} \mathrm{H} \mathrm{NMR}(400 \mathrm{MHz}, \mathrm{CDCl})$
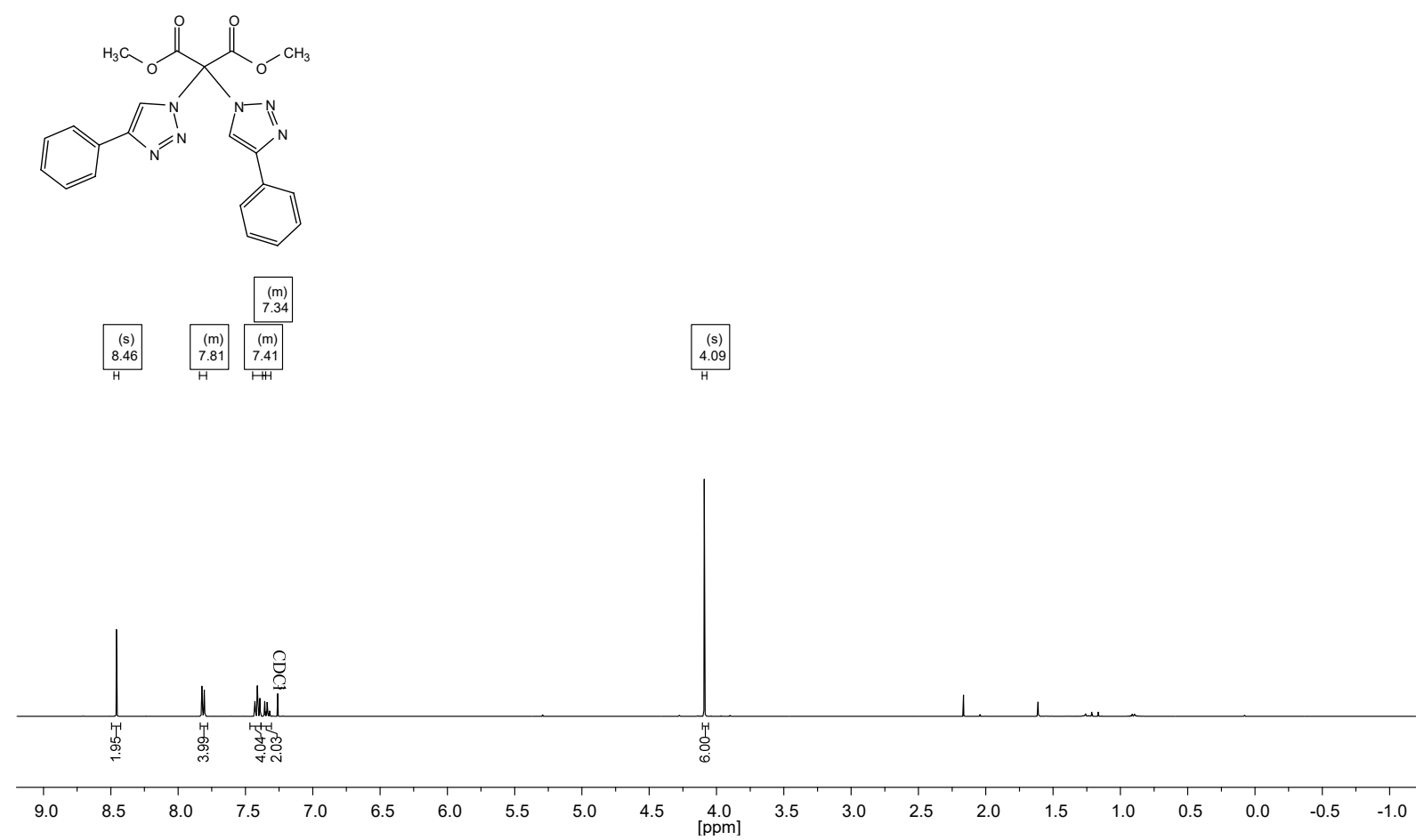

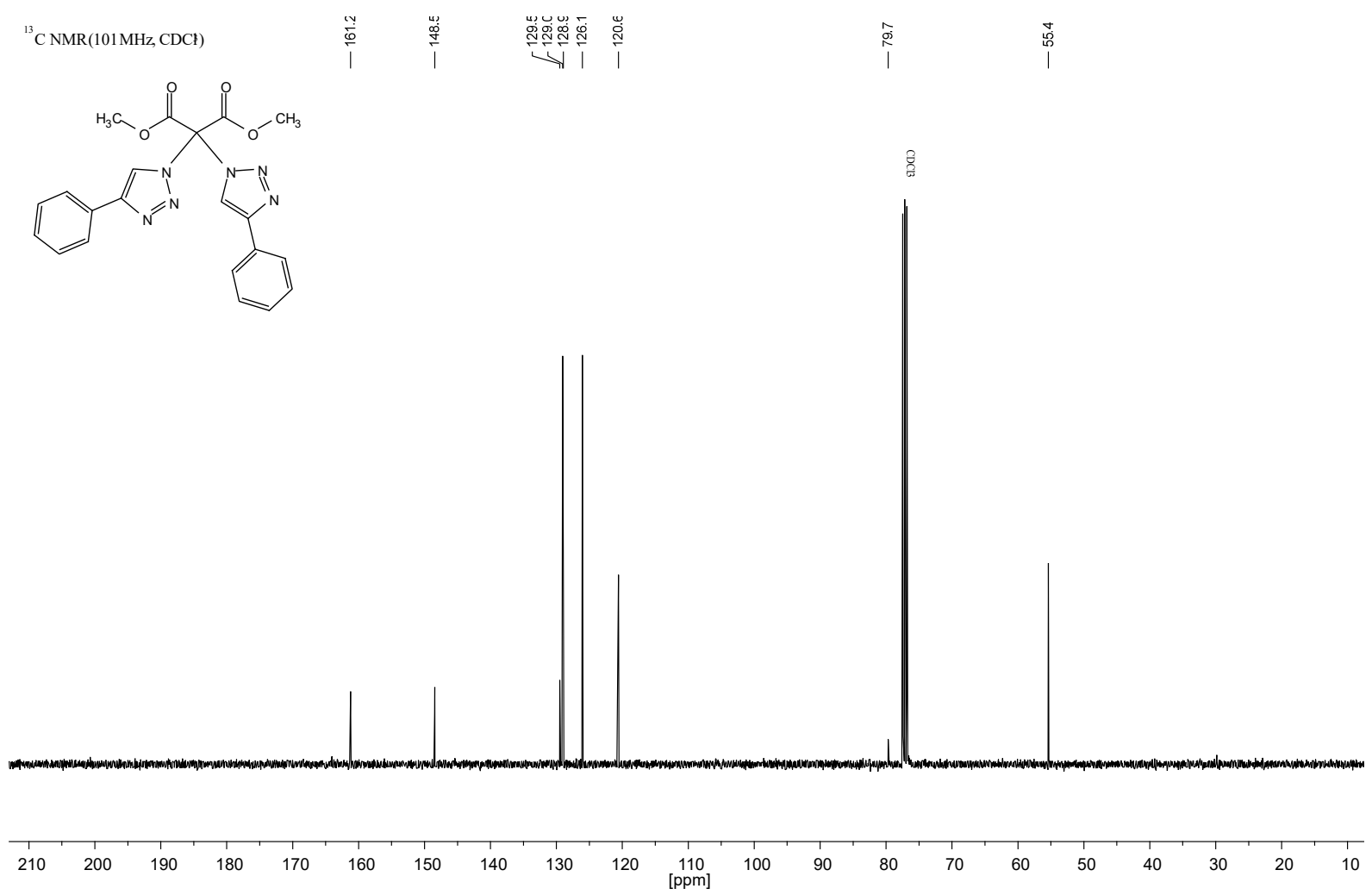

Diethyl 2,2-diazidomalonate (2b)

${ }^{1} \mathrm{H}$ NMR(600MHz, CDCl)

\begin{tabular}{|r|}
\hline$(m)$ \\
4.38 \\
\hline-
\end{tabular}

\begin{tabular}{|r|}
\hline$(\mathrm{m})$ \\
1.36 \\
\hline$H$
\end{tabular}
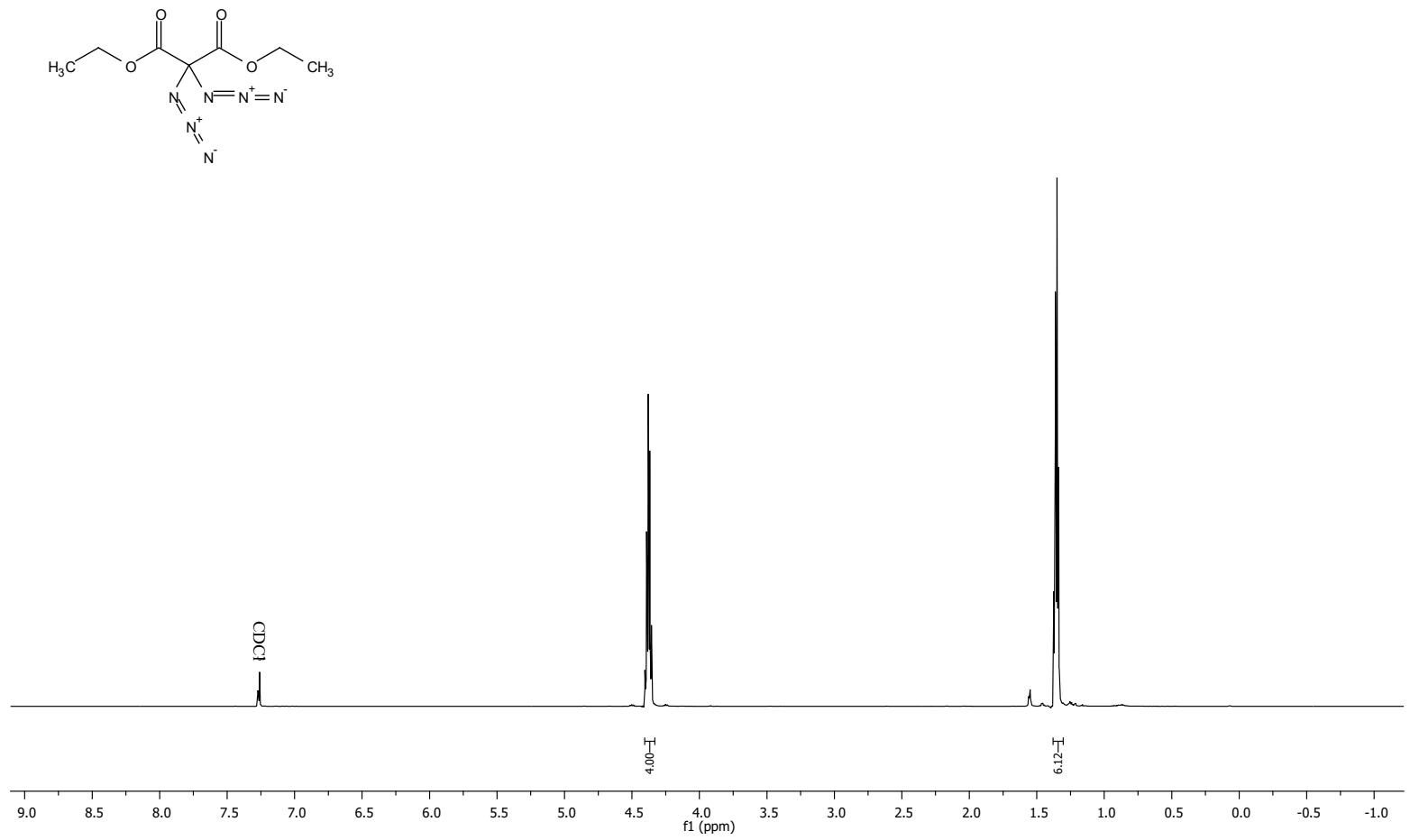

3 
${ }^{13} \mathrm{CNMR}(151 \mathrm{MHz}, \mathrm{CDCl})$

$\underbrace{O}_{\substack{N_{N}=N^{+}=N^{-} \\ N_{N}^{+}}}$
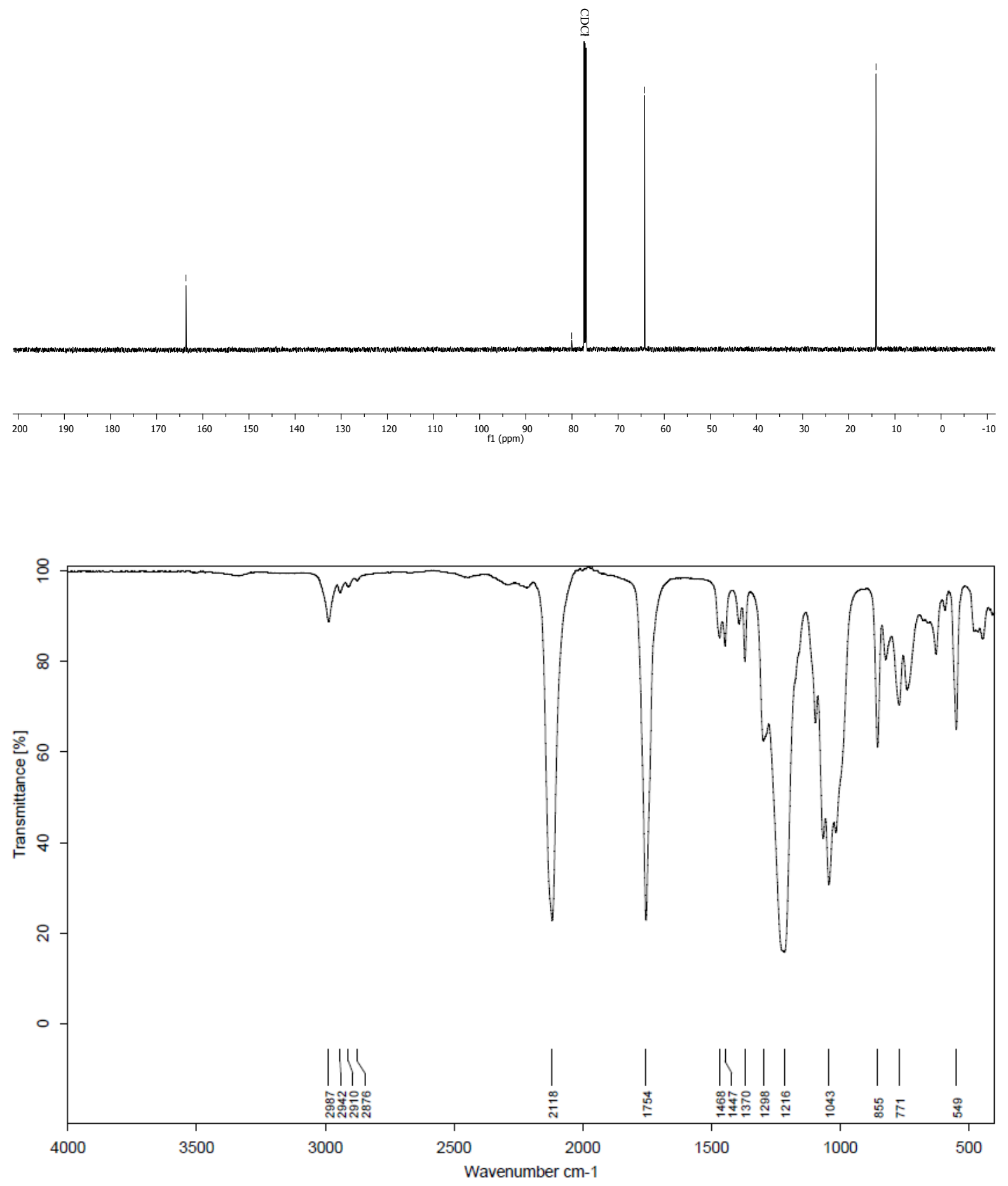
Di-tert-butyl 2,2-diazidomalonate (2c)

${ }^{1} \mathrm{H}$ NMR (400MHz, CDCl)

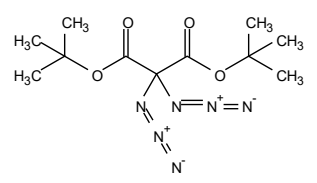

\begin{tabular}{|r|}
\hline$(s)$ \\
1.54 \\
\hline$H$
\end{tabular}

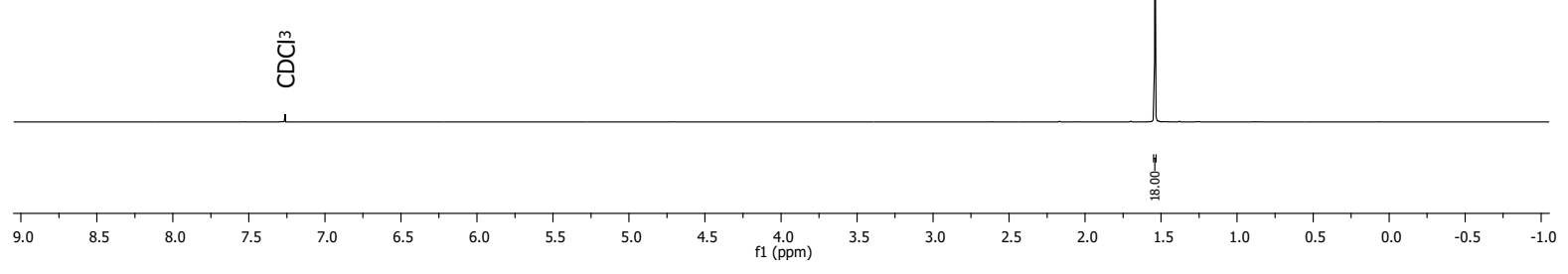

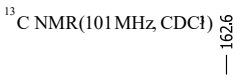

\begin{tabular}{ll}
$\overrightarrow{0}$ & \multirow{0}{\infty}{} \\
1 & $\mid$
\end{tabular}

$\stackrel{9}{i}$
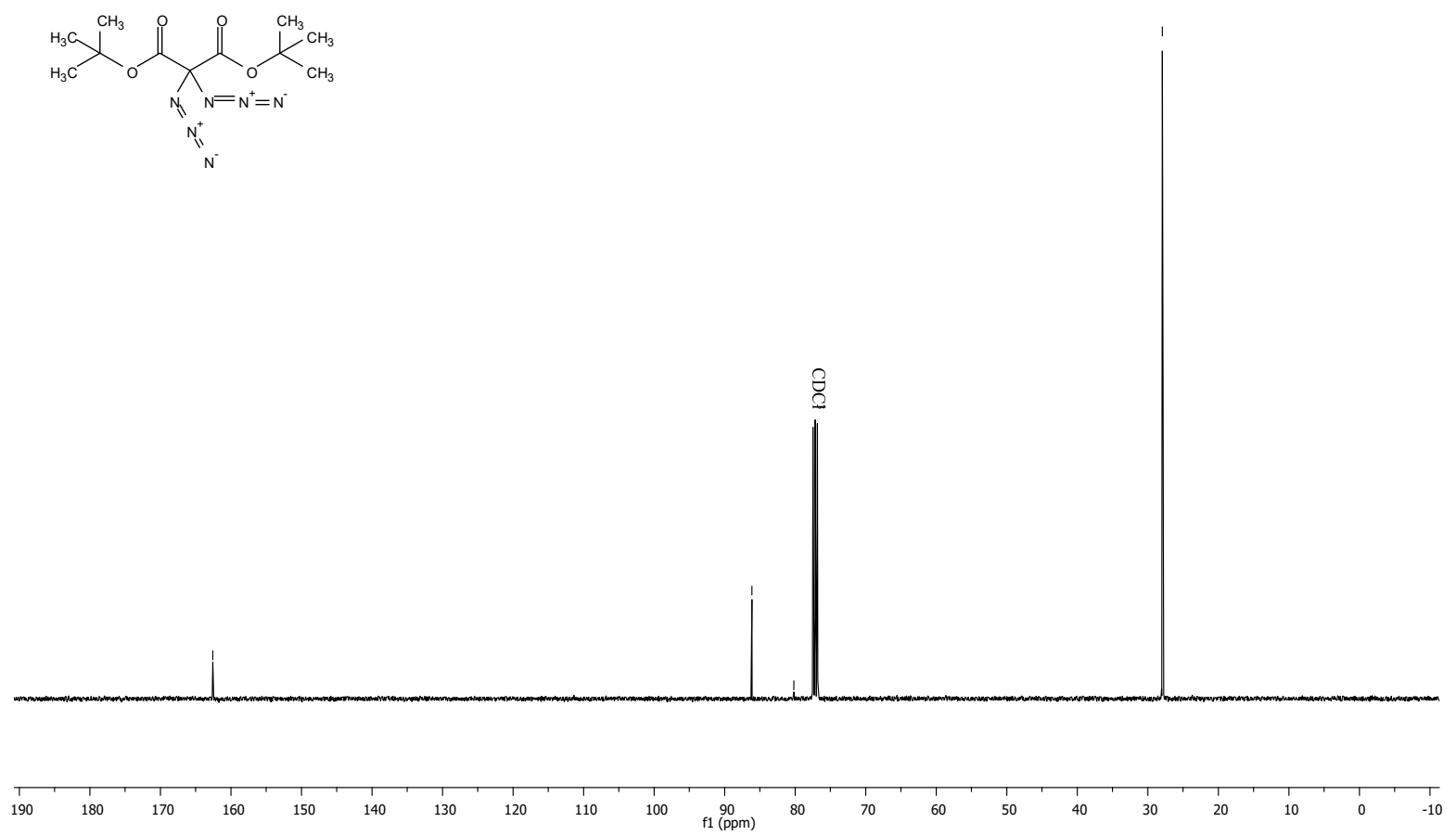

5 


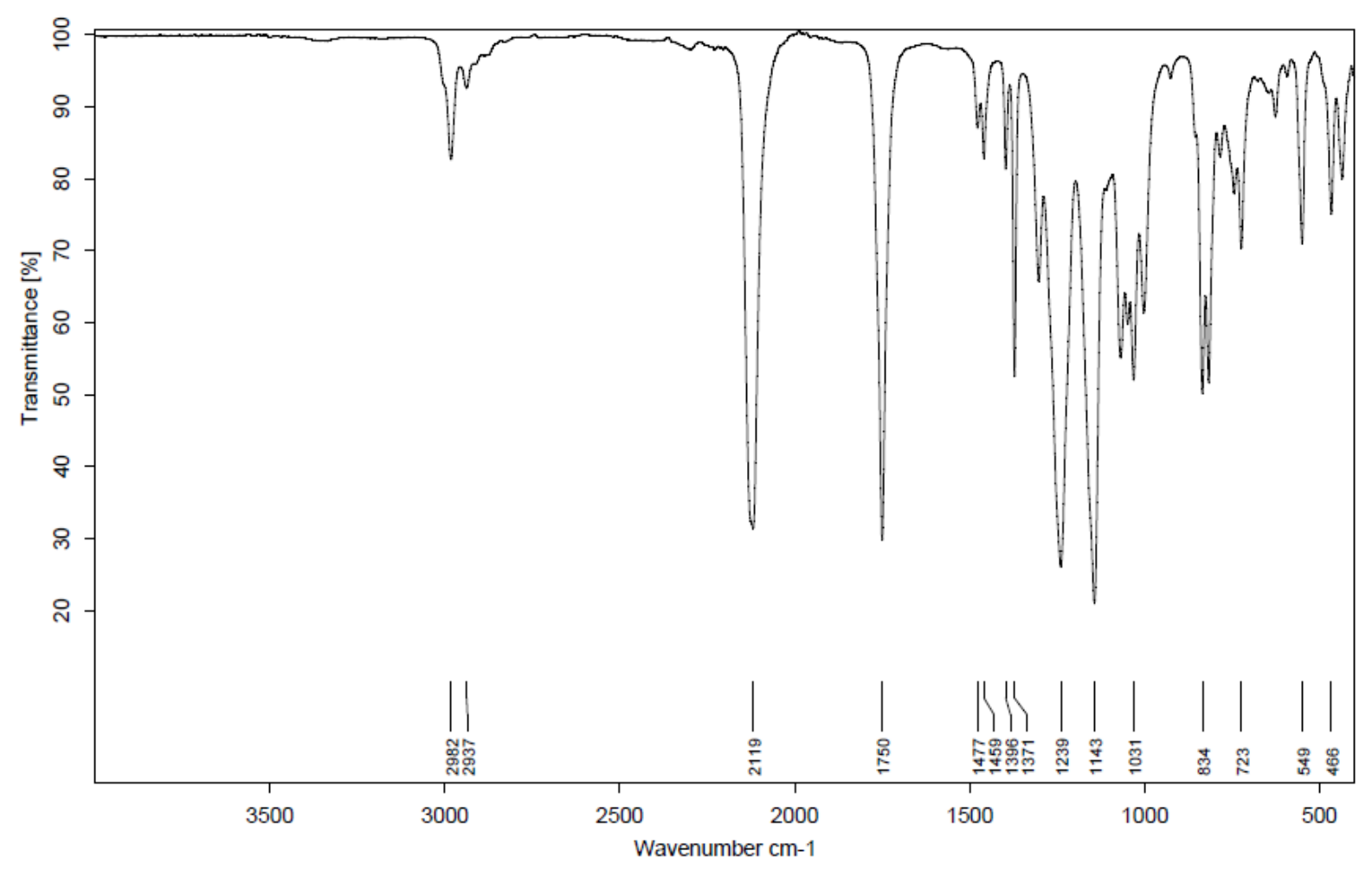

Diallyl 2,2-diazidomalonate (2d)

${ }^{1} \mathrm{H} \mathrm{NMR}(400 \mathrm{MHz}, \mathrm{CDC})$
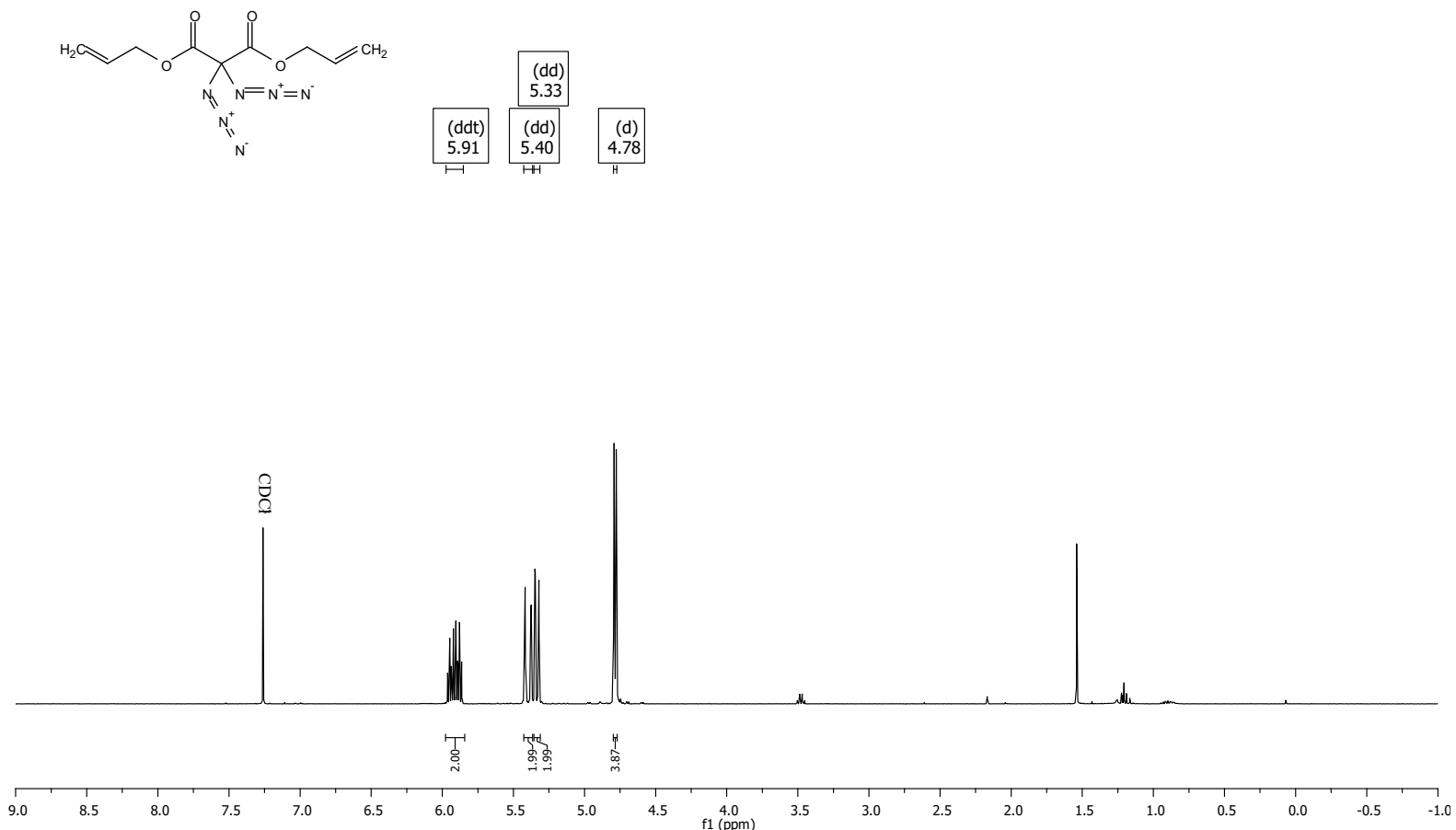
${ }^{13} \mathrm{C} \mathrm{NMR}(101 \mathrm{MHz}, \mathrm{CDC}){ }_{\tilde{0}}^{+}$
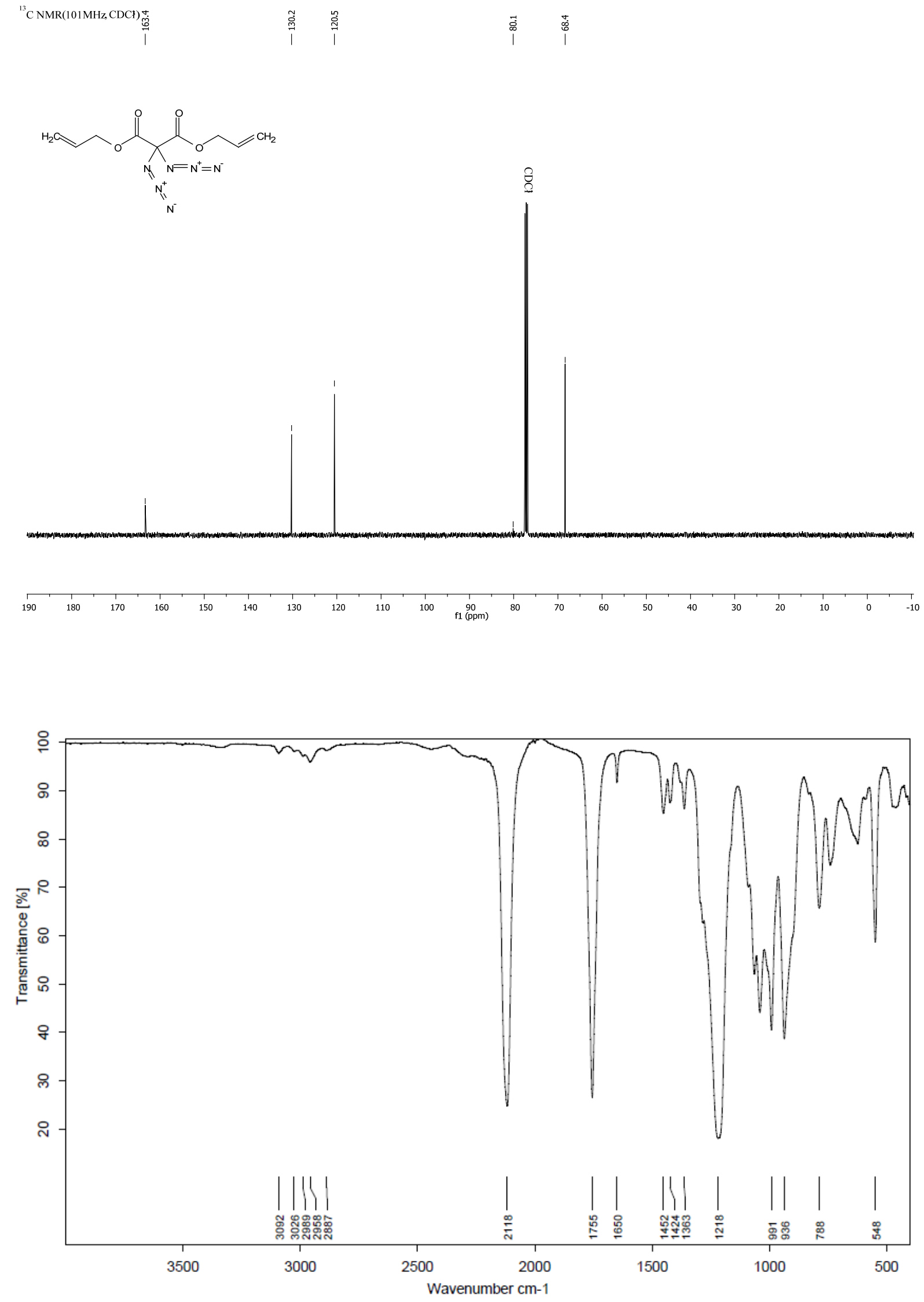
Methyl 2,2-diazido-3-oxobutanoate (2e)

${ }^{1} \mathrm{H} \mathrm{NMR}(600 \mathrm{MHz}, \mathrm{CDCl})$

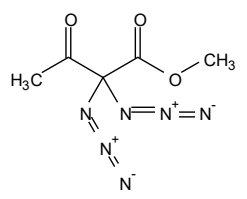

\begin{tabular}{r|}
\hline$(s)$ \\
3.90 \\
\hline
\end{tabular}

$(s)$
2.26

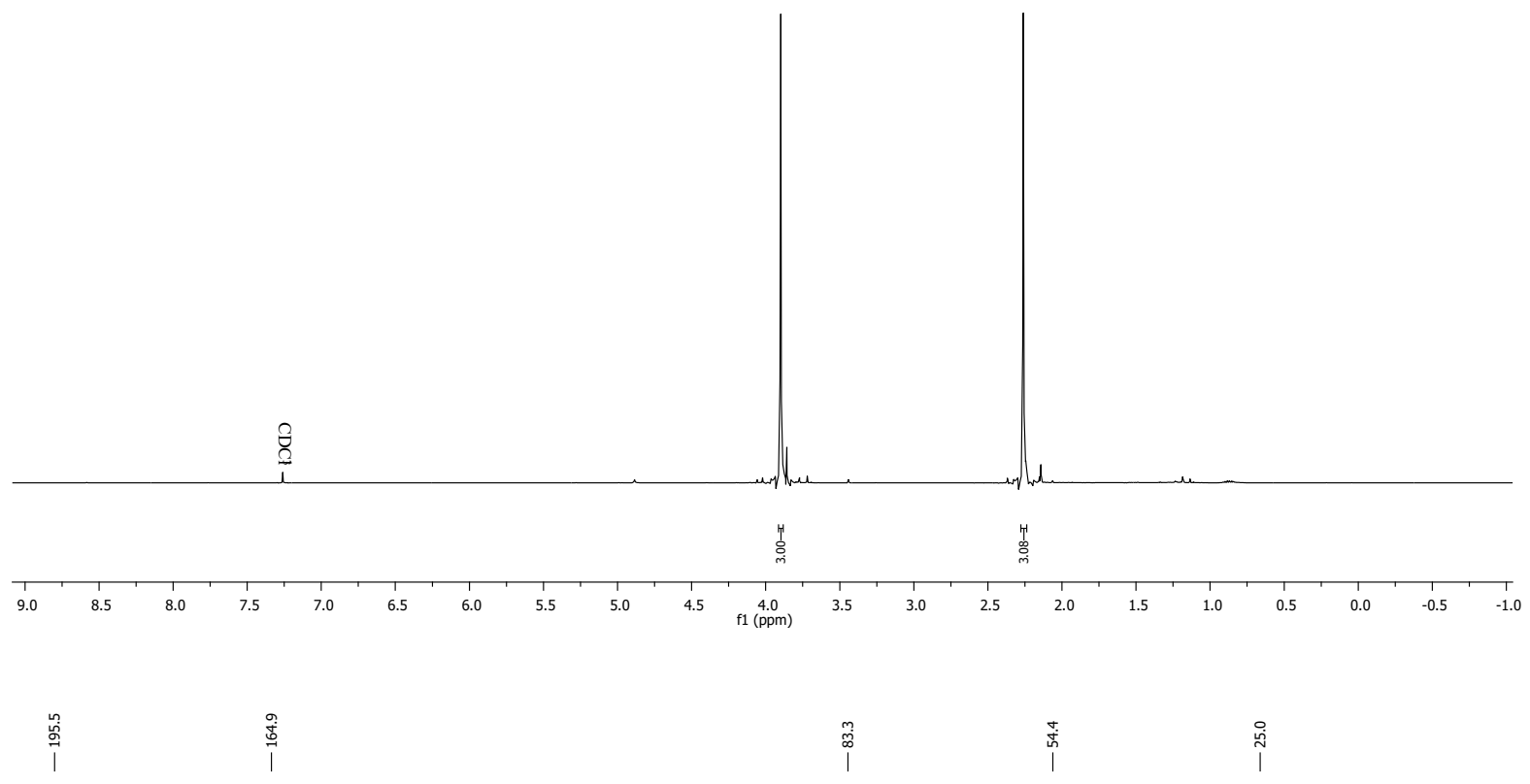

${ }^{13} \mathrm{C} \mathrm{NMR}(151 \mathrm{MHz}, \mathrm{CDCl})$
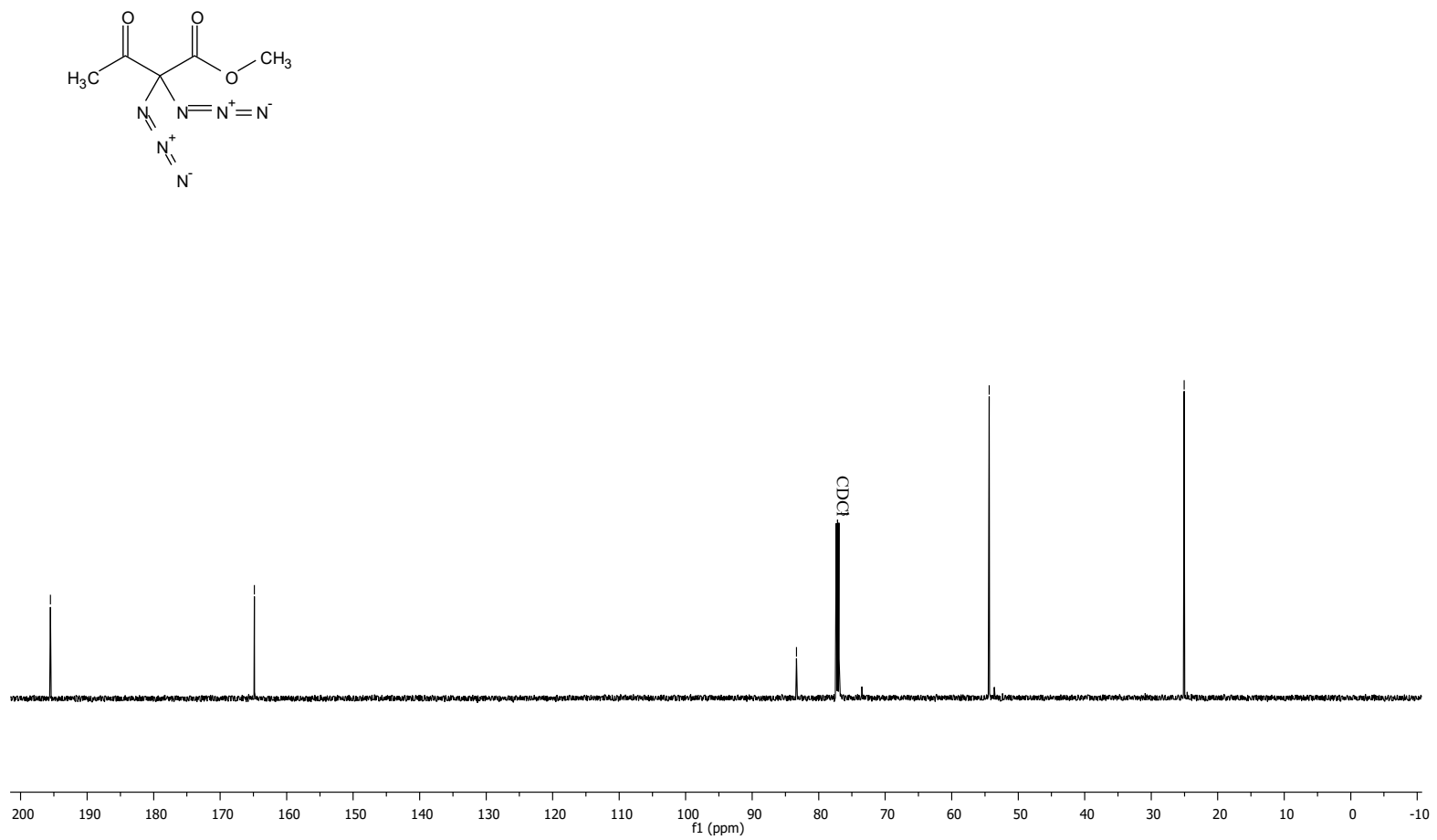

8 


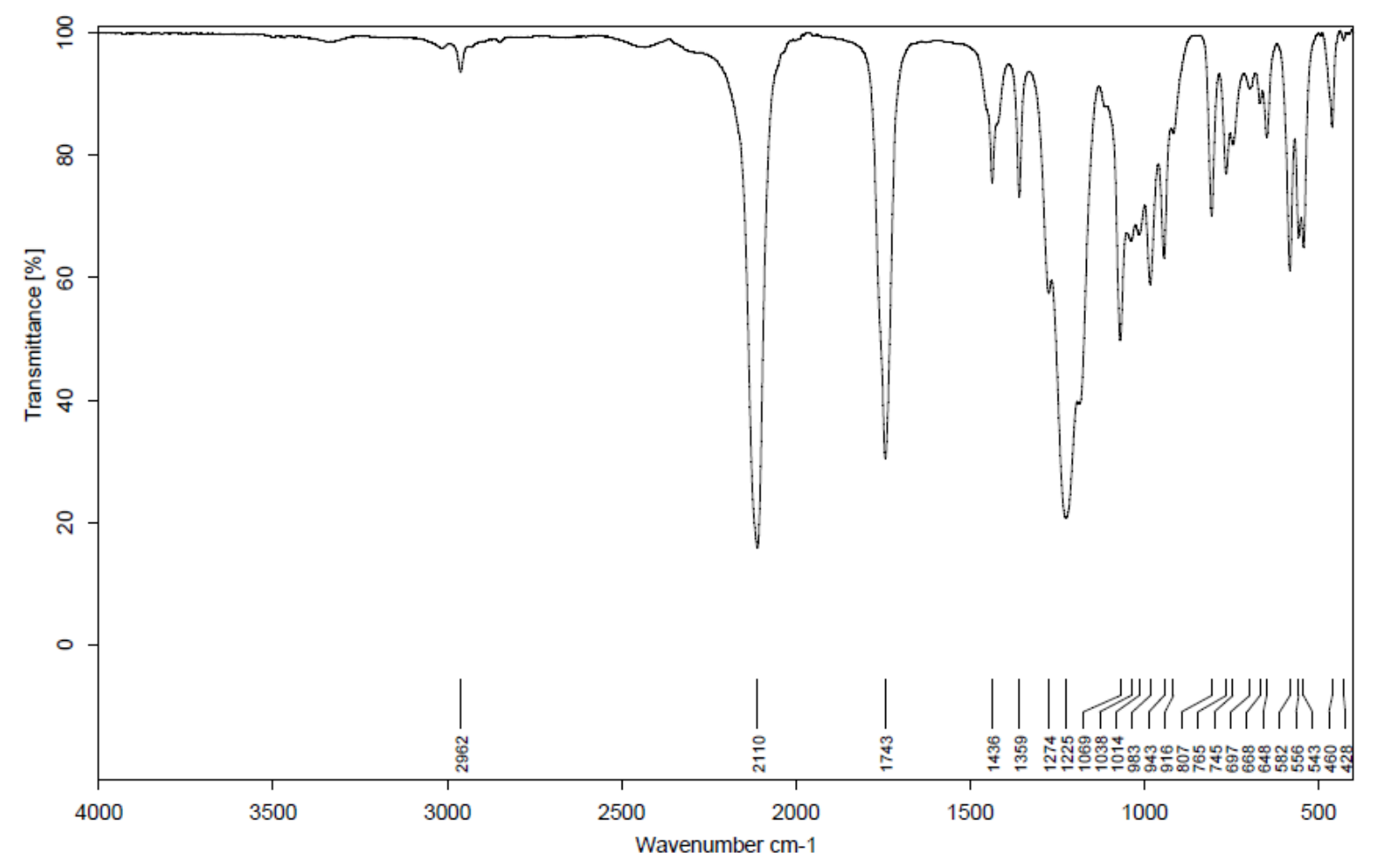

Methyl 2,2-bis(4,5,6,7,8,9-hexahydro-1H-cycloocta[d][1,2,3]triazol-1-yl)-3-oxobutanoate

${ }^{1} \mathrm{H}$ NMR(600MHz, CDCl)
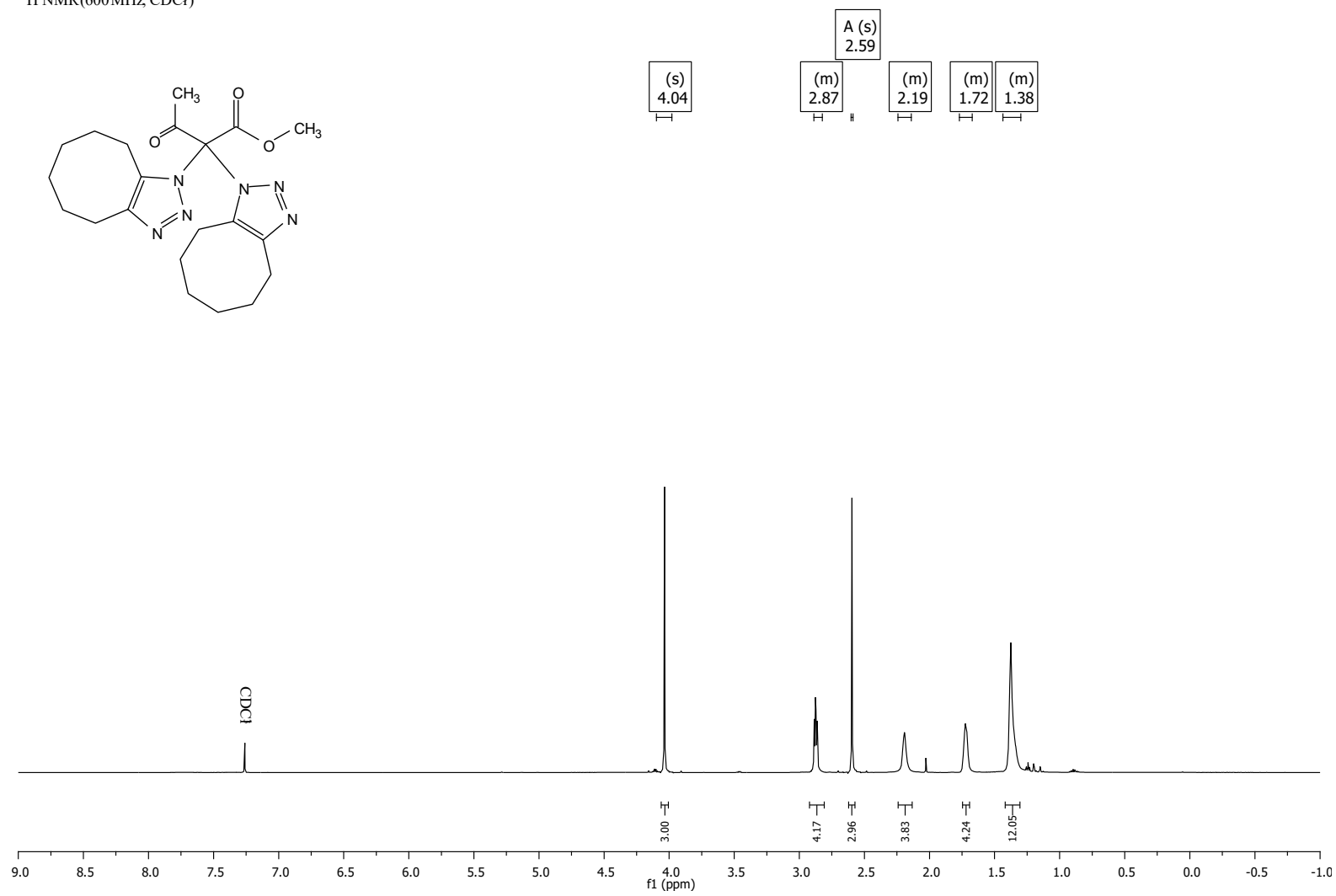


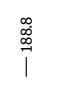

$\stackrel{\text { I }}{\stackrel{0}{0}}$

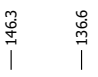

$\underset{\substack{\mathscr{D} \\ 1}}{1}$

诂

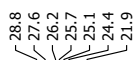

${ }^{13} \mathrm{C} \mathrm{NMR}(151 \mathrm{MHz}, \mathrm{CDCl})$
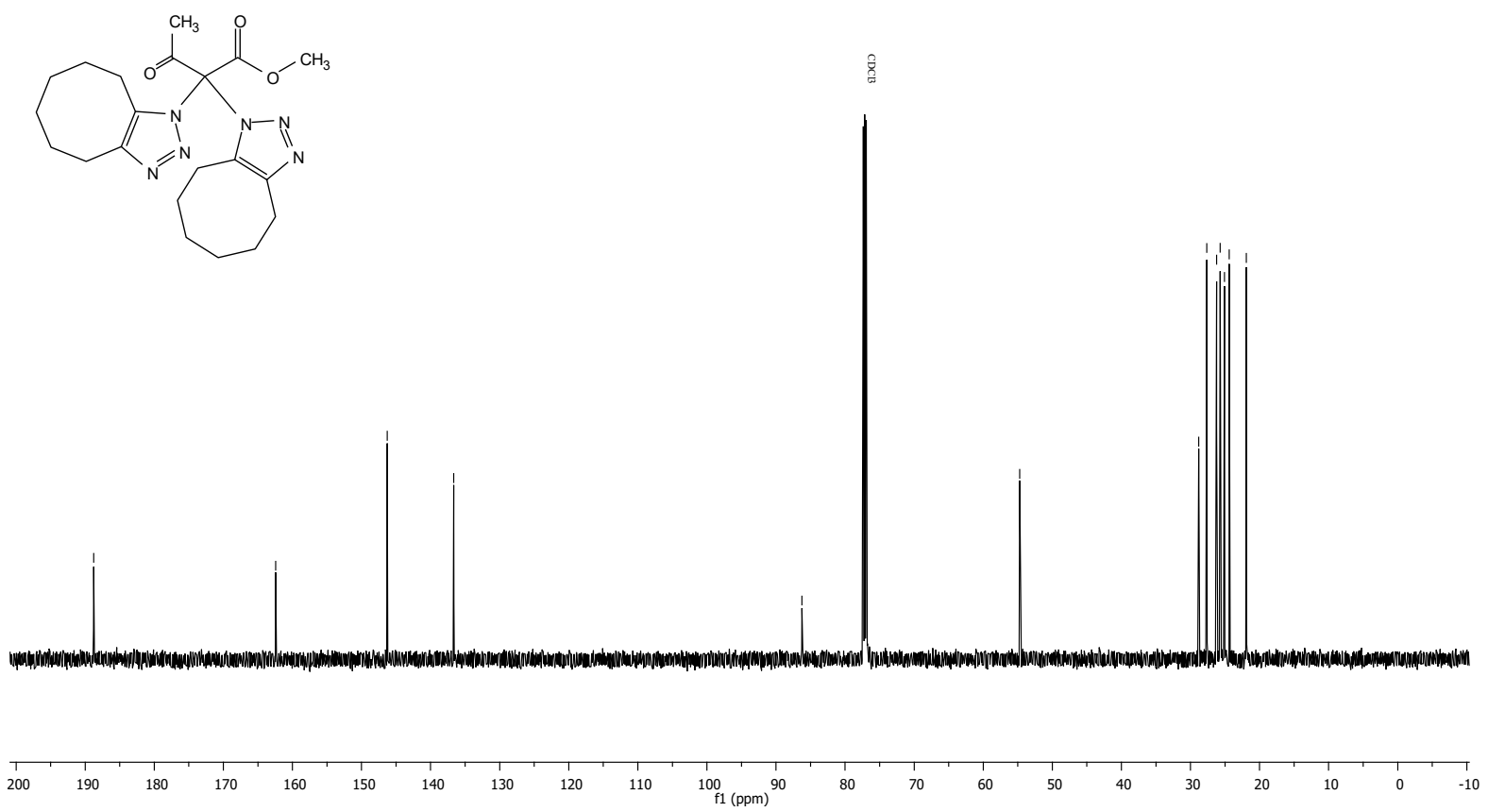

Ethyl 2,2-diazido-3-oxobutanoate (2f)

${ }^{1} \mathrm{H}$ NMR(400MHz, CDCl)

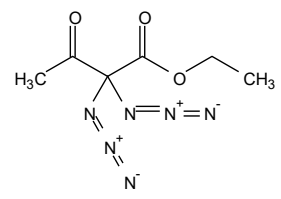

\begin{tabular}{|r|}
\hline$(q)$ \\
4.37 \\
\hline$H$
\end{tabular}
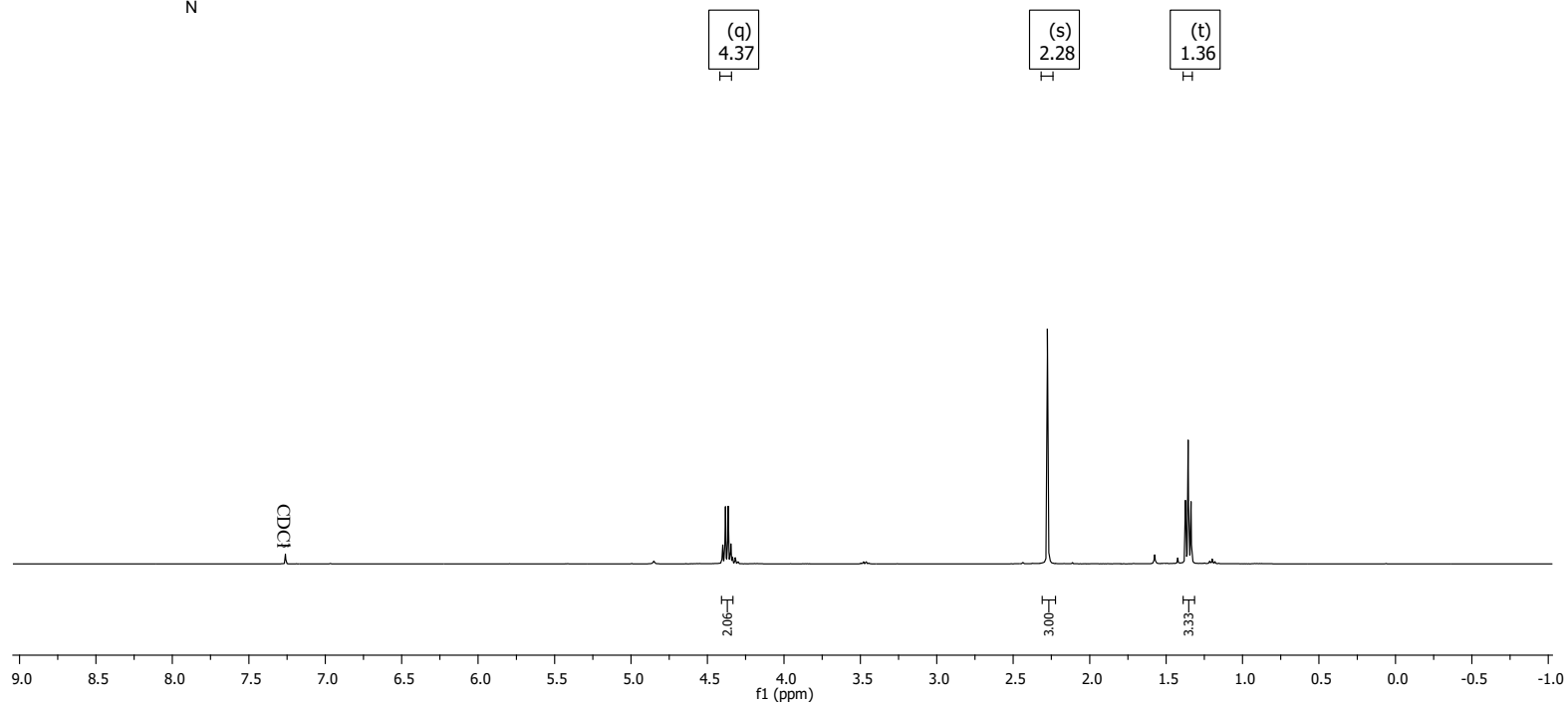

10 


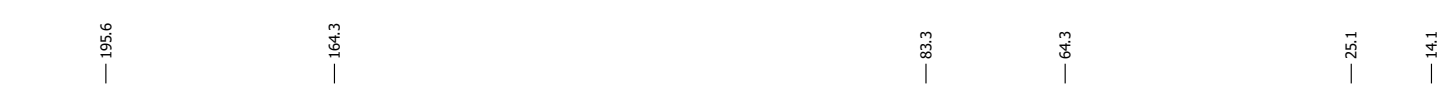

${ }^{13} \mathrm{C}$ NMR(101 MHz, CDCP)
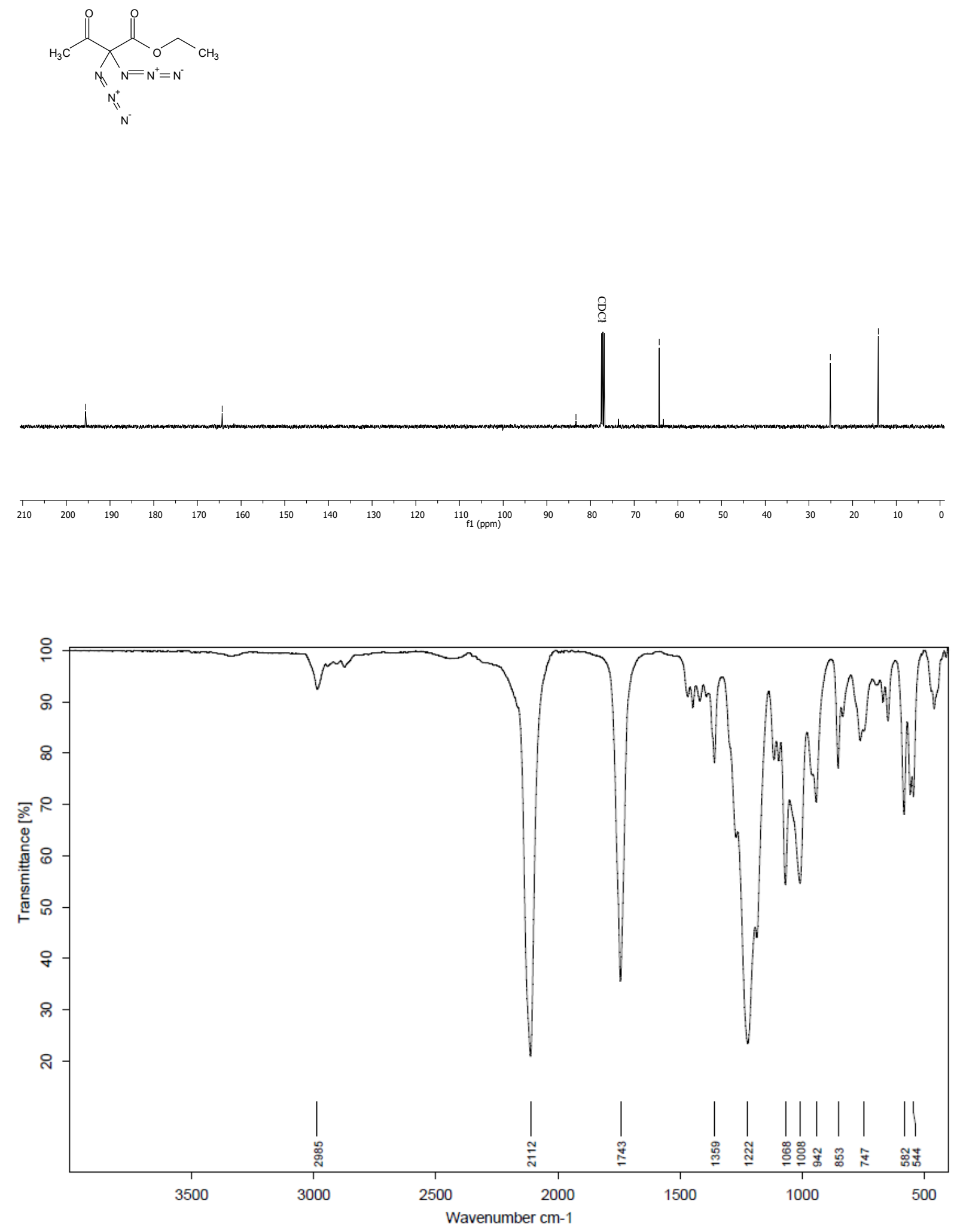
Ethyl 2,2-bis(4,5,6,7,8,9-hexahydro-1H-cycloocta[d][1,2,3]triazol-1-yl)-3-oxobutanoate
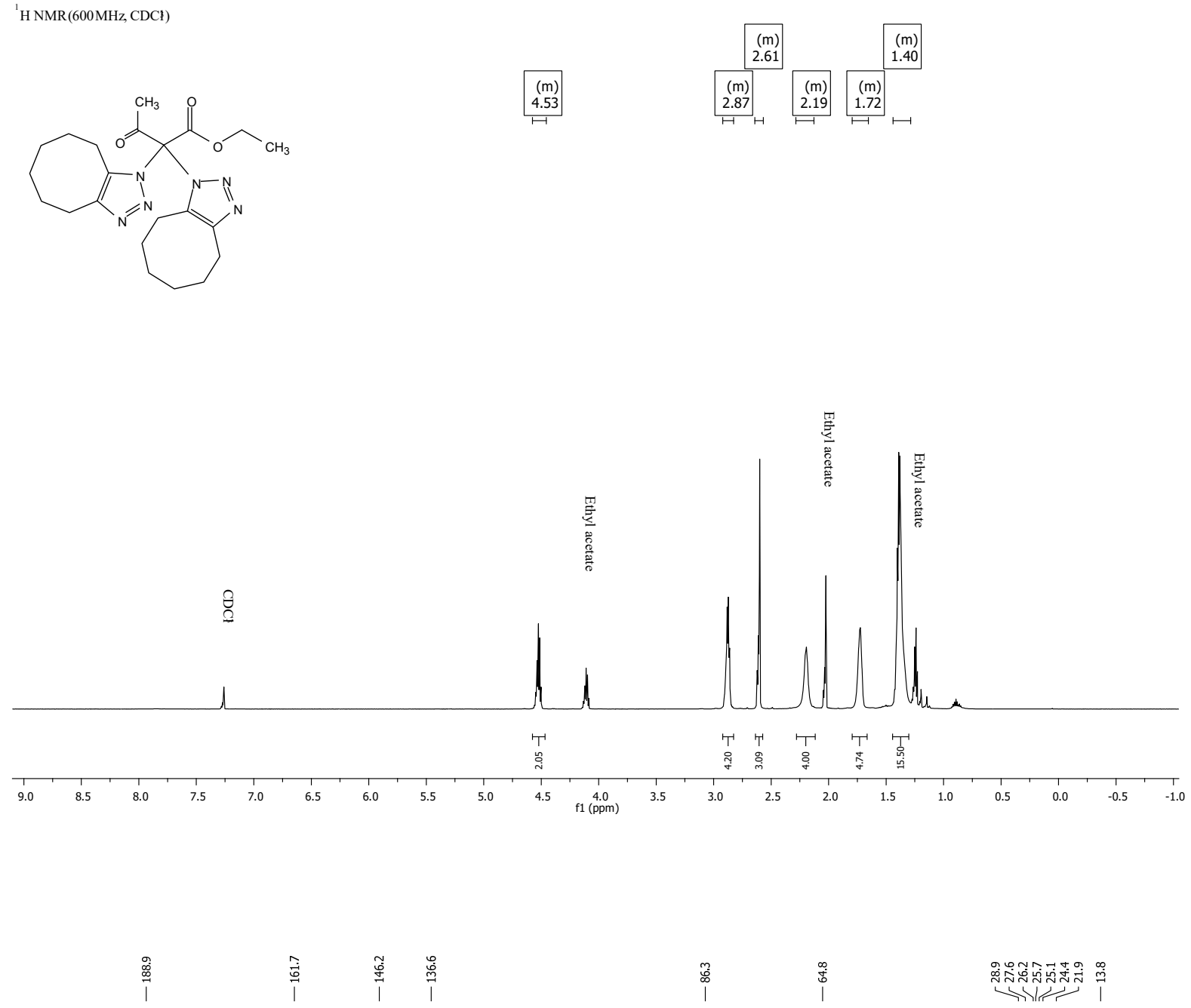

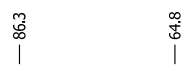

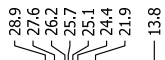

${ }^{13} \mathrm{C}$ NMR $(151 \mathrm{MHz}, \mathrm{CDC})$
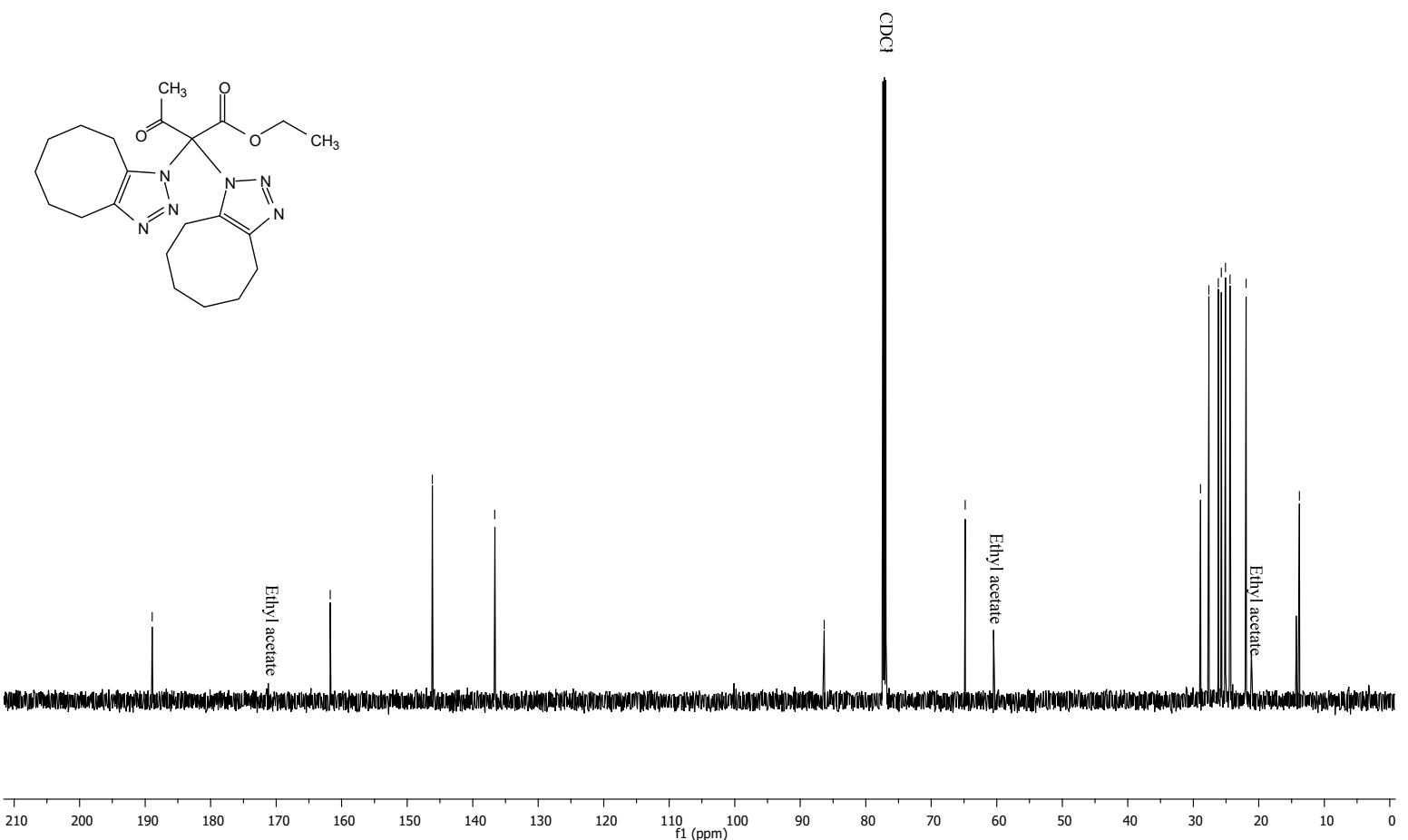
tert-Butyl 2,2-diazido-3-oxobutanoate (2g)

'H NMR(400MHz, CDC1)

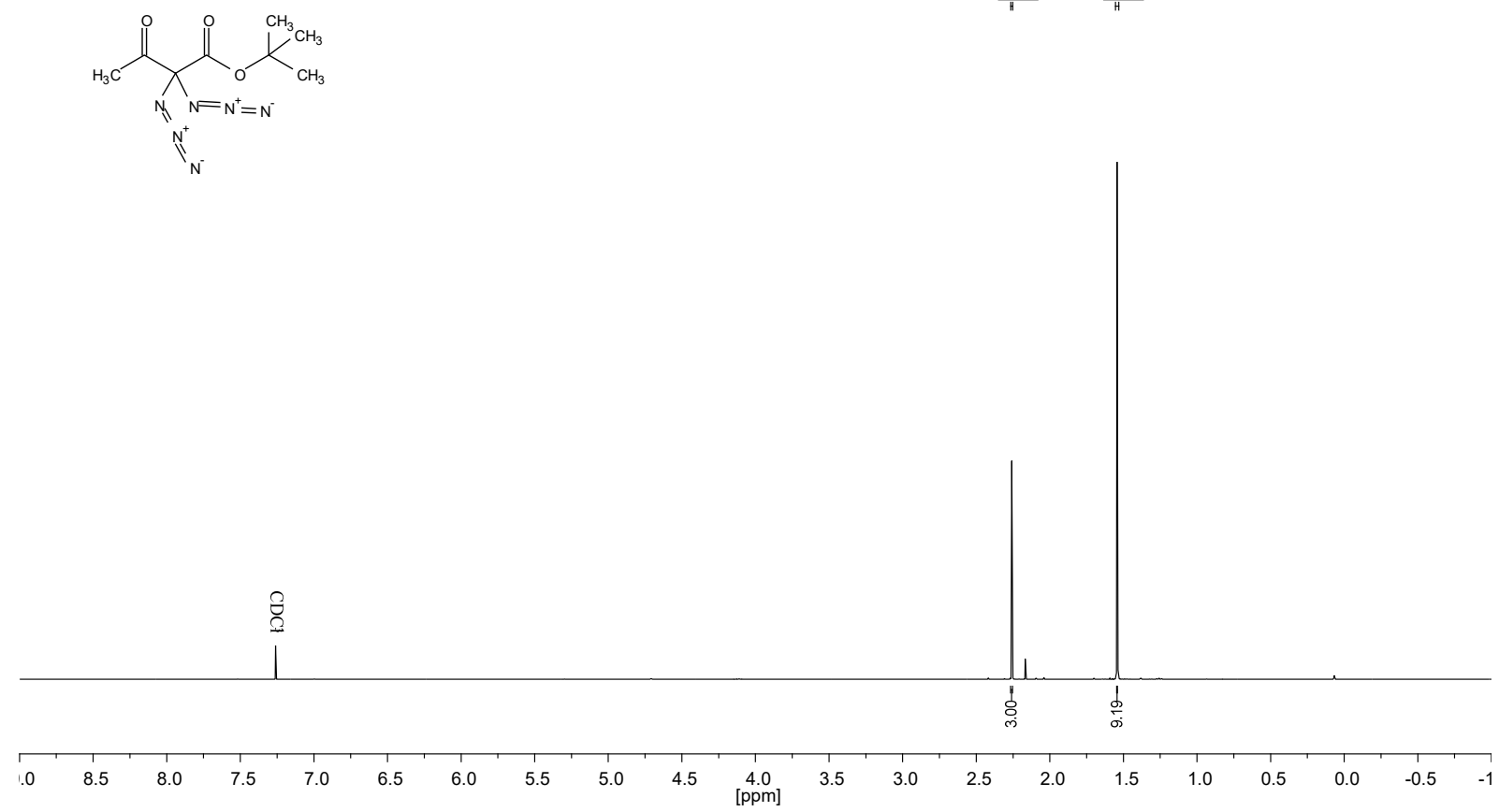

\begin{tabular}{|c|c|}
\hline (s) & (s) \\
2.26 & 1.54 \\
\hline$H$ & H \\
\hline$H$
\end{tabular}

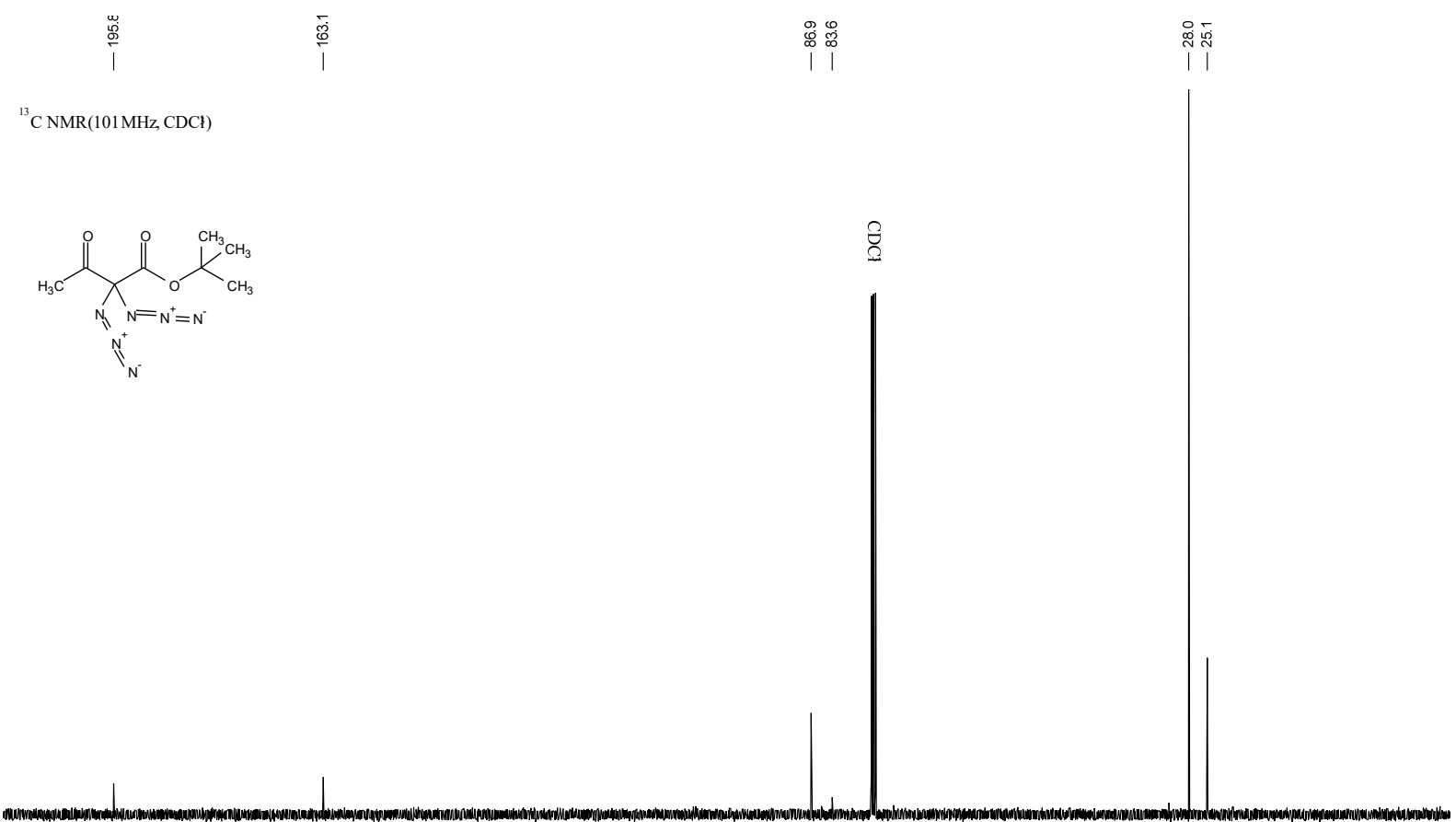

$\begin{array}{llllllllllllllllllllllllllll}210 & 200 & 190 & 180 & 170 & 160 & 150 & 140 & 130 & 120 & 110 & 100 & 90 & 80 & 70 & 60 & 50 & 40 & 30 & 20 & 10 & 0 & -10\end{array}$ 


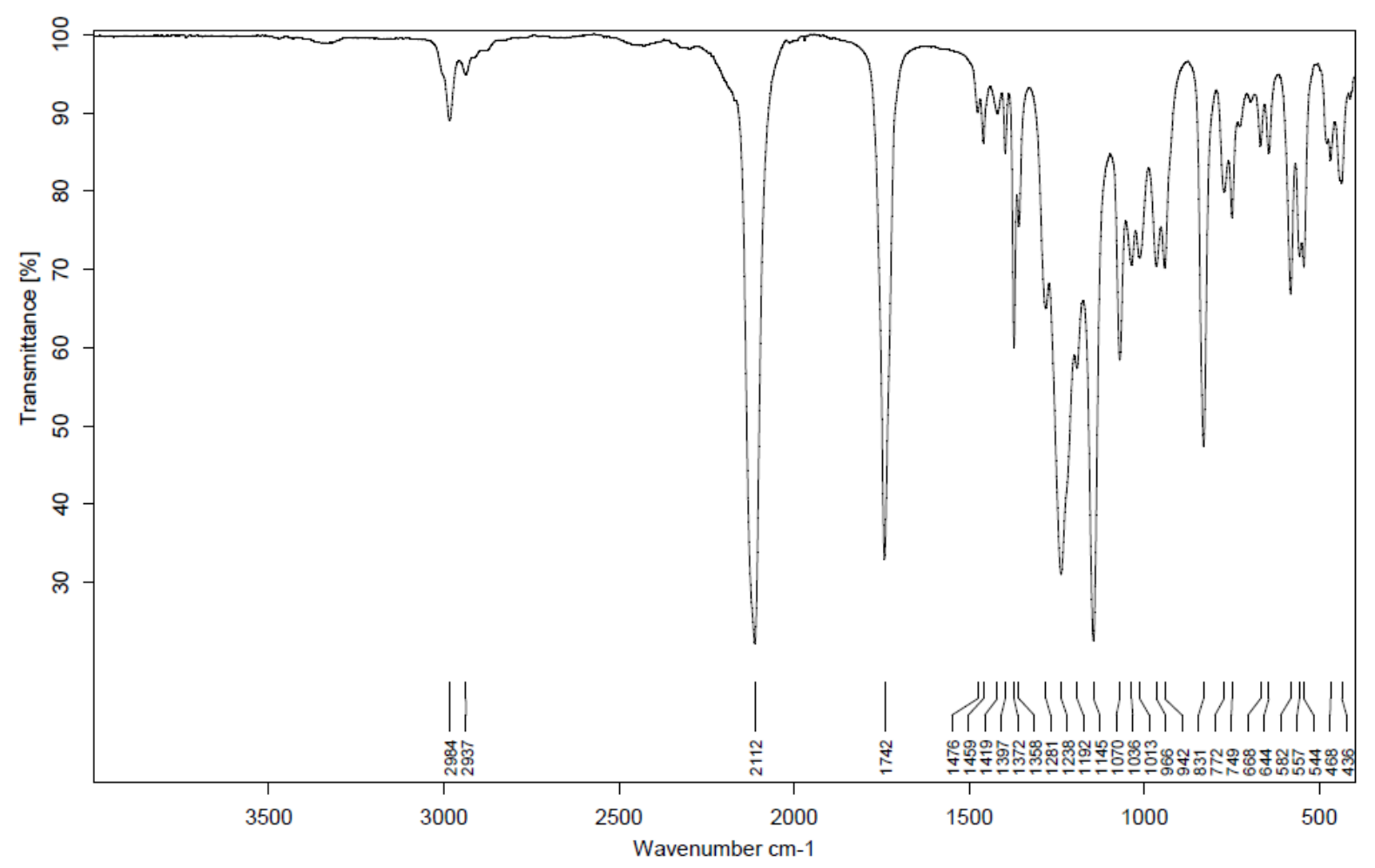

tert-Butyl 3-oxo-2,2-bis(4-phenyl-1H-1,2,3-triazol-1-yl)butanoate

${ }^{1} \mathrm{H} \mathrm{NMR}(600 \mathrm{MHz}, \mathrm{CDCl})$
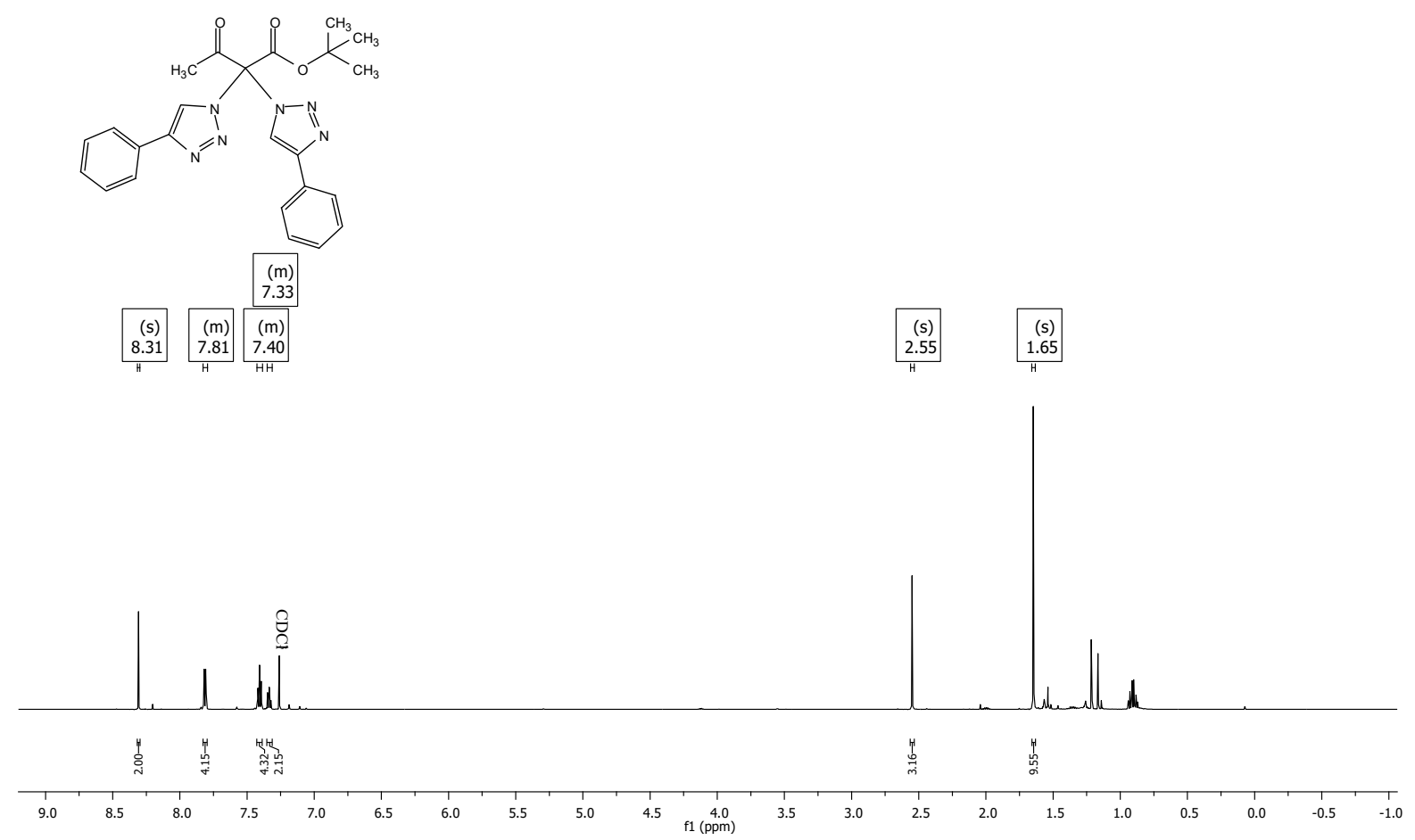

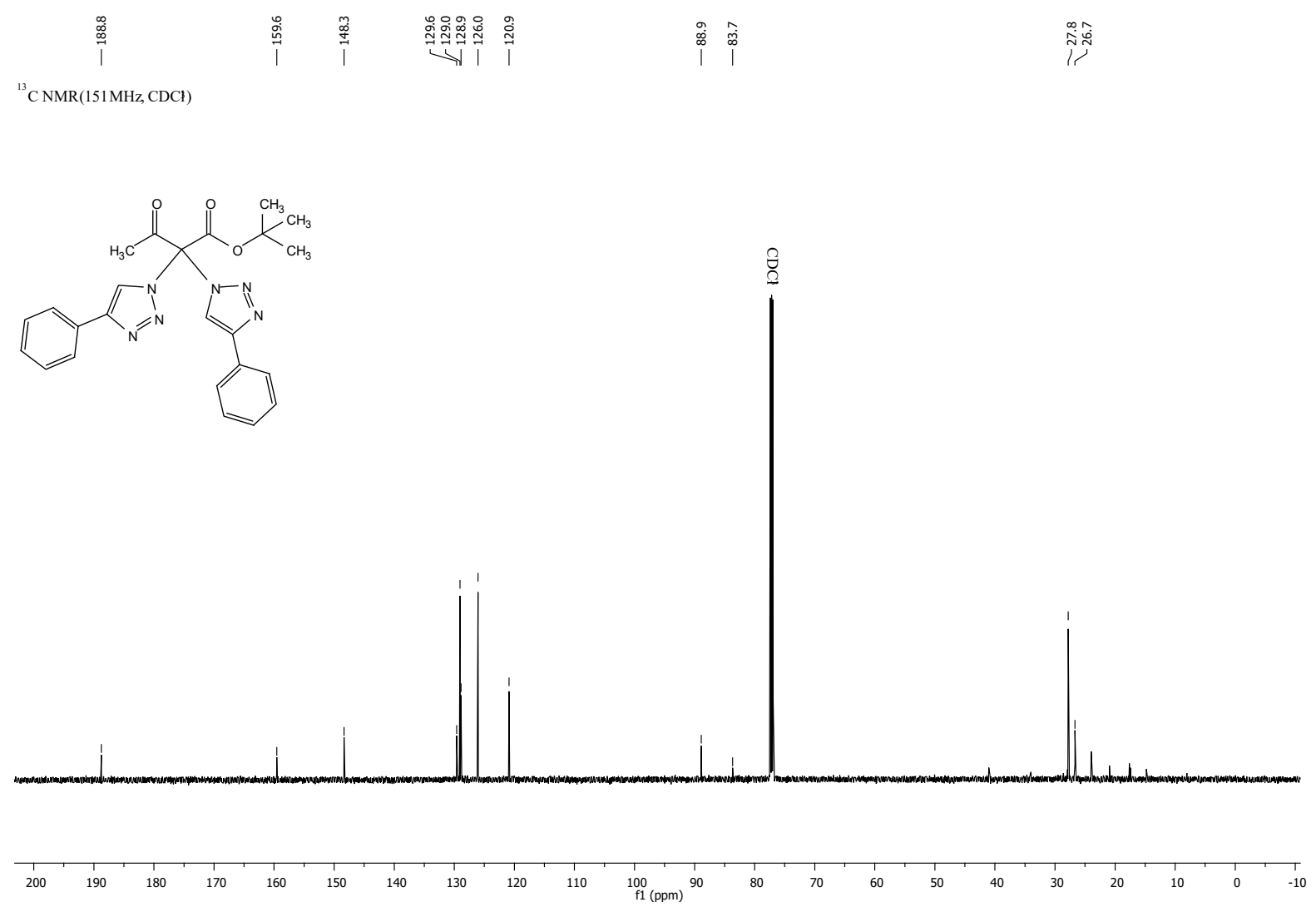

tert-Butyl 2,2-diazido-5-hydroxy-6-methyl-3-oxoheptanoate (2h)

${ }^{1} \mathrm{H} \mathrm{NMR}(400 \mathrm{MHz}, \mathrm{CDCl})$
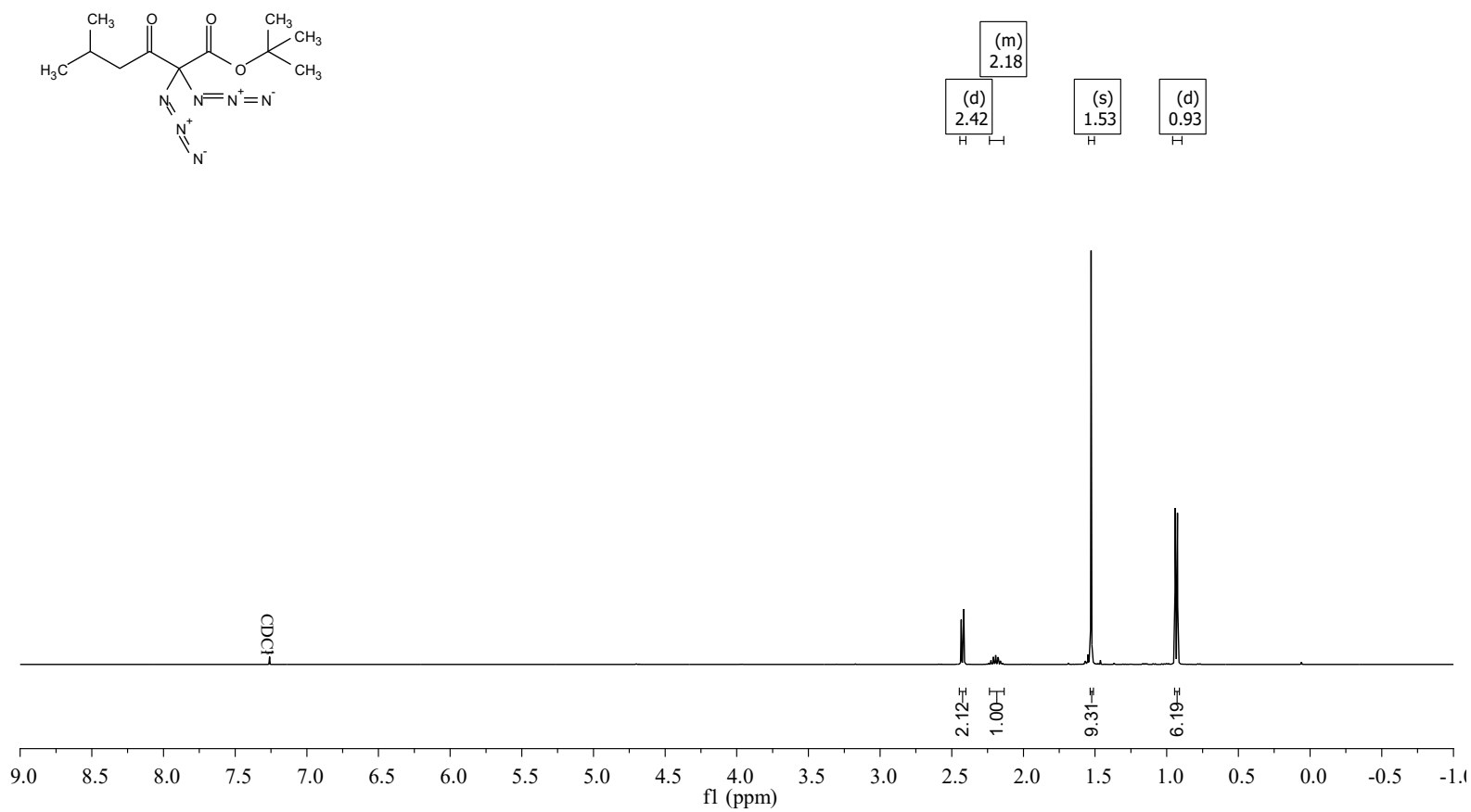


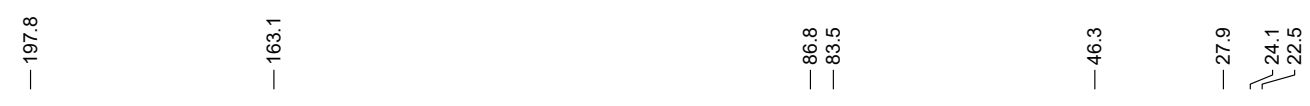

${ }^{13} \mathrm{C} \mathrm{NMR}(101 \mathrm{MHz}, \mathrm{CDCl})$
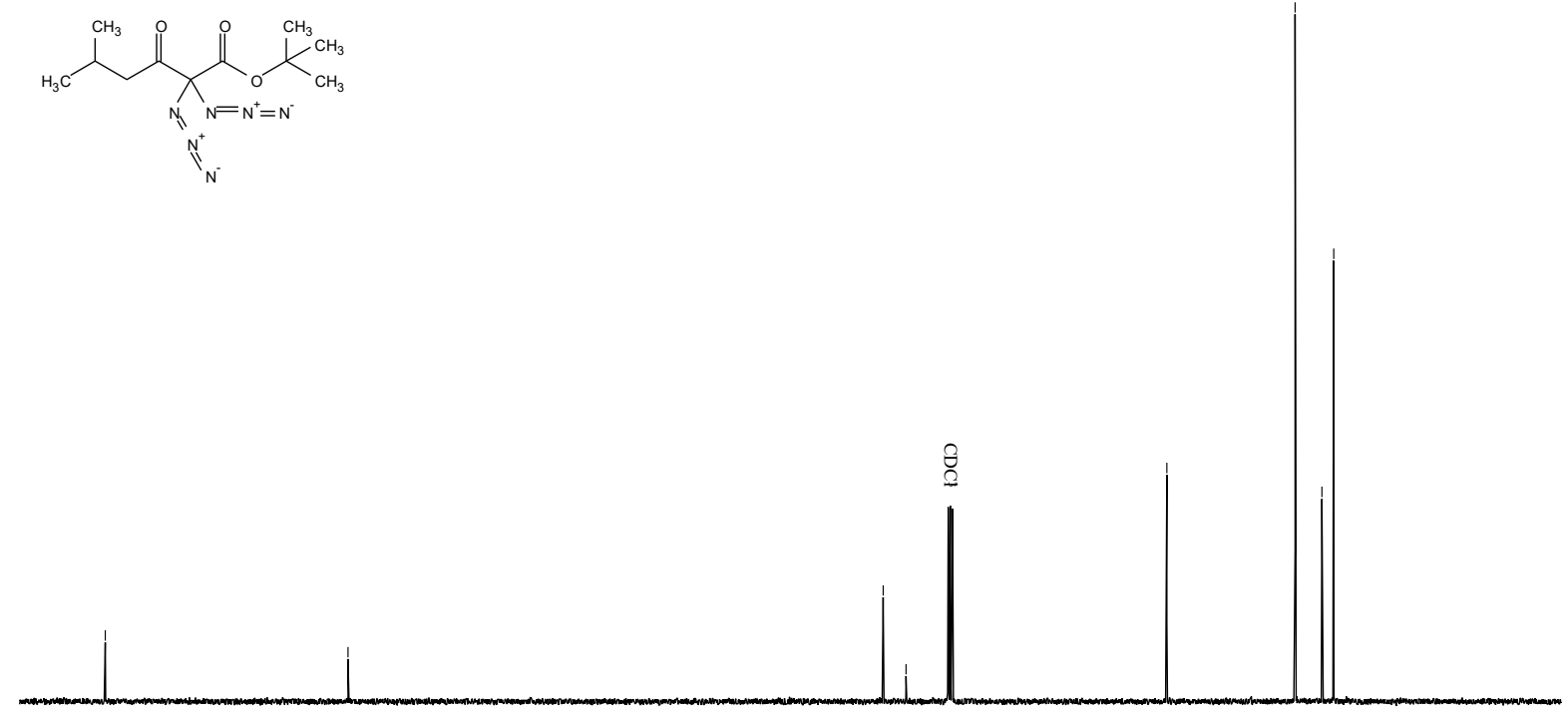

\begin{tabular}{rlllllllllllllllllllllll}
\hline 210 & 200 & 190 & 180 & 170 & 160 & 150 & 140 & 130 & 120 & 110 & 100 & 90 & 80 & 70 & 60 & 50 & 40 & 30 & 20 & 10 & 0 & -1(
\end{tabular}

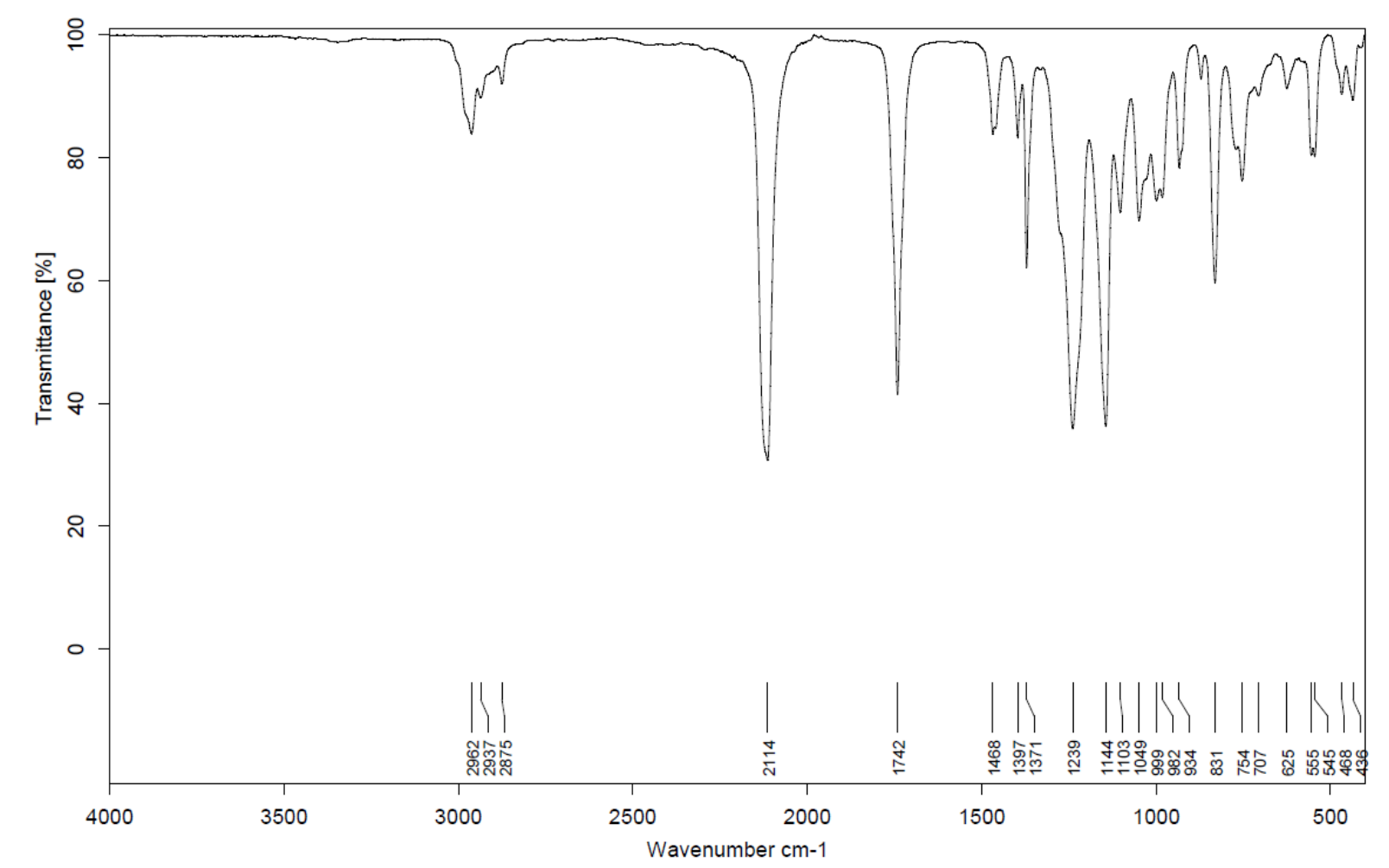


tert-Butyl 2,2-bis(4,5,6,7,8,9-hexahydro-1H-cycloocta[d][1,2,3]triazol-1-yl)-5-methyl-3oxohexanoate

${ }^{1} \mathrm{H}$ NMR(400MHz, CDCl)
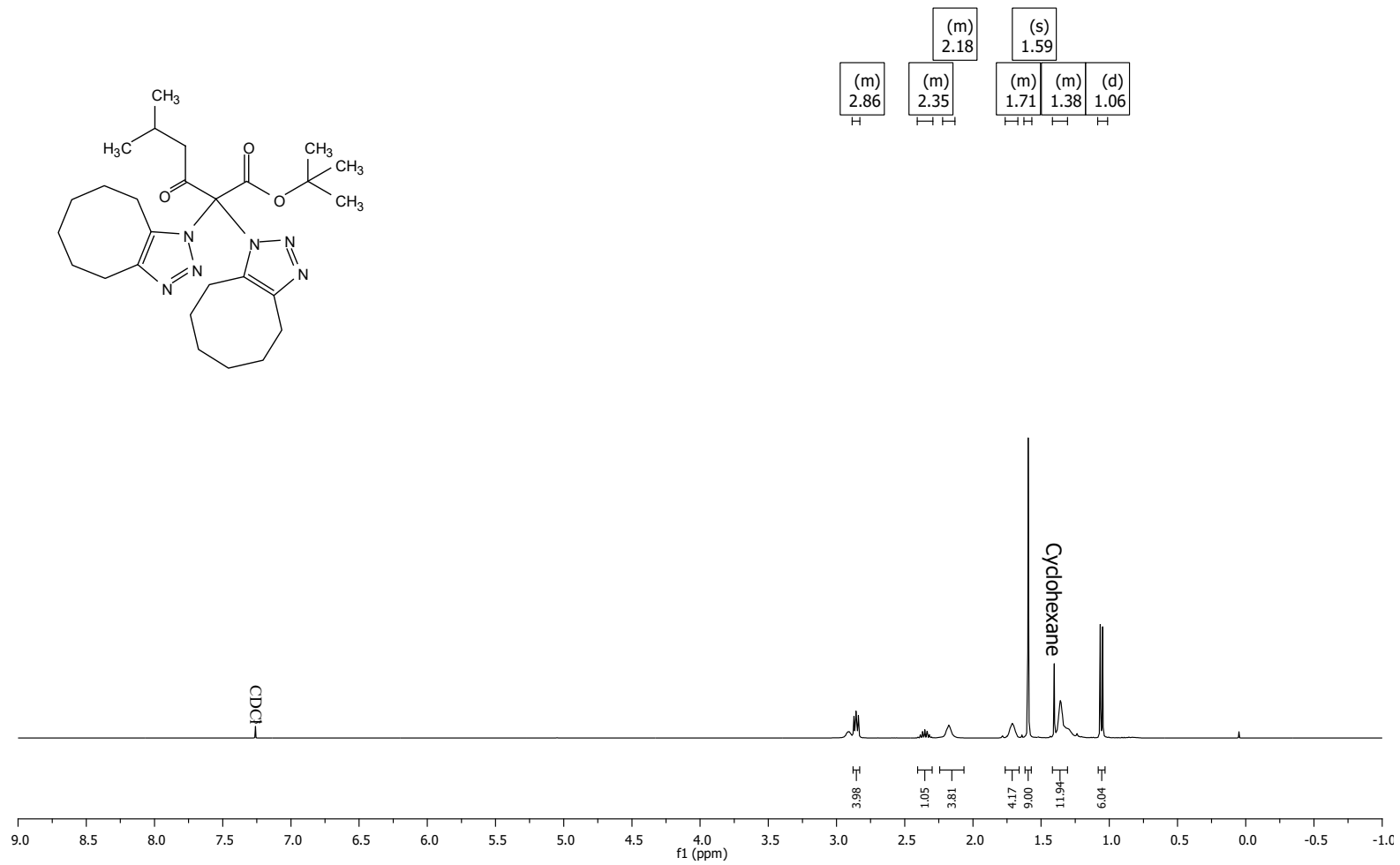

إj

${ }^{13} \mathrm{CNMR}(101 \mathrm{MHz}, \mathrm{CDCl})$

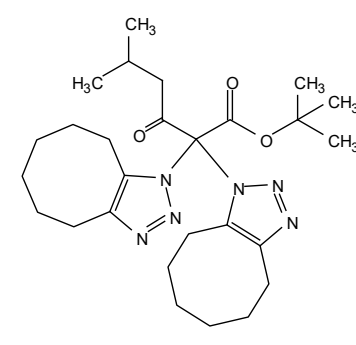

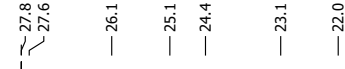
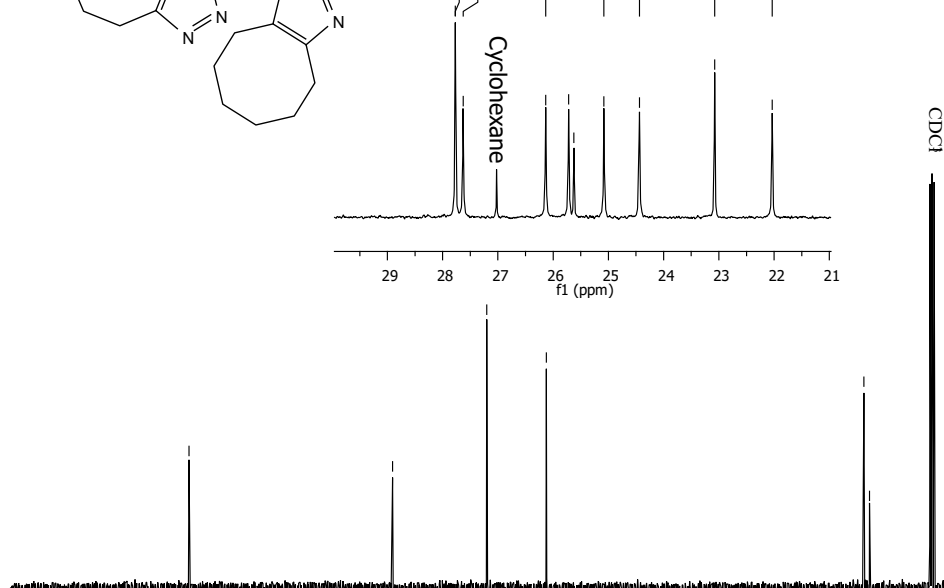

\&

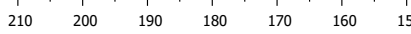

$10 \begin{gathered}100 \\ \mathrm{f} 1(\mathrm{ppm})\end{gathered} 90$ 
tert-Butyl 2,2-diazido-3-oxo-5-phenylpentanoate (2i)

'H NMR(600MHz, CDCl)

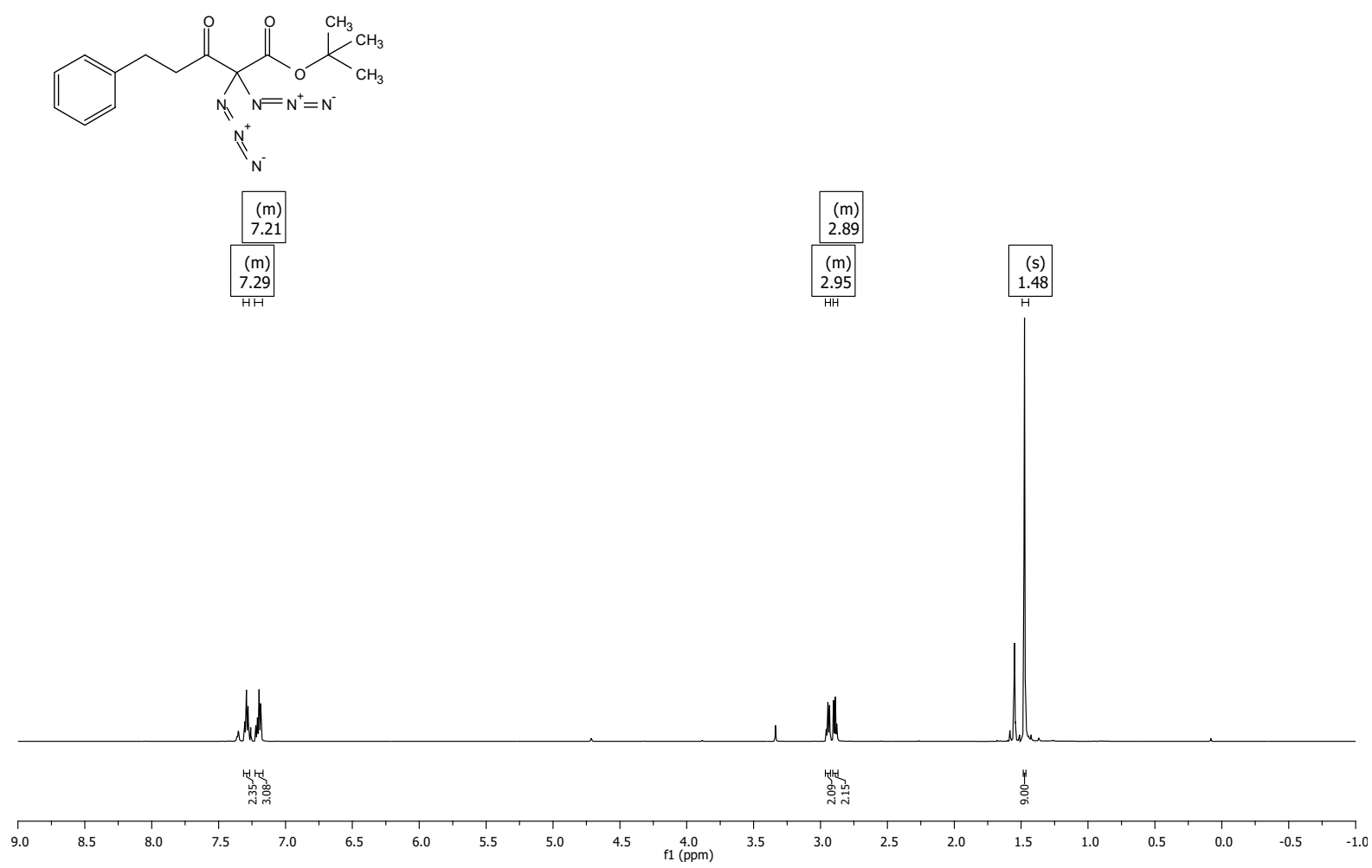

\begin{tabular}{|c|c|c|c|c|c|}
\hline$\stackrel{\stackrel{0}{0}}{\stackrel{d}{i}}$ & إْ & 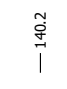 & 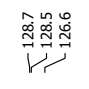 & 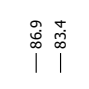 & $\begin{array}{l}\text { 恋 } \\
i\end{array}$ \\
\hline
\end{tabular}<smiles>CC(C)(C)OC(=O)C(N=N)(C(=O)CCc1ccccc1)C(=O)N=N</smiles>
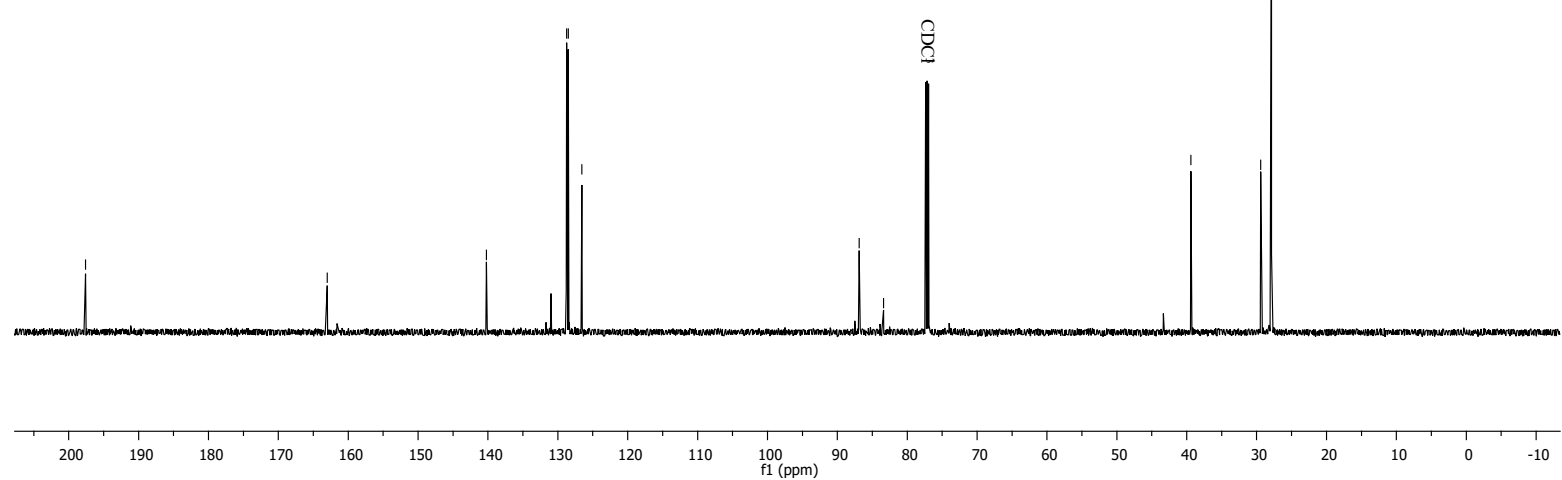


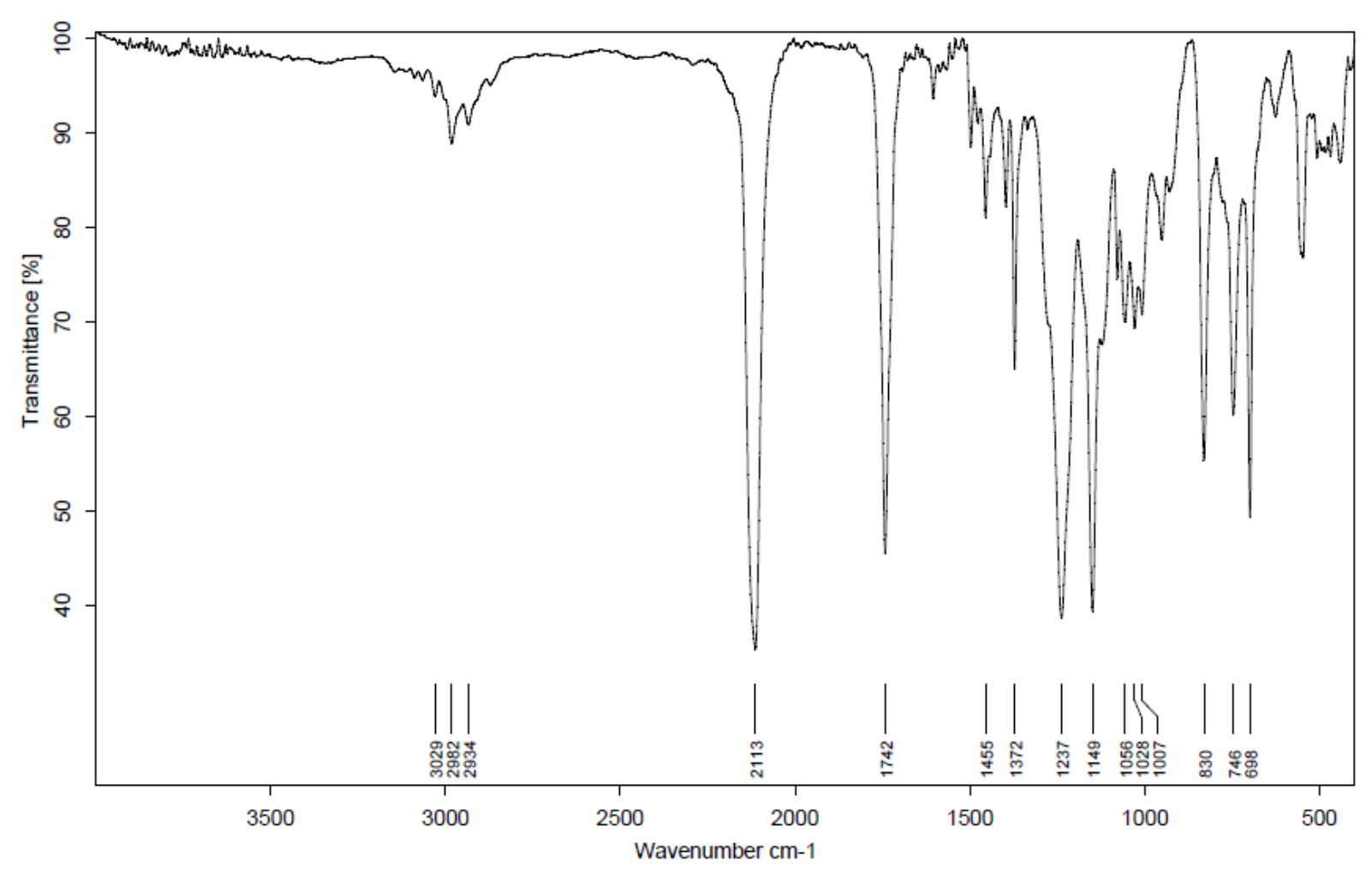

tert-Butyl 2,2-bis(4,5,6,7,8,9-hexahydro-1H-cycloocta[d][1,2,3]triazol-1-yl)-3-oxo-5-phenylpentanoate 'H NMR(400MHz, CDCl)

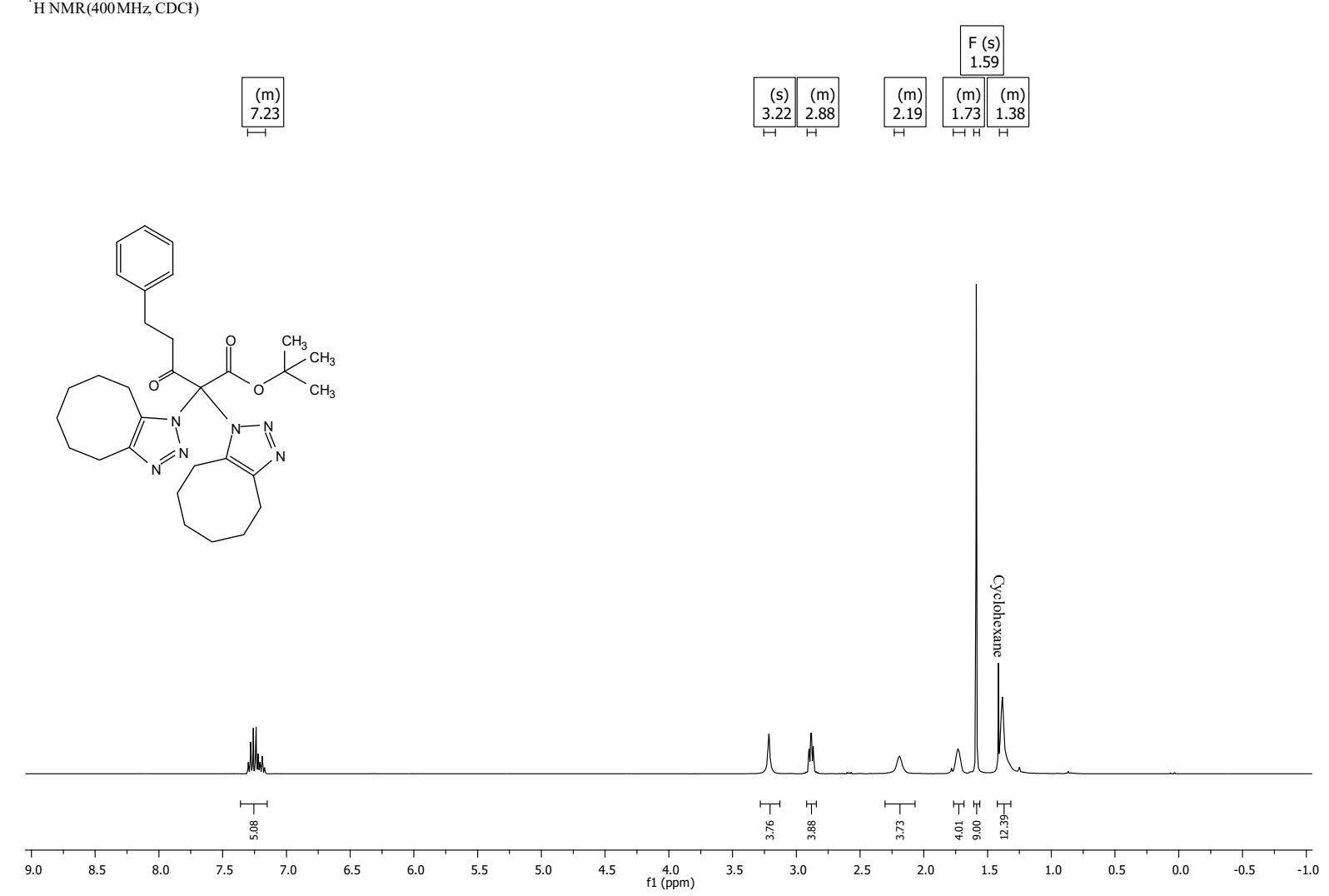




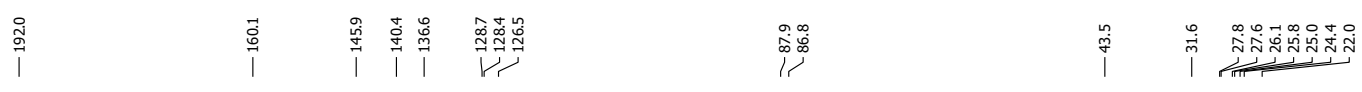

${ }^{13} \mathrm{C} \mathrm{NMR}(101 \mathrm{MHz}, \mathrm{CDCl})$

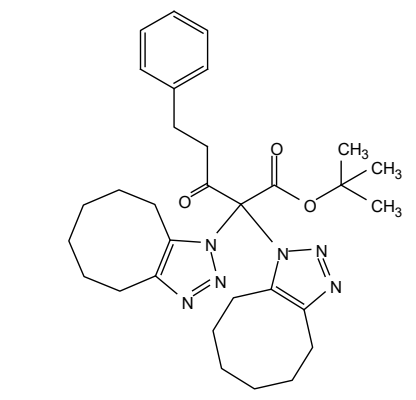

200

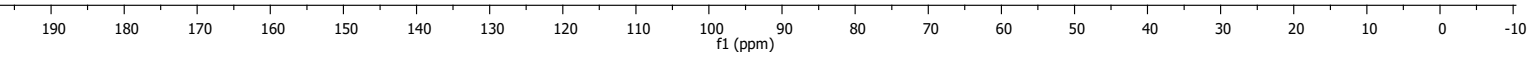

tert-Butyl 2,2-diazido-3-cyclohexyl-3-oxopropanoate (2j)

${ }^{1} \mathrm{H}$ NMR $\left(400 \mathrm{MHz}, \mathrm{C}^{6} \mathrm{D}^{\natural}\right)$
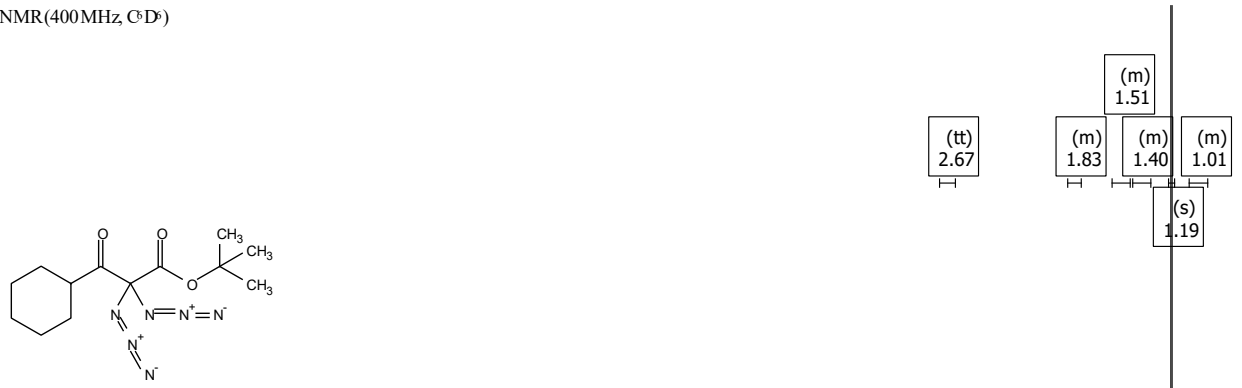

\begin{tabular}{llll}
2.67 & $(\mathrm{~m})$ & $(\mathrm{m})$ & $(\mathrm{m})$ \\
1.83 & 1.40 & 1.01 \\
\hline$\mapsto$ & $\mapsto$ &
\end{tabular}
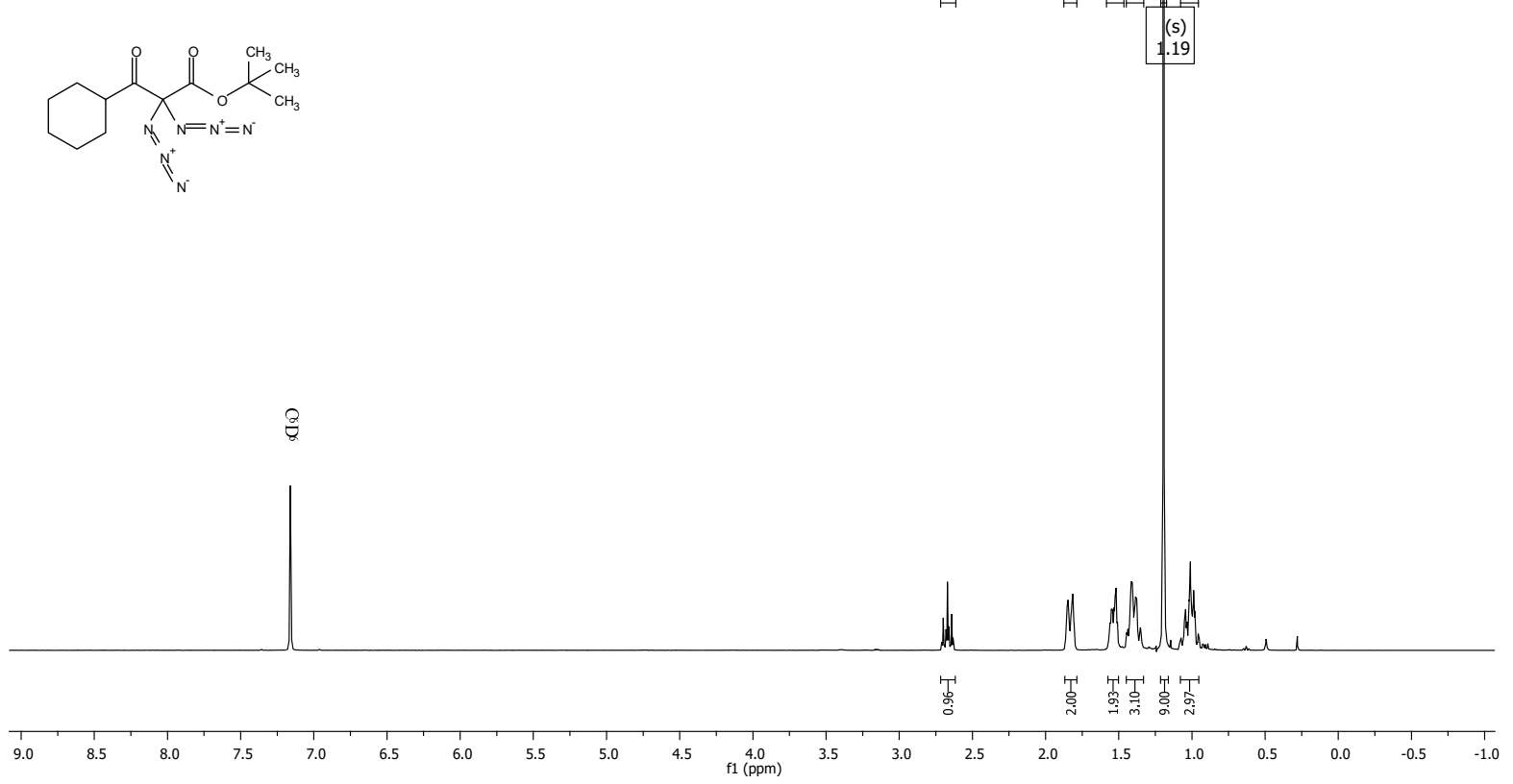

iี

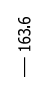
$\prod_{\substack{\infty \\ \infty}}^{\substack{\infty \\ \infty}}$

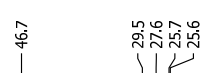

${ }^{13} \mathrm{C} \mathrm{NMR}(101 \mathrm{MHZ}, \mathrm{OD}$ )
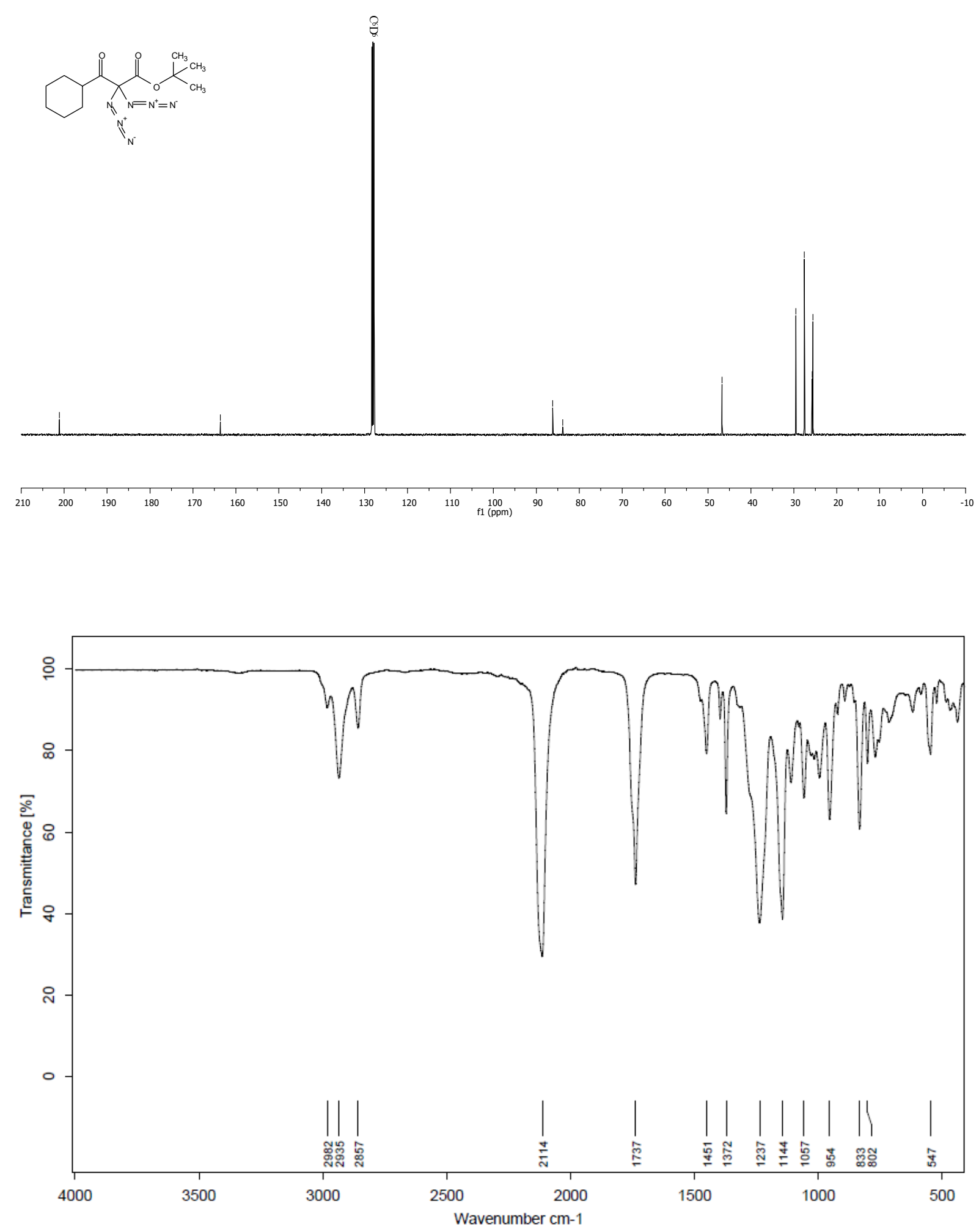
tert-Butyl 3-cyclohexyl-2,2-bis(4,5,6,7,8,9-hexahydro-1H-cycloocta[d] [1,2,3]triazol-1-yl)-3oxopropanoate
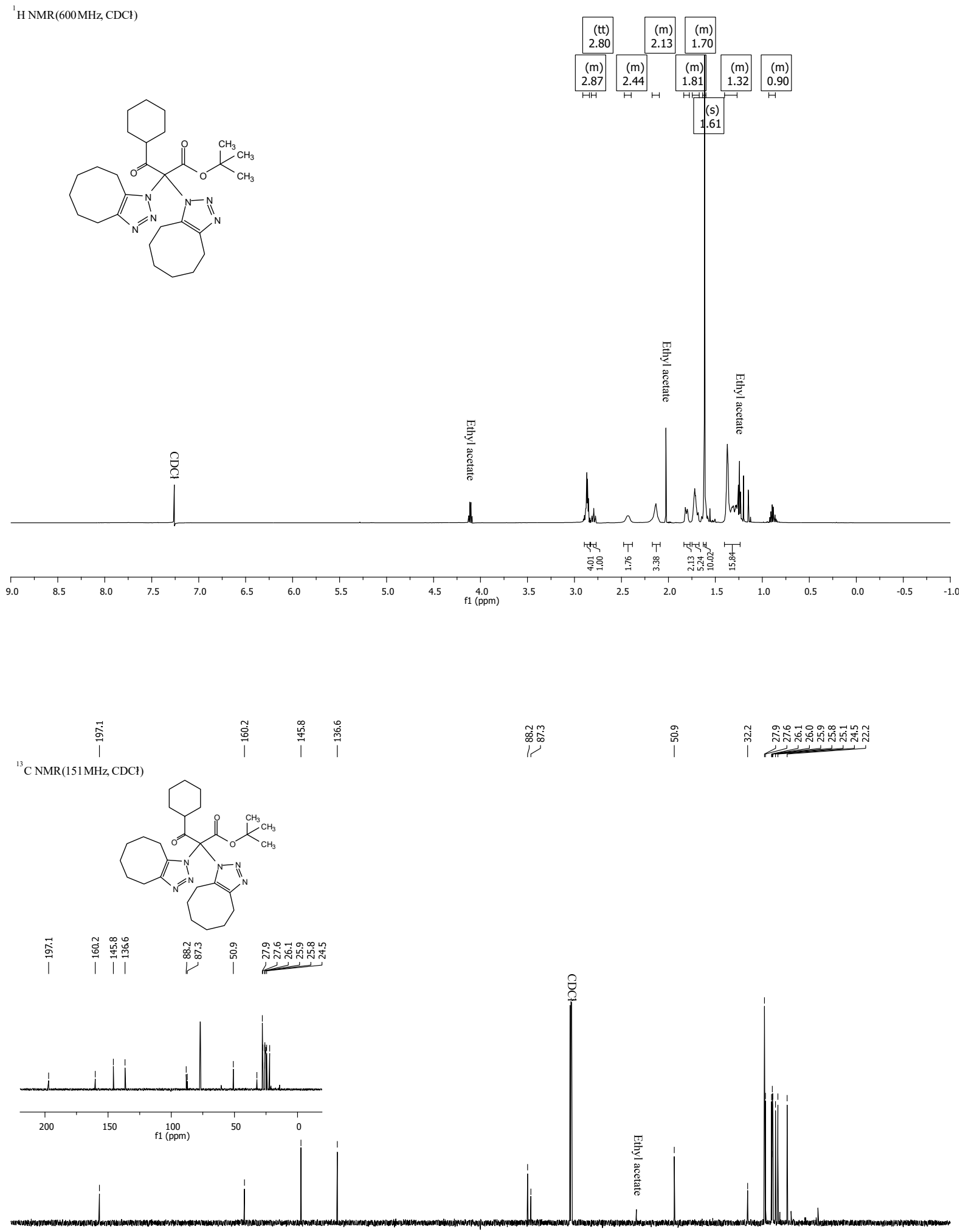
tert-Butyl 2,2-diazido-4,4-dimethyl-3-oxopentanoate (2k)

'H NMR(600MHz, CDCl)
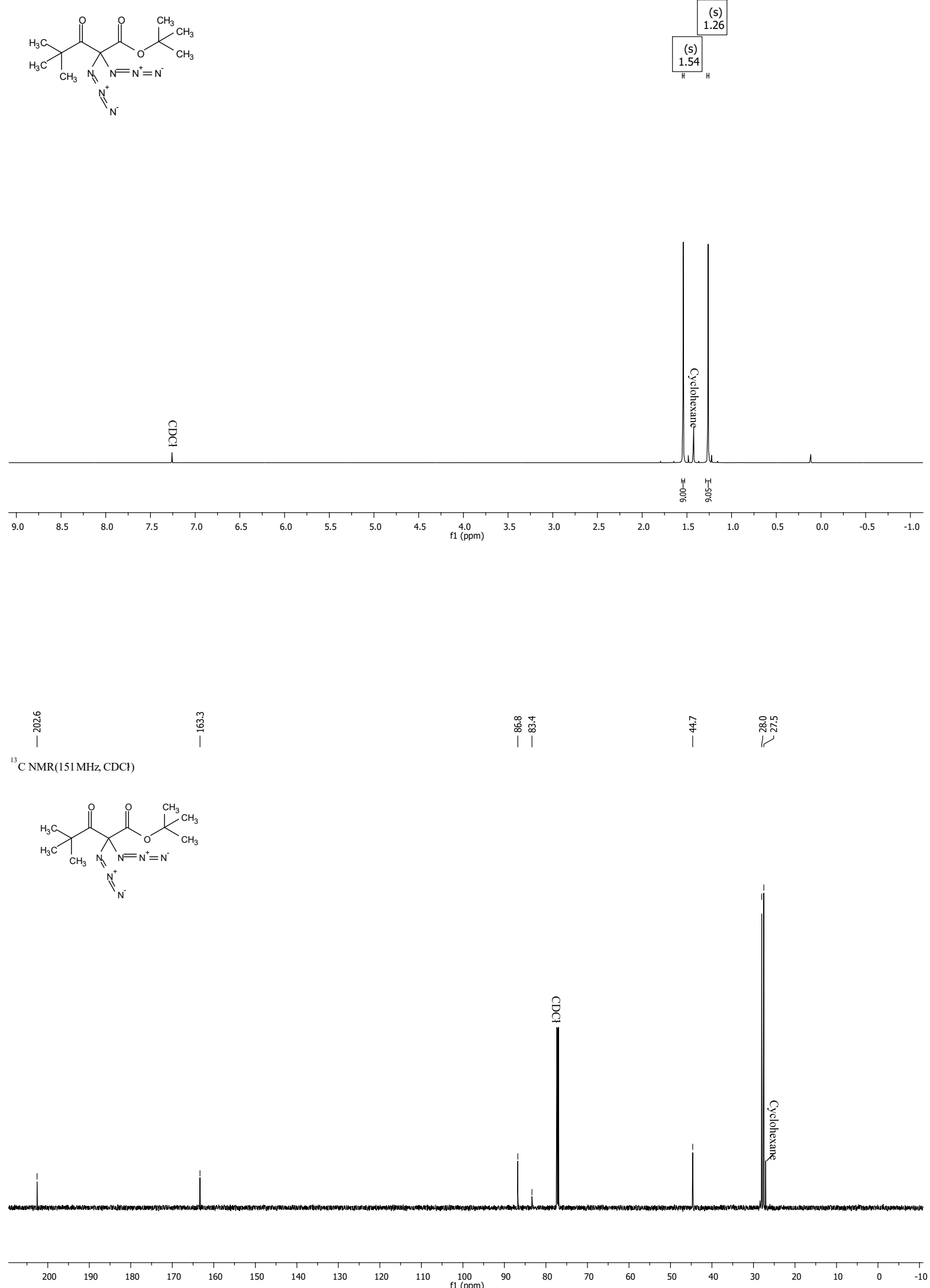


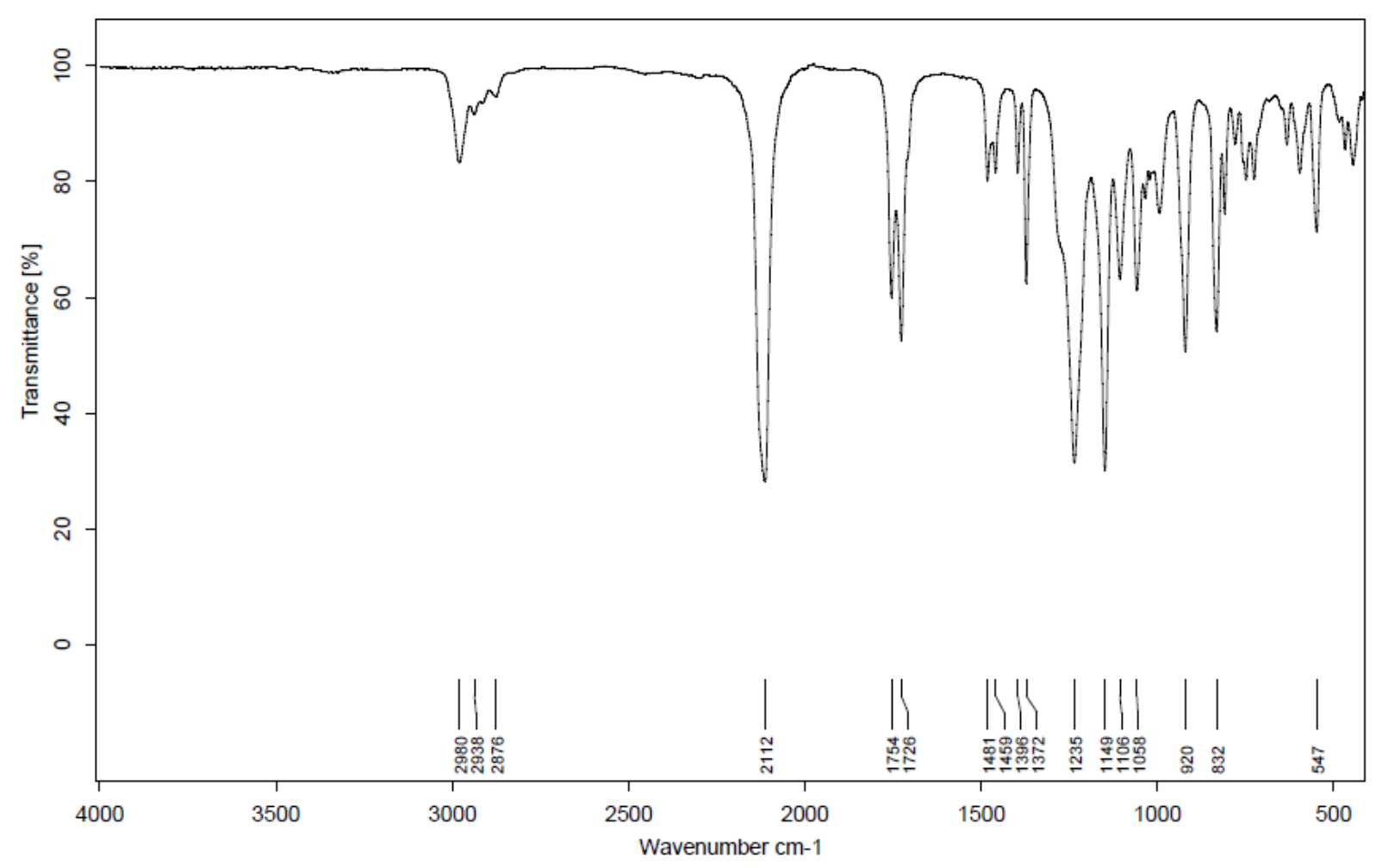

tert-Butyl 2,2-bis(4,5,6,7,8,9-hexahydro-1H-cycloocta[d] [1,2,3]triazol-1-yl)-4,4-dimethyl-3oxopentanoate

${ }^{1} \mathrm{H}$ NMR(400MHz, DMSO)
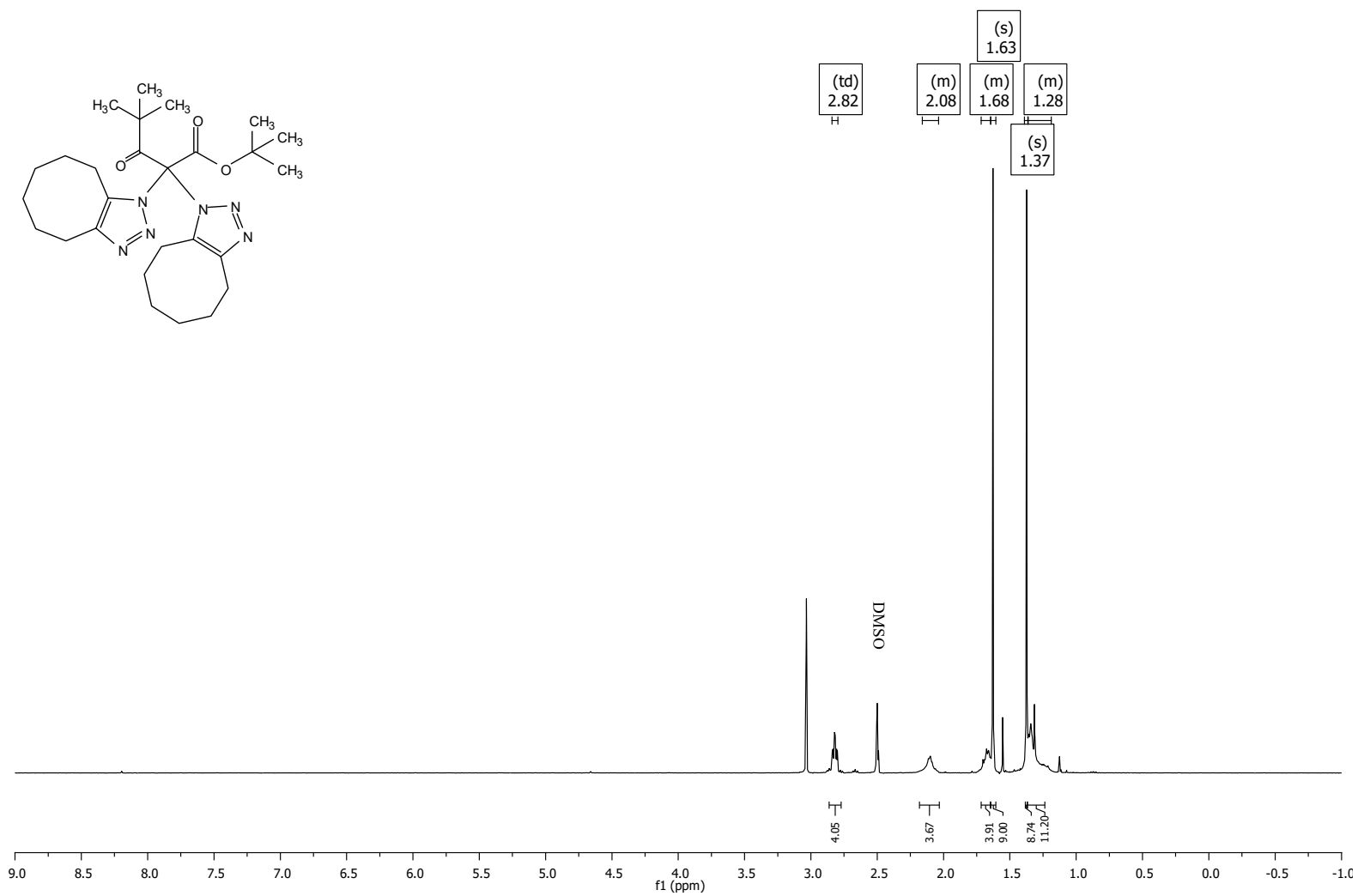

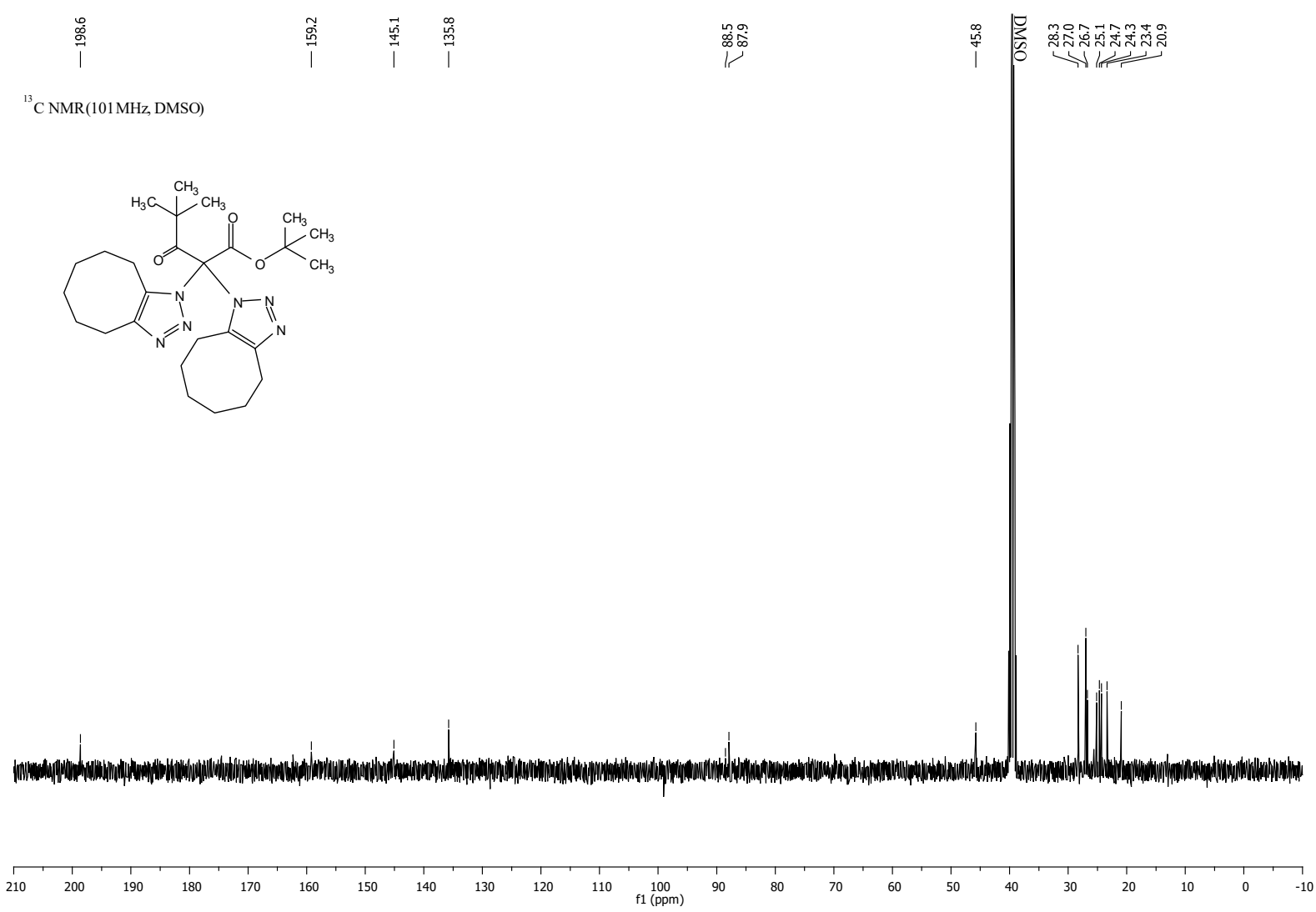

tert-Butyl 3-(adamantan-1-yl)-2,2-diazido-3-oxopropanoate (2l)

${ }^{1} \mathrm{H}$ NMR (400MHz, CDCl)
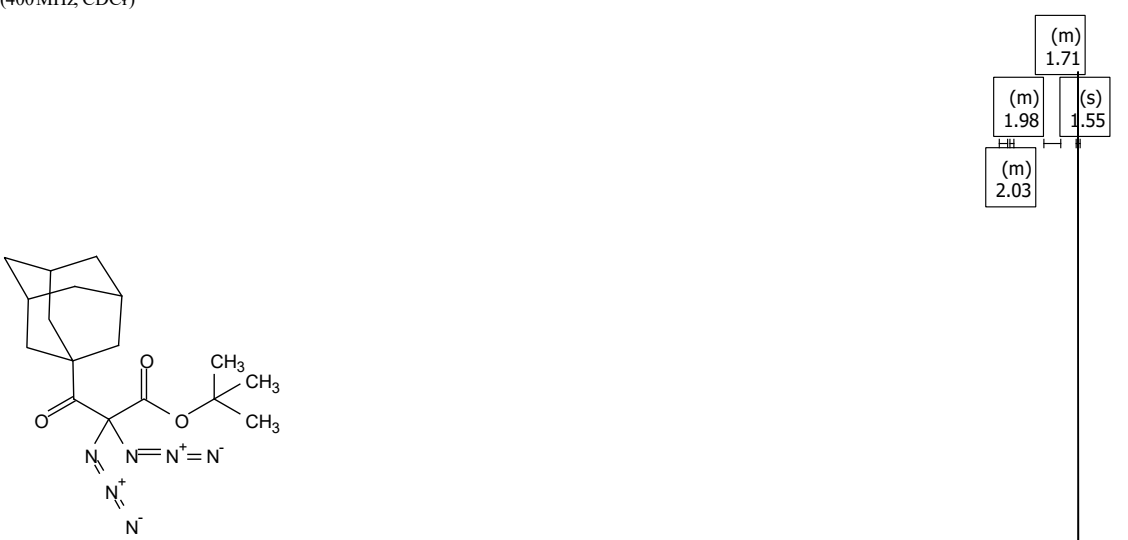

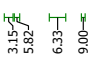

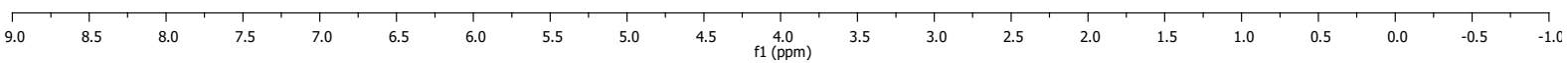



$\stackrel{n}{i}$

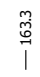

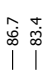
管

${ }^{13} \mathrm{C} \mathrm{NMR}(101 \mathrm{MHz}, \mathrm{CDCl})$
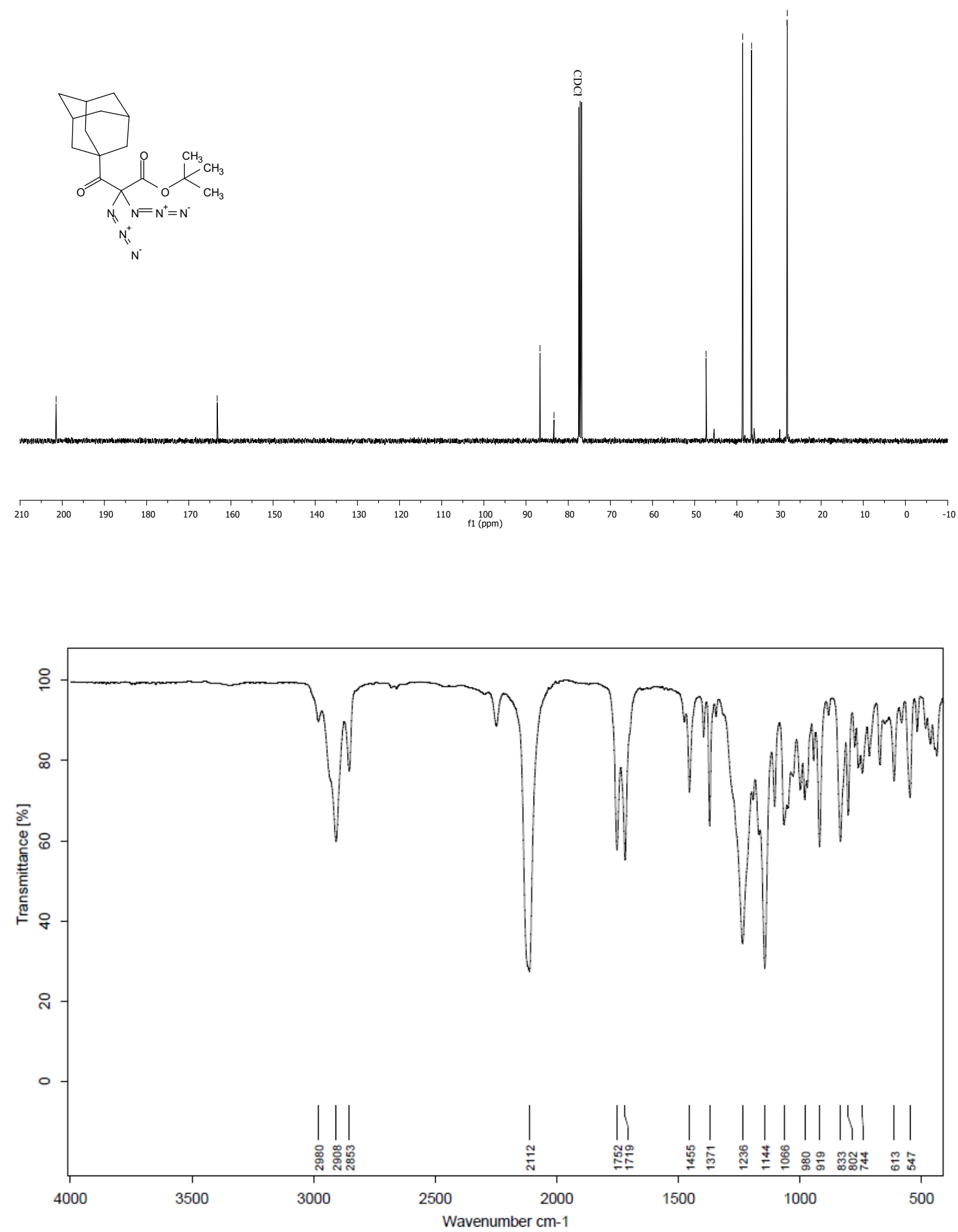
tert-Butyl 3-((3r,5r,7r)-adamantan-1-yl)-2,2-bis(4,5,6,7,8,9-hexahydro-1H-cycloocta[d] [1,2,3]triazol-1-yl)3-oxopropanoate

${ }^{1} \mathrm{H} \mathrm{NMR}(400 \mathrm{MHz}, \mathrm{CDCl})$
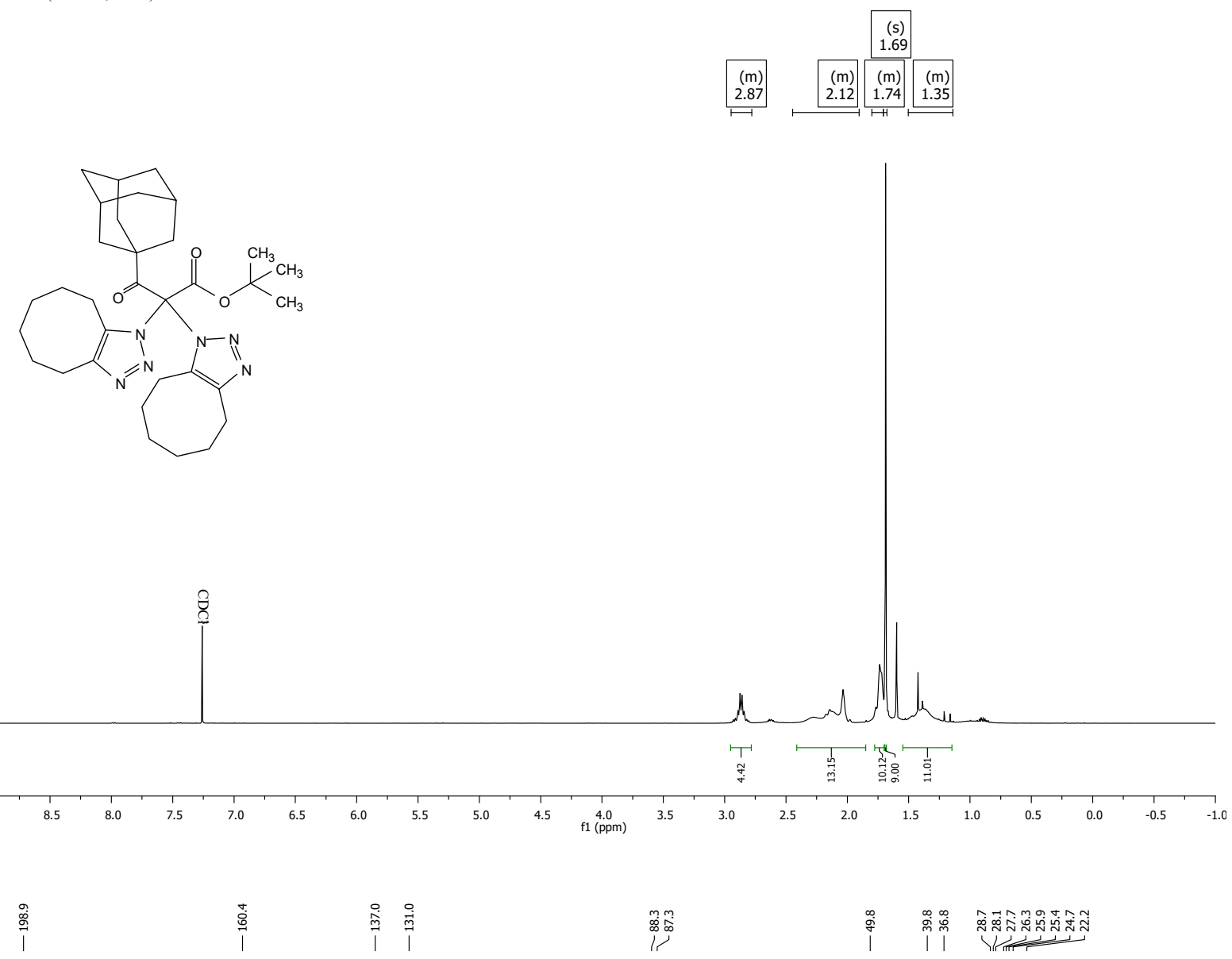

${ }^{13} \mathrm{C}$ NMR $(101 \mathrm{MHz}, \mathrm{CDCl})$
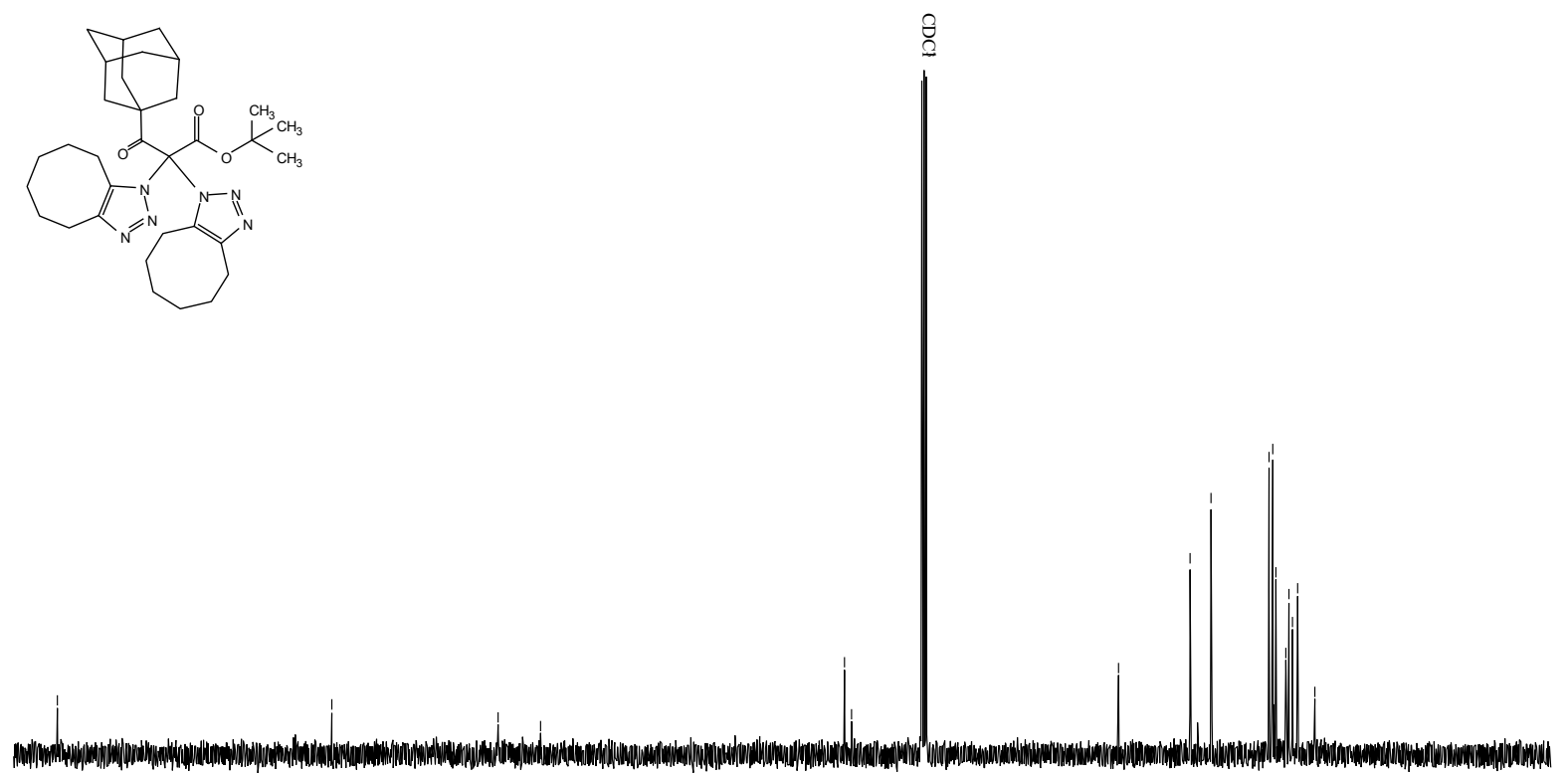

200

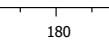

$170 \quad 160$

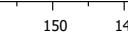

130

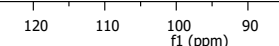

80 
tert-Butyl 2,2-diazido-3-oxo-3-phenylpropanoate (2m)

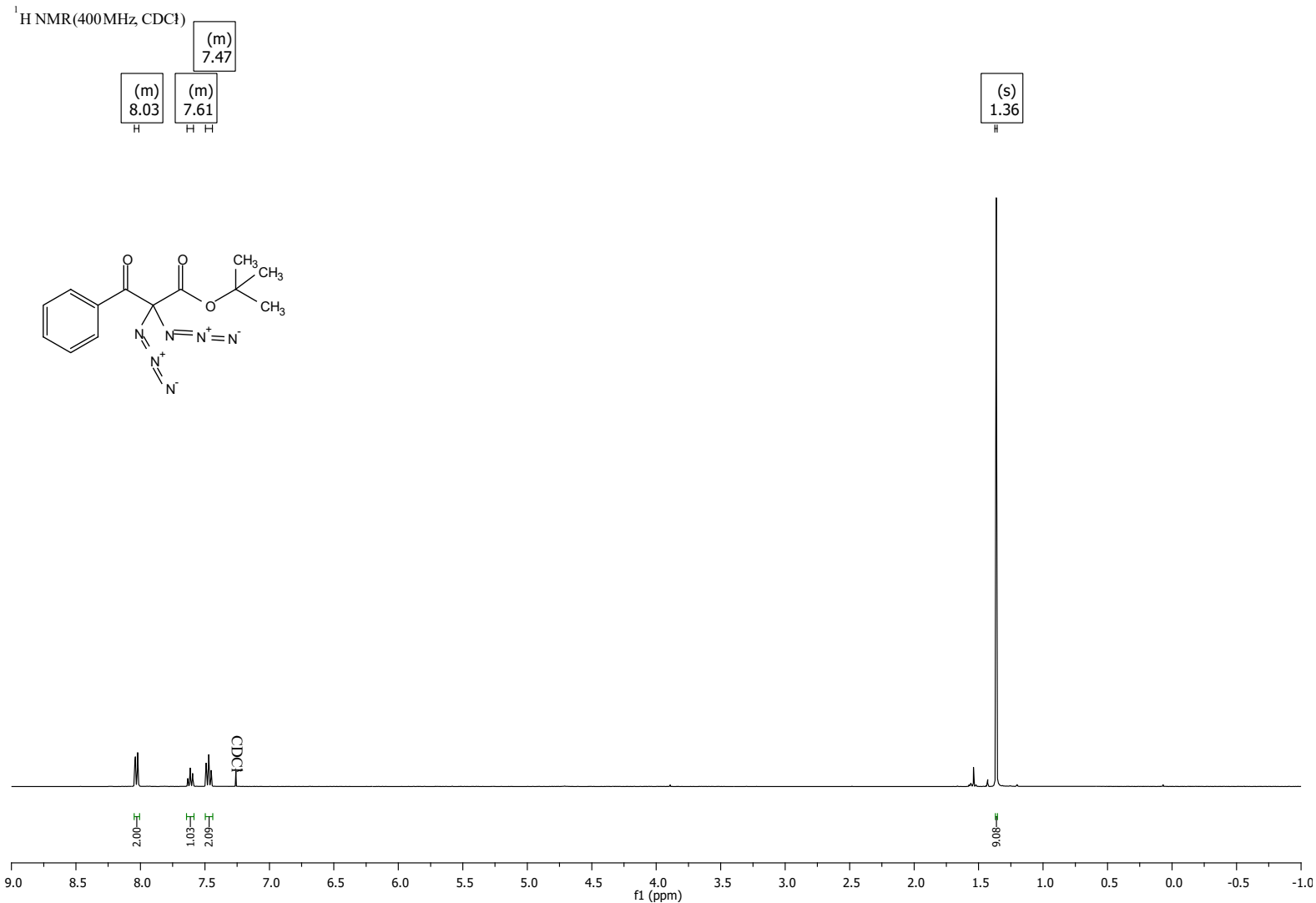

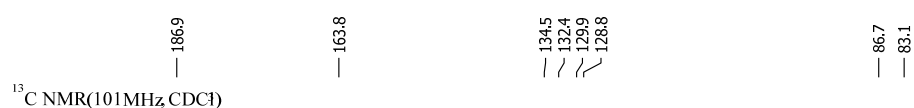
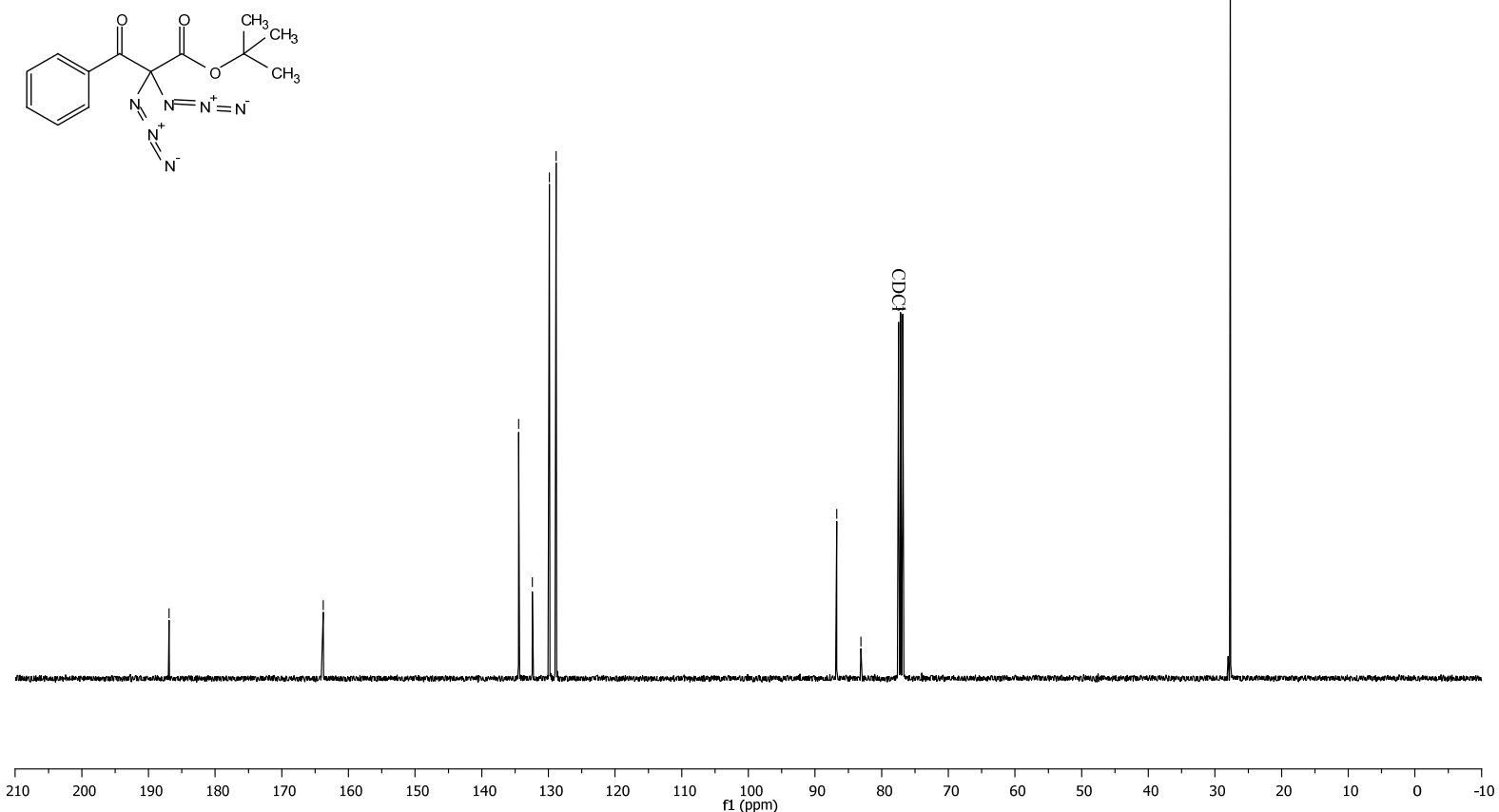


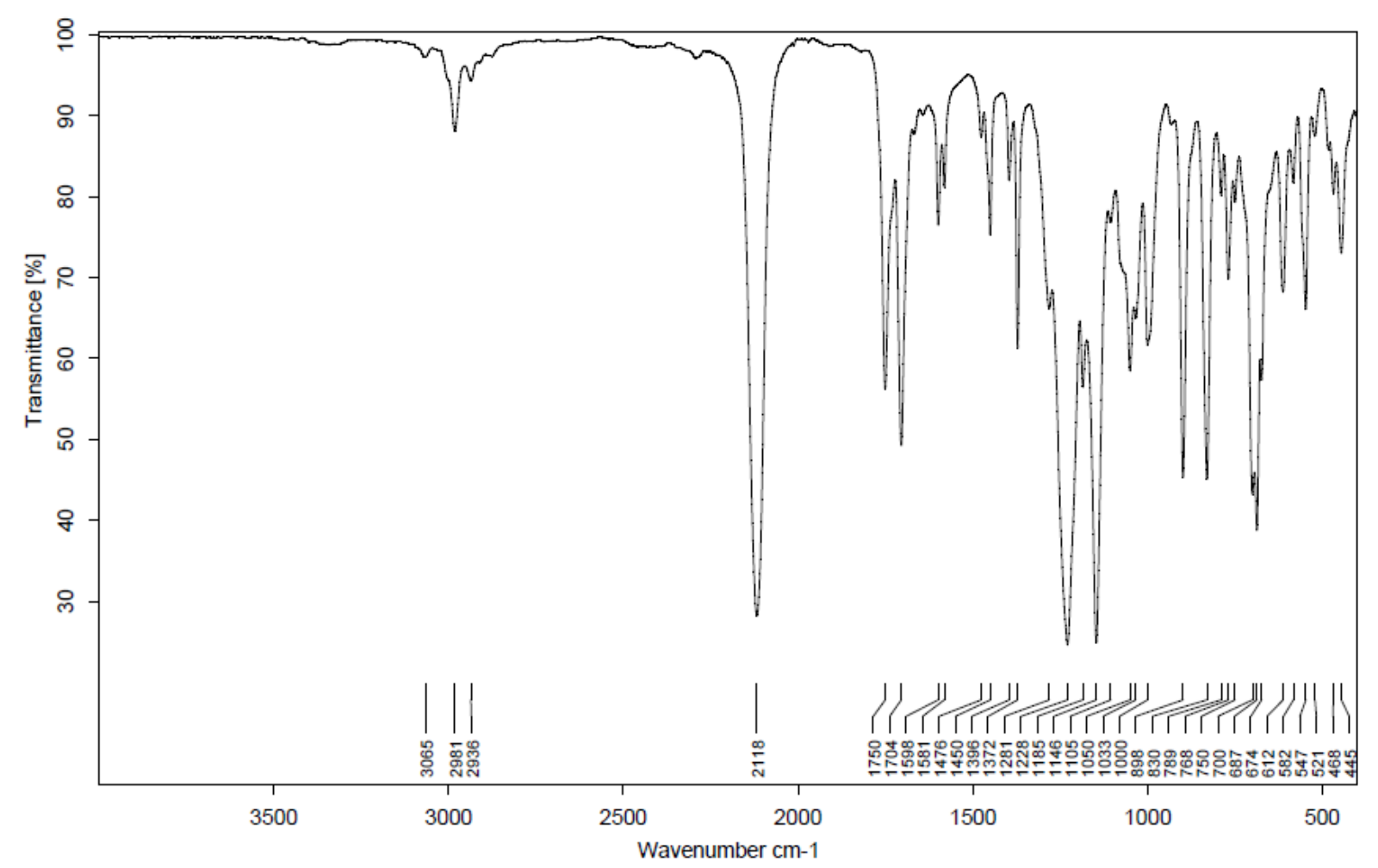

tert-Butyl 3-oxo-3-phenyl-2,2-bis(4-phenyl-1H-1,2,3-triazol-1-yl)propanoate

${ }^{1} \mathrm{H} \mathrm{NMR}(400 \mathrm{MHz}, \mathrm{CDCl})$

\begin{tabular}{|c|}
\hline$(s)$ \\
8.49 \\
\hline 4
\end{tabular}

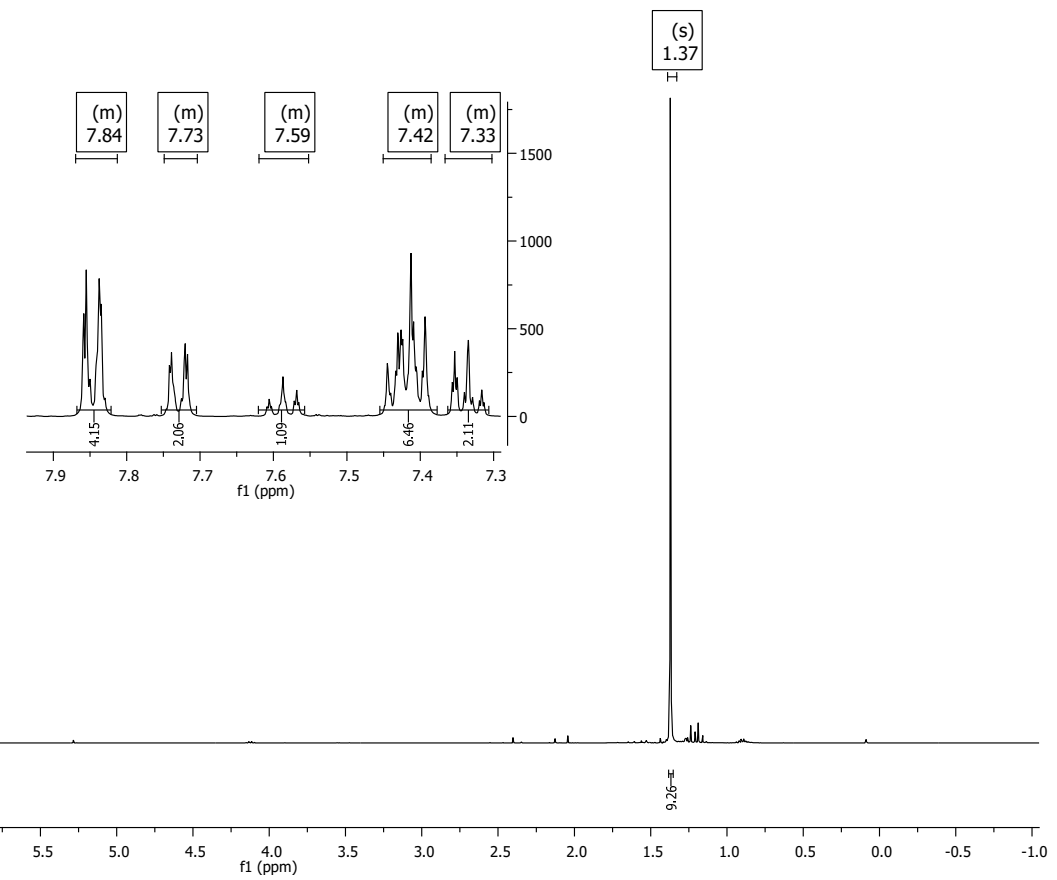




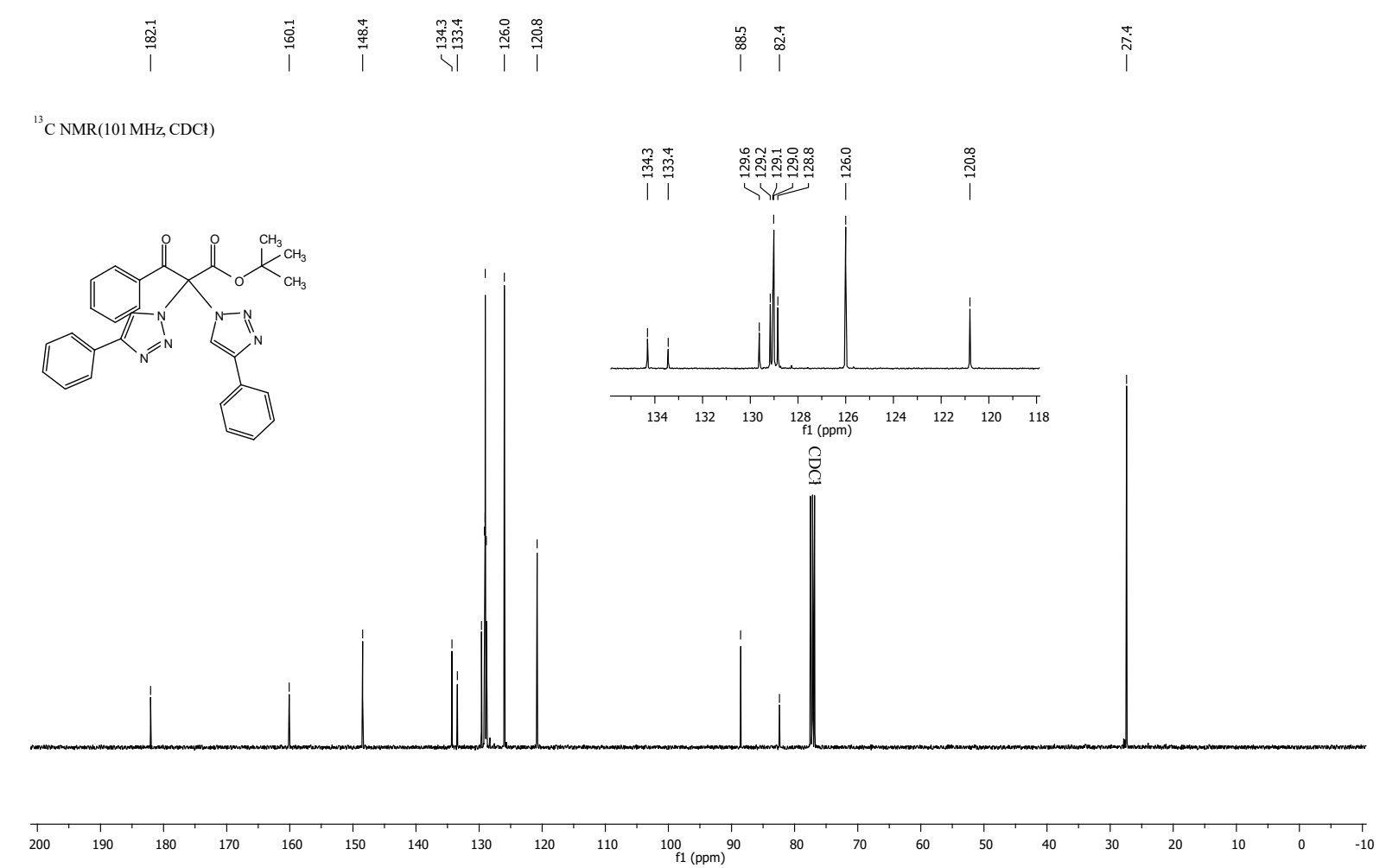

tert-Butyl 2,2-diazido-3-oxo-3-(p-tolyl)propanoate (2n)

${ }^{1} \mathrm{H} \mathrm{NMR}(600 \mathrm{MHz}, \mathrm{CDCl})$
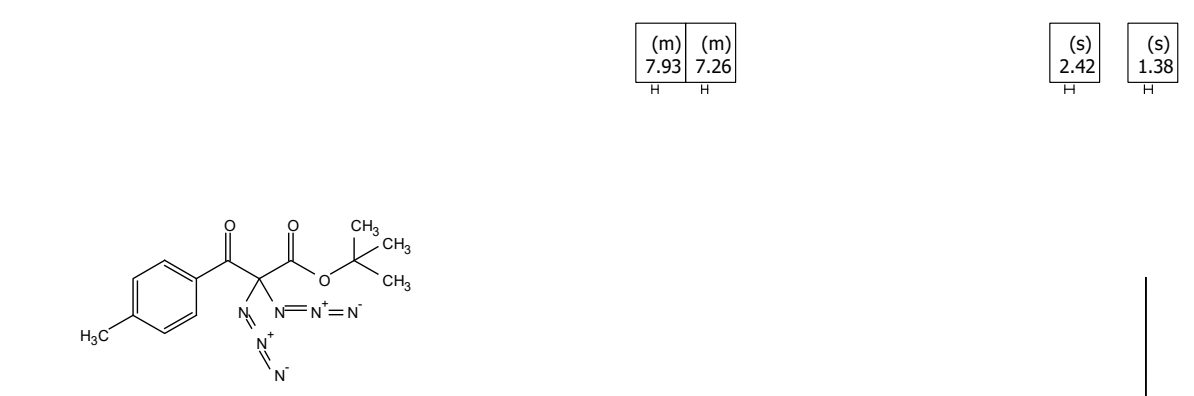

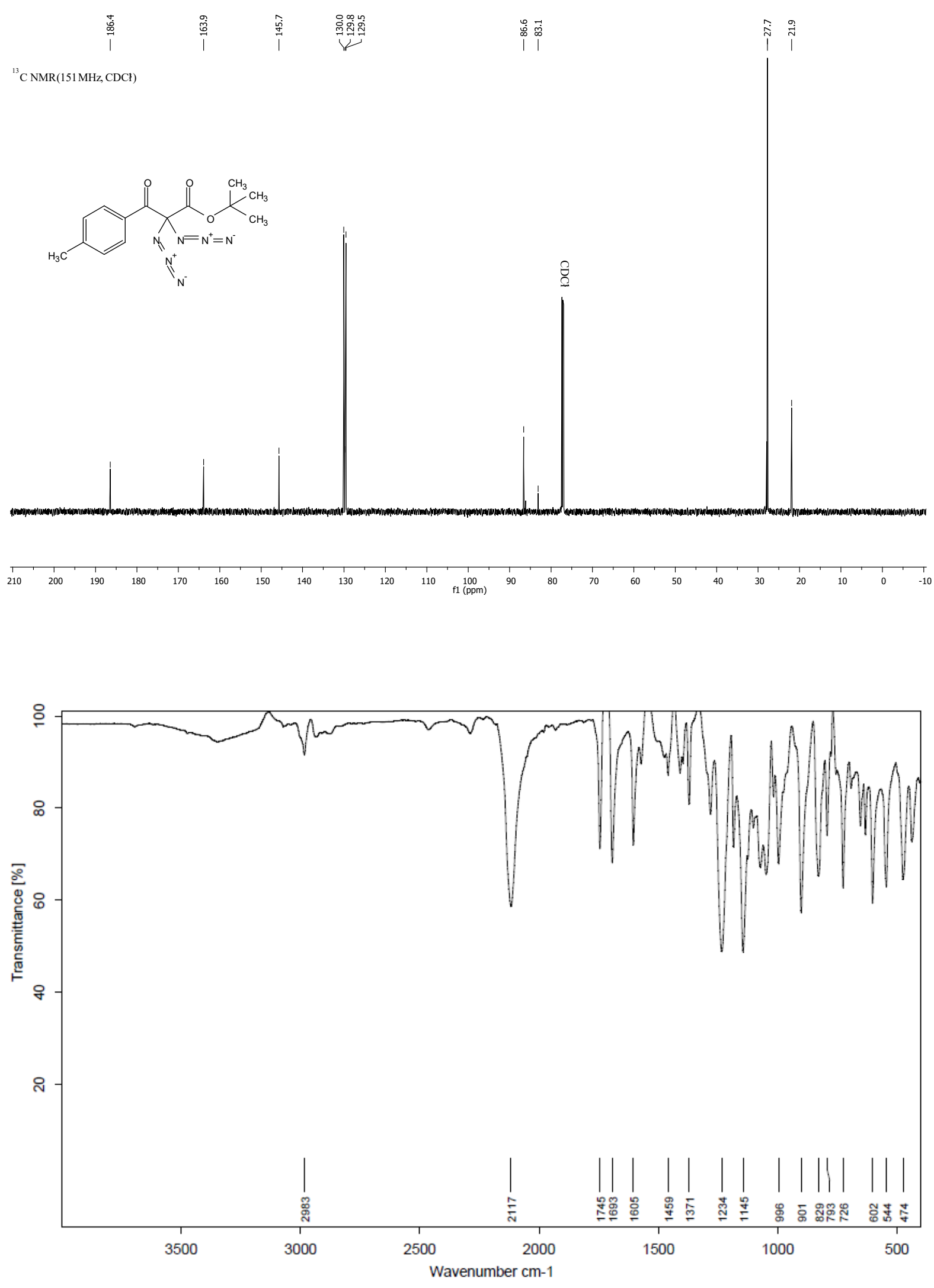
tert-Butyl 2,2-bis(4,5,6,7,8,9-hexahydro-1H-cycloocta[d][1,2,3]triazol-1-yl)-3-oxo-3-(ptolyl)propanoate
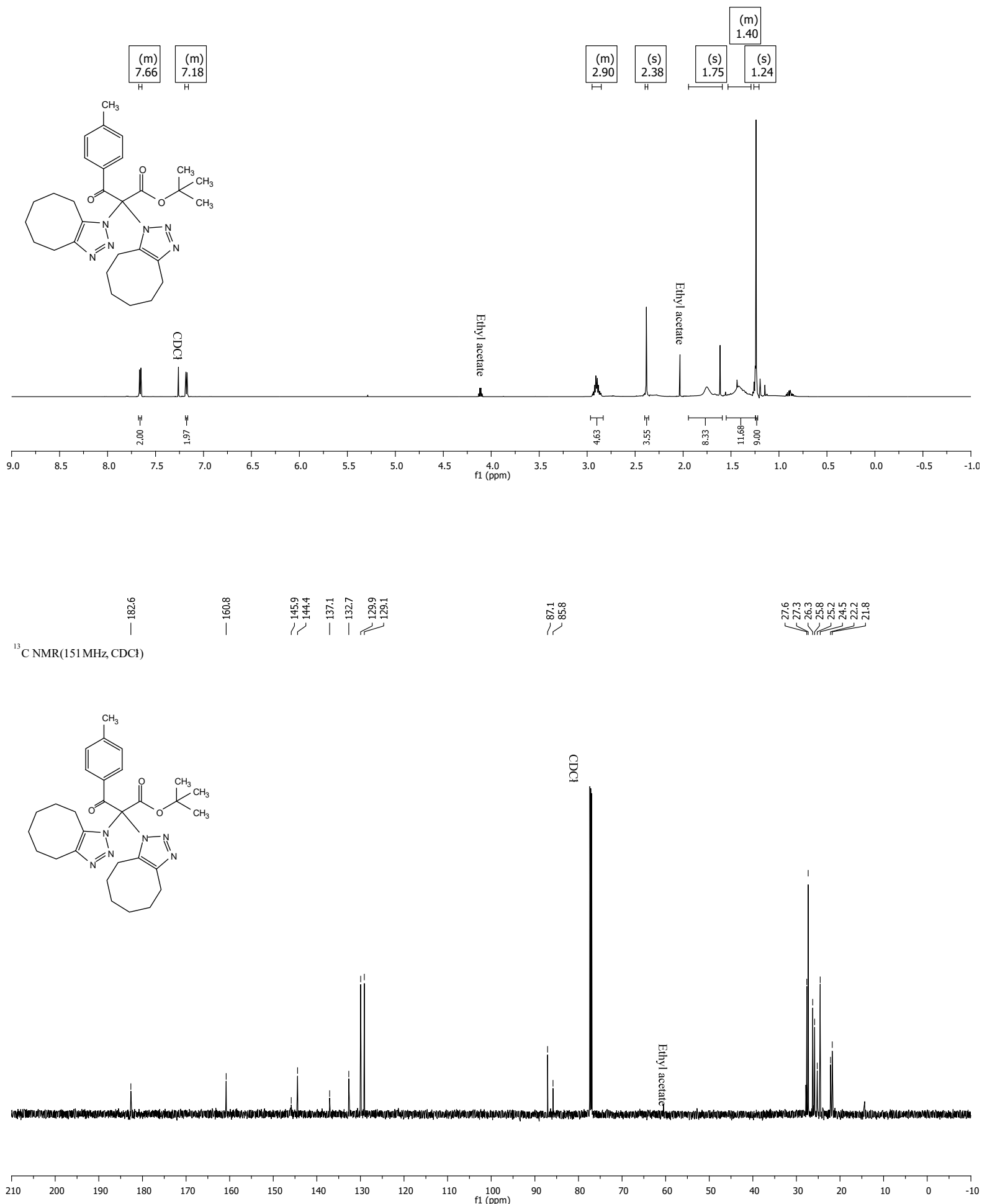
tert-Butyl 2,2-diazido-3-(4-chlorophenyl)-3-oxopropanoate (2o)

${ }^{1} \mathrm{H}$ NMR(600MHz, CDCr)

\begin{tabular}{|l|l|}
\hline$(\mathrm{m})$ & $(\mathrm{m})$ \\
7.98 & 7.45 \\
\hline &
\end{tabular}

\begin{tabular}{r|}
$(\mathrm{s})$ \\
1.39 \\
\hline
\end{tabular}
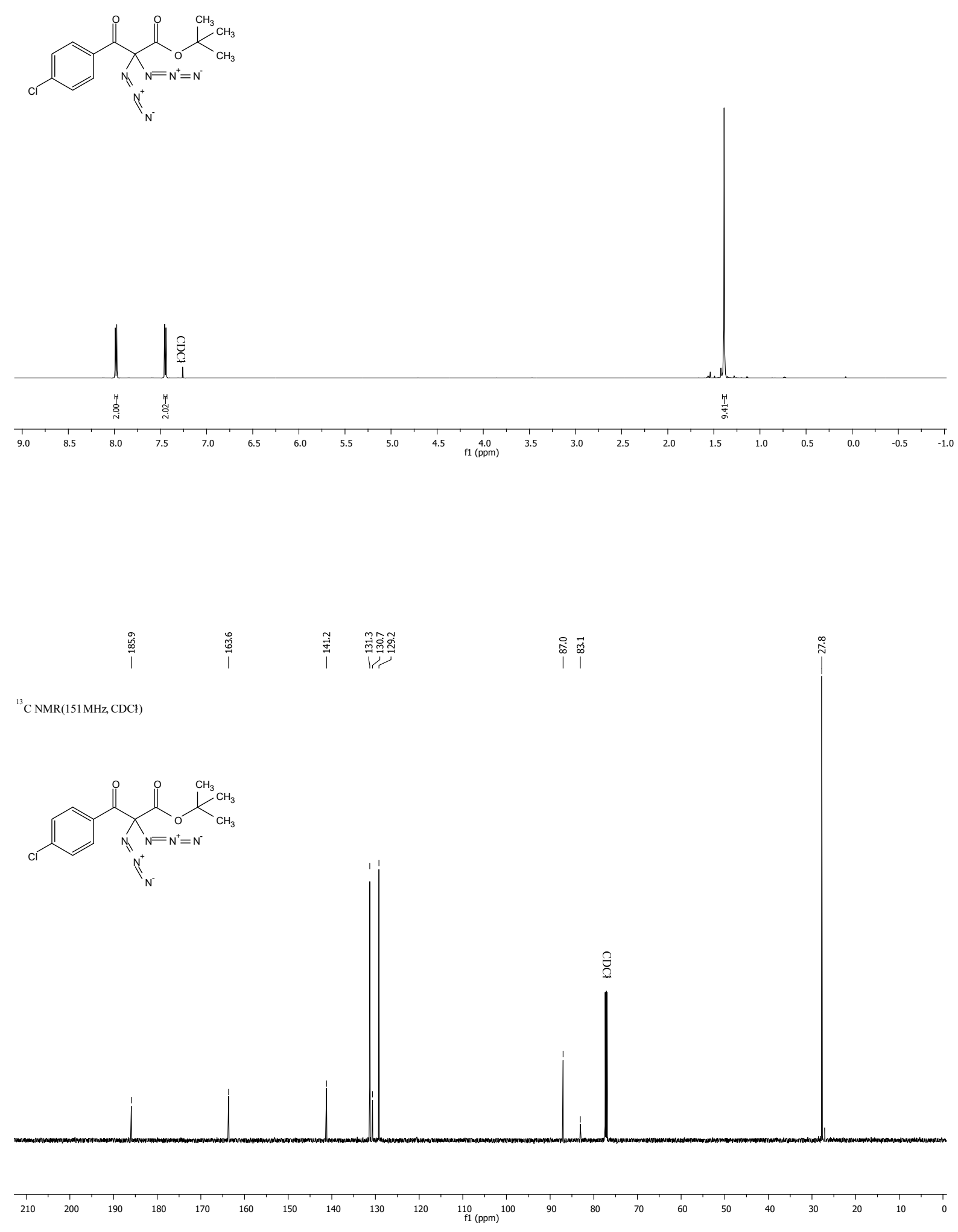

33 


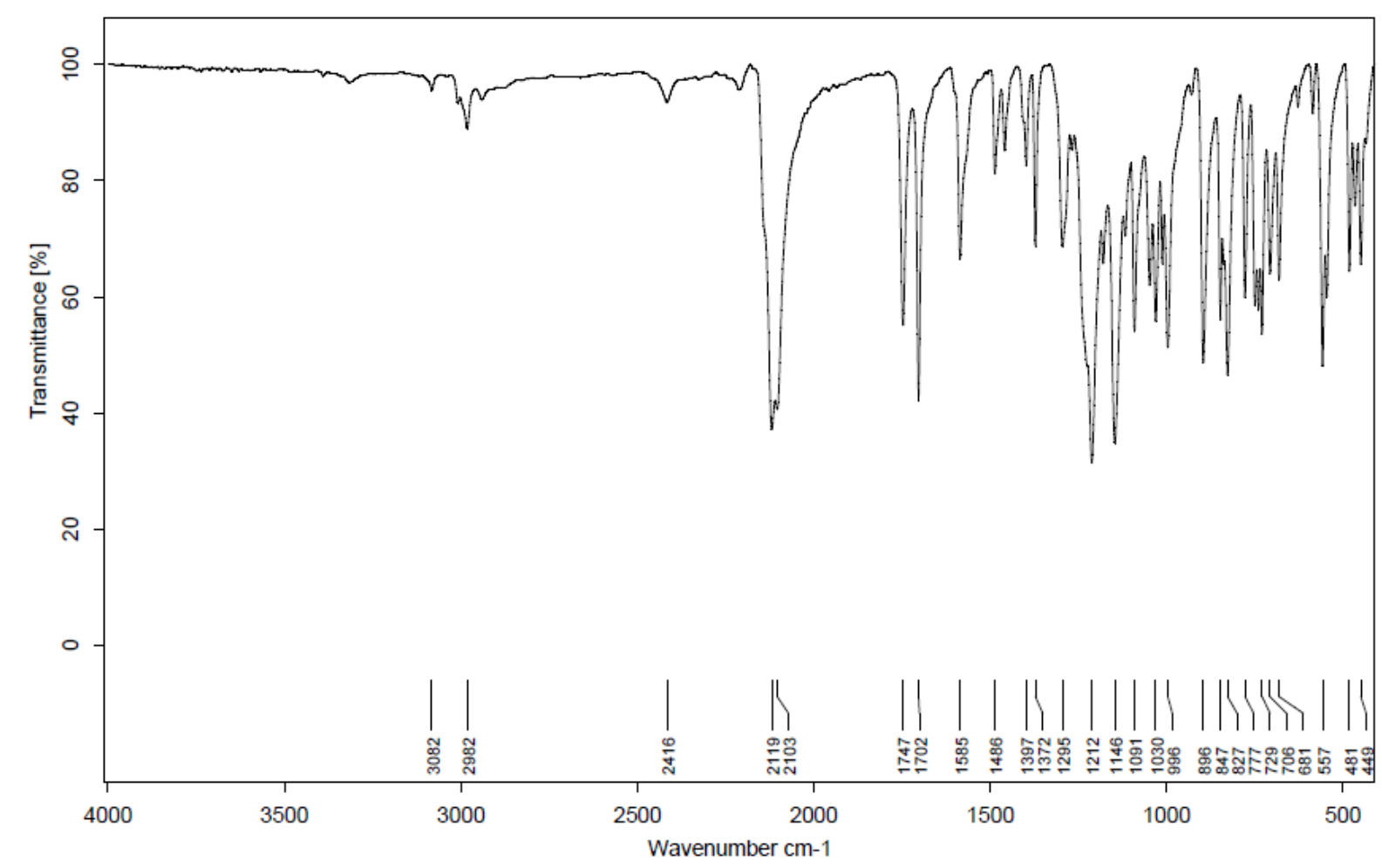

tert-Butyl 3-(4-chlorophenyl)-2,2-bis(4,5,6,7,8,9-hexahydro-1H-cycloocta[d][1,2,3]triazol-1-yl)-3oxopropanoate
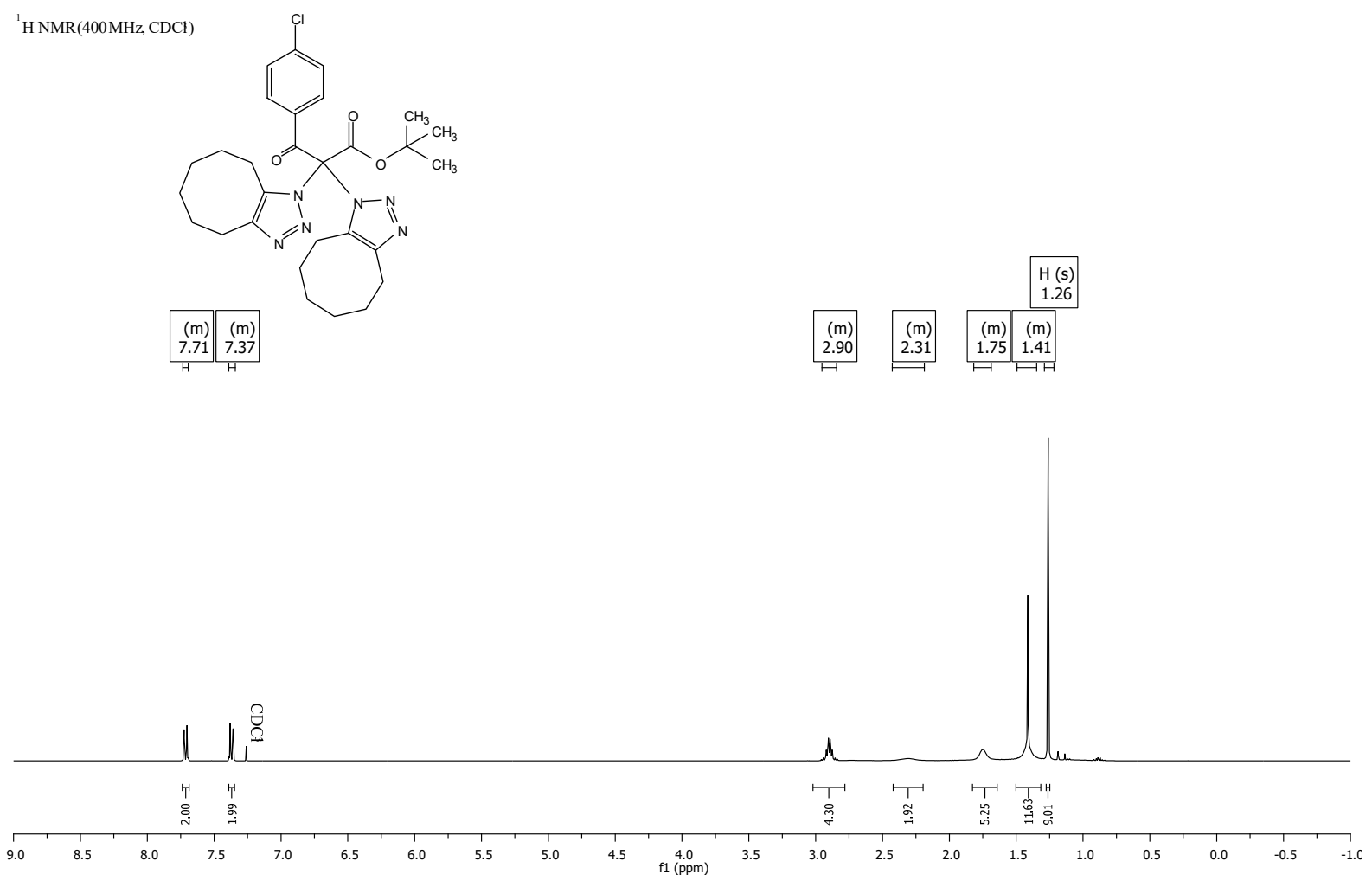

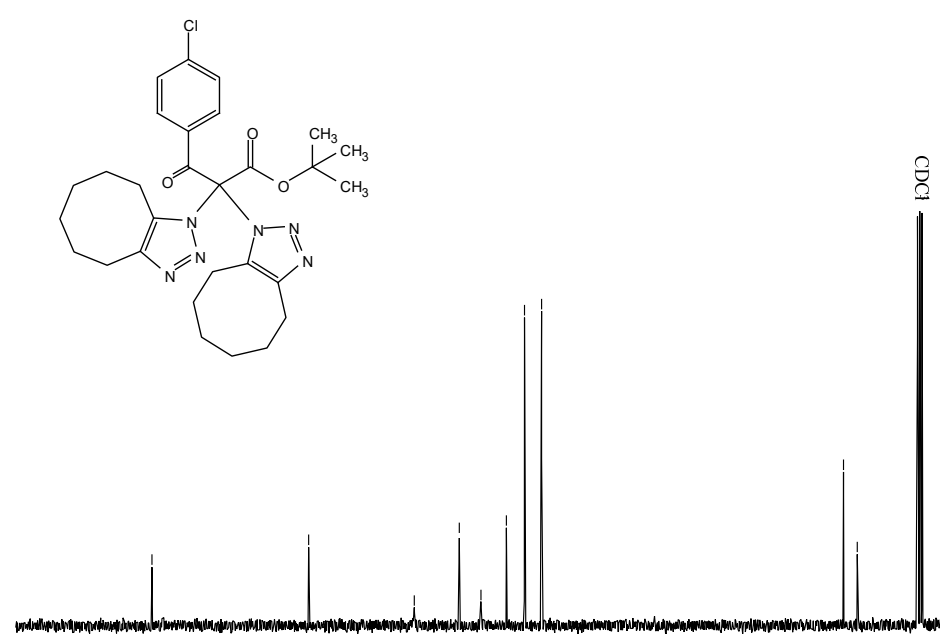

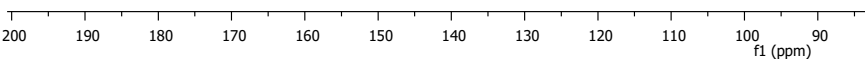

tert-Butyl 2,2-diazido-3-(4-methoxyphenyl)-3-oxopropanoate (2p)

H NMR(600MHz, CDCl)<smiles>COc1ccc(C(=O)C(N=[N+]=[N-])(N=[N+]=[N-])C(=O)OC(C)(C)C)cc1</smiles><smiles>C1C[C@H]2CC[C@@H]12</smiles>

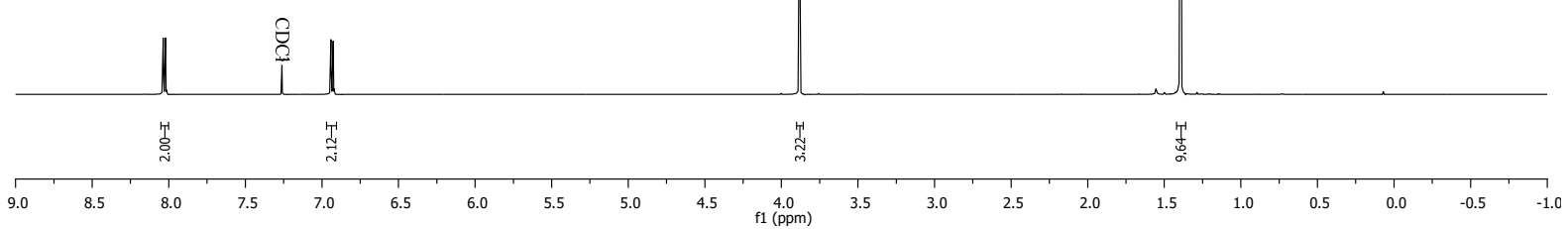




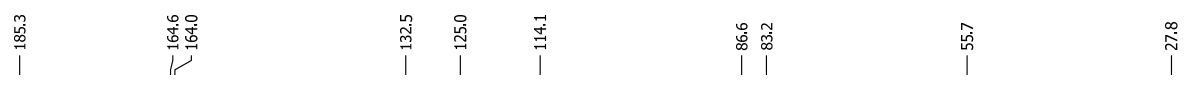

${ }^{13} \mathrm{C} \mathrm{NMR}(151 \mathrm{MHz}, \mathrm{CDC})$.
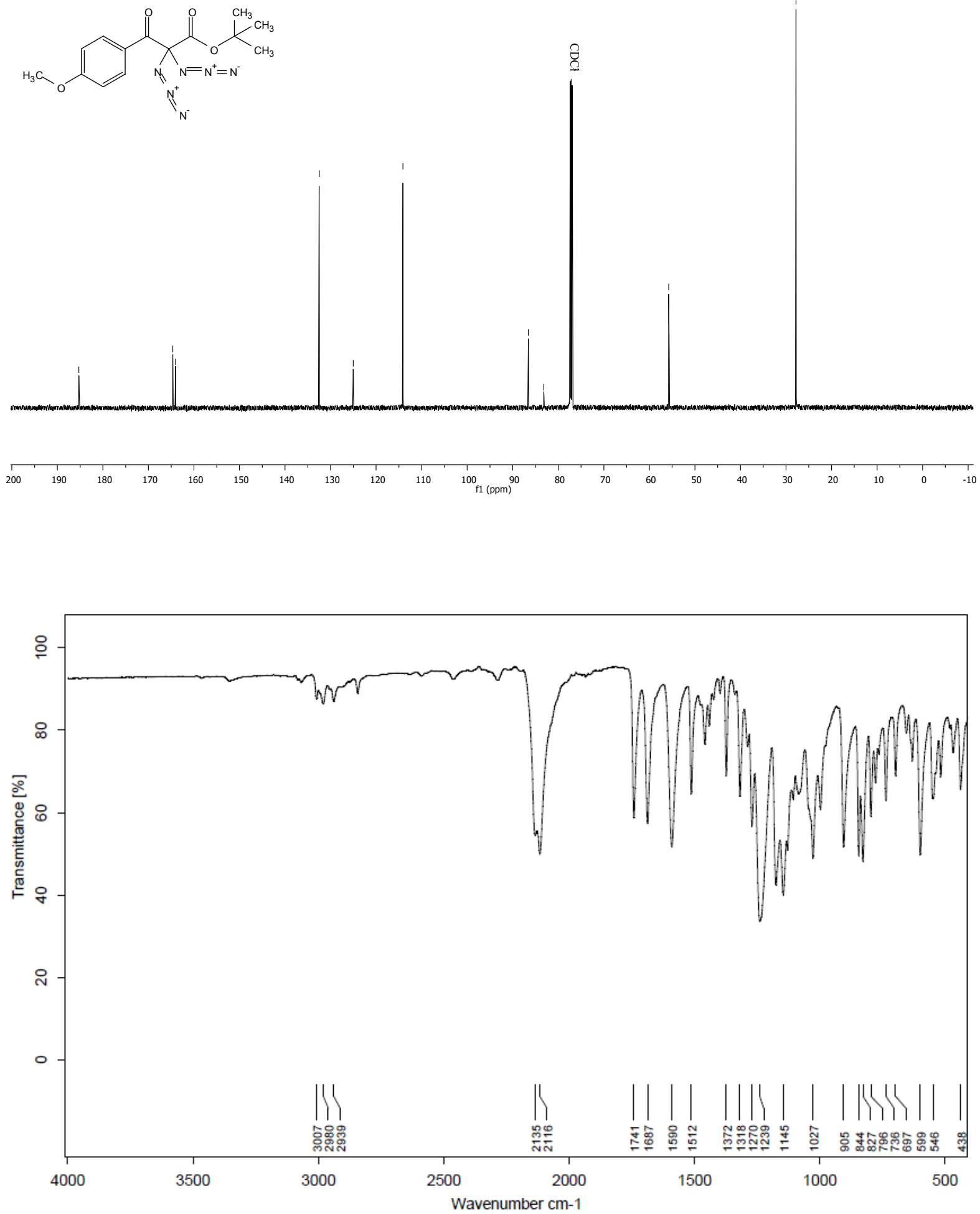
tert-Butyl 2,2-bis(4,5,6,7,8,9-hexahydro-1H-cycloocta[d][1,2,3]triazol-1-yl)-3-(4-methoxyphenyl)3-oxopropanoate

${ }^{1} \mathrm{H} \mathrm{NMR}(400 \mathrm{MHz}, \mathrm{CDCl})$

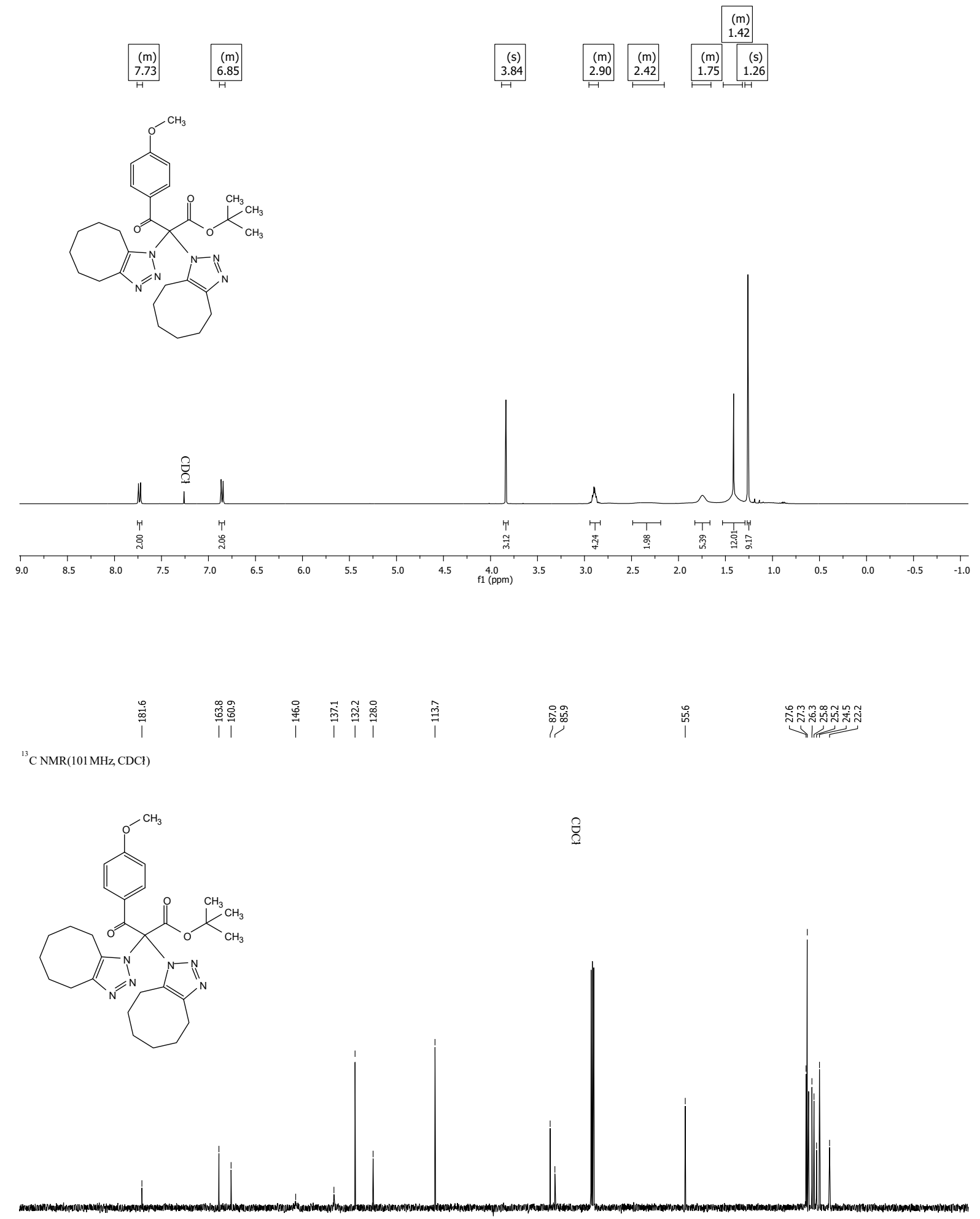

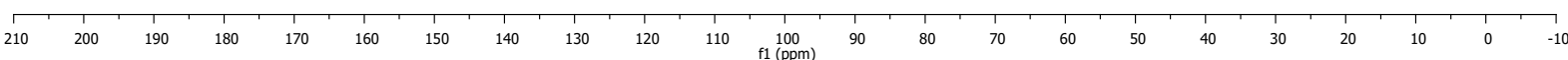


tert-Butyl 2,2-diazido-3-(furan-2-yl)-3-oxopropanoate (2q)

H NMR $(600 \mathrm{MHz}, \mathrm{CDCl})$
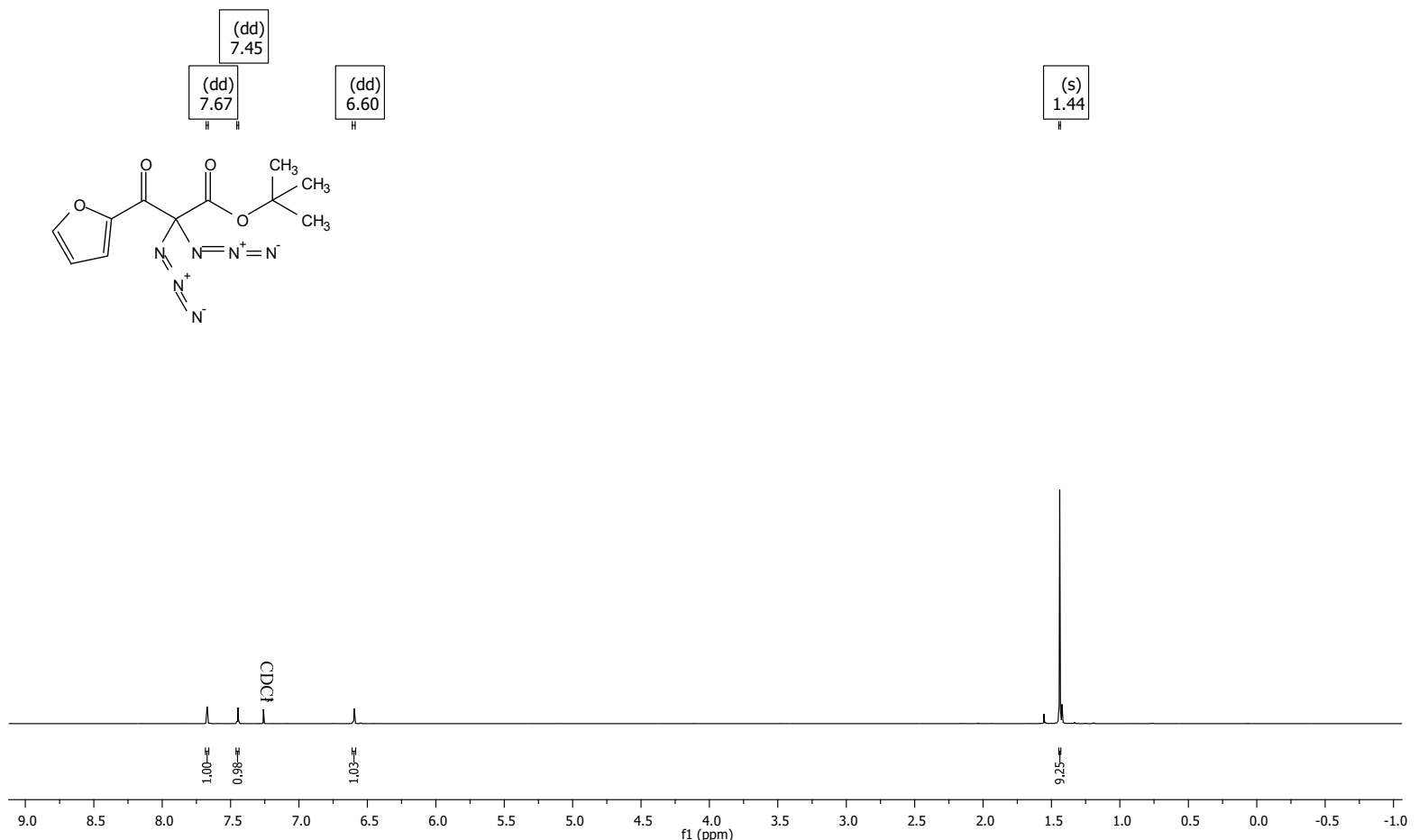

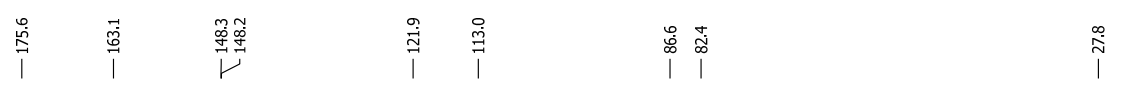

${ }^{13} \mathrm{C} \mathrm{NMR}(151 \mathrm{MHz}, \mathrm{CDC})$
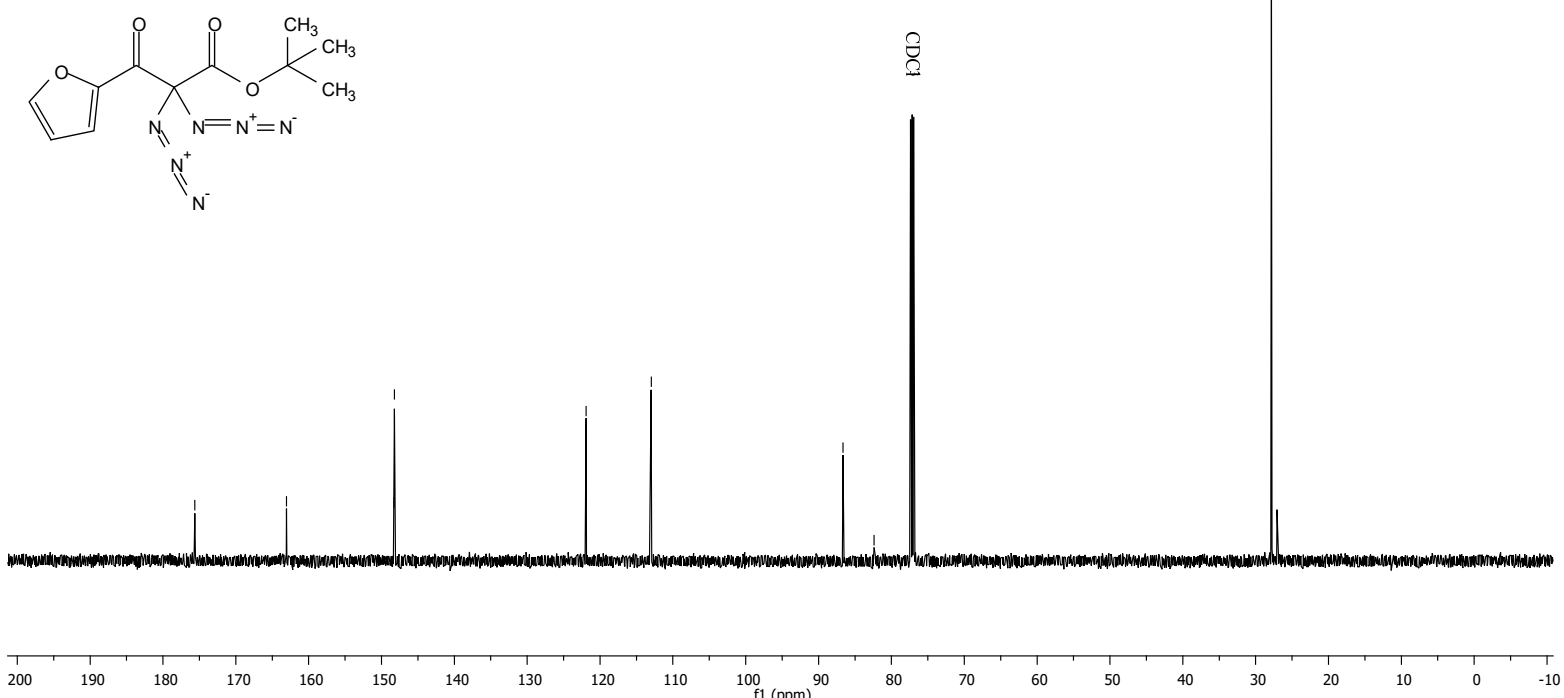


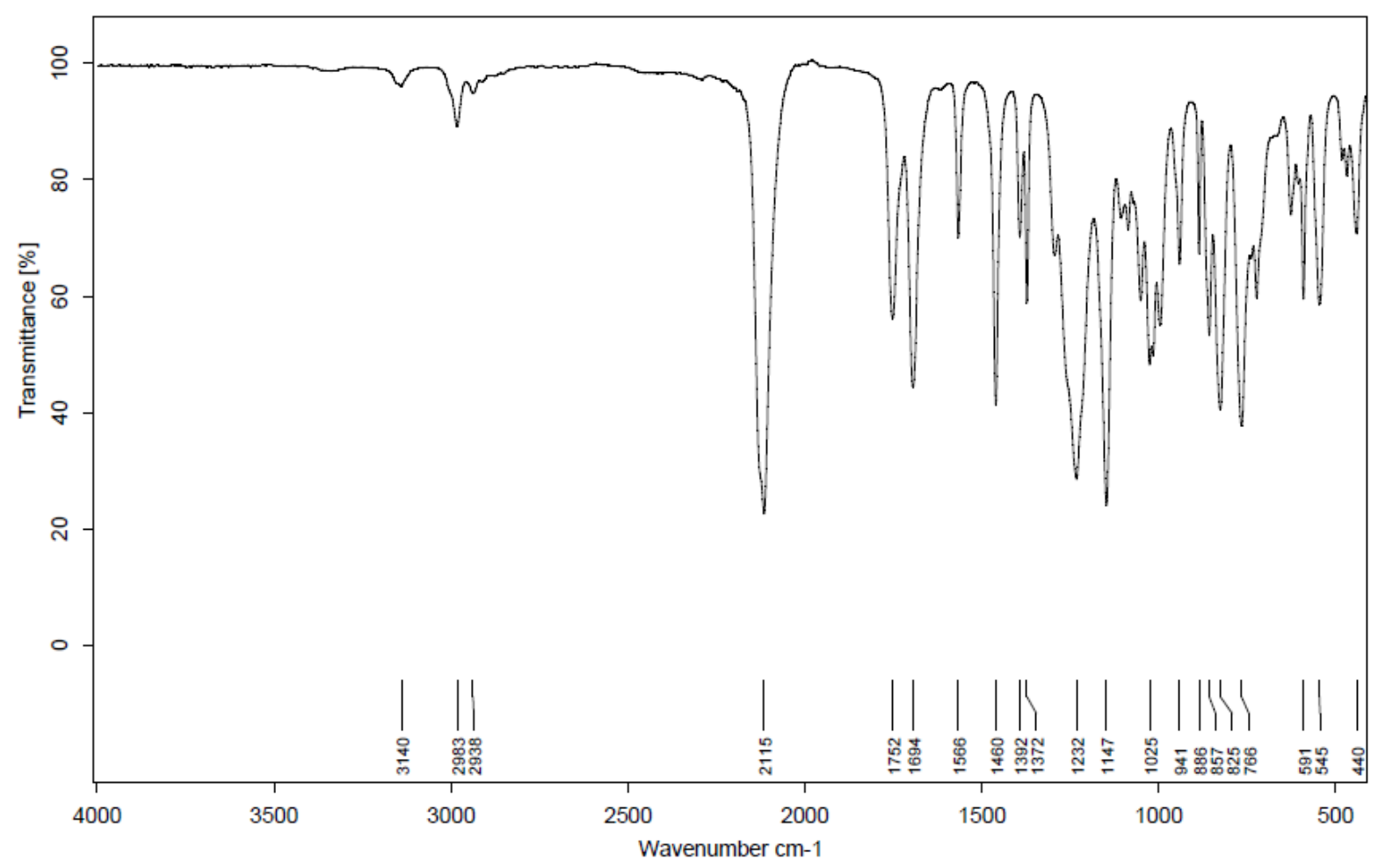

tert-Butyl 3-(furan-2-yl)-2,2-bis(4,5,6,7,8,9-hexahydro-1H-cycloocta[d] [1,2,3]triazol-1-yl)-3oxopropanoate

${ }^{1} \mathrm{H}$ NMR(400MHz, CDCl)
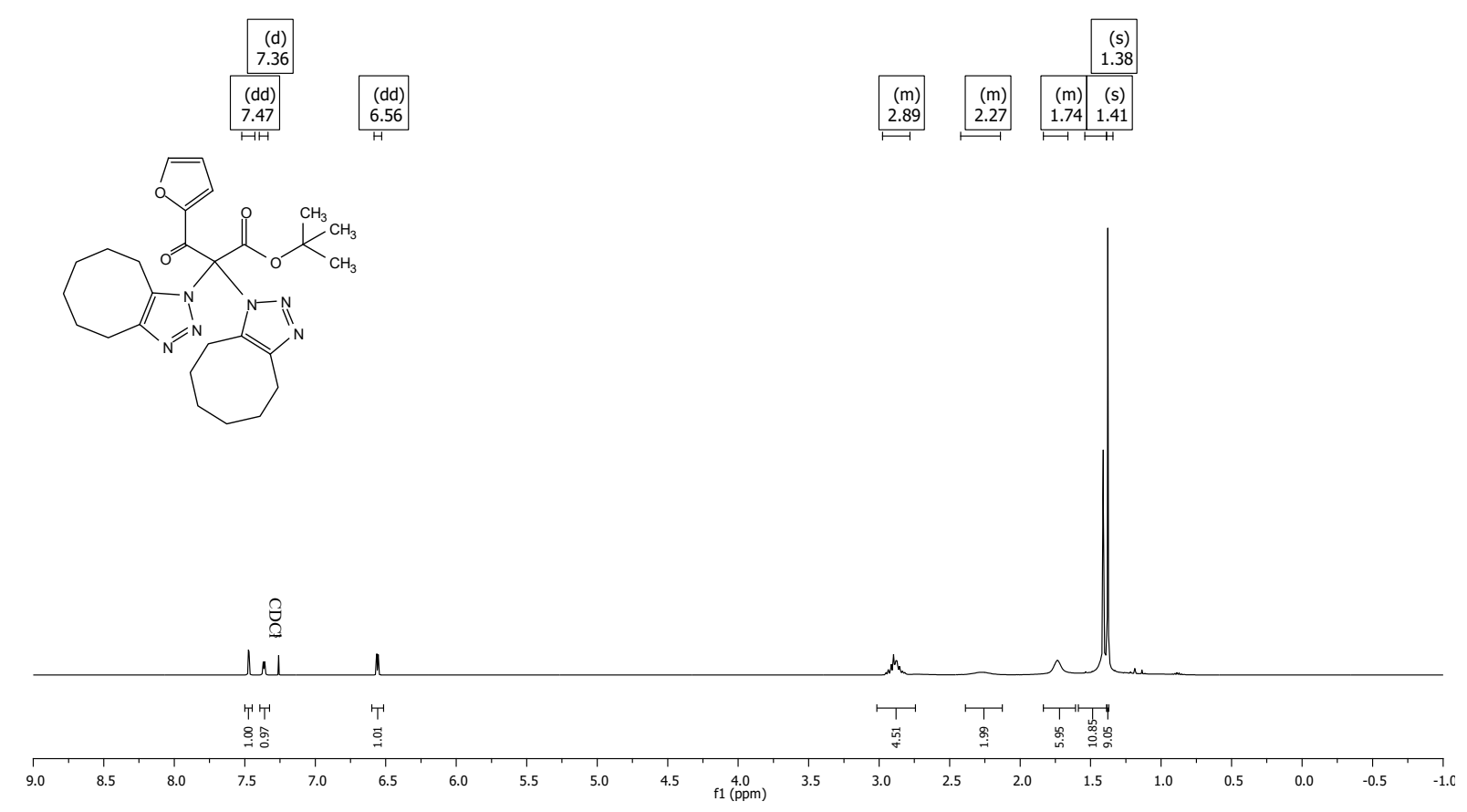


$$
\text { । }
$$

${ }^{13} \mathrm{C}$ NMR(101 MHz, CDCF)
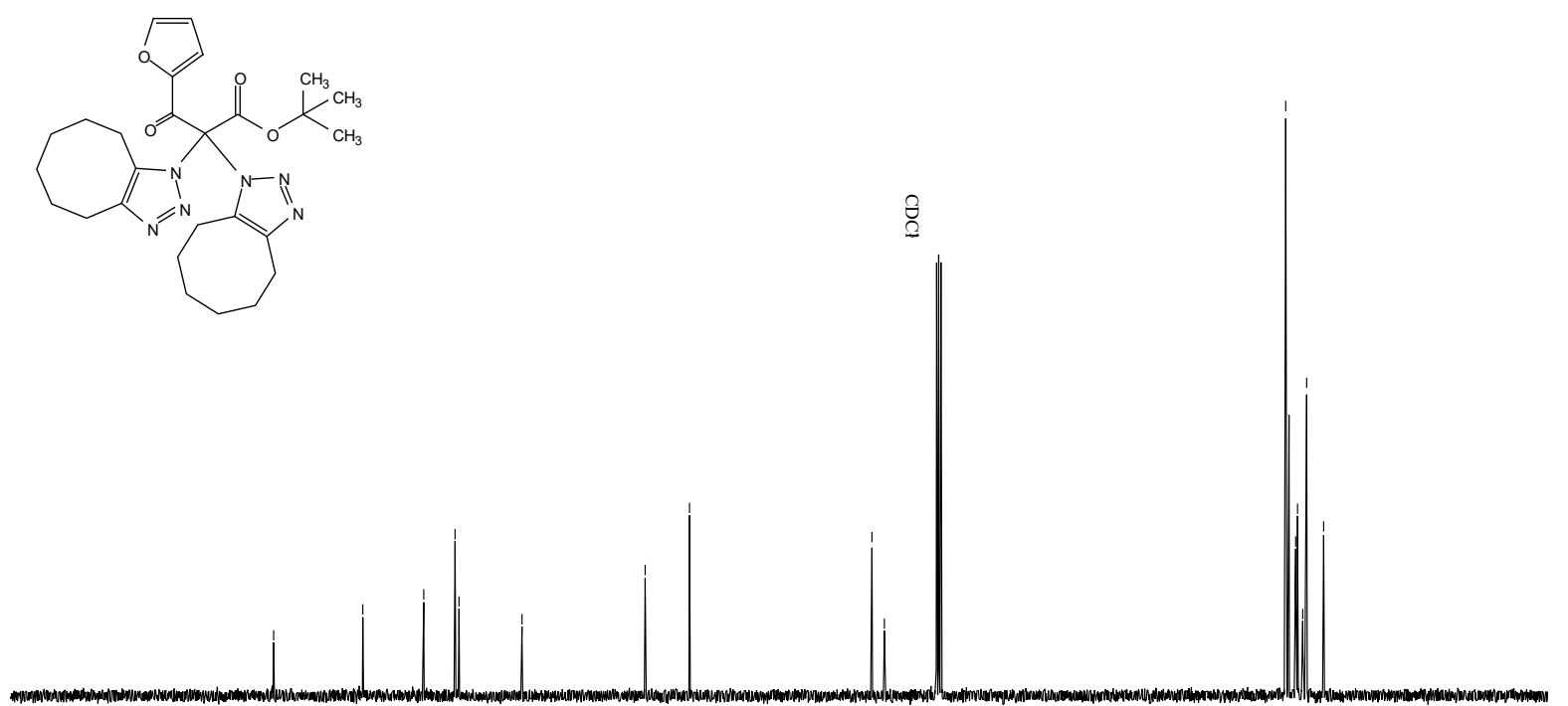

210

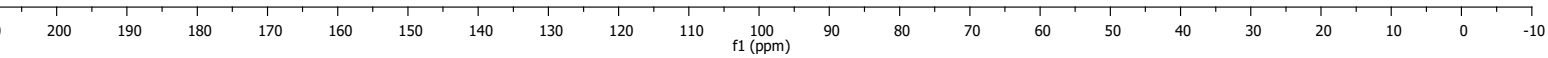

(E)-tert-Butyl 2,2-diazido-3-oxo-5-phenylpent-4-enoate (2r)

H NMR (400MHz, CDCl)
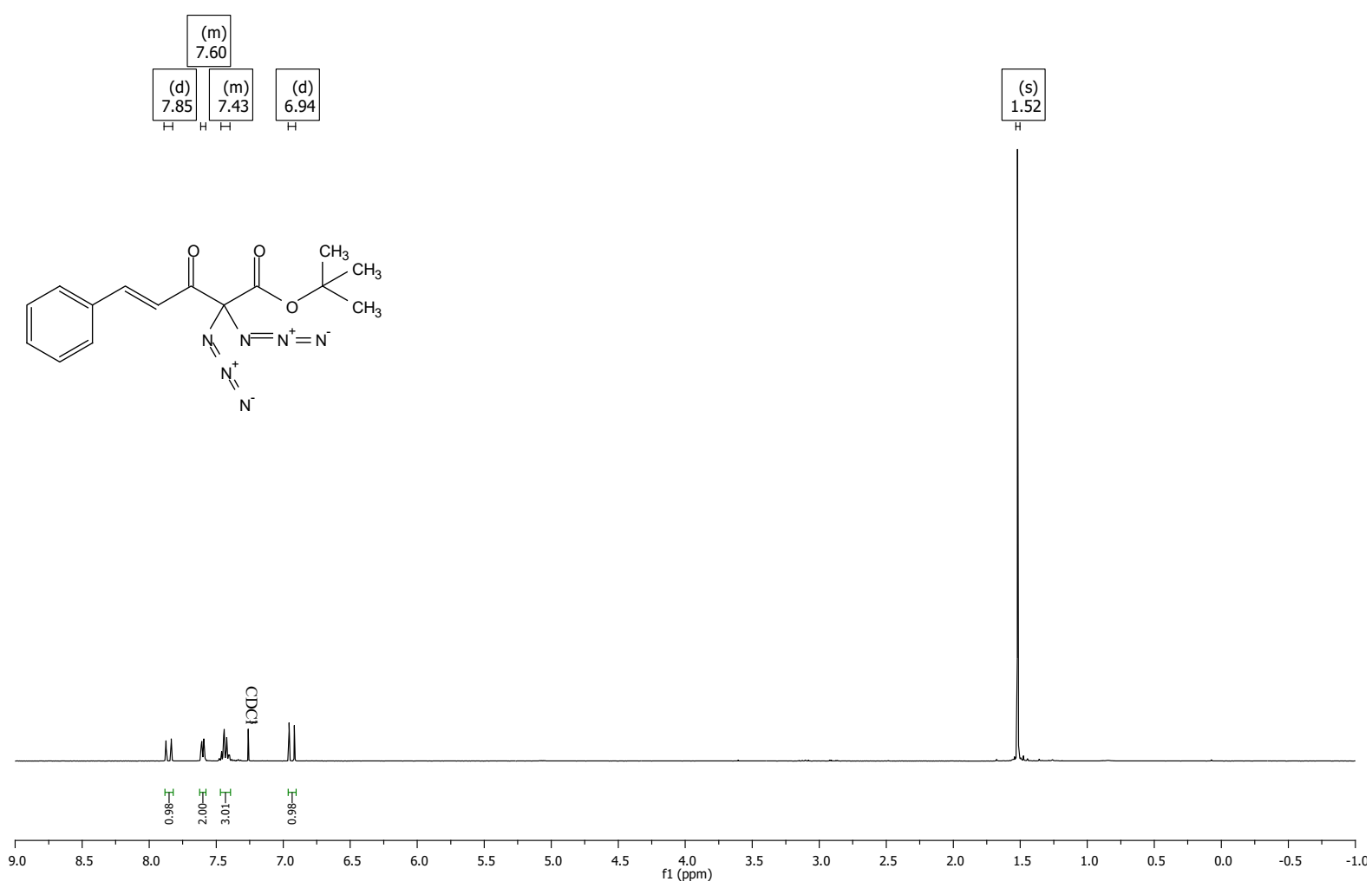


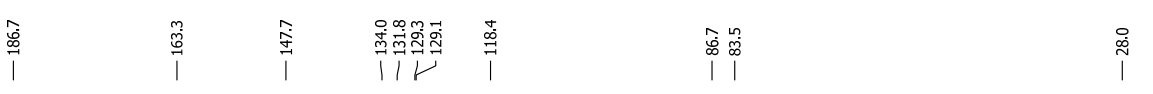

${ }^{3} \mathrm{C}$ NMR(101 MHz, CDCl)
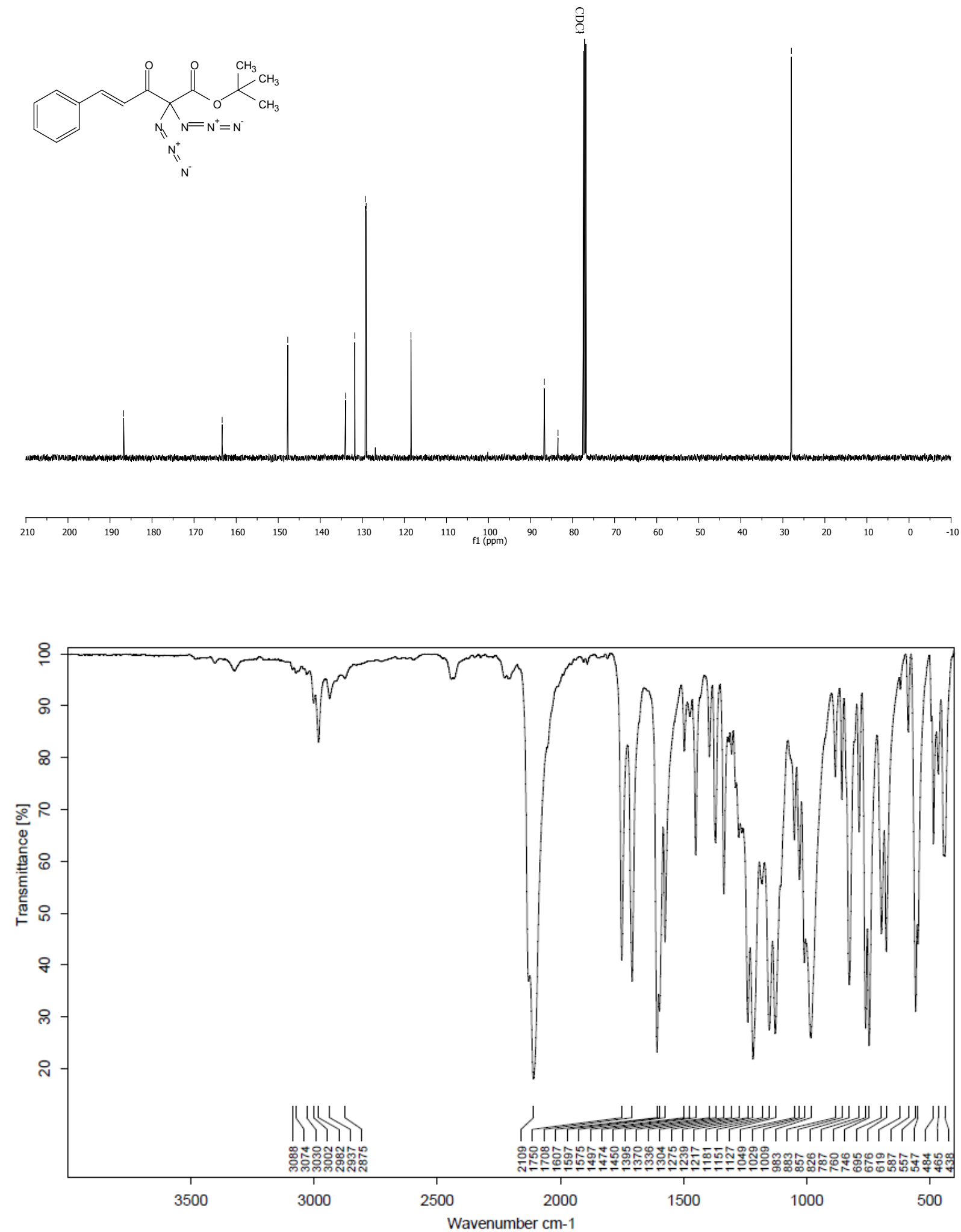
tert-Butyl 2,2-diazido-3-oxohept-6-enoate (2s)

${ }^{1} \mathrm{H}$ NMR (600 MHz, CDCl)

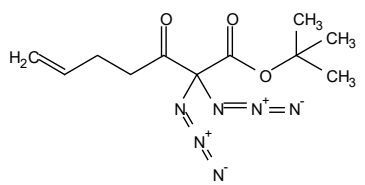

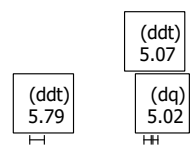
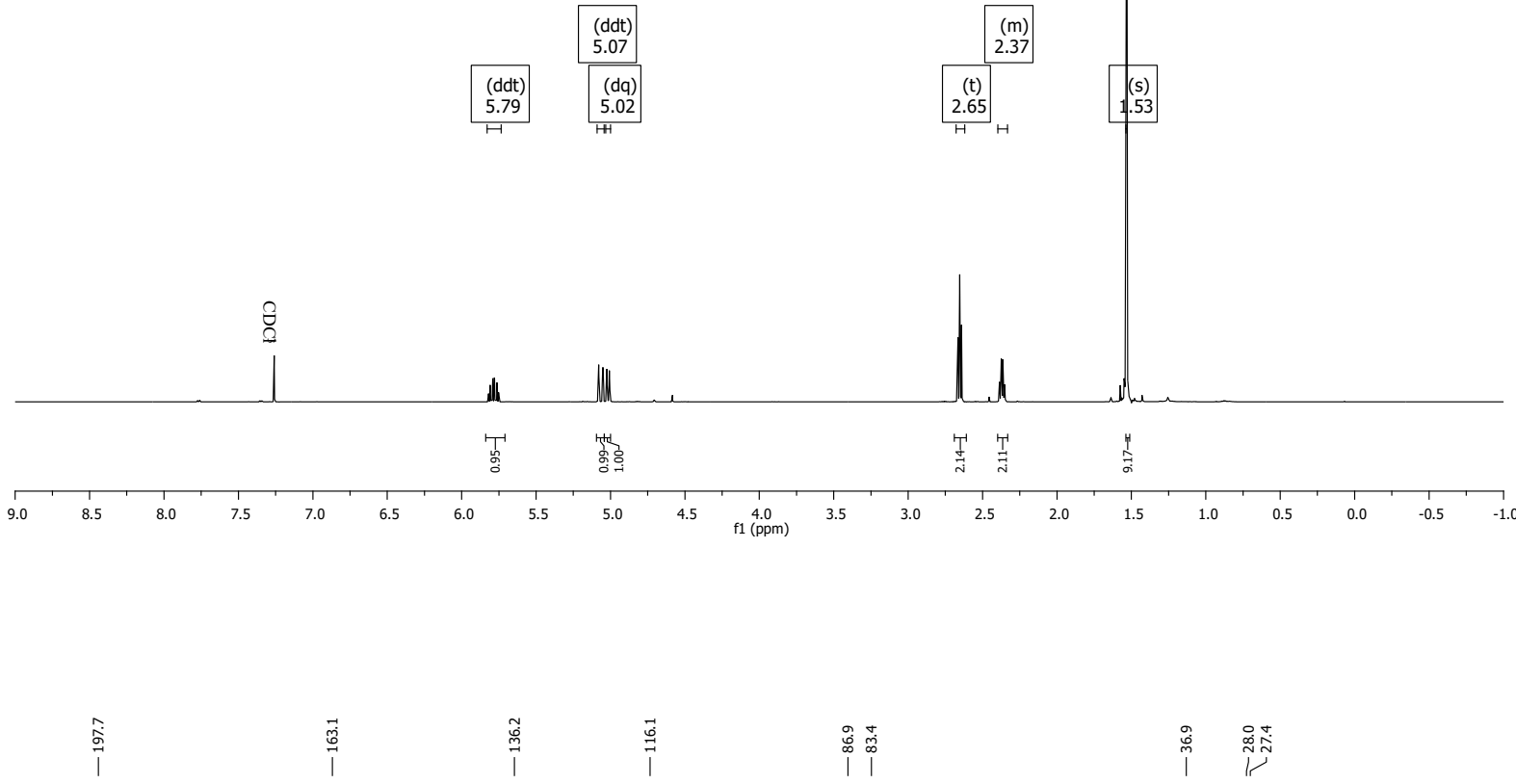

${ }^{13} \mathrm{C}$ NMR(151 MHz, CDCF)
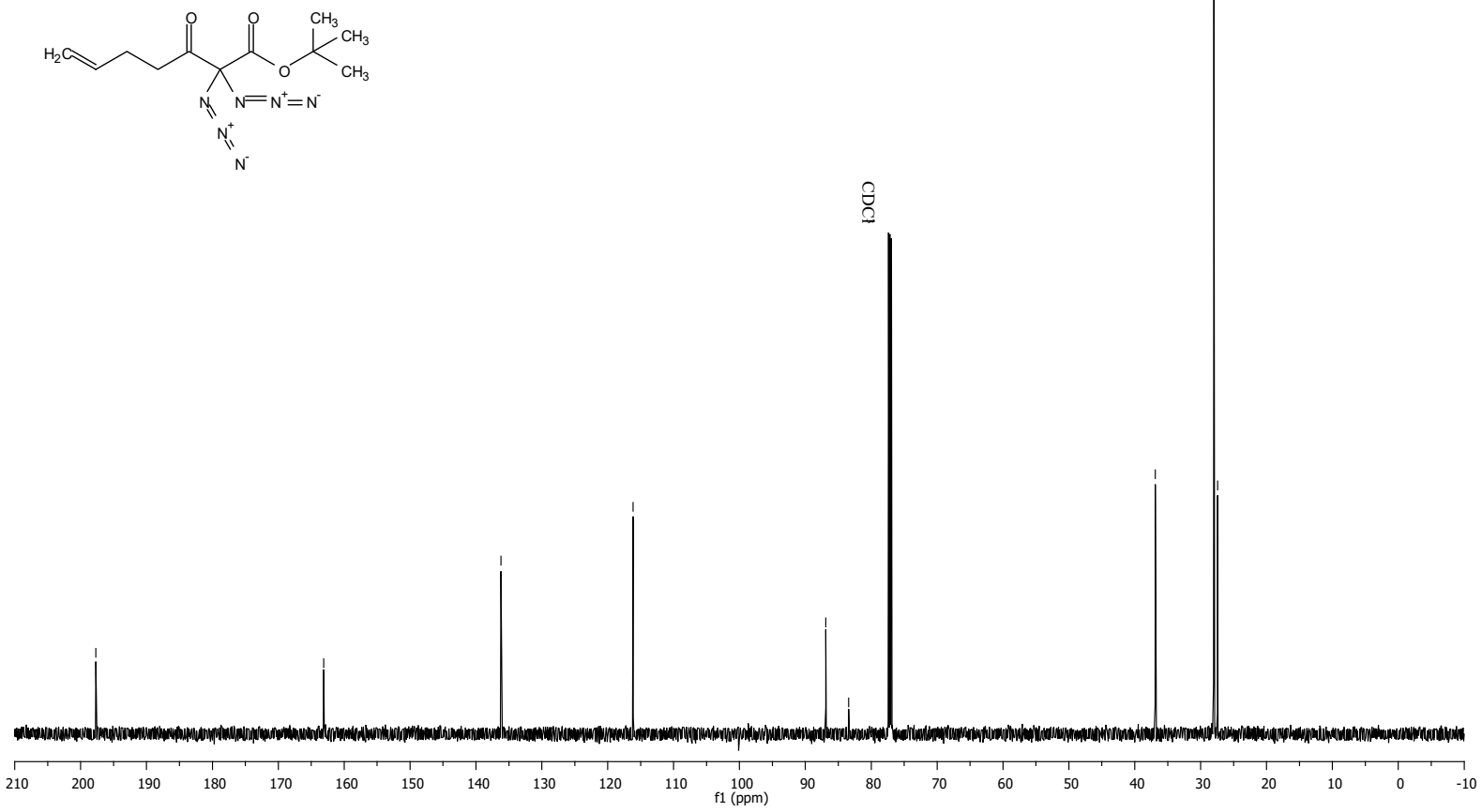


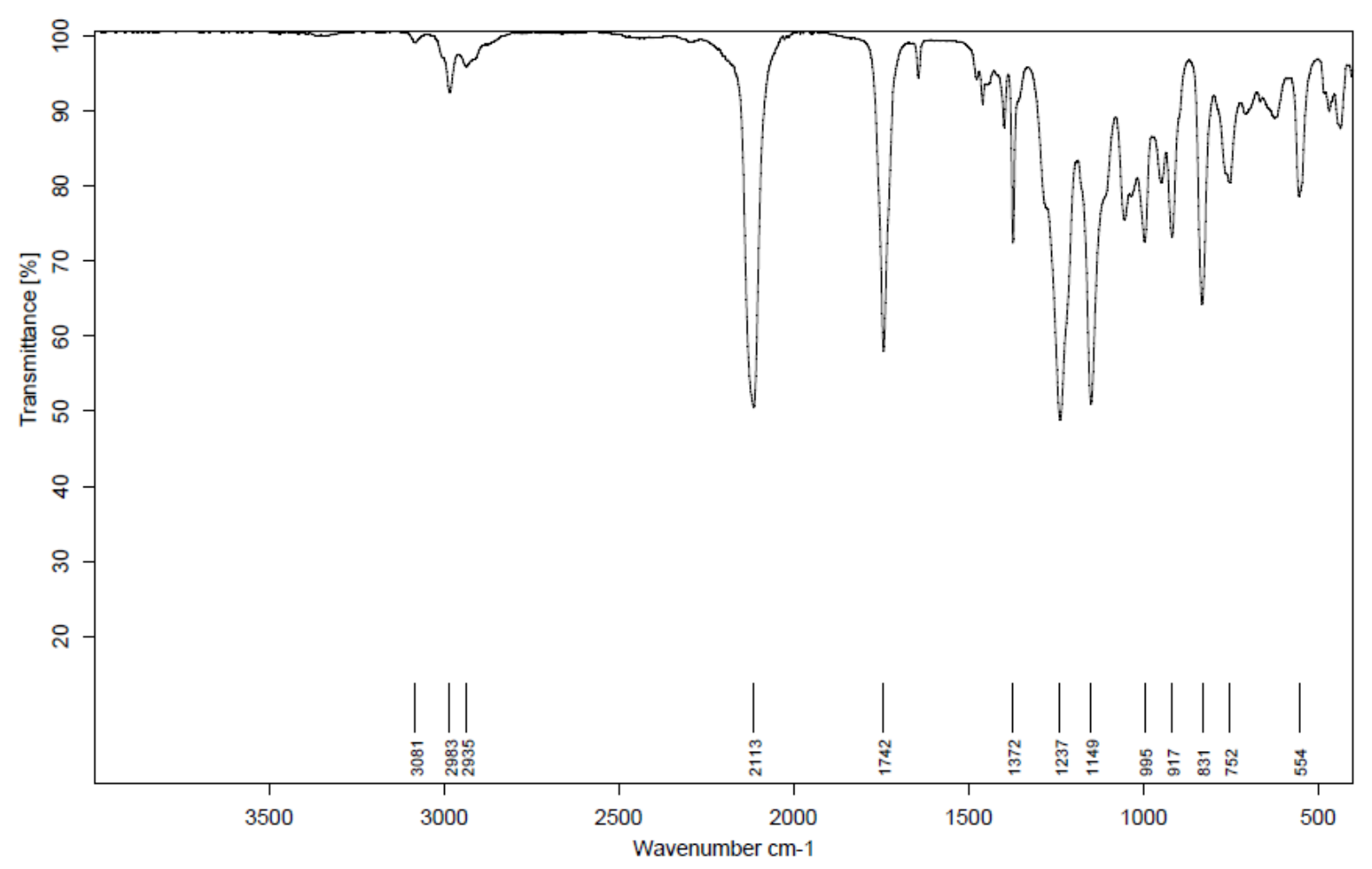

tert-Butyl 2,2-diazido-3-oxohept-6-enoate

'H NMR (400MHz, CDCl)

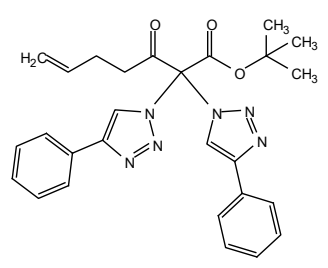

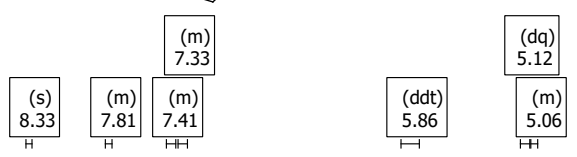
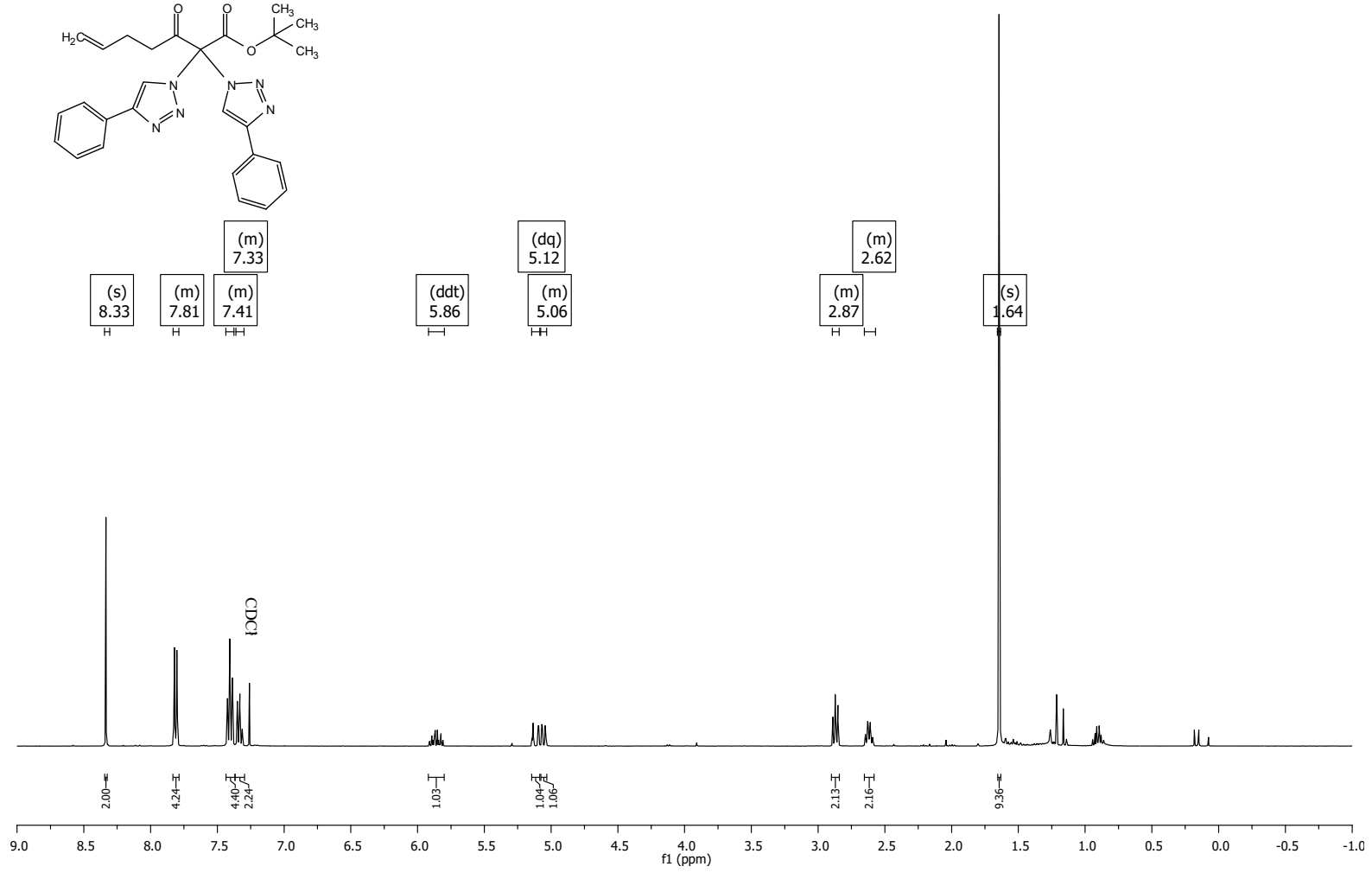

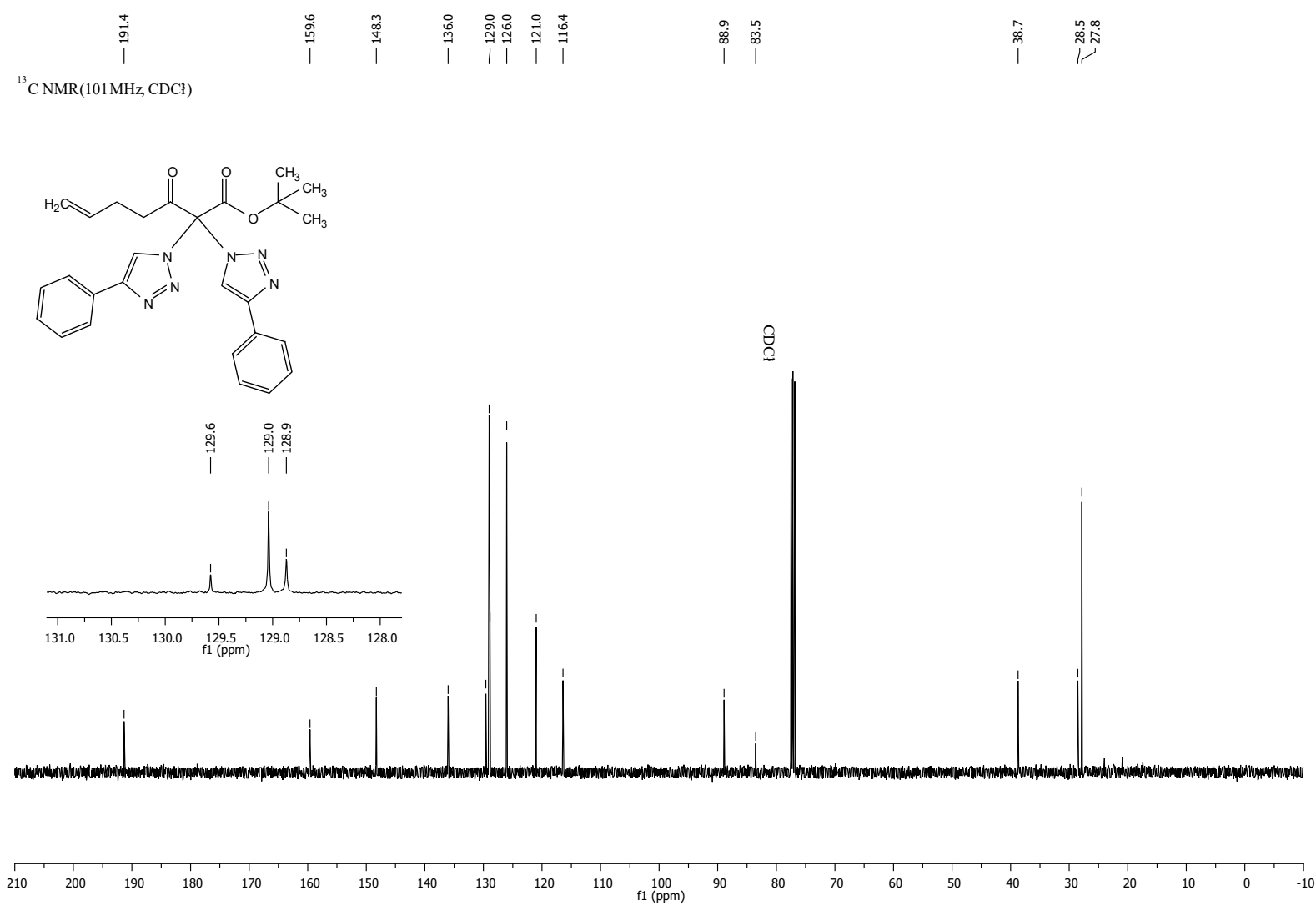

tert-Butyl 2,2-diazido-7-(benzyloxy)-3-oxoheptanoate (2t)

${ }^{1} \mathrm{H}$ NMR(400MHz, CDCl)

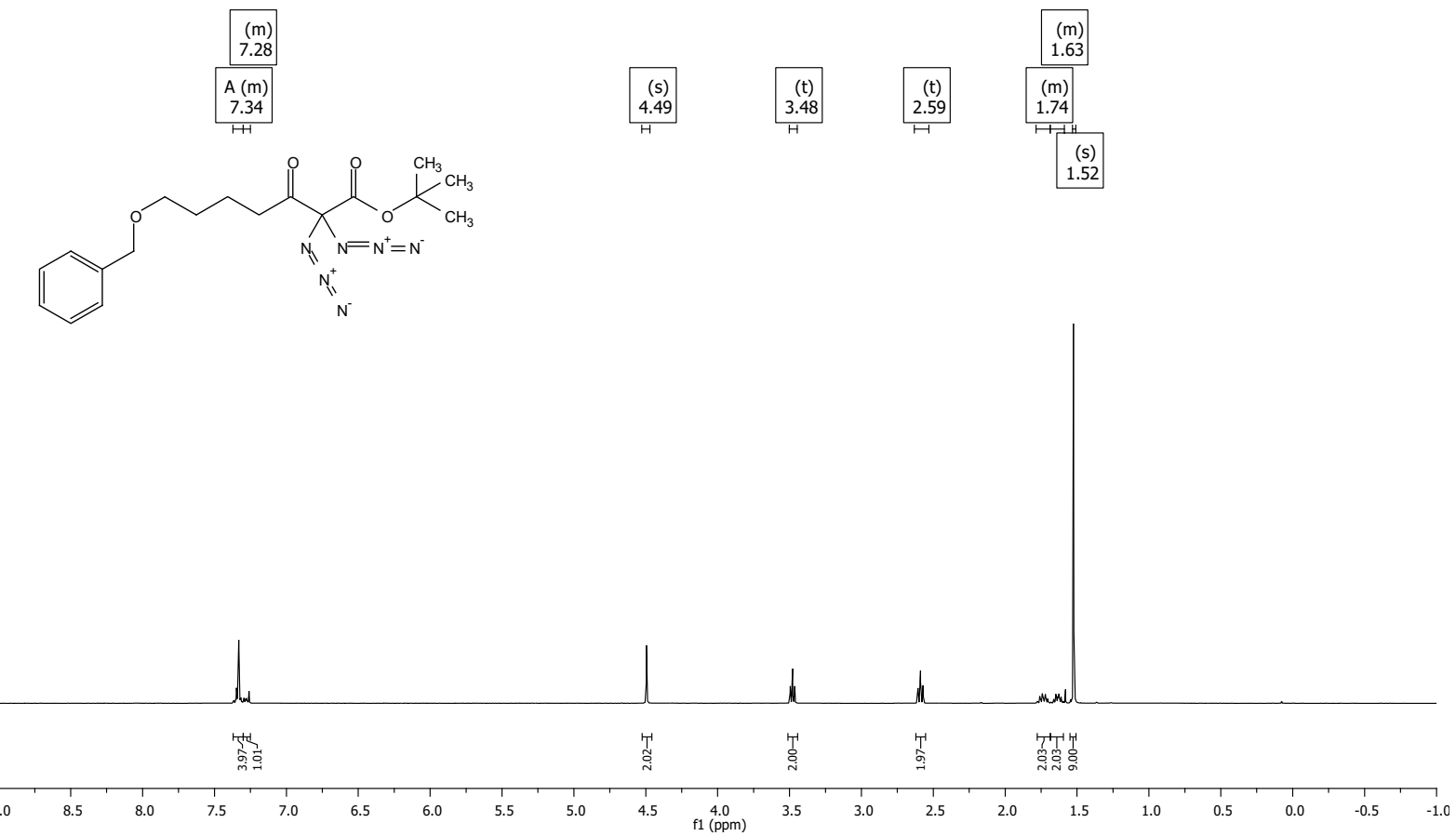




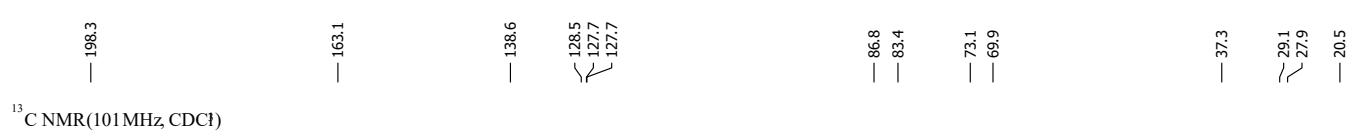<smiles></smiles>
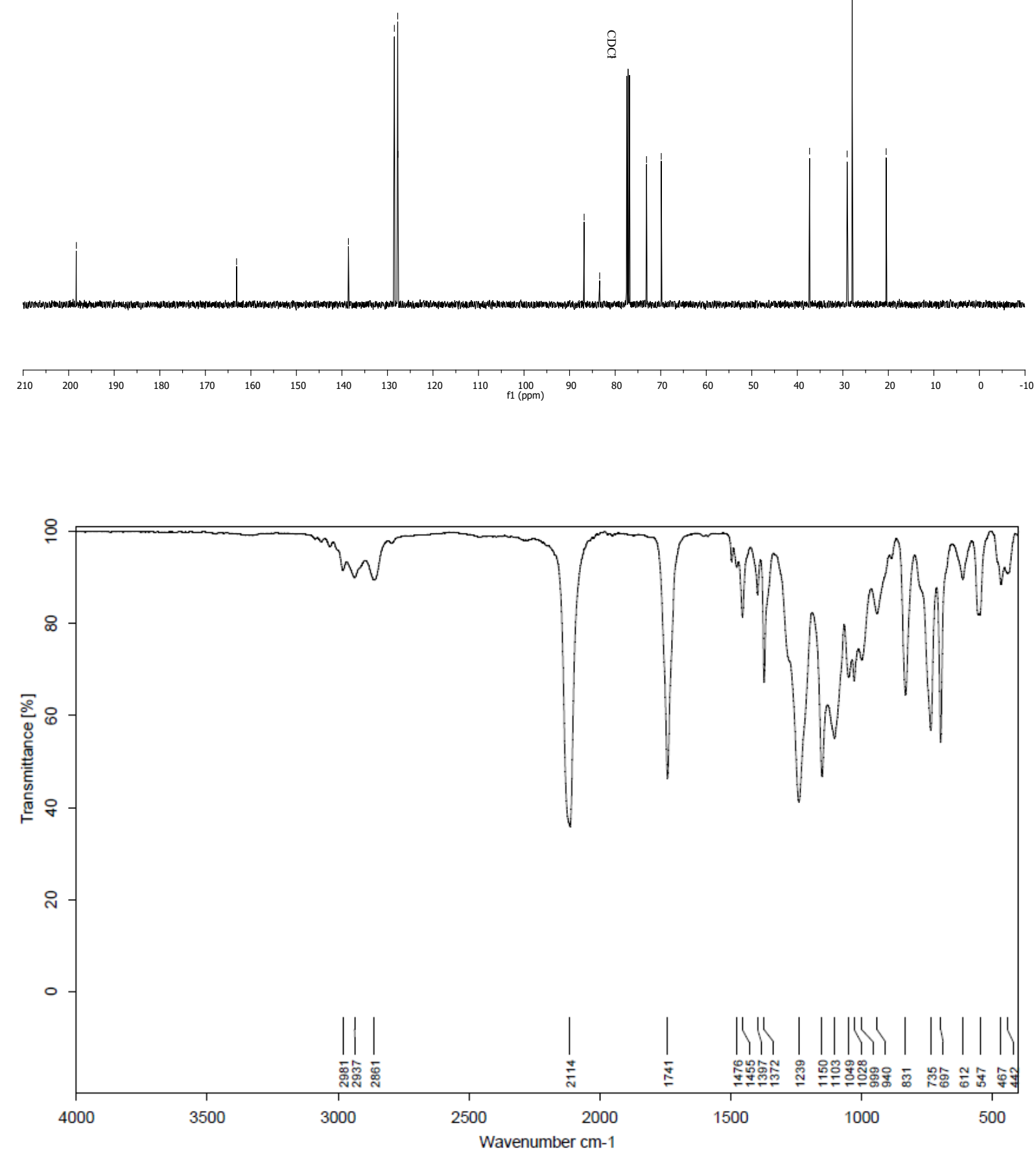
tert-Butyl 7-(benzyloxy)-2,2-bis(4,5,6,7,8,9-hexahydro-1H-cycloocta[d][1,2,3]triazol-1-yl)-3-oxoheptanoate ${ }^{1} \mathrm{H} \mathrm{NMR}(400 \mathrm{MHz}, \mathrm{CDCl})$

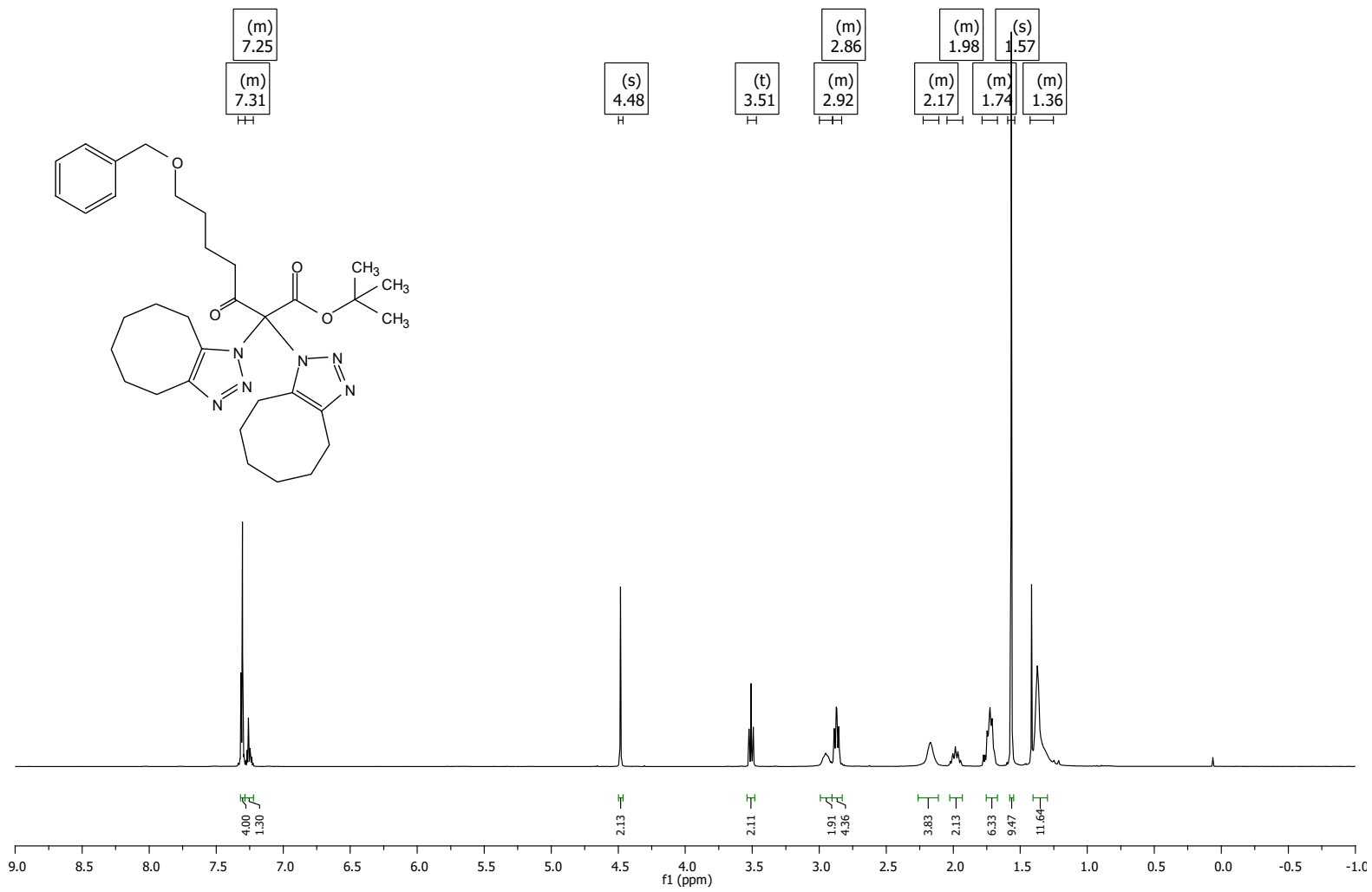

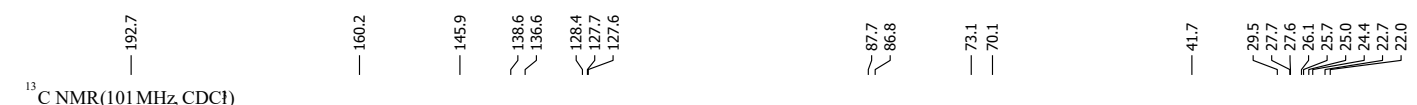
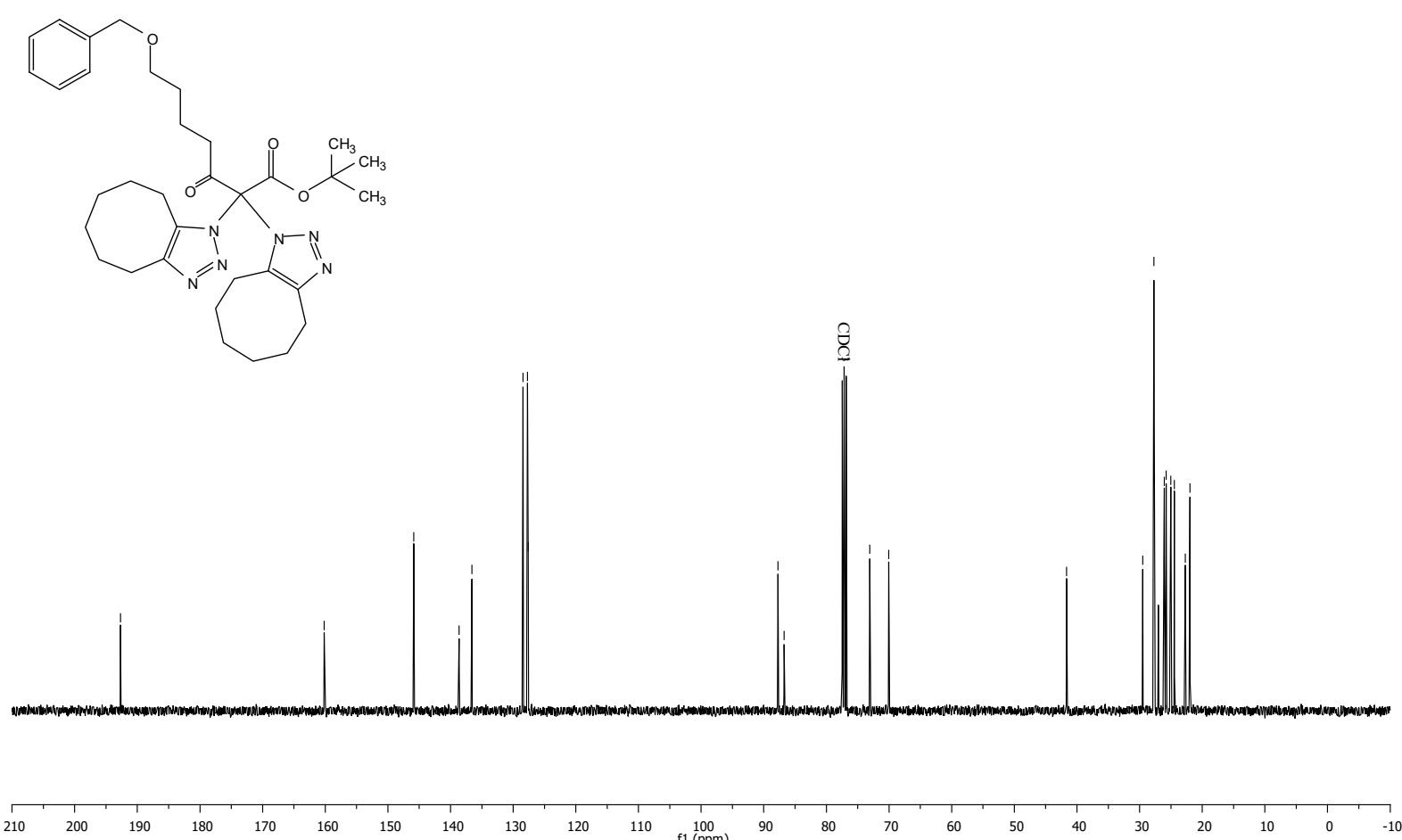
tert-Butyl 7-acetoxy-2,2-diazido-3-oxoheptanoate (2u)

${ }^{1} \mathrm{H}$ NMR(400 MHz, CDCl)<smiles>CC(=O)OCCCCC(=O)C(N=[N+]=[N-])(N=[N+]=[N-])C(=O)OC(C)(C)C</smiles>

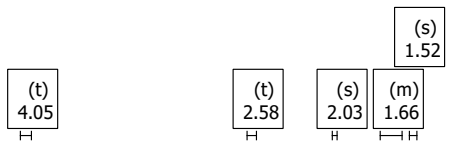

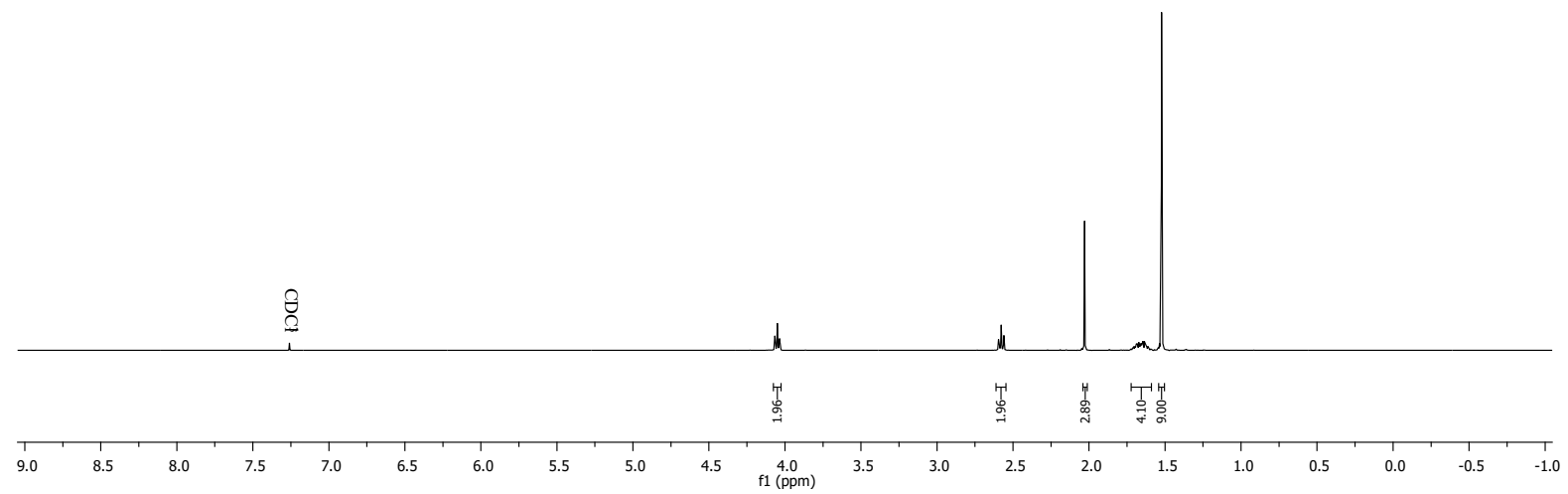

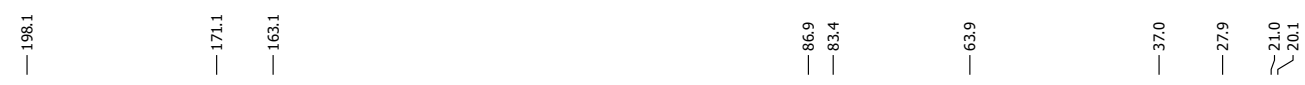

${ }^{13} \mathrm{C} \mathrm{NMR}(101 \mathrm{MHz}, \mathrm{CDCl})$<smiles>CC(=O)OCCCCC(=O)C(N=[N+]=[N-])(N=[N+]=[N-])C(=O)OC(C)(C)C</smiles>

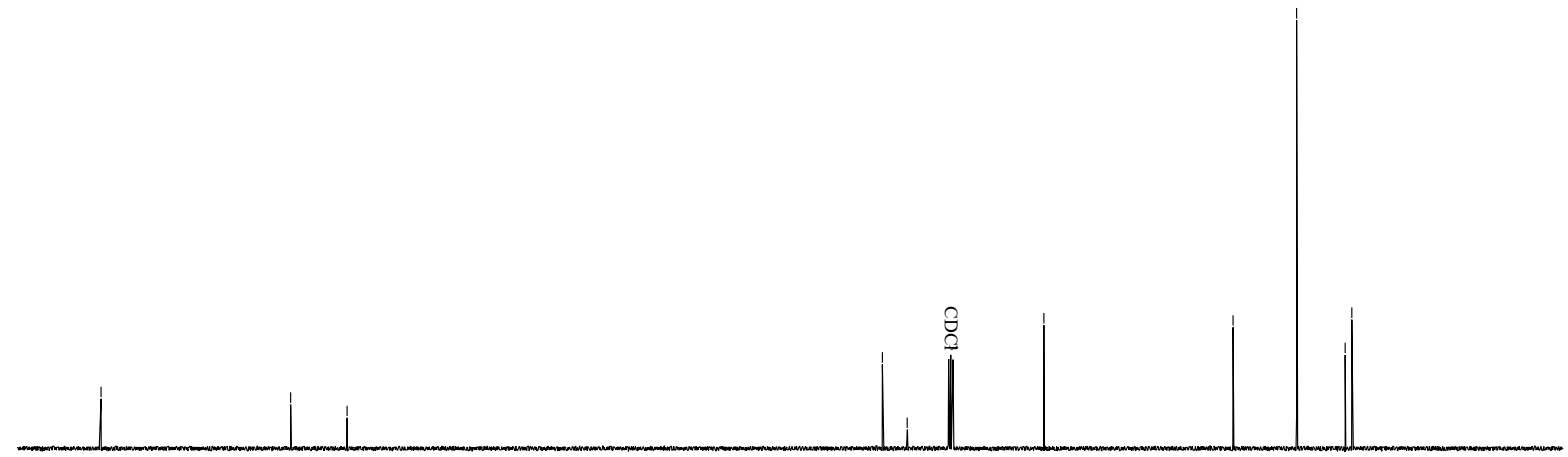




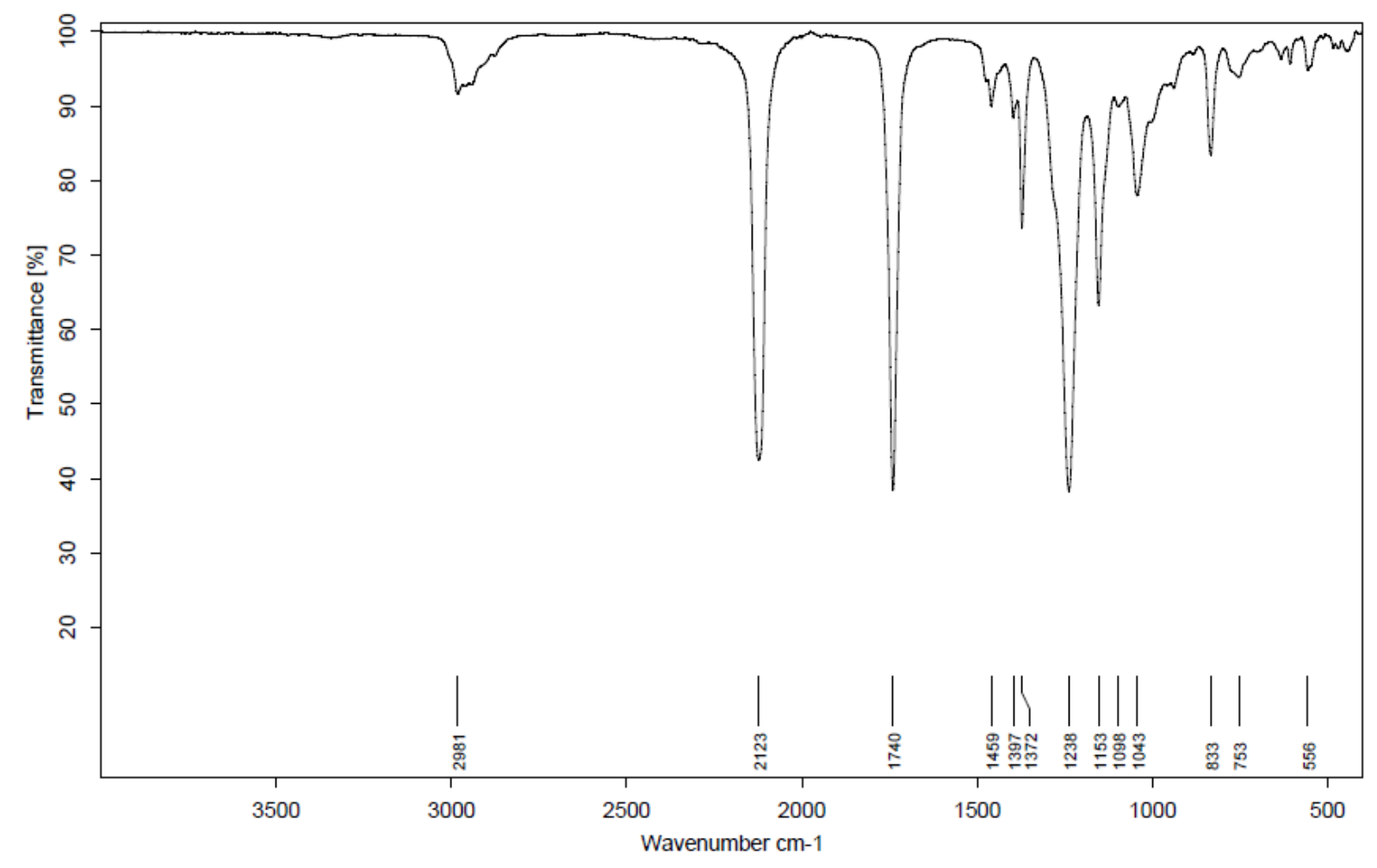

tert-Butyl 7-acetoxy-2,2-bis(4,5,6,7,8,9-hexahydro-1H-cycloocta[d] [1,2,3]triazol-1-yl)-3oxoheptanoate

${ }^{1}$ H NMR(400MHz, CDC1)
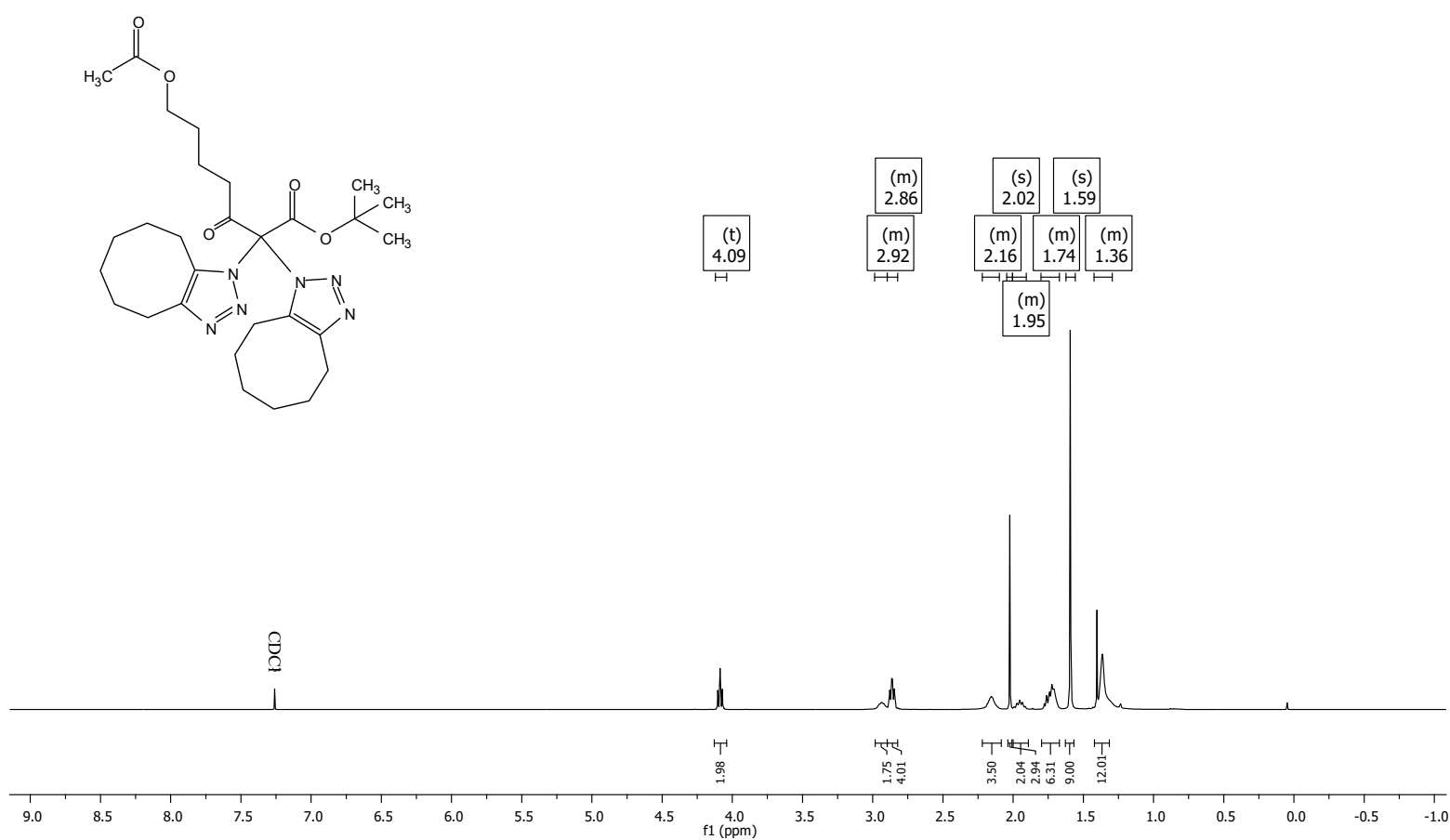

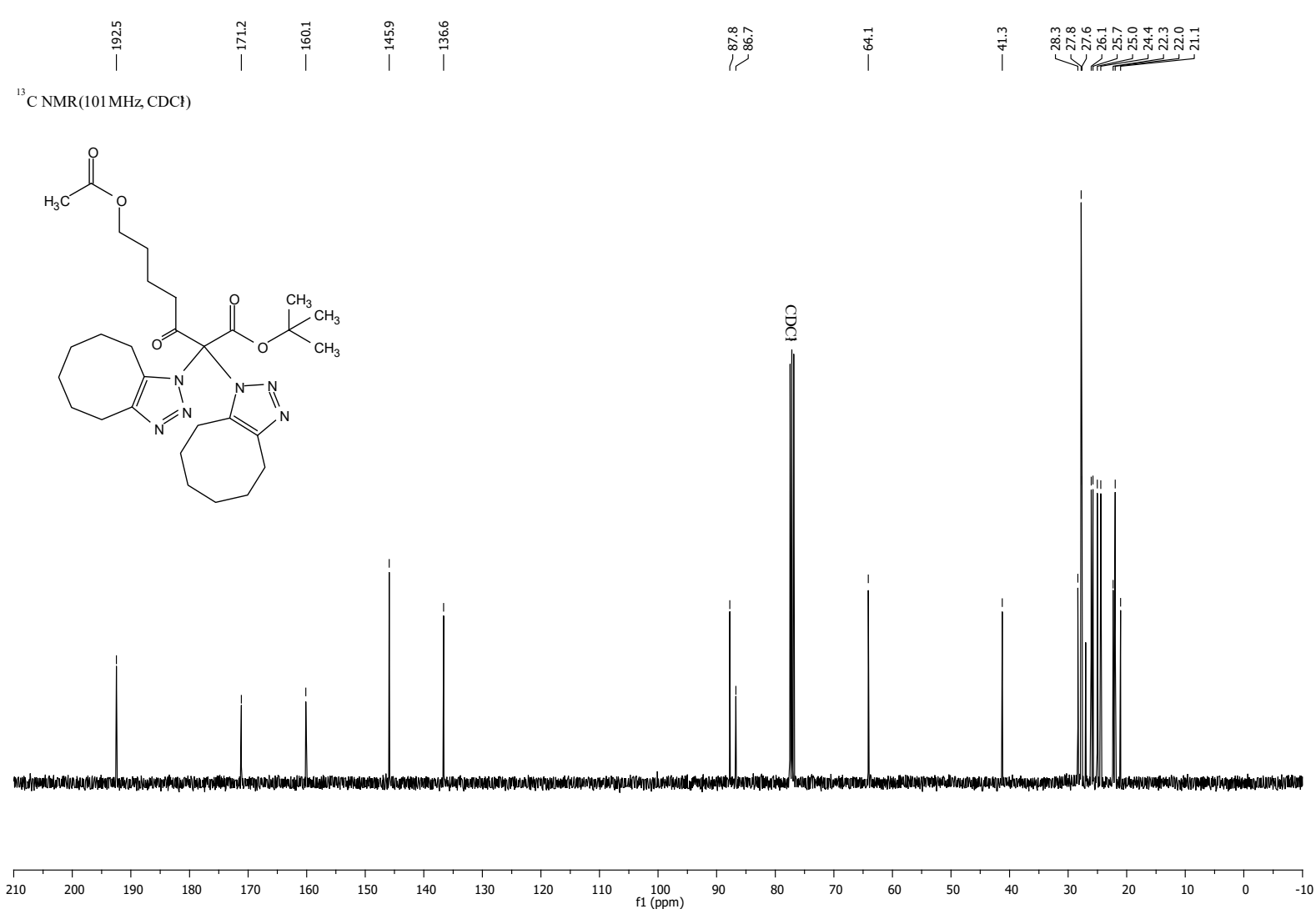

tert-Butyl 2,2-diazido-6-((tert-butyldimethylsilyl)oxy)-3-oxohexanoate (2v)

${ }^{1} \mathrm{H} \mathrm{NMR}(400 \mathrm{MHz}, \mathrm{CDCl})$
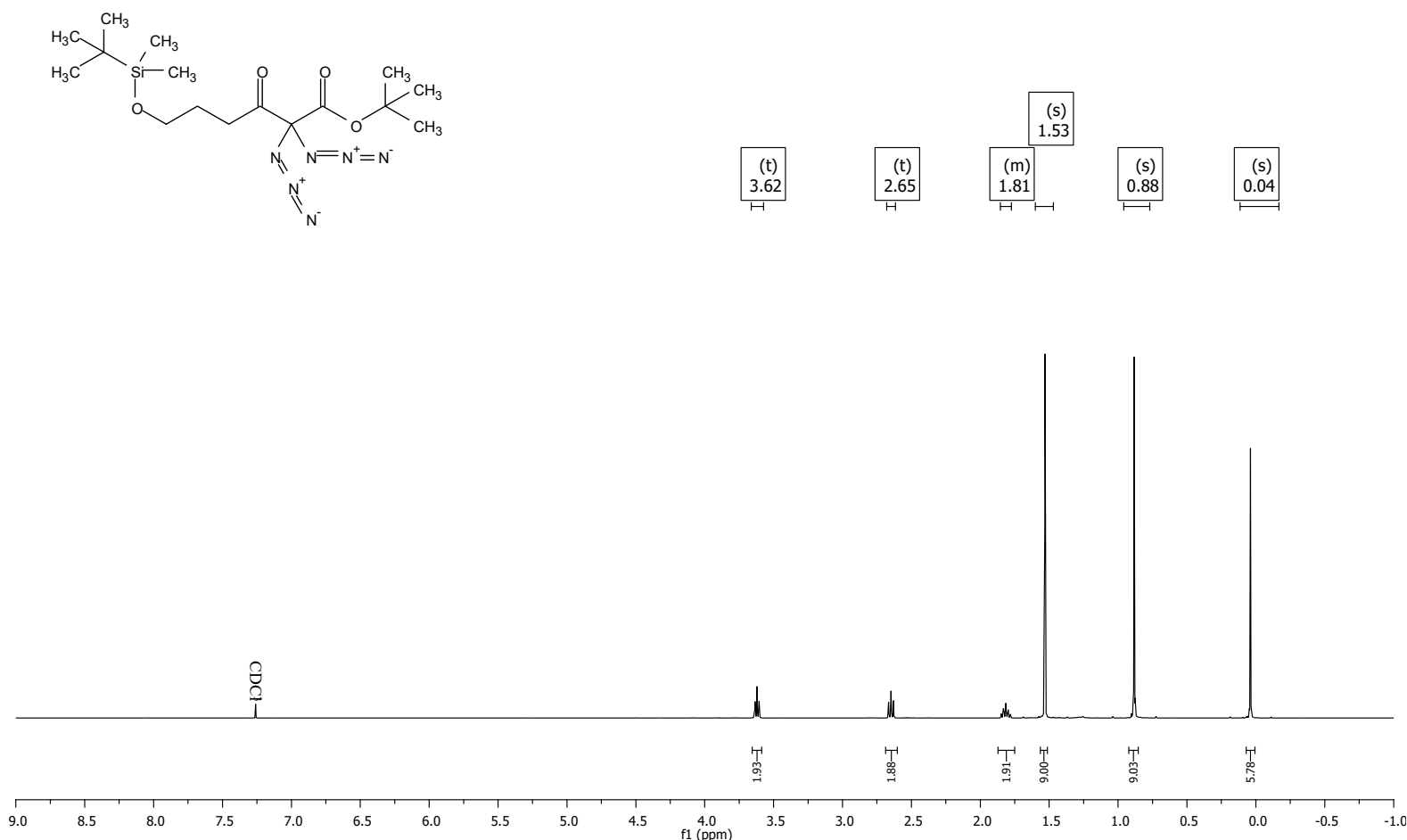


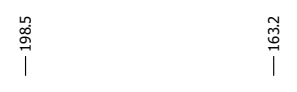

这

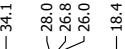

${ }^{13} \mathrm{C} \mathrm{NMR}(101 \mathrm{MHz}, \mathrm{CDC})$<smiles>CC(C)(C)OC(=O)C1(C(=O)CCCOP(C)(C)(C)C(C)(C)C)NC(N)=NC1=NN</smiles>
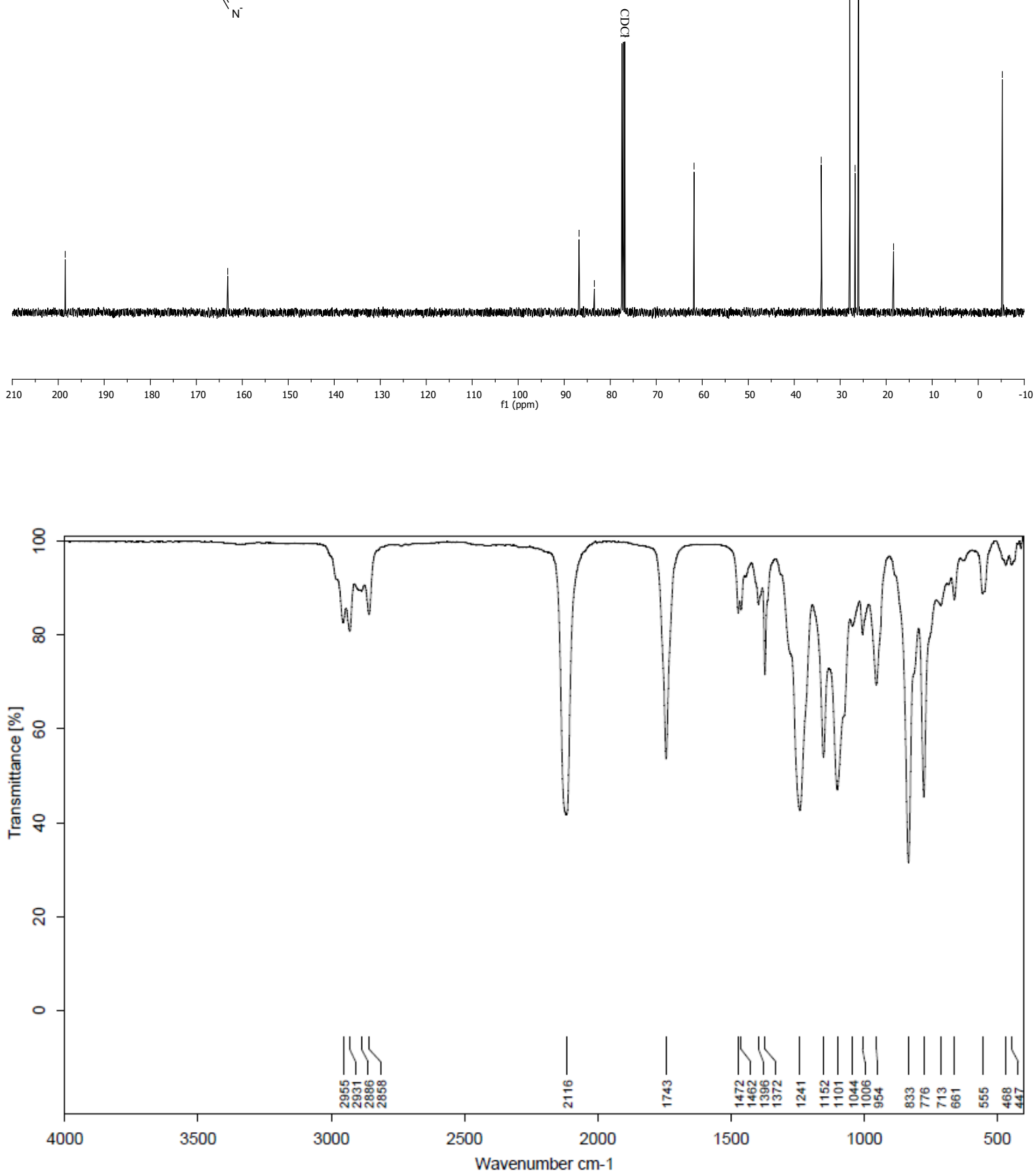
tert-Butyl 6-((tert-butyldimethylsilyl)oxy)-2,2-bis(4,5,6,7,8,9-hexahydro-1 $H$-cycloocta $[d][1,2,3]$ triazol-1yl)-3-oxohexanoate

${ }^{1} \mathrm{H}$ NMR(400MHz, CDCl)

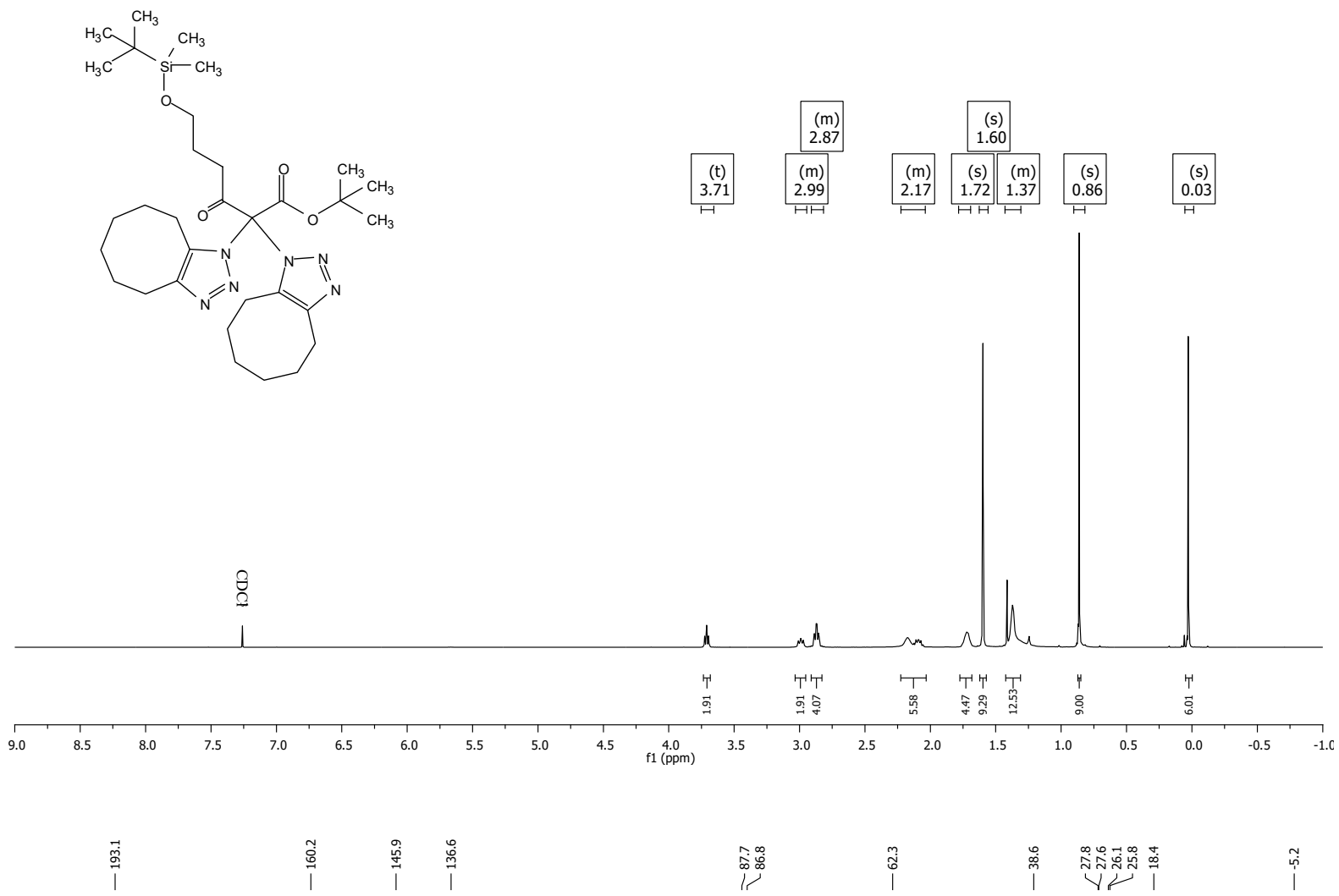

${ }^{13} \mathrm{C}$ NMR(101 MHz, CDC)
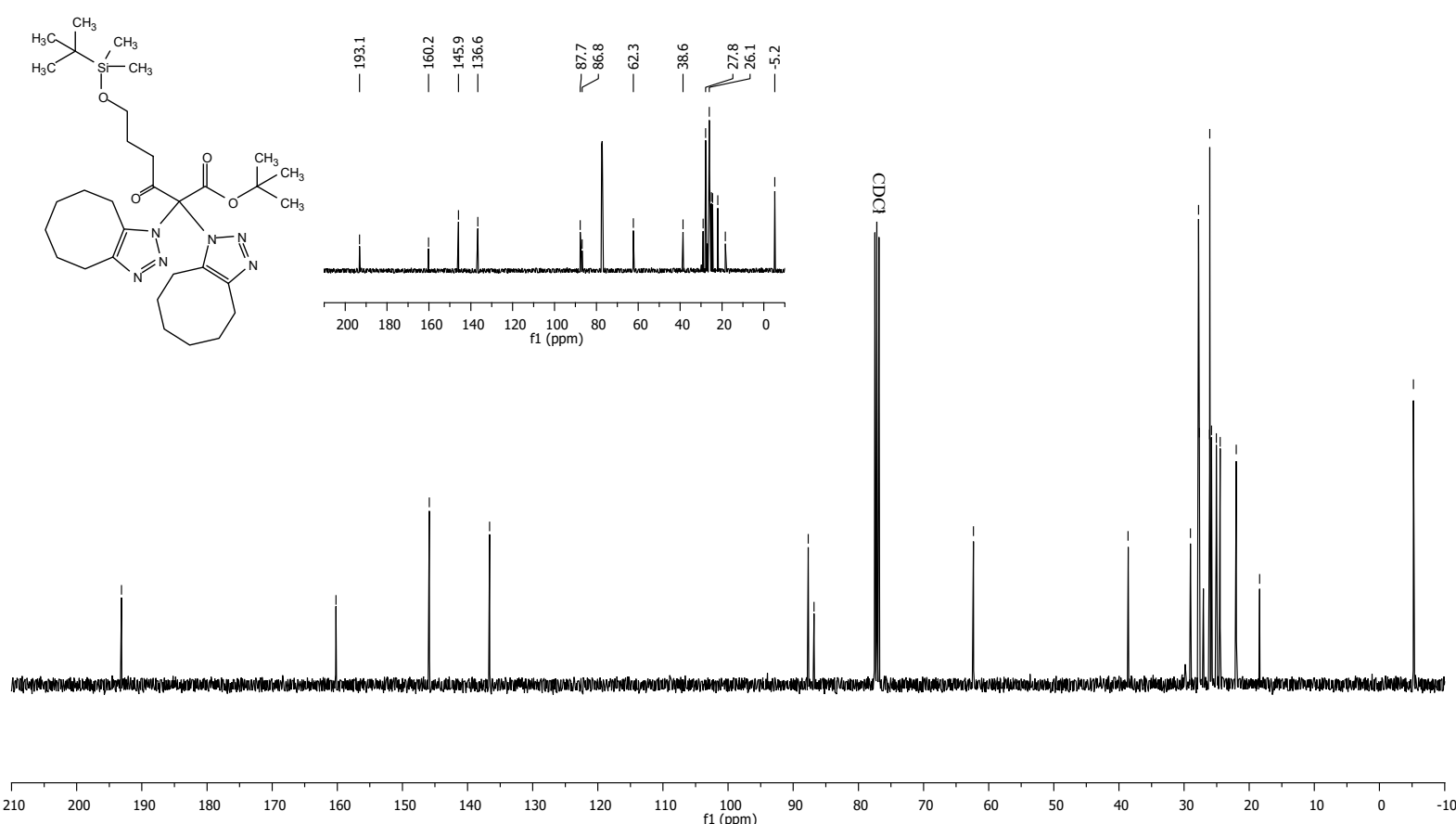
tert-Butyl 2,2,6-triazido-3-oxohexanoate (2w)

${ }^{1} \mathrm{H} \mathrm{NMR}(600 \mathrm{MHz}, \mathrm{CDCl})$
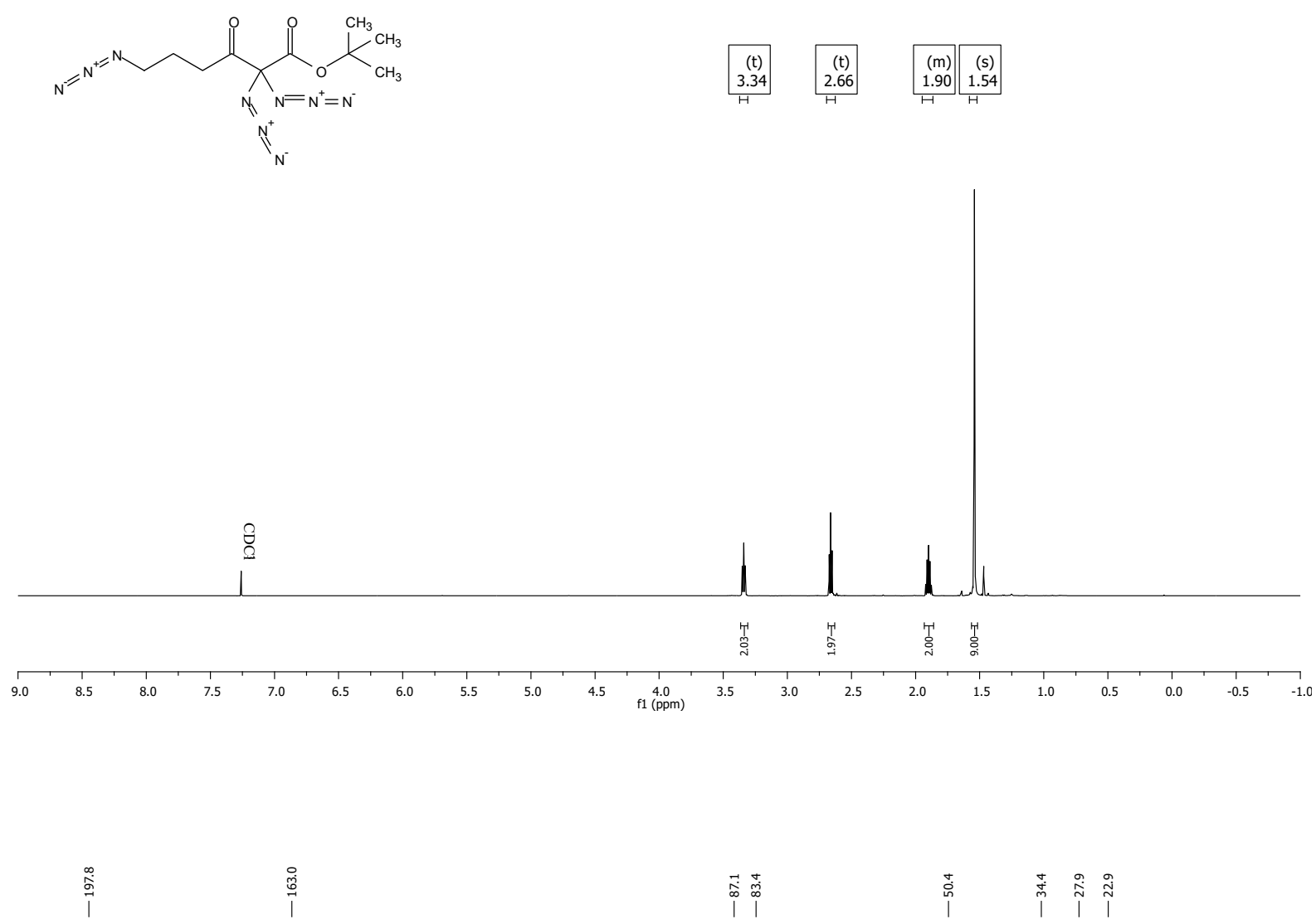

${ }^{13} \mathrm{C}$ NMR(151 MHz, CDCF)
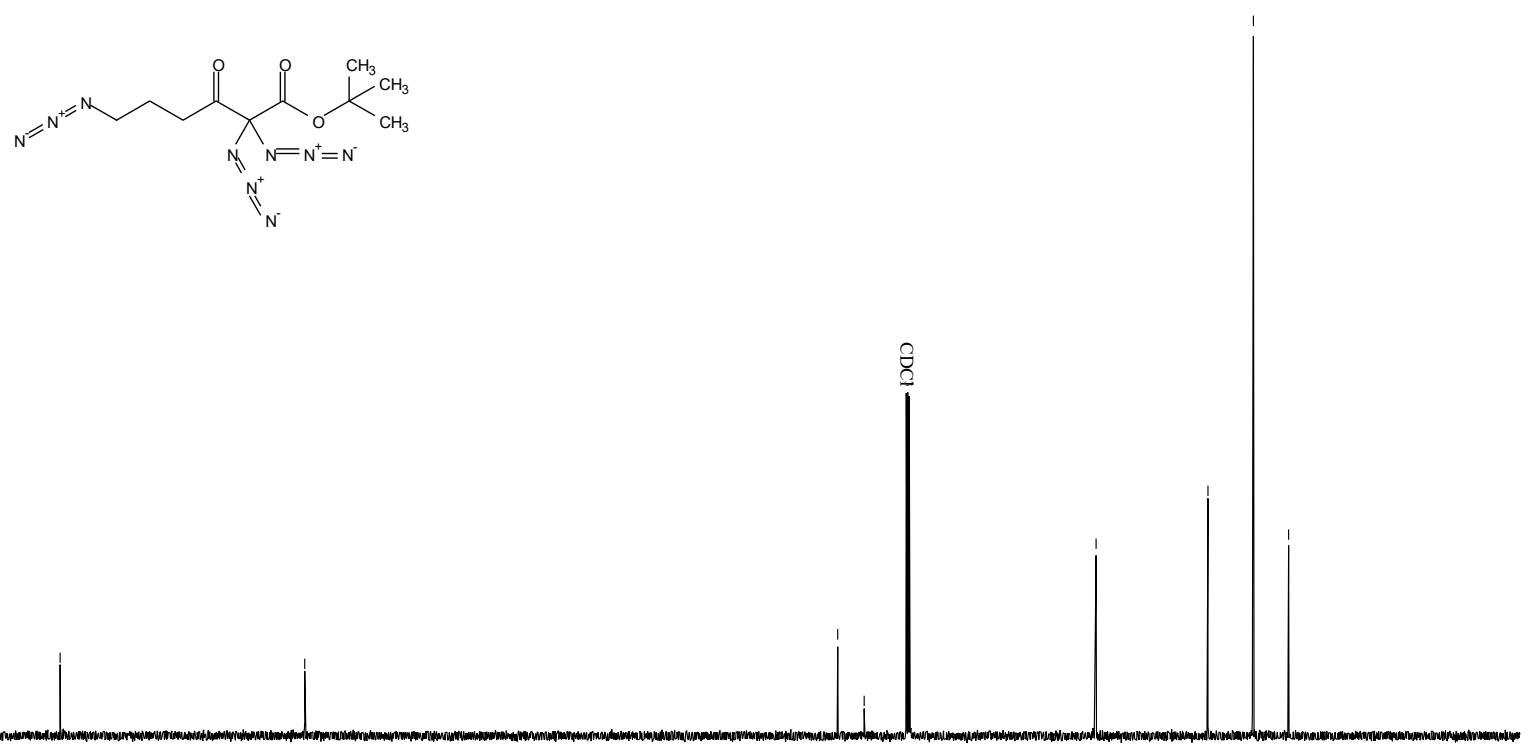

210

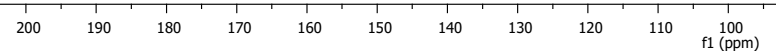

f1 100 


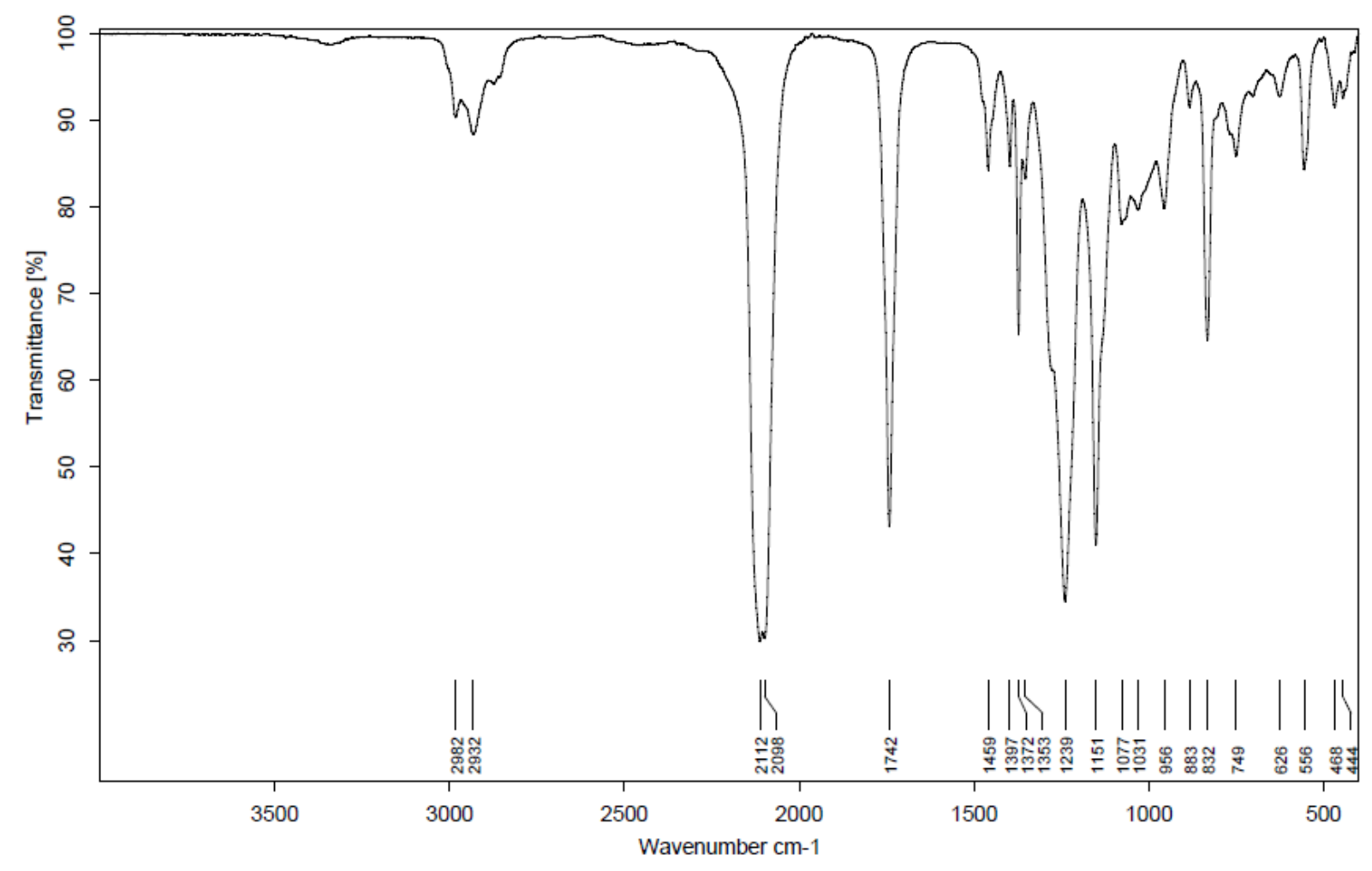

tert-Butyl 2,2,6-tris $(4,5,6,7,8,9-h e x a h y d r o-1 H$-cycloocta $[d][1,2,3]$ triazol-1-yl)-3-oxohexanoate

'H NMR(400MHz, CDCl)
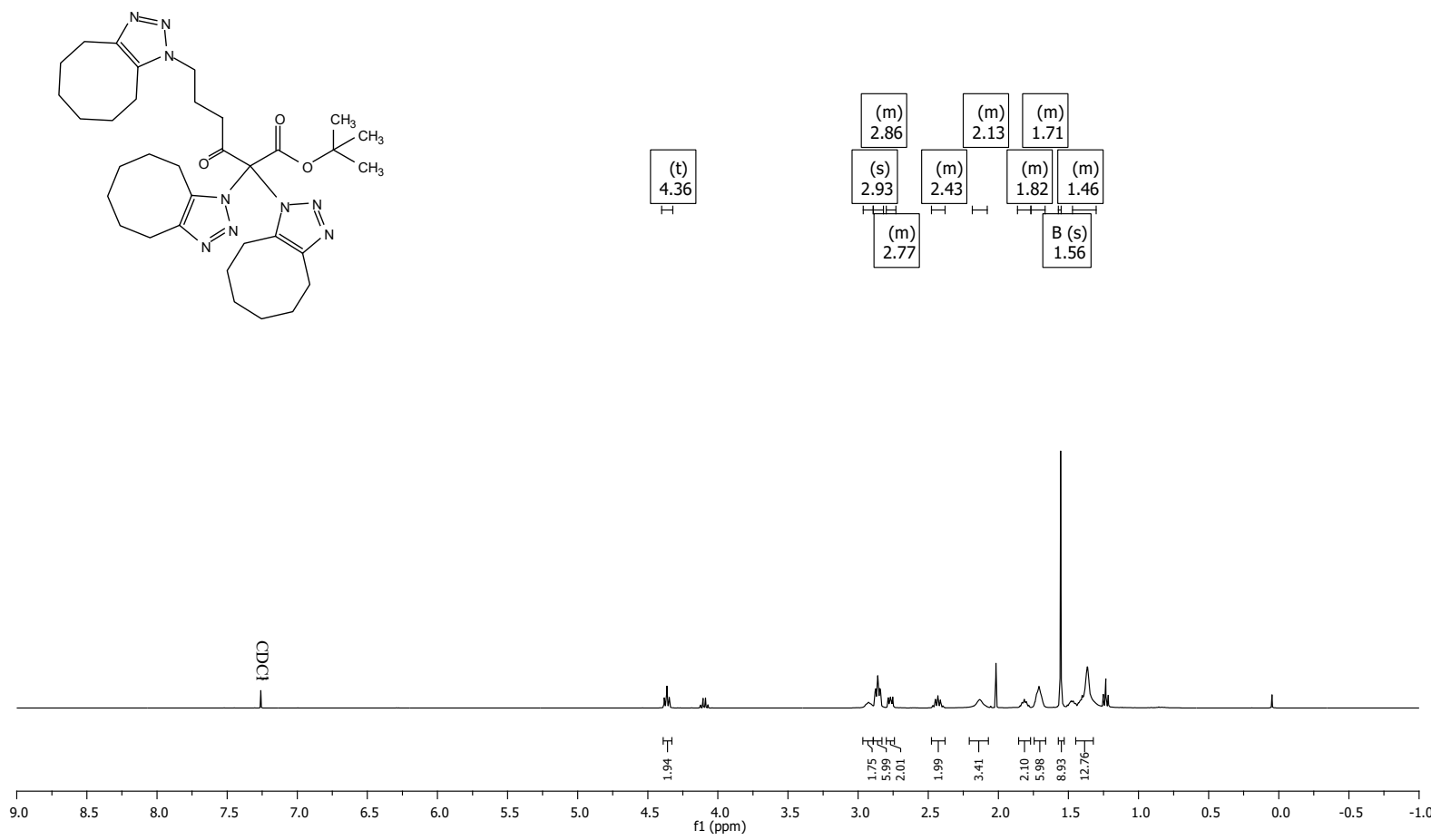


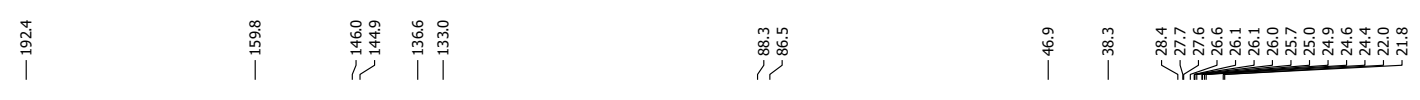

${ }^{13} \mathrm{C} \mathrm{NMR}(101 \mathrm{MHz}, \mathrm{CDCl})$

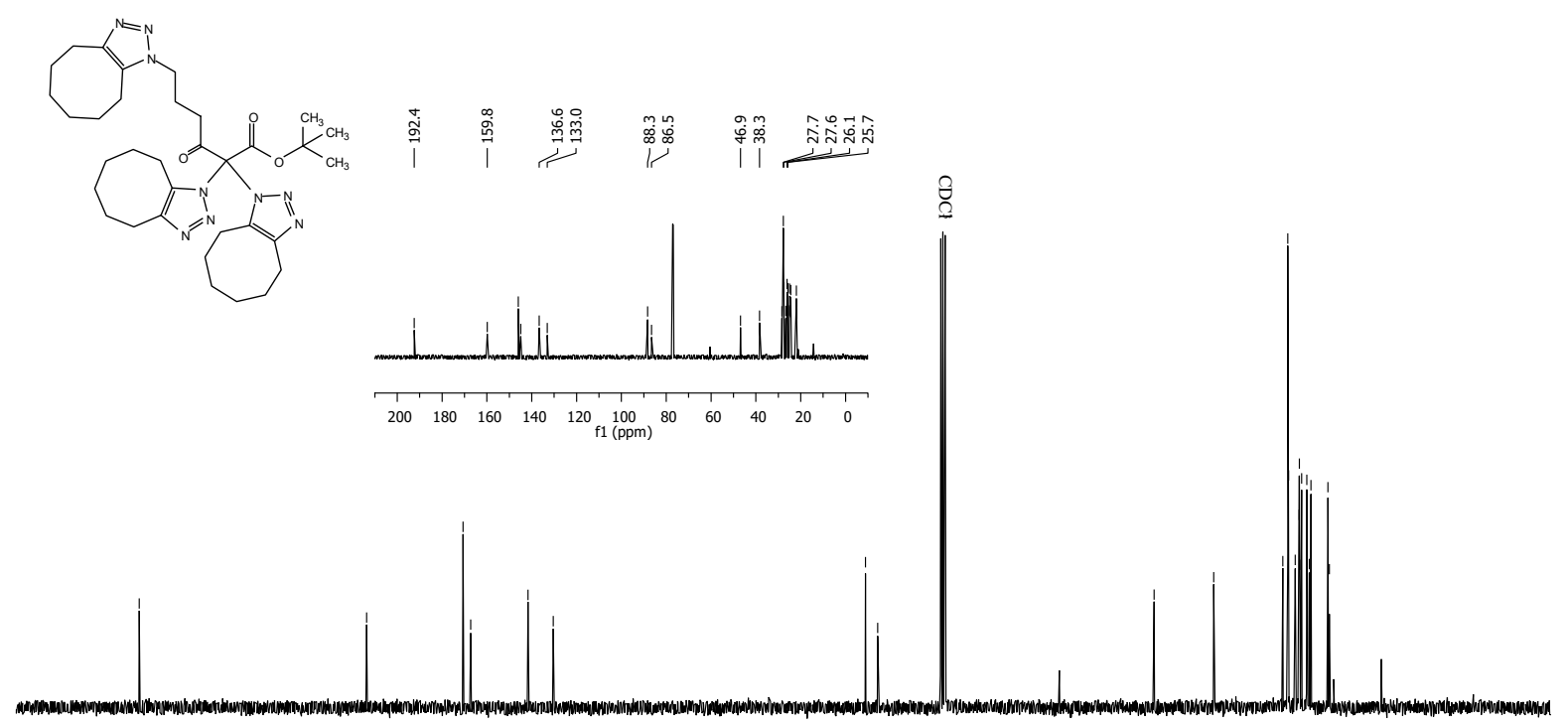

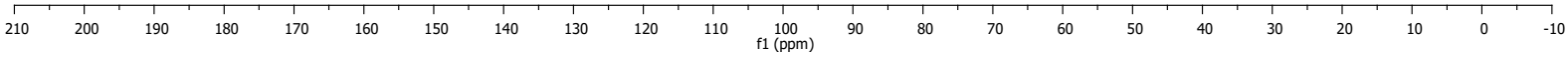

tert-Butyl 2,2-diazido-5-hydroxy-5-methyl-3-oxohexanoate (2x)

${ }^{1} \mathrm{H}$ NMR $(400 \mathrm{MHz}, \mathrm{CDCl})$

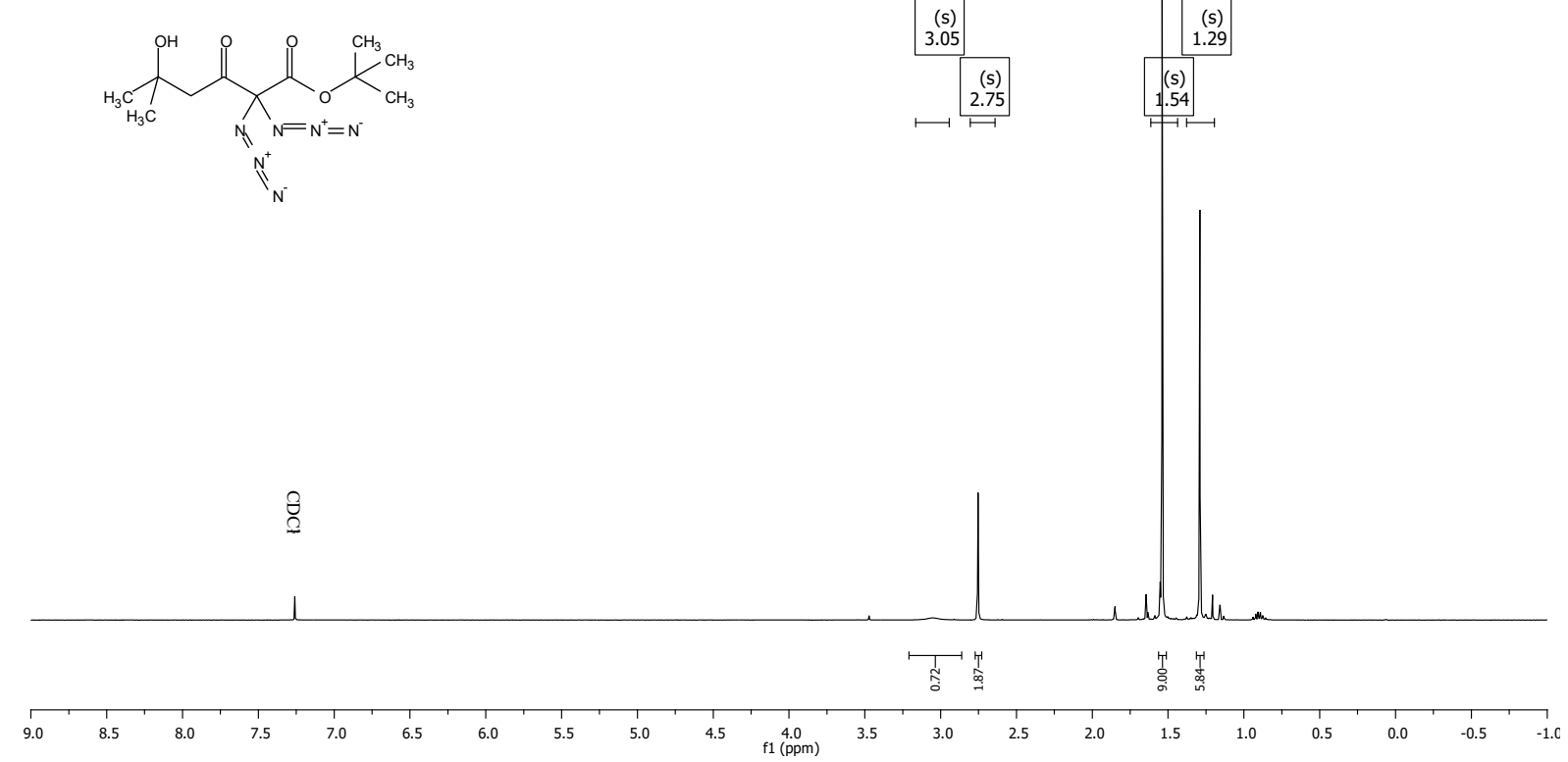



$\stackrel{\circ}{\stackrel{\circ}{i}}$

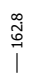

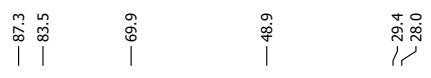

${ }^{13} \mathrm{C}$ NMR(151 MHz, CDCl)
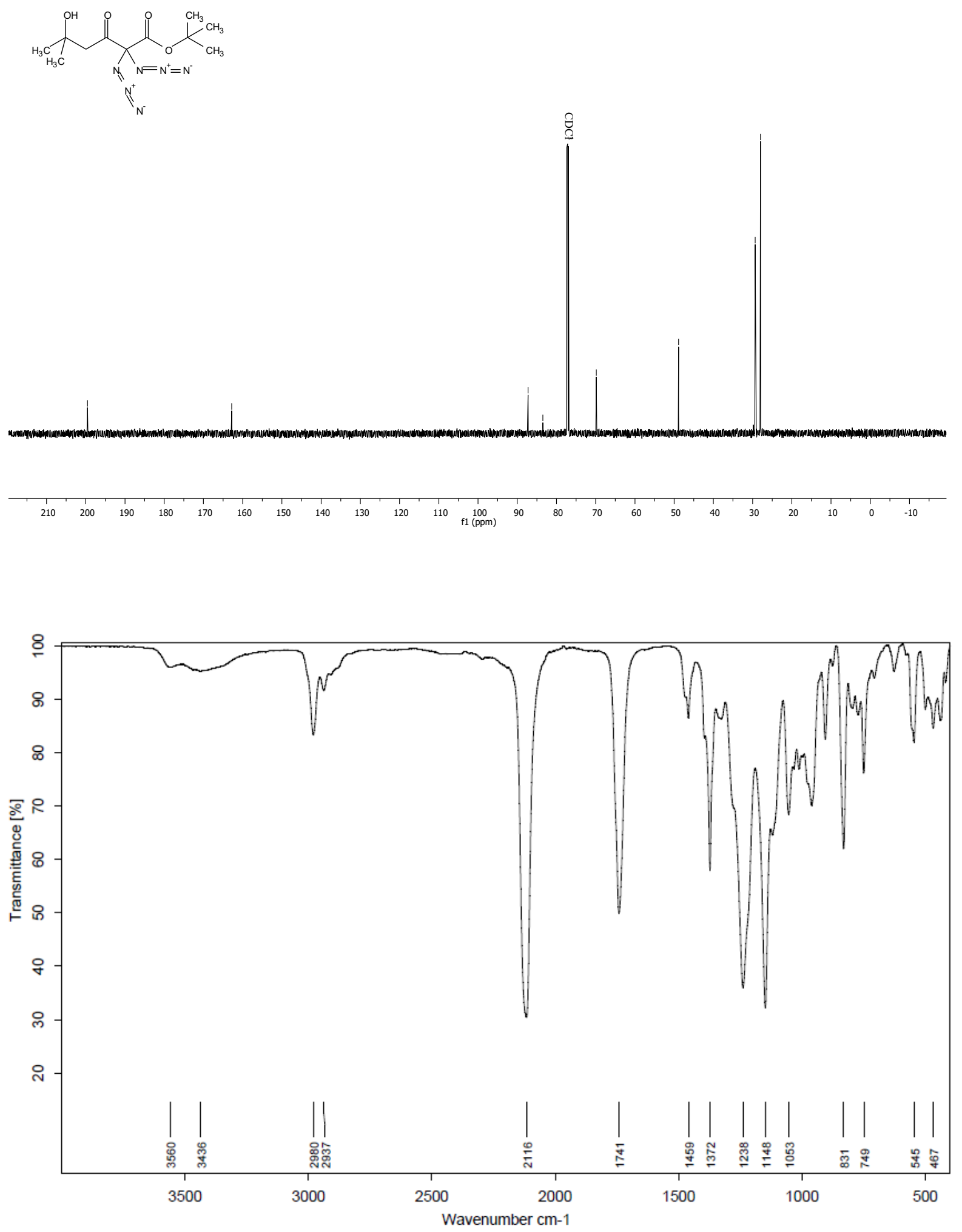
tert-Butyl 2,2-diazido-5-hydroxy-6-methyl-3-oxoheptanoate (2y)

${ }^{1} \mathrm{H} \mathrm{NMR}(400 \mathrm{MHz}, \mathrm{CDCl})$
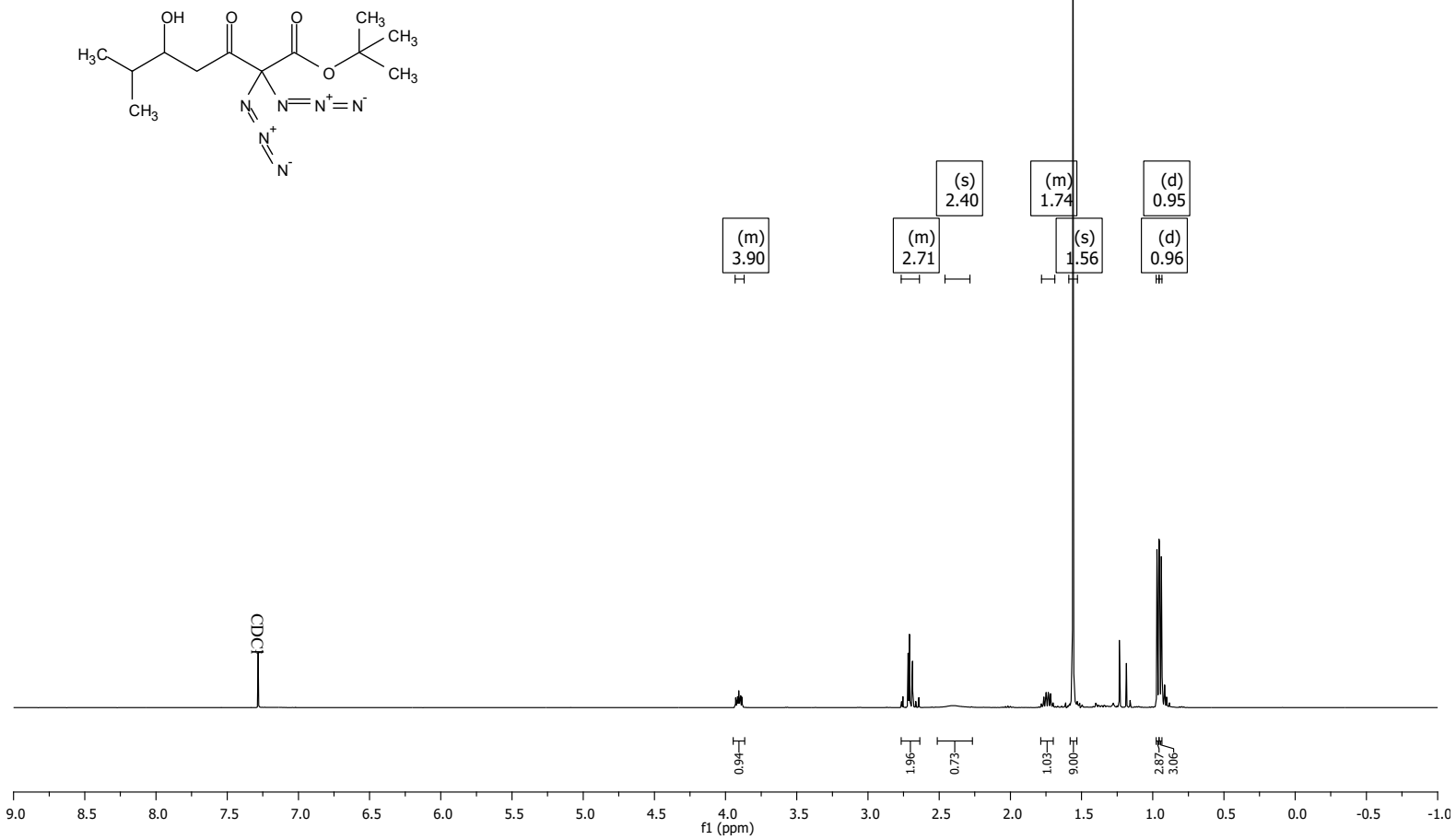

${ }^{13} \mathrm{C} \mathrm{NMR}(101 \mathrm{MHz}, \mathrm{CDCl})$
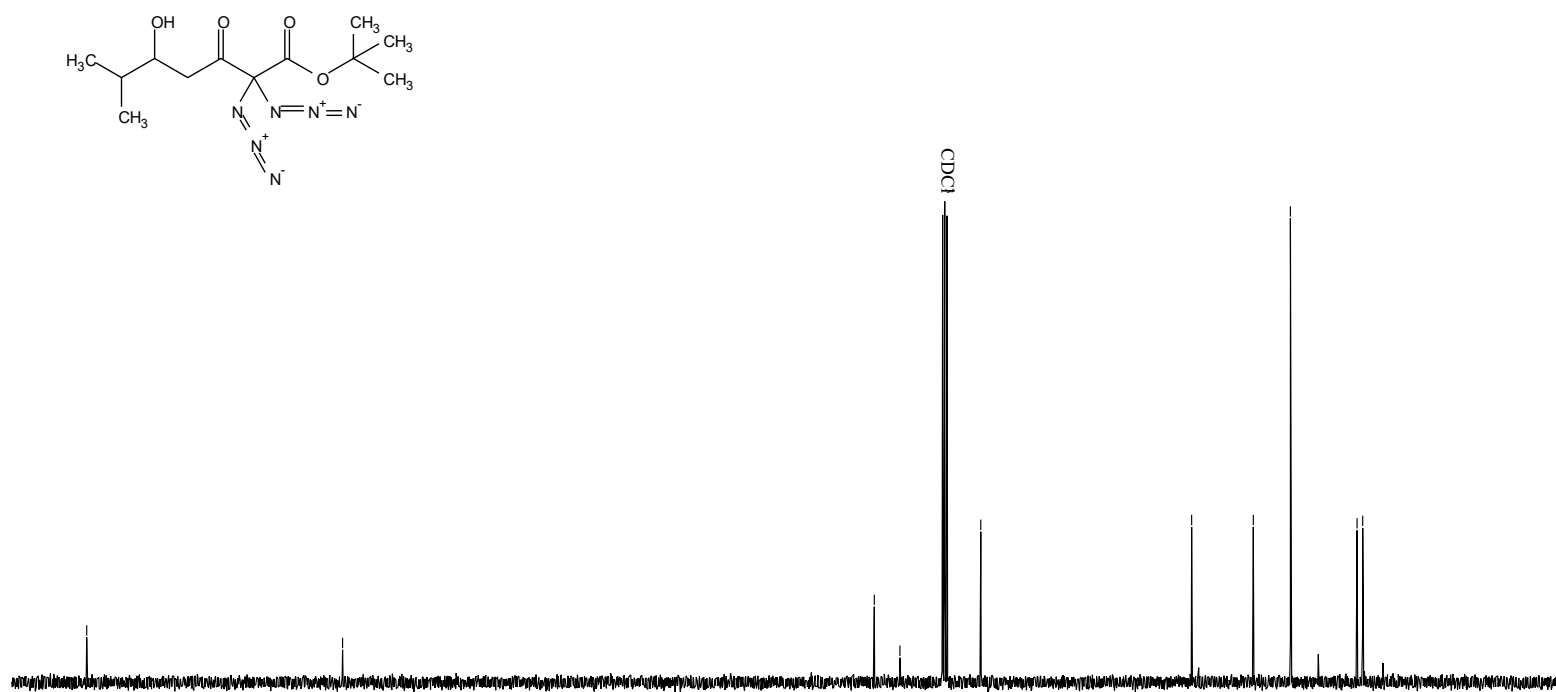


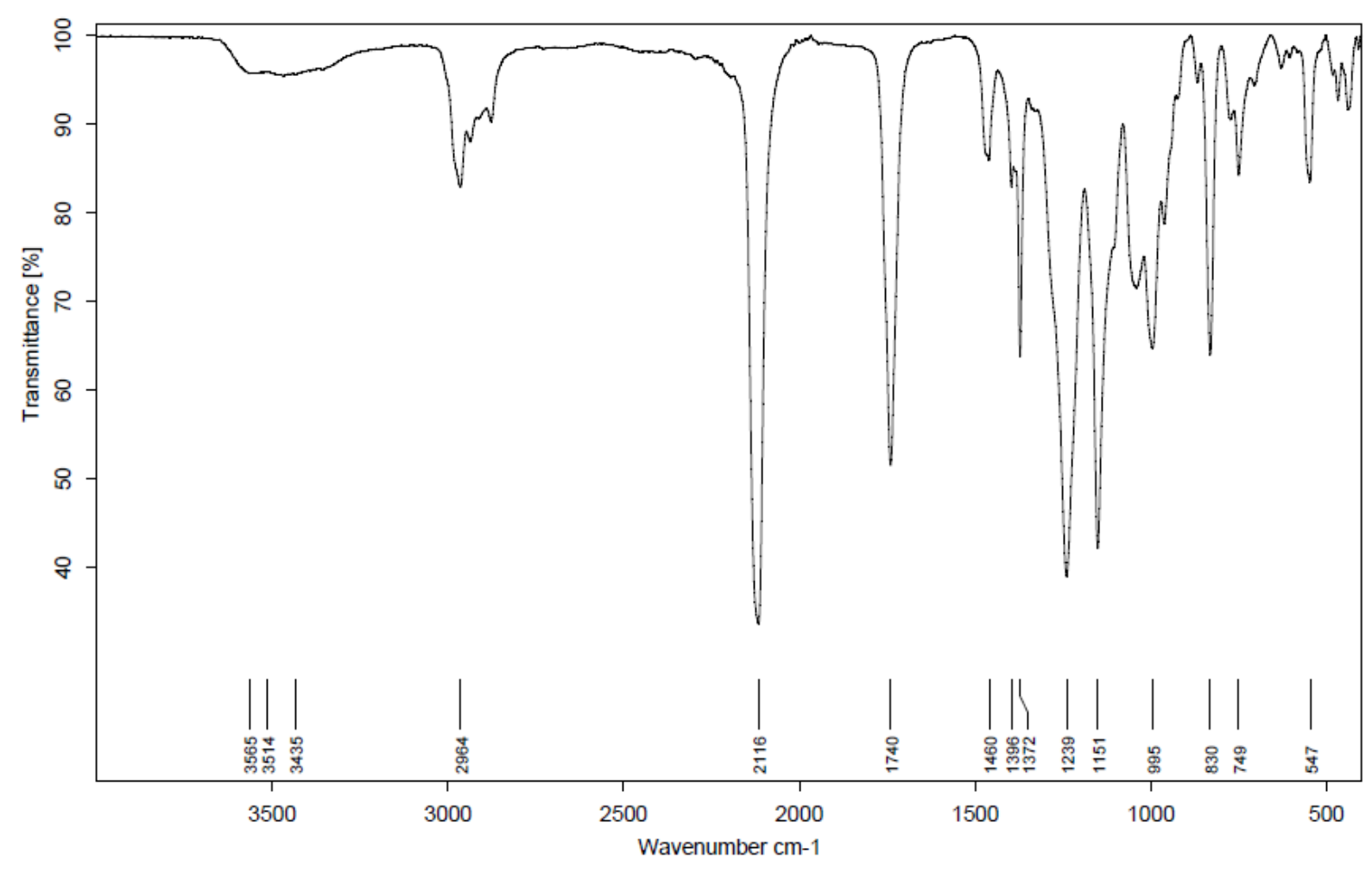

tert-Butyl 2,2-bis(4,5,6,7,8,9-hexahydro-1H-cycloocta[d] [1,2,3]triazol-1-yl)-5-hydroxy-6-methyl-3-oxoheptanoate

'H NMR(400MHz, CDCl)
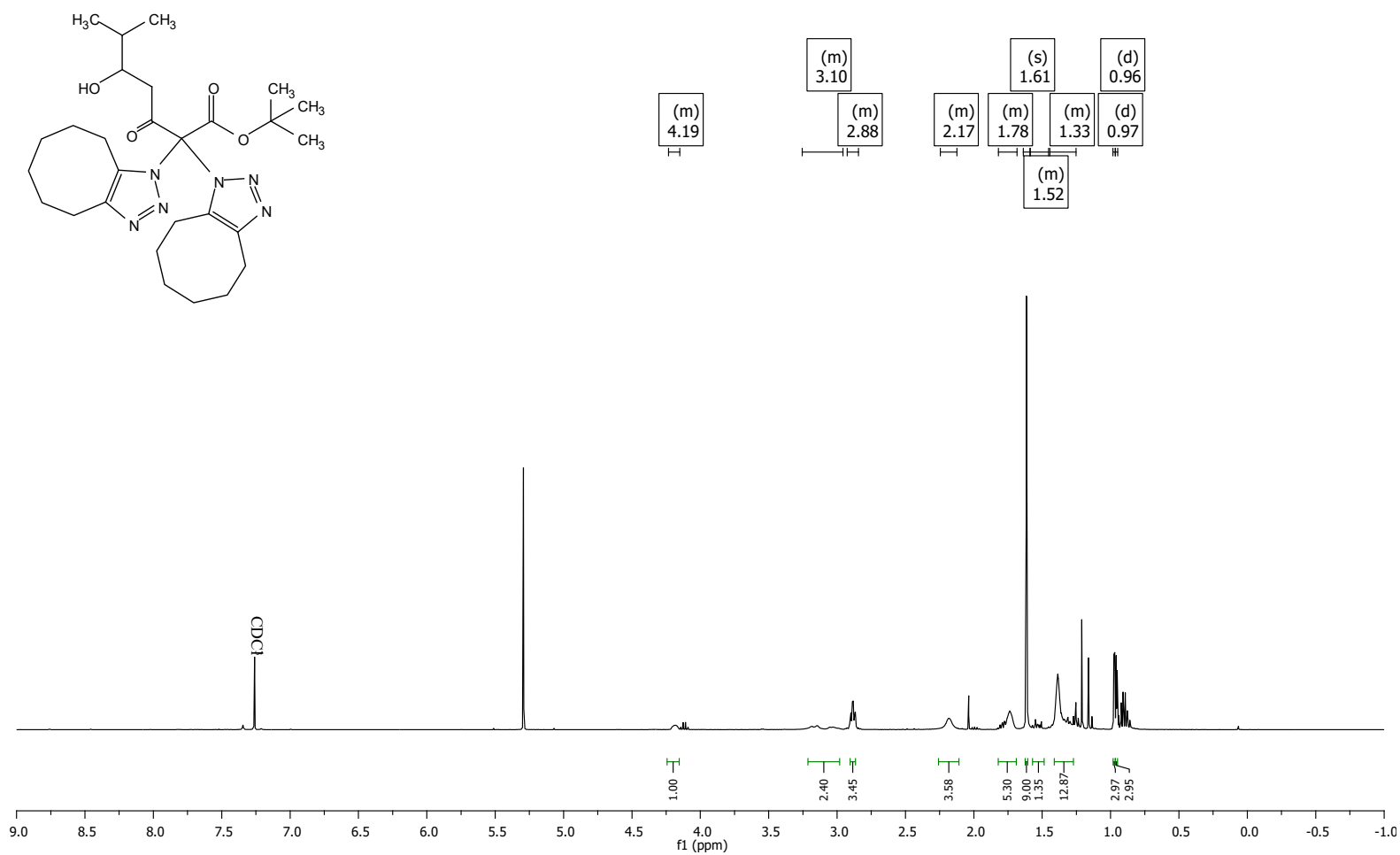


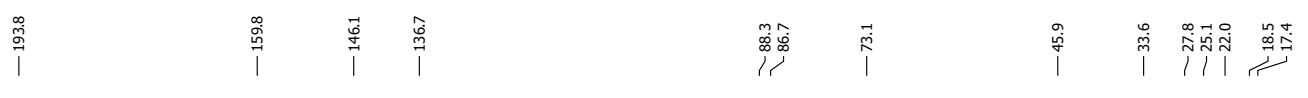

${ }^{13} \mathrm{C}$ NMR $(101 \mathrm{MHz}, \mathrm{CDCl})$
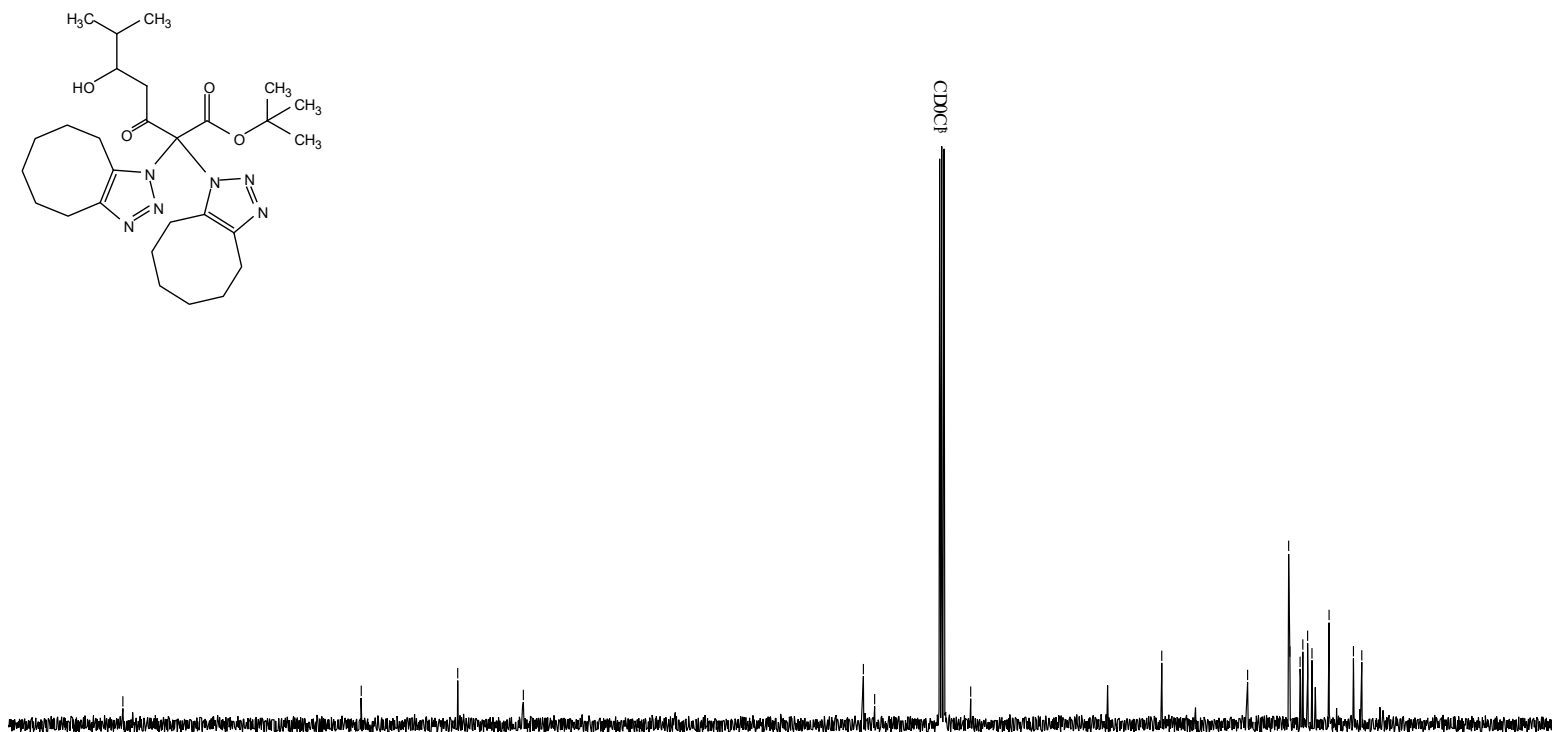

210

$140 \quad 130$
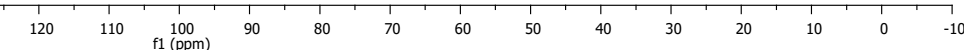

(S)-tert-Butyl 2,2-diazido-4-((tert-butoxycarbonyl)amino)-3-oxopentanoate (2z)

H NMR $(600 \mathrm{MHz}, \mathrm{CDCl})$
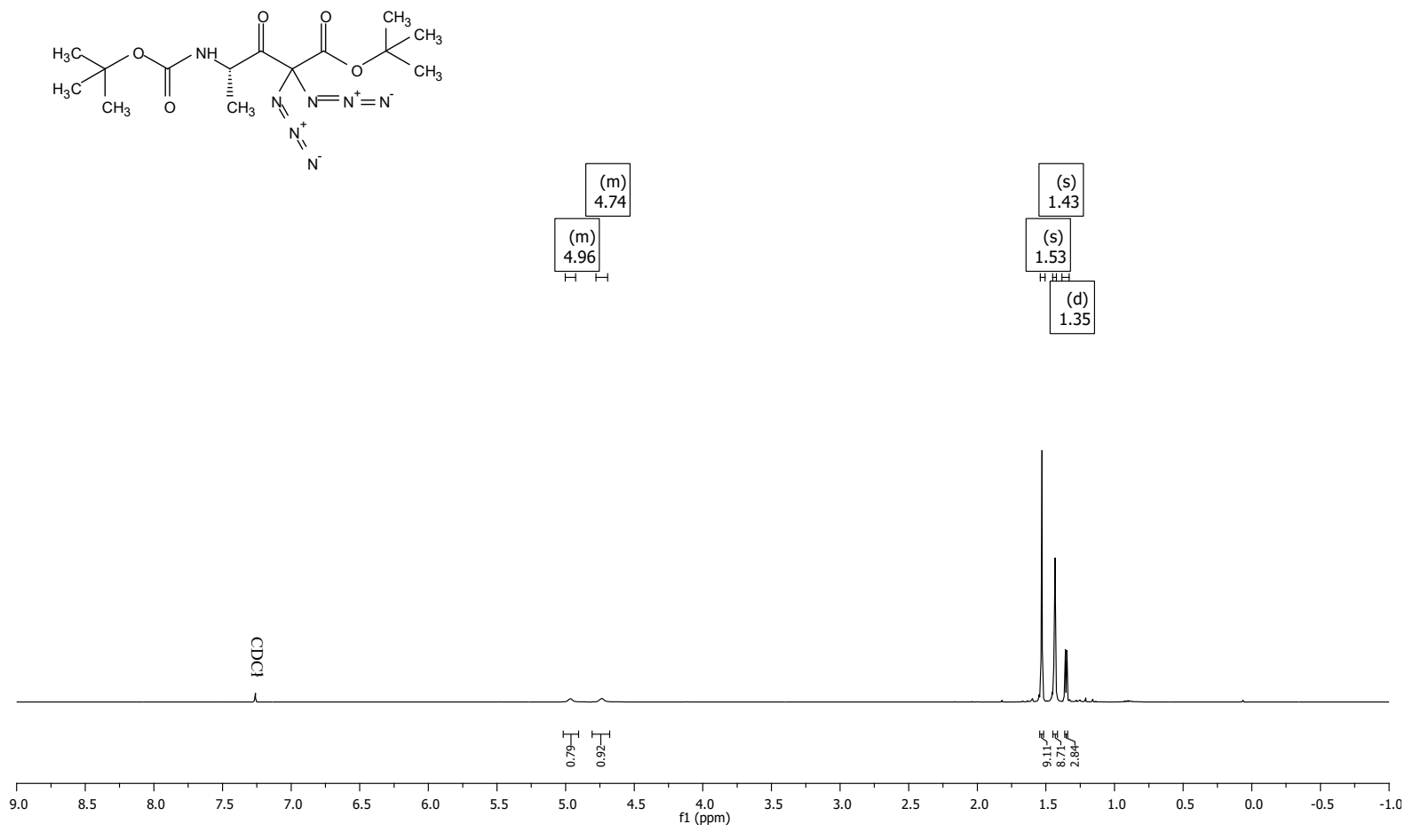
${ }^{13} \mathrm{C} \mathrm{NMR}(151 \mathrm{MHz}$ CDCl)<smiles>C[C@@H](NC(=O)OC(C)(C)C)C(=O)C1(C(=O)OC(C)(C)C)N=NN=C1NN</smiles>

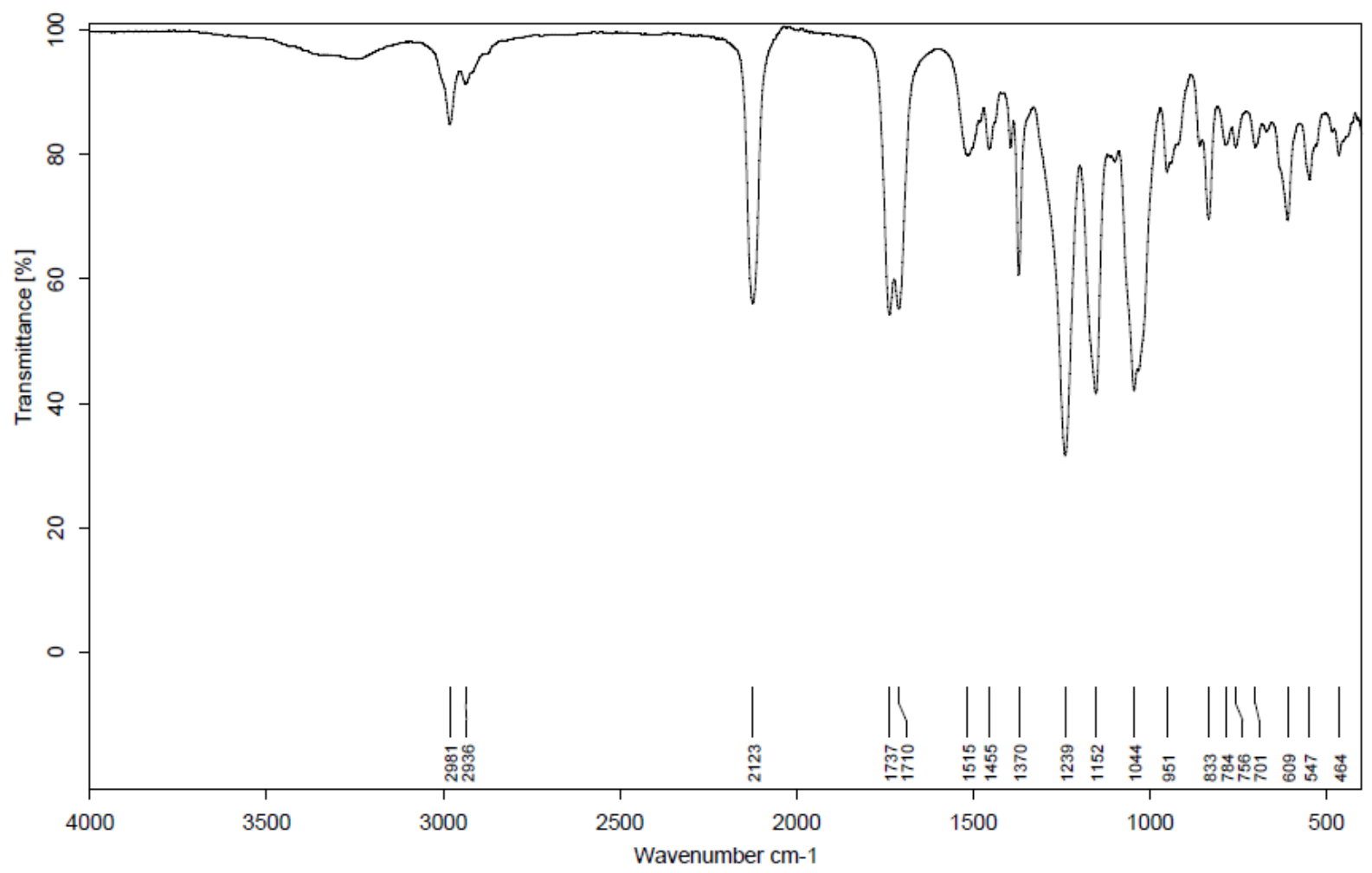


(S)-tert-Butyl 2,2-diazido-4-((tert-butoxycarbonyl)amino)-6-methyl-3-oxoheptanoate (2aa)

'H NMR(400MHz, CDCl)

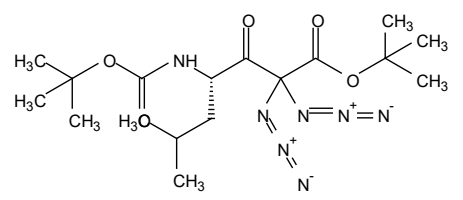

\begin{tabular}{r|}
$(m)$ \\
4.75 \\
\hline
\end{tabular}
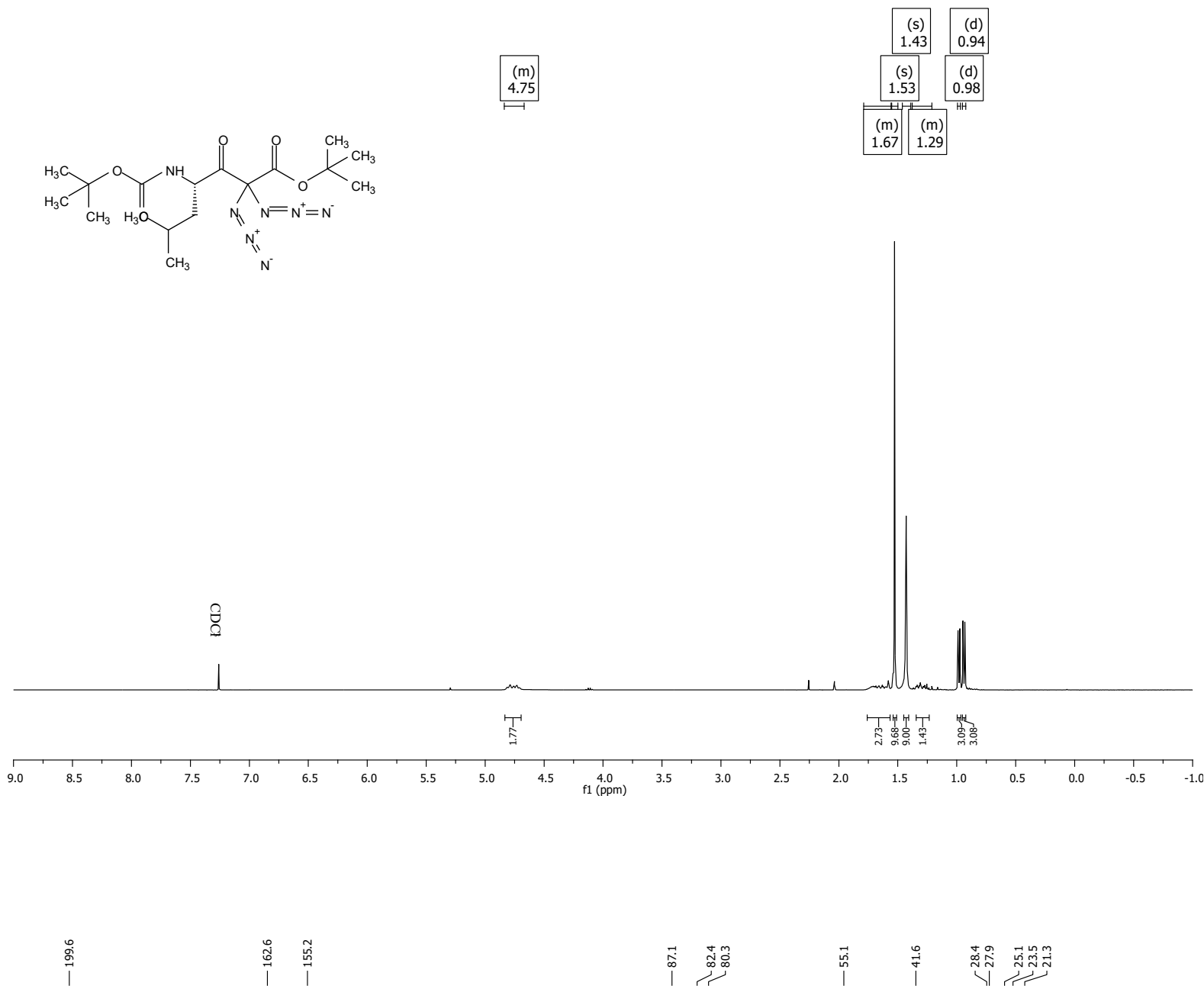

|

${ }^{13} \mathrm{C}$ NMR $(101 \mathrm{MHz}, \mathrm{CDCl})$
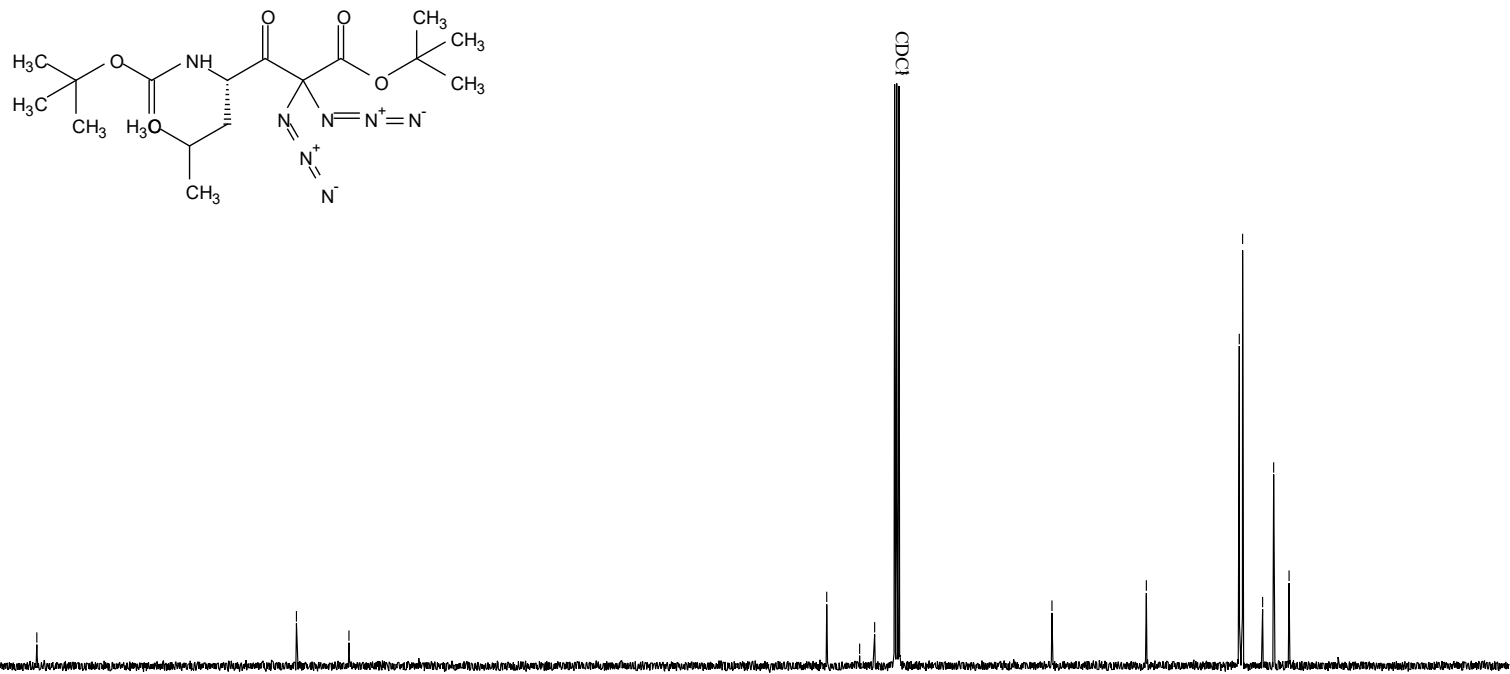


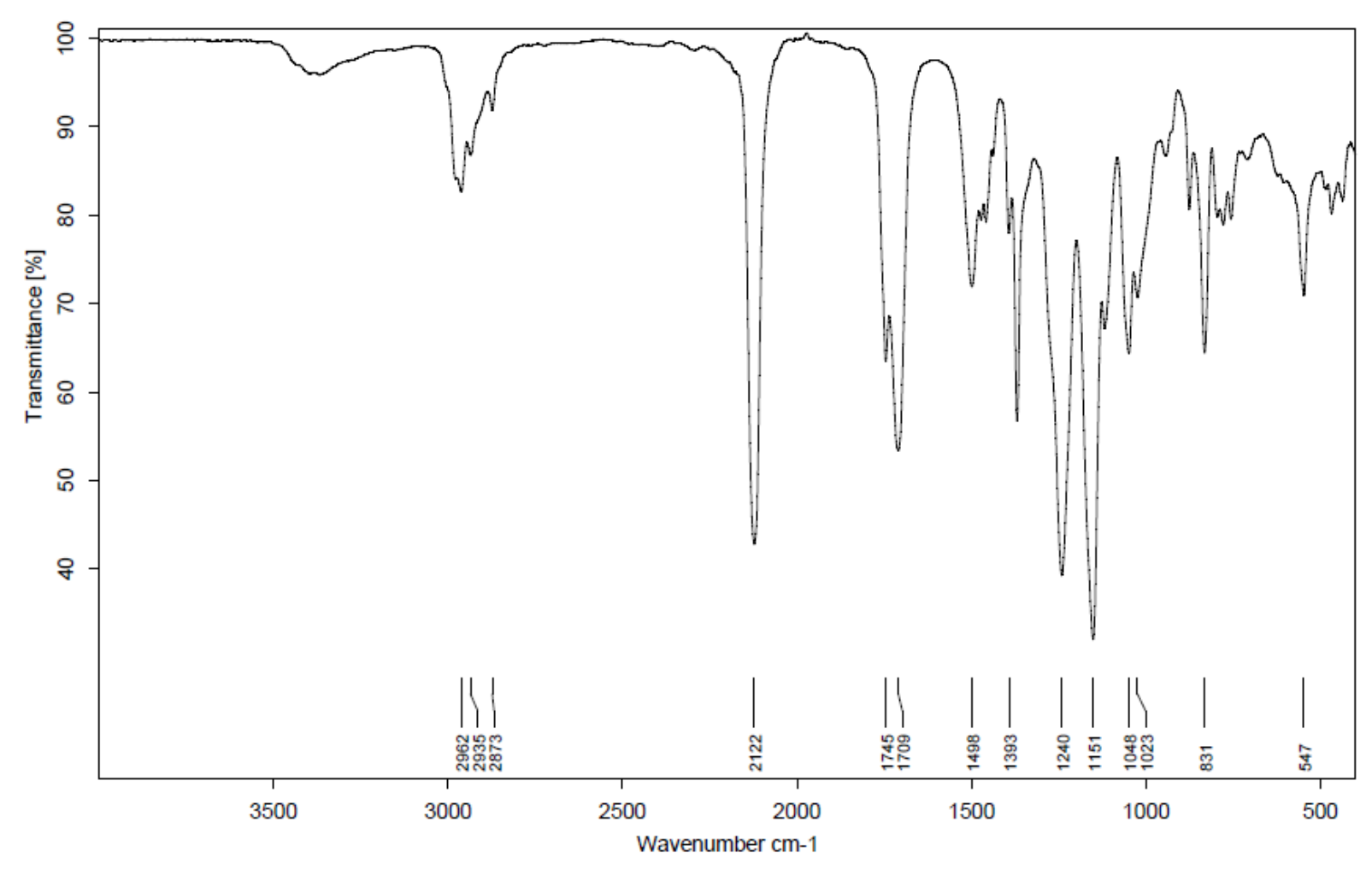

2,2-Diazido- $N, N$-dimethyl-3-oxobutanamide (2ab)

${ }^{1} \mathrm{H} \mathrm{NMR}(400 \mathrm{MHz}, \mathrm{CDCl})$<smiles>CC(=O)C([NH3+])(C(=O)N(C)C)N(C)C</smiles>

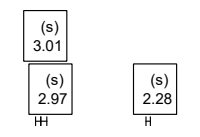

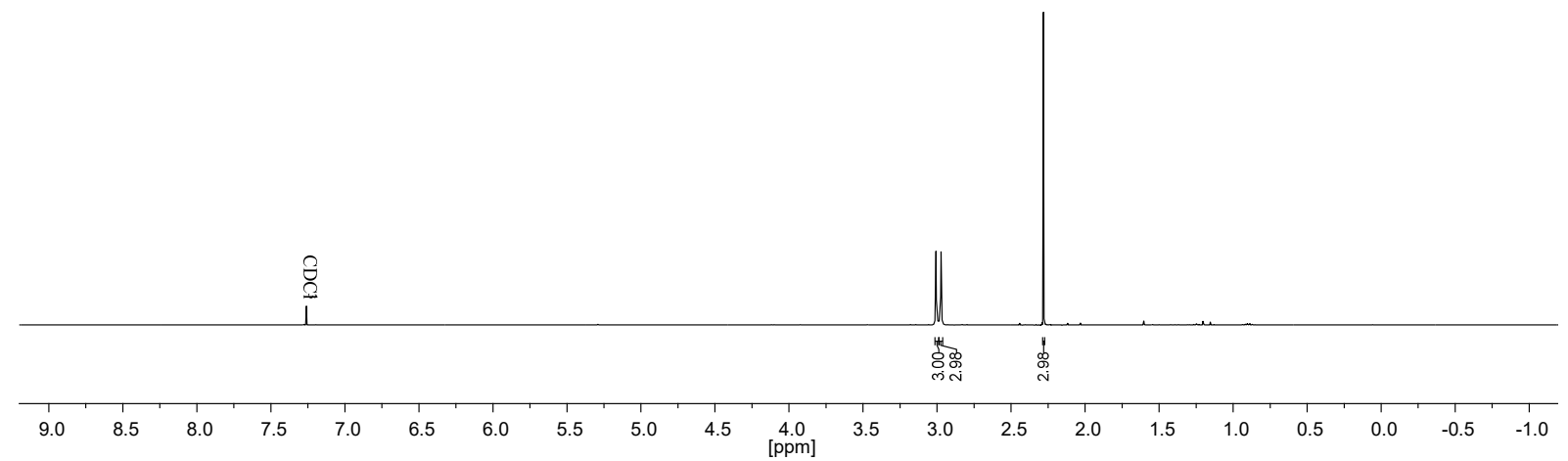



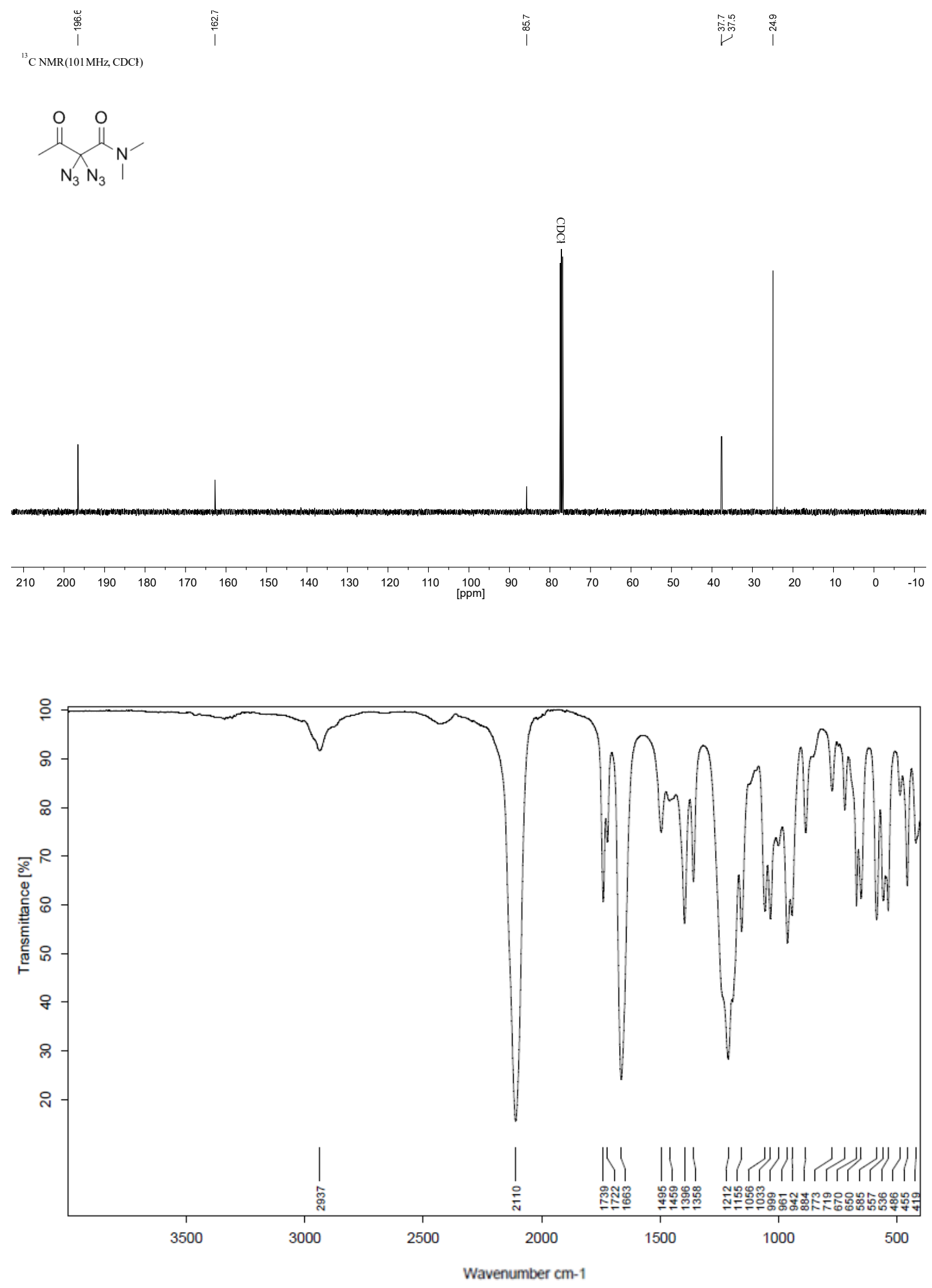


\section{$N, N$-Dimethyl-3-oxo-2,2-bis(4-phenyl-1H-1,2,3-triazol-1-yl)butanamide}

'H NMR (400MHz, CDCl)

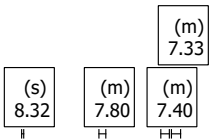

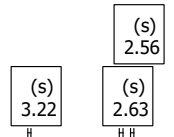

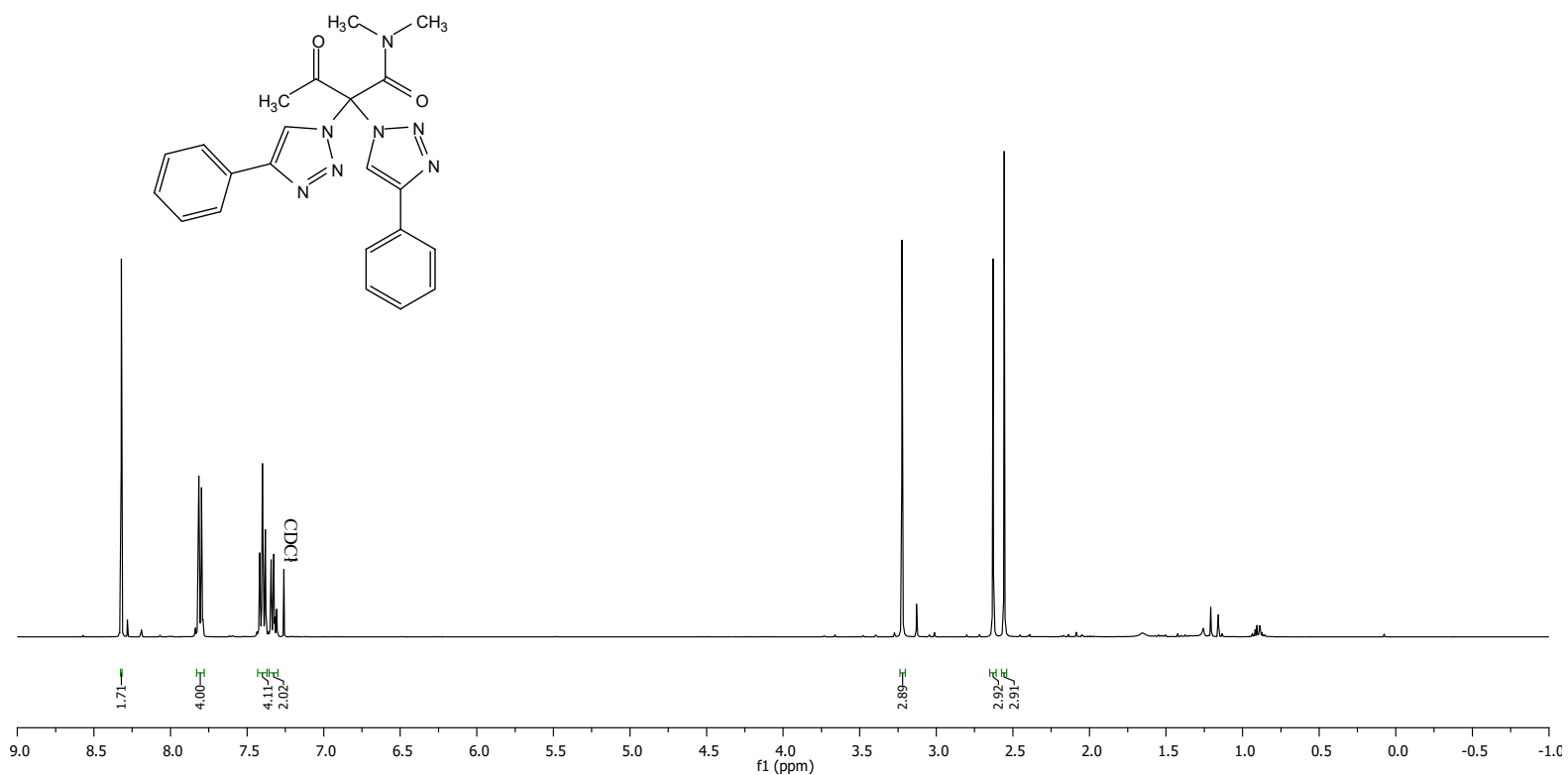

।

${ }^{13} \mathrm{C} \mathrm{NMR}(101 \mathrm{MHz}, \mathrm{CDC} 1)$
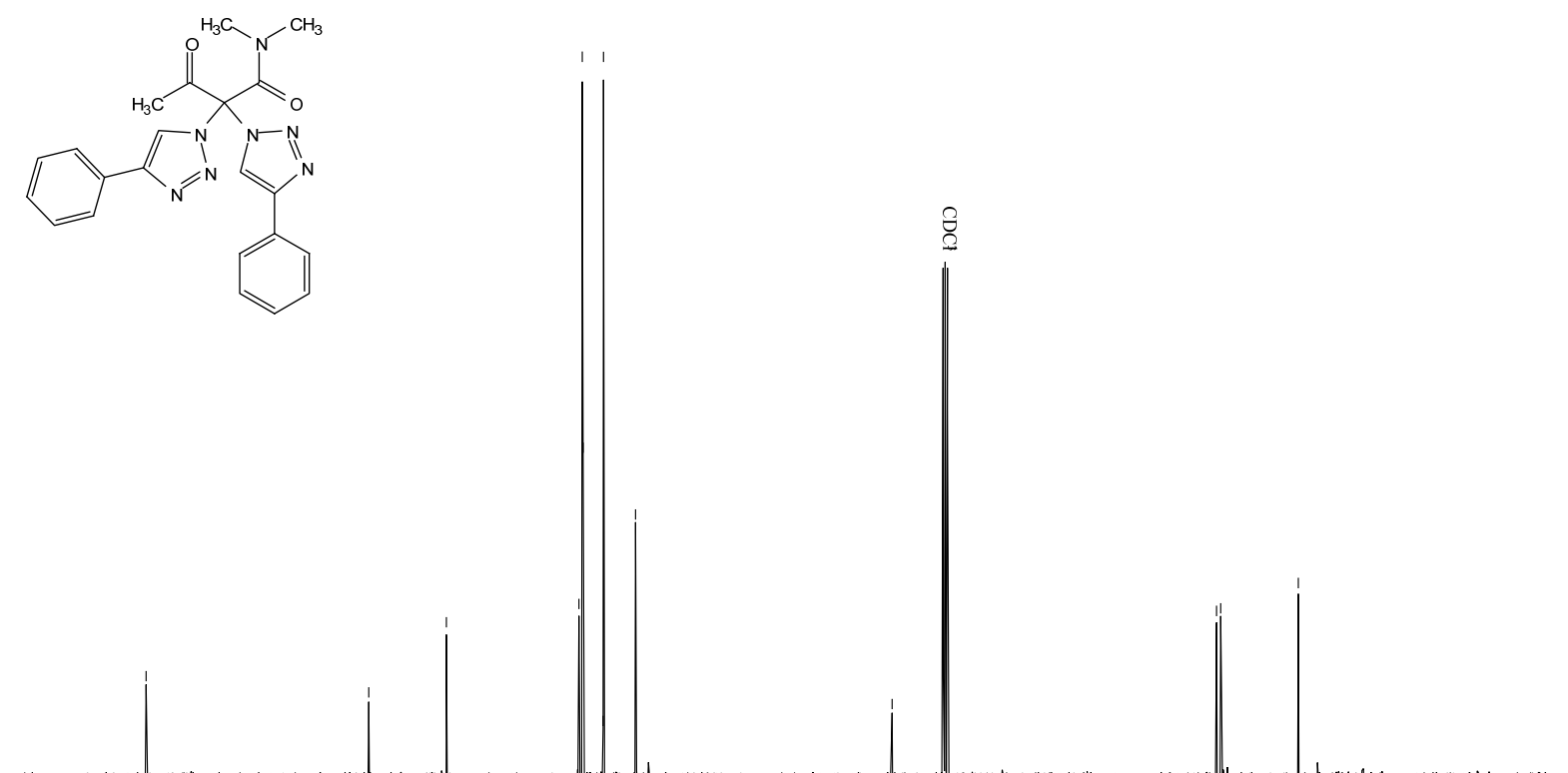

N 
${ }^{1} \mathrm{H}$ NMR(400MHz, CDCl)
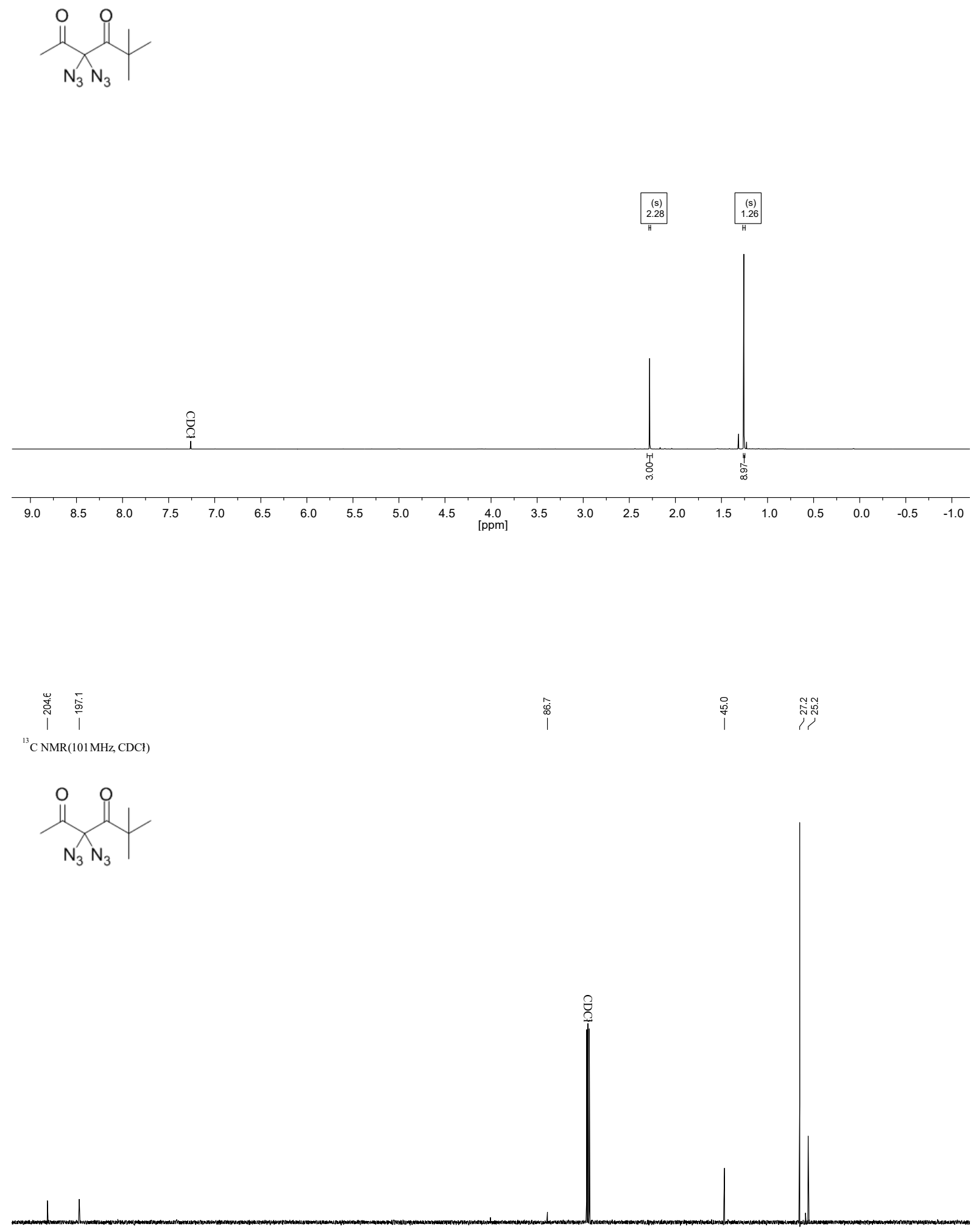

\begin{tabular}{rllllllllllllllllllllll}
\hline 210 & 200 & 190 & 180 & 170 & 160 & 150 & 140 & 130 & 120 & 110 & $\begin{array}{c}100 \\
{[\mathrm{ppm}]}\end{array}$ & 90 & 80 & 70 & 60 & 50 & 40 & 30 & 20 & 10 & 0 & -10
\end{tabular} 


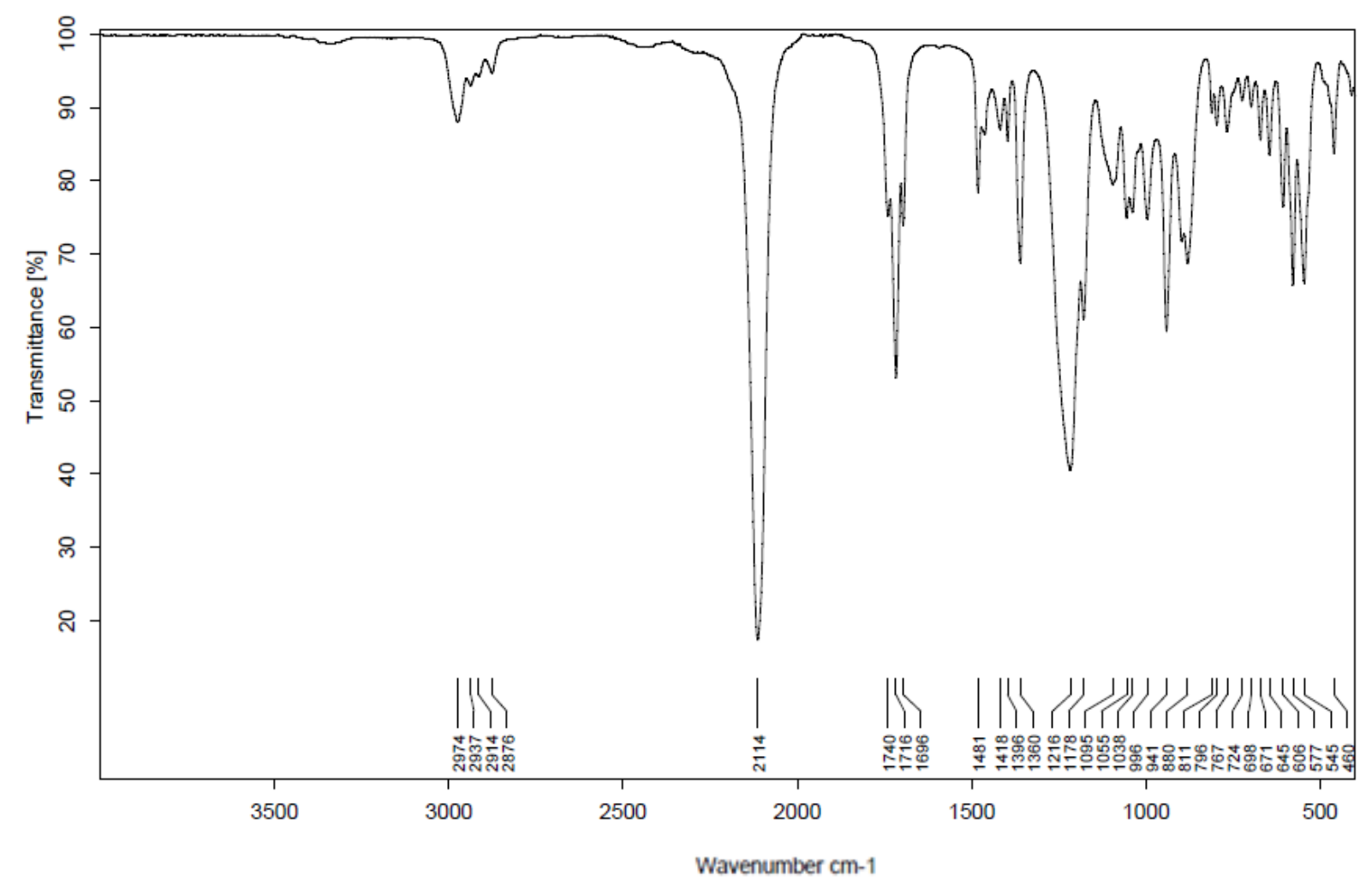

5,5-Dimethyl-3,3-bis(4-phenyl-1H-1,2,3-triazol-1-yl)hexane-2,4-dione

${ }^{1} \mathrm{H}$ NMR(400MHz, CDCl)

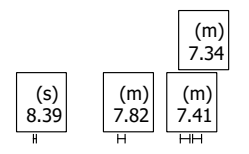
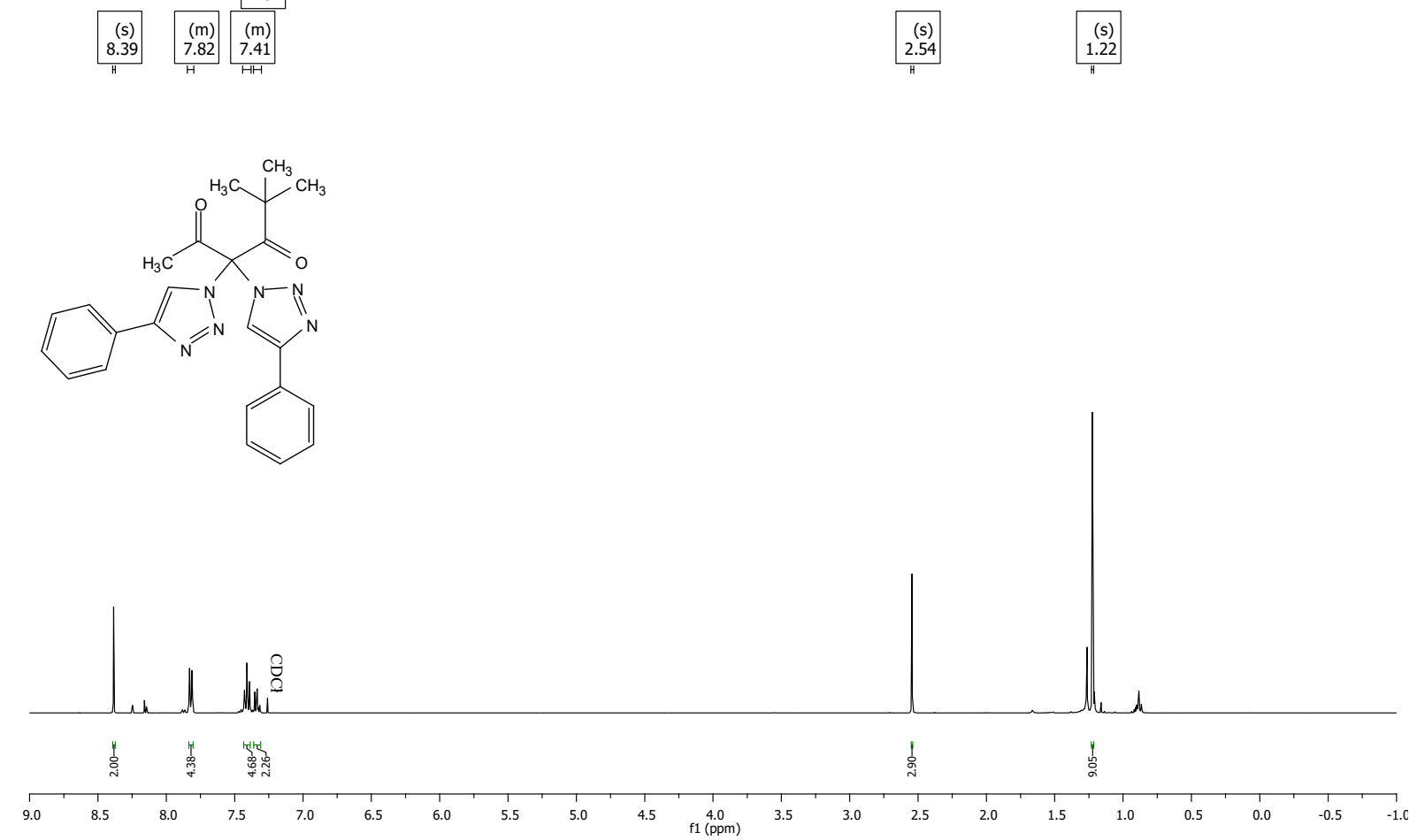

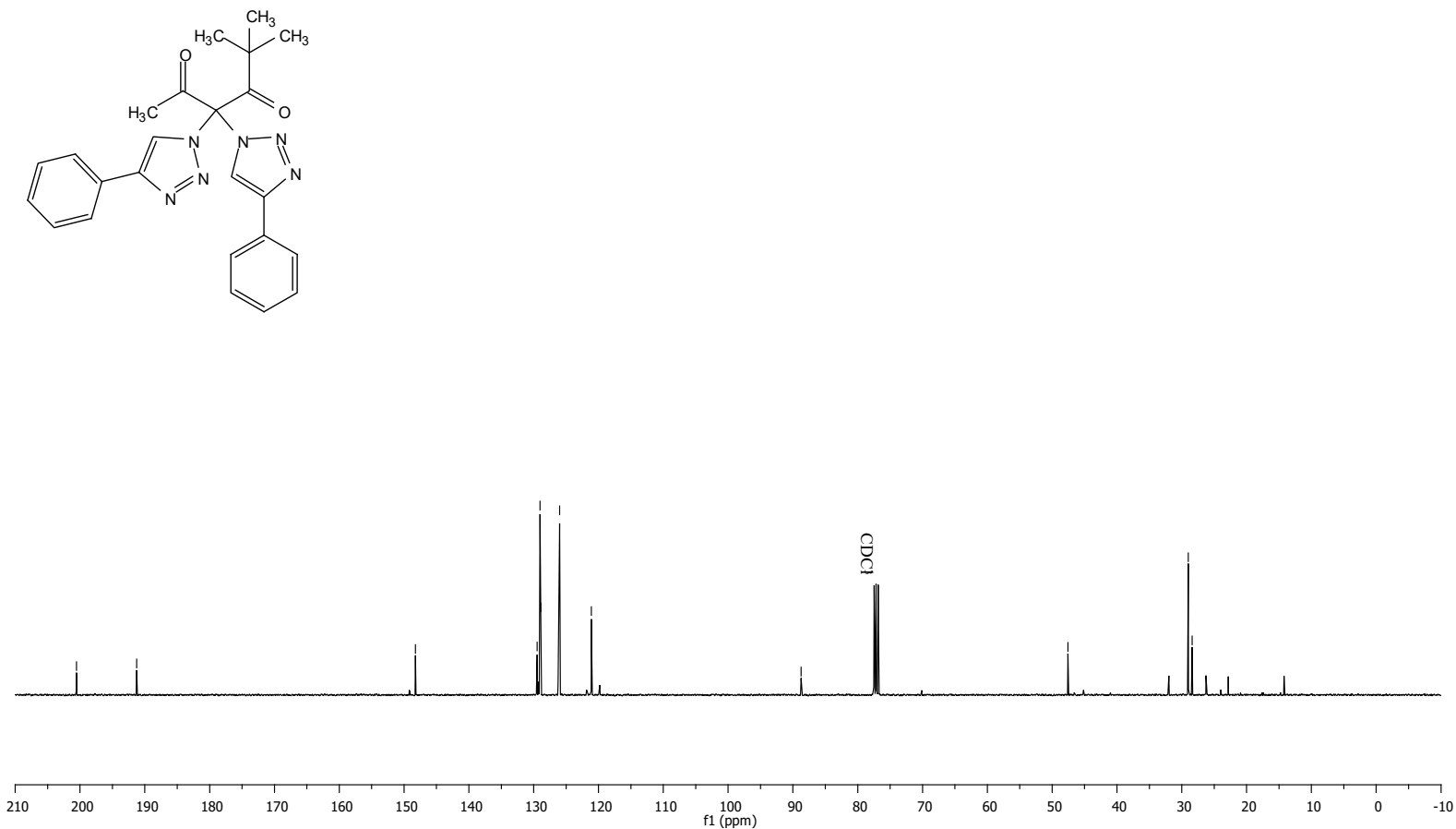

\section{Competition experiment (Scheme 2)}

H NMR(400MHz, CDC1)

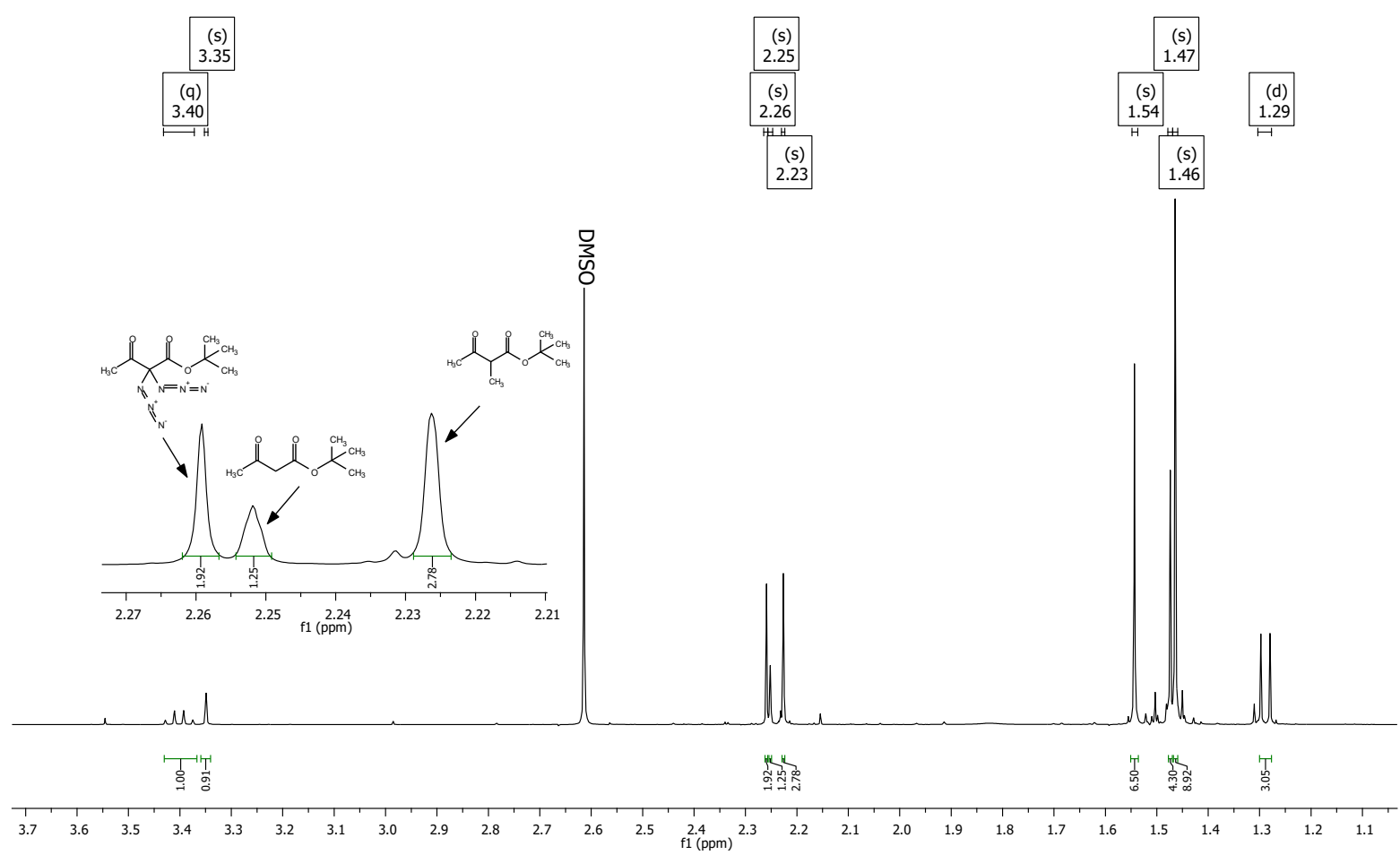




\section{Crystallographic data}

Crystal structure of $\mathbf{2 m}$; ellipsoids are drawn at 50\% probability. Hydrogen atoms are shown as spheres with arbitrary radii.

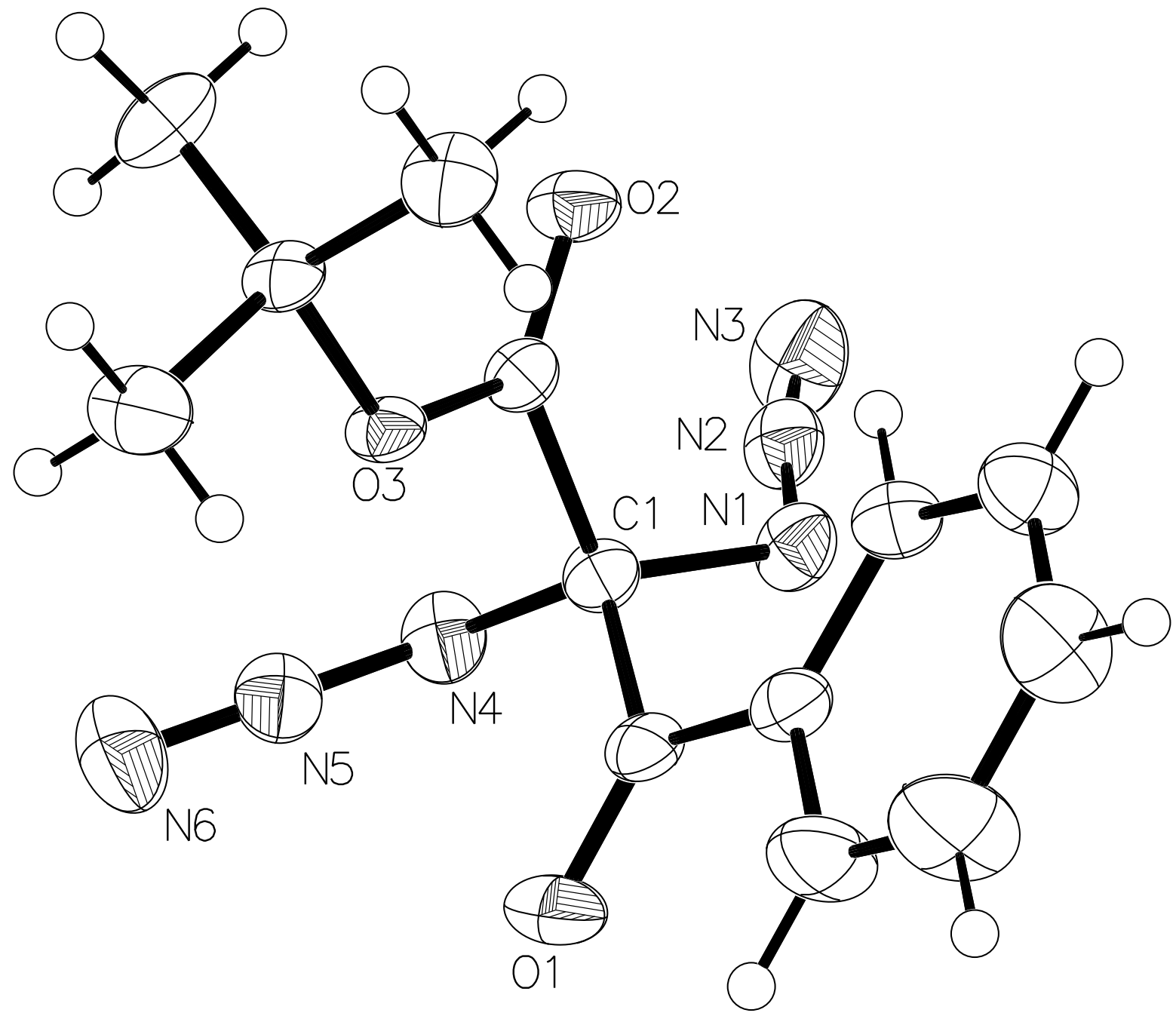

The crystal structure of $\mathbf{2 m}$ was deposited at the Cambridge Crystallographic Data Centre: CCDC 1428570. 
Table 1: Crystal data and structure refinement for $\mathbf{2 m}$.

Empirical formula
Formula weight
Temperature/K
Crystal system
Space group

Space group

$\mathrm{a} / \AA$

$\mathrm{b} / \AA$

$\mathrm{c} / \AA$

$\alpha /^{\circ}$

$\beta /{ }^{\circ}$

$\gamma /{ }^{\circ}$

Volume $/ \AA^{3}$

$\mathrm{Z}$

$\rho_{\text {calc } \mathrm{mg} / \mathrm{mm}^{3}}$

$\mu / \mathrm{mm}^{-1}$

$\mathrm{F}(000)$

Crystal size $/ \mathrm{mm}^{3}$

$2 \Theta$ range for data collection

Index ranges

Reflections collected

Independent reflections

Data/restraints/parameters

Goodness-of-fit on $\mathrm{F}^{2}$

Final $\mathrm{R}$ indexes $[\mathrm{I}>=2 \sigma(\mathrm{I})]$

Final $\mathrm{R}$ indexes [all data]

Largest diff. peak/hole / e $\AA^{-3}$
$\mathrm{C}_{13} \mathrm{H}_{14} \mathrm{~N}_{6} \mathrm{O}_{3}$

302.30

150

triclinic

P-1

$8.3623(5)$

9.5799(5)

$10.5979(6)$

103.936(5)

91.136(5)

111.334(5)

$762.10(7)$

2

1.317

0.098

316.0

$0.11 \times 0.09 \times 0.05$

3.98 to $58.92^{\circ}$

$-10 \leq \mathrm{h} \leq 10,-13 \leq \mathrm{k} \leq 12,-14 \leq 1 \leq 14$

6417

$3519[\mathrm{R}($ int $)=0.0189]$

$3519 / 0 / 202$

1.032

$\mathrm{R}_{1}=0.0412, \mathrm{wR}_{2}=0.0929$

$\mathrm{R}_{1}=0.0562, \mathrm{w}_{2}=0.1015$

$0.29 /-0.19$ 
Table 2: Fractional Atomic Coordinates $\left(\times 10^{4}\right)$ and Equivalent Isotropic Displacement Parameters $\left(\AA^{2} \times 10^{3}\right)$ for $\mathbf{2 m}$. $U_{\text {eq }}$ is defined as $1 / 3$ of the trace of the orthogonalised UIJ tensor.

\begin{tabular}{ccccc}
\hline Atom & $\boldsymbol{x}$ & $\boldsymbol{y}$ & $\boldsymbol{z}$ & $\mathbf{U}(\mathbf{e q})$ \\
$\mathrm{O} 3$ & $1049.9(11)$ & $8443.8(10)$ & $7032.7(8)$ & $24.5(2)$ \\
O2 & $92.5(12)$ & $6820.0(11)$ & $4981.9(9)$ & $31.4(2)$ \\
O1 & $-2418.9(14)$ & $8836.6(12)$ & $8426.4(10)$ & $39.6(3)$ \\
$\mathrm{N} 1$ & $-3317.5(14)$ & $6541.5(13)$ & $5468.6(11)$ & $30.5(3)$ \\
$\mathrm{N} 4$ & $-1696.3(15)$ & $9265.8(13)$ & $6036.1(12)$ & $32.3(3)$ \\
$\mathrm{C} 9$ & $-103.0(16)$ & $7583.4(14)$ & $5998.1(12)$ & $23.2(3)$ \\
$\mathrm{N} 5$ & $-636.0(16)$ & $10445.0(14)$ & $6831.0(13)$ & $34.3(3)$ \\
N2 & $-3423.2(14)$ & $6574.4(13)$ & $4301.3(12)$ & $31.2(3)$ \\
C2 & $-2204.8(16)$ & $7731.5(15)$ & $7725.1(13)$ & $26.8(3)$ \\
C1 & $-1824.9(16)$ & $7779.8(14)$ & $6297.9(13)$ & $24.5(3)$ \\
C3 & $-2290.6(16)$ & $6349.5(15)$ & $8157.1(13)$ & $27.7(3)$ \\
C10 & $2855.2(16)$ & $8478.2(15)$ & $7129.7(13)$ & $25.5(3)$ \\
C8 & $-2078.6(18)$ & $5065.6(16)$ & $7350.6(14)$ & $31.6(3)$ \\
N3 & $-3725.3(18)$ & $6456.5(17)$ & $3235.8(14)$ & $48.0(4)$ \\
C13 & $2741.1(19)$ & $6869.5(17)$ & $7142.0(16)$ & $37.4(3)$ \\
C11 & $3764.0(18)$ & $9026.8(19)$ & $6017.2(15)$ & $39.6(4)$ \\
C7 & $-2135(2)$ & $3829.3(18)$ & $7830.9(15)$ & $40.1(4)$ \\
N6 & $277.0(19)$ & $11596.4(15)$ & $7467.3(15)$ & $50.0(4)$ \\
C4 & $-2577(2)$ & $6363(2)$ & $9449.9(15)$ & $46.1(4)$ \\
C6 & $-2417(3)$ & $3865(2)$ & $9111.0(17)$ & $53.0(5)$ \\
C12 & $3647(2)$ & $9650(2)$ & $8429.2(16)$ & $45.1(4)$ \\
C5 & $-2642(3)$ & $5123(2)$ & $9916.0(17)$ & $63.5(6)$ \\
\hline & & & &
\end{tabular}


Table 3: Anisotropic Displacement Parameters $\left(\AA^{2} \times 10^{3}\right)$ for $\mathbf{2 m}$. The Anisotropic displacement factor exponent takes the form: $2 \pi^{2}\left[h^{2} a^{* 2} U 11+\ldots+2 h k a \times b \times U_{12}\right]$.

\begin{tabular}{ccccccc}
\hline Atom & $\mathbf{U}_{\mathbf{1 1}}$ & $\mathbf{U}_{\mathbf{2 2}}$ & $\mathbf{U}_{\mathbf{3 3}}$ & $\mathbf{U}_{\mathbf{2 3}}$ & $\mathbf{U}_{\mathbf{1 3}}$ & $\mathbf{U}_{\mathbf{1 2}}$ \\
$\mathrm{O} 3$ & $17.8(4)$ & $31.9(5)$ & $23.5(5)$ & $3.0(4)$ & $1.0(3)$ & $12.1(4)$ \\
$\mathrm{O} 2$ & $28.7(5)$ & $41.4(5)$ & $23.5(5)$ & $2.9(4)$ & $3.3(4)$ & $16.1(4)$ \\
$\mathrm{O} 1$ & $42.5(6)$ & $40.9(6)$ & $40.7(6)$ & $5.3(5)$ & $14.3(5)$ & $24.9(5)$ \\
$\mathrm{N} 1$ & $22.1(6)$ & $36.6(6)$ & $29.2(6)$ & $8.3(5)$ & $-1.4(5)$ & $7.7(5)$ \\
$\mathrm{N} 4$ & $26.4(6)$ & $32.3(6)$ & $40.0(7)$ & $10.3(5)$ & $-3.1(5)$ & $13.0(5)$ \\
$\mathrm{C} 9$ & $19.8(6)$ & $26.7(6)$ & $24.6(6)$ & $8.7(5)$ & $2.2(5)$ & $9.5(5)$ \\
$\mathrm{N} 5$ & $30.9(6)$ & $31.0(6)$ & $45.8(7)$ & $12.0(6)$ & $2.3(6)$ & $16.3(6)$ \\
N2 & $22.7(6)$ & $33.3(6)$ & $34.4(7)$ & $7.5(5)$ & $-2.5(5)$ & $8.3(5)$ \\
C2 & $16.9(6)$ & $32.5(7)$ & $29.9(7)$ & $4.1(6)$ & $3.6(5)$ & $10.6(5)$ \\
C1 & $19.4(6)$ & $27.0(6)$ & $28.4(7)$ & $6.9(5)$ & $1.2(5)$ & $10.6(5)$ \\
C3 & $22.3(6)$ & $34.3(7)$ & $25.5(7)$ & $6.7(6)$ & $4.2(5)$ & $10.4(6)$ \\
C10 & $17.4(6)$ & $33.7(7)$ & $27.9(7)$ & $6.8(6)$ & $0.6(5)$ & $13.6(5)$ \\
C8 & $33.1(7)$ & $37.0(7)$ & $26.8(7)$ & $8.8(6)$ & $6.8(6)$ & $15.4(6)$ \\
N3 & $39.0(8)$ & $57.6(9)$ & $36.6(8)$ & $13.7(7)$ & $-7.6(6)$ & $5.9(7)$ \\
C13 & $30.4(7)$ & $40.7(8)$ & $48.0(9)$ & $15.4(7)$ & $0.9(6)$ & $19.2(7)$ \\
C11 & $24.3(7)$ & $56.7(9)$ & $45.0(9)$ & $24.7(8)$ & $9.8(6)$ & $16.1(7)$ \\
C7 & $49.9(9)$ & $37.5(8)$ & $37.2(8)$ & $10.5(7)$ & $5.8(7)$ & $21.2(7)$ \\
N6 & $50.5(9)$ & $31.4(7)$ & $63.5(10)$ & $7.9(7)$ & $-5.0(7)$ & $13.7(7)$ \\
C4 & $65.6(11)$ & $46.2(9)$ & $29.3(8)$ & $8.2(7)$ & $14.5(8)$ & $25.1(8)$ \\
C6 & $76.2(13)$ & $47.3(10)$ & $41.7(10)$ & $22.1(8)$ & $5.5(9)$ & $23.9(9)$ \\
C12 & $28.7(8)$ & $57(1)$ & $40.6(9)$ & $-7.1(8)$ & $-8.1(7)$ & $19.5(7)$ \\
C5 & $105.8(17)$ & $62.5(11)$ & $30.9(9)$ & $20.5(9)$ & $21.3(10)$ & $36.0(12)$ \\
\hline
\end{tabular}

Table 4: Bond Lengths for $\mathbf{2 m}$.

\begin{tabular}{|c|c|c|c|c|c|}
\hline Atom & Atom & Length/Å & Atom & Atom & Length/Å \\
\hline $\mathrm{O} 3$ & C9 & $1.3250(15)$ & $\mathrm{C} 2$ & $\mathrm{C} 1$ & $1.5598(18)$ \\
\hline $\mathrm{O} 3$ & $\mathrm{C} 10$ & $1.4989(14)$ & $\mathrm{C} 2$ & $\mathrm{C} 3$ & $1.4804(19)$ \\
\hline $\mathrm{O} 2$ & C9 & $1.1984(15)$ & $\mathrm{C} 3$ & $\mathrm{C} 8$ & $1.3904(19)$ \\
\hline $\mathrm{O} 1$ & $\mathrm{C} 2$ & $1.2122(16)$ & $\mathrm{C} 3$ & $\mathrm{C} 4$ & $1.393(2)$ \\
\hline N1 & $\mathrm{N} 2$ & $1.2473(16)$ & $\mathrm{C} 10$ & $\mathrm{C} 13$ & $1.5124(19)$ \\
\hline N1 & $\mathrm{C} 1$ & $1.4541(16)$ & $\mathrm{C} 10$ & $\mathrm{C} 11$ & $1.5123(19)$ \\
\hline N4 & N5 & $1.2471(17)$ & $\mathrm{C} 10$ & $\mathrm{C} 12$ & $1.5079(19)$ \\
\hline N4 & $\mathrm{C} 1$ & $1.4816(17)$ & $\mathrm{C} 8$ & $\mathrm{C} 7$ & $1.384(2)$ \\
\hline $\mathrm{C} 9$ & $\mathrm{C} 1$ & $1.5480(17)$ & $\mathrm{C} 7$ & C6 & $1.376(2)$ \\
\hline N5 & N6 & $1.1238(17)$ & $\mathrm{C} 4$ & C5 & $1.377(2)$ \\
\hline $\mathrm{N} 2$ & N3 & $1.1217(17)$ & C6 & $\mathrm{C} 5$ & $1.376(3)$ \\
\hline
\end{tabular}


Table 5: Bond Angles for $\mathbf{2 m}$.

\begin{tabular}{ccccccccc}
\hline Atom & Atom Atom & \multicolumn{1}{c}{ Angle $^{\circ}$} & \multicolumn{2}{c}{ Atom } & Atom Atom & \multicolumn{1}{c}{ Angle $^{\circ}$} \\
C9 & O3 & C10 & $122.50(10)$ & C9 & C1 & C2 & $112.06(10)$ \\
N2 & N1 & C1 & $114.68(11)$ & C8 & C3 & C2 & $123.56(12)$ \\
N5 & N4 & C1 & $114.16(11)$ & C8 & C3 & C4 & $118.97(13)$ \\
O3 & C9 & C1 & $107.20(10)$ & C4 & C3 & C2 & $117.47(13)$ \\
O2 & C9 & O3 & $128.79(12)$ & O3 & C10 & C13 & $107.77(10)$ \\
O2 & C9 & C1 & $123.97(11)$ & O3 & C10 & C11 & $110.01(10)$ \\
N6 & N5 & N4 & $172.57(14)$ & O3 & C10 & C12 & $102.05(10)$ \\
N3 & N2 & N1 & $170.97(14)$ & C11 & C10 & C13 & $113.04(12)$ \\
O1 & C2 & C1 & $117.87(12)$ & C12 & C10 & C13 & $112.03(12)$ \\
O1 & C2 & C3 & $123.26(13)$ & C12 & C10 & C11 & $111.30(13)$ \\
C3 & C2 & C1 & $118.87(11)$ & C7 & C8 & C3 & $120.38(13)$ \\
N1 & C1 & N4 & $108.10(10)$ & C6 & C7 & C8 & $119.83(15)$ \\
N1 & C1 & C9 & $112.80(11)$ & C5 & C4 & C3 & $120.20(15)$ \\
N1 & C1 & C2 & $104.86(10)$ & C5 & C6 & C7 & $120.35(16)$ \\
N4 & C1 & C9 & $106.68(10)$ & C6 & C5 & C4 & $120.27(16)$ \\
N4 & C1 & C2 & $112.39(11)$ & & & & \\
\hline
\end{tabular}

Table 6: Hydrogen Atom Coordinates $\left(\AA \AA \times 10^{4}\right)$ and Isotropic Displacement Parameters $\left(\AA^{2} \times 10^{3}\right)$ for $\mathbf{2 m}$.

\begin{tabular}{ccccc}
\hline Atom & $\boldsymbol{x}$ & $\boldsymbol{y}$ & $\boldsymbol{z}$ & $\mathbf{U}(\mathbf{e q )}$ \\
H8 & -1894 & 5037 & 6465 & 38 \\
H13A & 2062 & 6542 & 7836 & 56 \\
H13B & 3907 & 6887 & 7304 & 56 \\
H13C & 2180 & 6137 & 6294 & 56 \\
H11A & 3256 & 8224 & 5192 & 59 \\
H11B & 4996 & 9225 & 6172 & 59 \\
H11C & 3629 & 9987 & 5967 & 59 \\
H7 & -1981 & 2958 & 7278 & 48 \\
H4 & -2729 & 7231 & 10011 & 55 \\
H6 & -2455 & 3016 & 9441 & 64 \\
H12A & 3670 & 10668 & 8388 & 68 \\
H12B & 4829 & 9724 & 8627 & 68 \\
H12C & 2956 & 9320 & 9117 & 68 \\
H5 & -2843 & 5136 & 10797 & 76 \\
\hline
\end{tabular}




\section{Experimental:}

The crystal was kept at $150 \mathrm{~K}$ during data collection. Using Olex2 [1], the structure was solved with the ShelXS ${ }^{[2]}$ structure solution program using Direct Methods and refined with the ShelXL ${ }^{[3]}$ refinement package using Least Squares minimisation.

[1] O. V. Dolomanov, L. J. Bourhis, R. J. Gildea, J. A. K. Howard and H. Puschmann, OLEX2: a complete structure solution, refinement and analysis program. J. Appl. Cryst. 2009, 42, 339.

[2] SHELXS, G.M. Sheldrick, Acta Cryst. 2008, A64, 112.

[3] SHELXL, G.M. Sheldrick, Acta Cryst. 2008, A64, 112.

Crystal structure determination of $\mathbf{2 m}$ :

Crystal Data for $\mathrm{C}_{13} \mathrm{H}_{14} \mathrm{~N}_{6} \mathrm{O}_{3}(M=302.30)$ : triclinic, space group P-1 (no. 2), $a=8.3623(5) \AA, b=$ 9.5799(5) $\AA, c=\quad 10.5979(6) \AA, \alpha=\quad 103.936(5)^{\circ}, \beta=\quad 91.136(5)^{\circ}, \gamma=\quad 111.334(5)^{\circ}, V=$ $762.10(7) \AA^{3}, Z=2, T=150 \mathrm{~K}, \mu(\mathrm{Mo} \mathrm{K \alpha})=0.098 \mathrm{~mm}^{-1}$, Dcalc $=1.317 \mathrm{~g} / \mathrm{mm}^{3}, 6417$ reflections measured $(3.98 \leq 2 \Theta \leq 58.92), 3519$ unique $\left(R_{\text {int }}=0.0189\right)$ which were used in all calculations. The final $R_{1}$ was $0.0412(>2 \operatorname{sigma}(\mathrm{I}))$ and $w R_{2}$ was 0.1015 . 
TGA-DSC measurements

di-tert-butyl 2,2-diazidomalonate (2c)
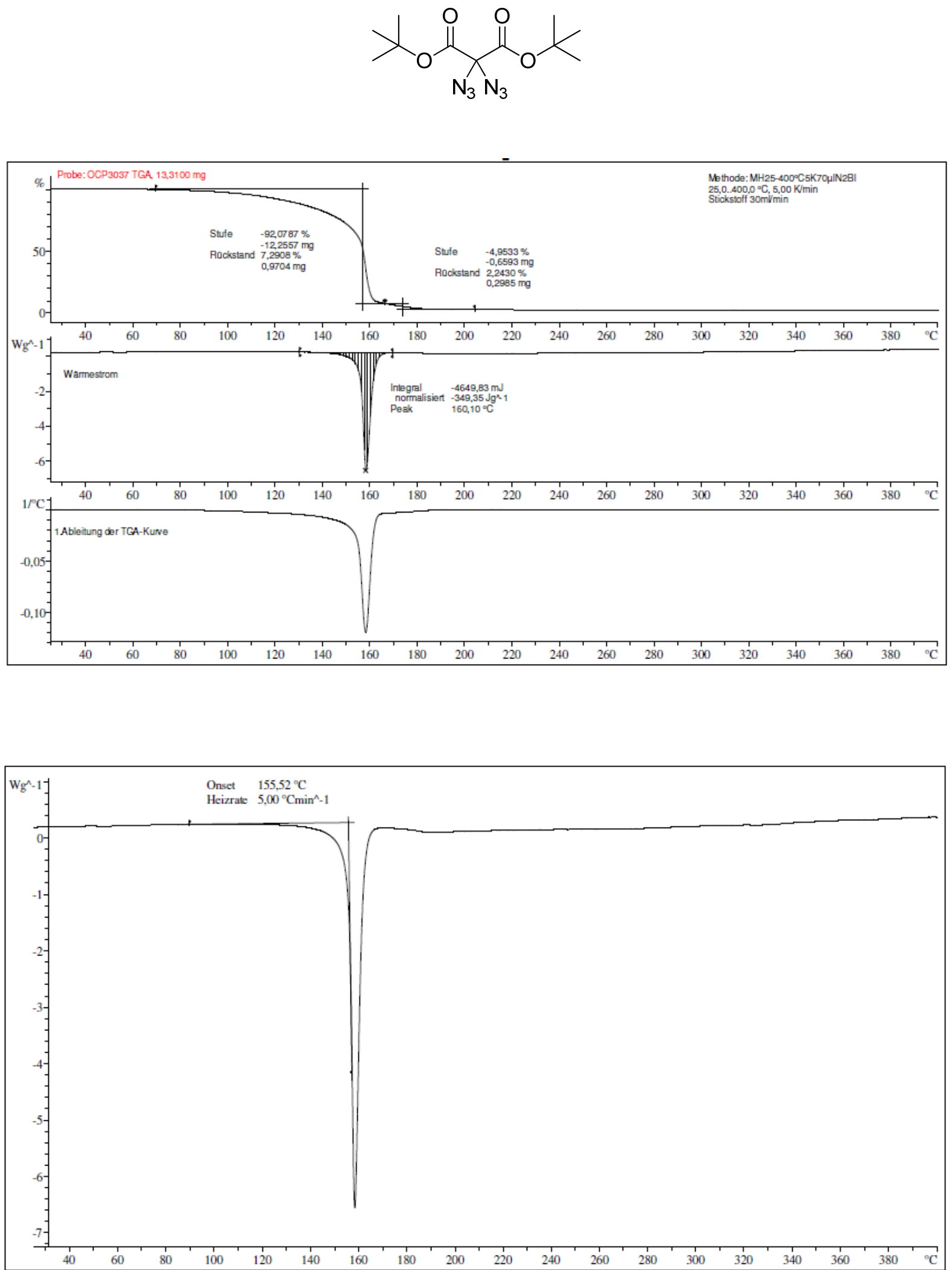
tert-butyl 2,2diazido-3-oxobutanoate (2g)<smiles>CCCCOC(=O)C(N)(N)C(C)=O</smiles>
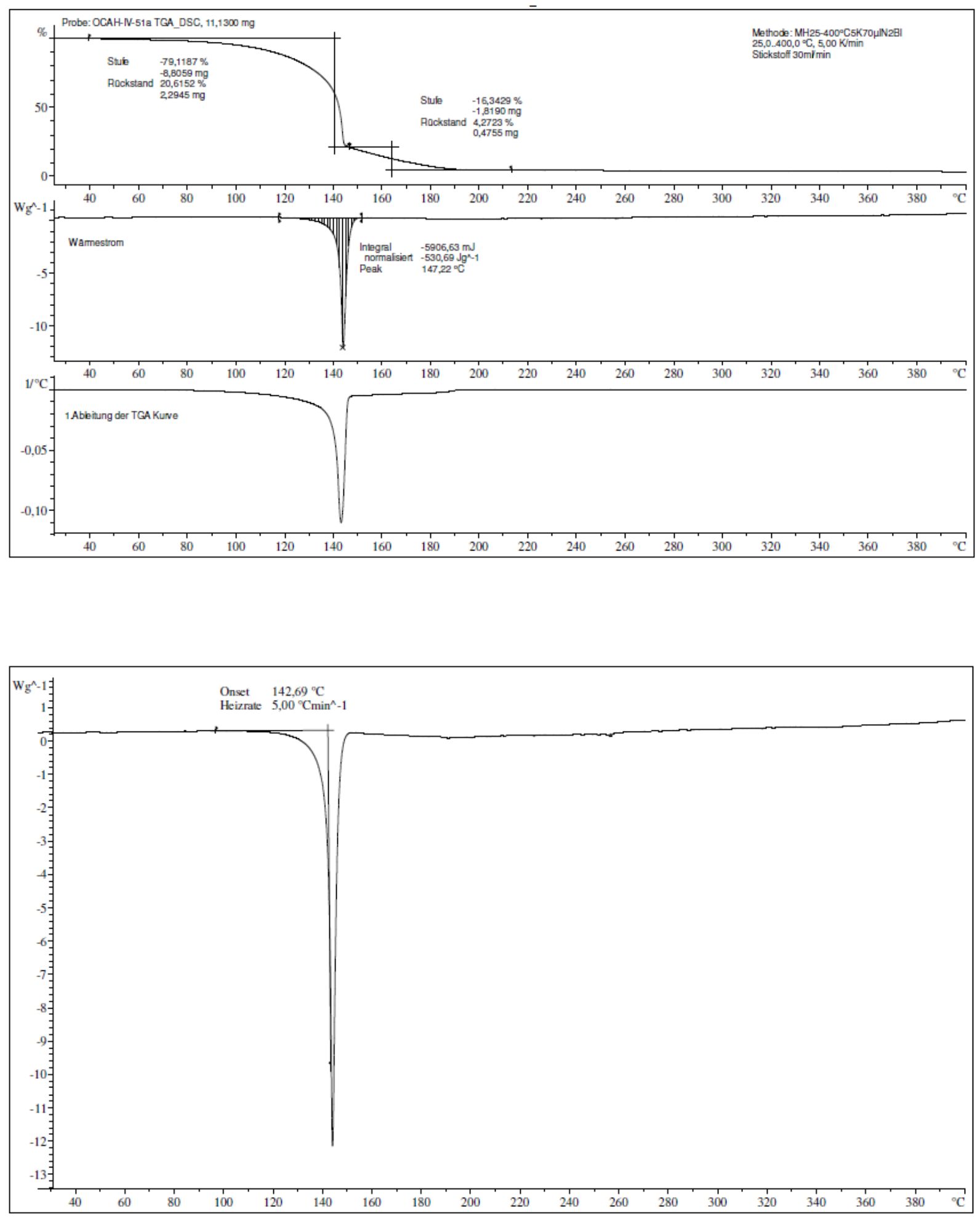

74 
tert-butyl 2,2-diazido-3-oxo-3-phenylpropanoate (2m)<smiles>CCCCOC(=O)C([NH3+])([NH3+])C(=O)c1ccccc1</smiles>
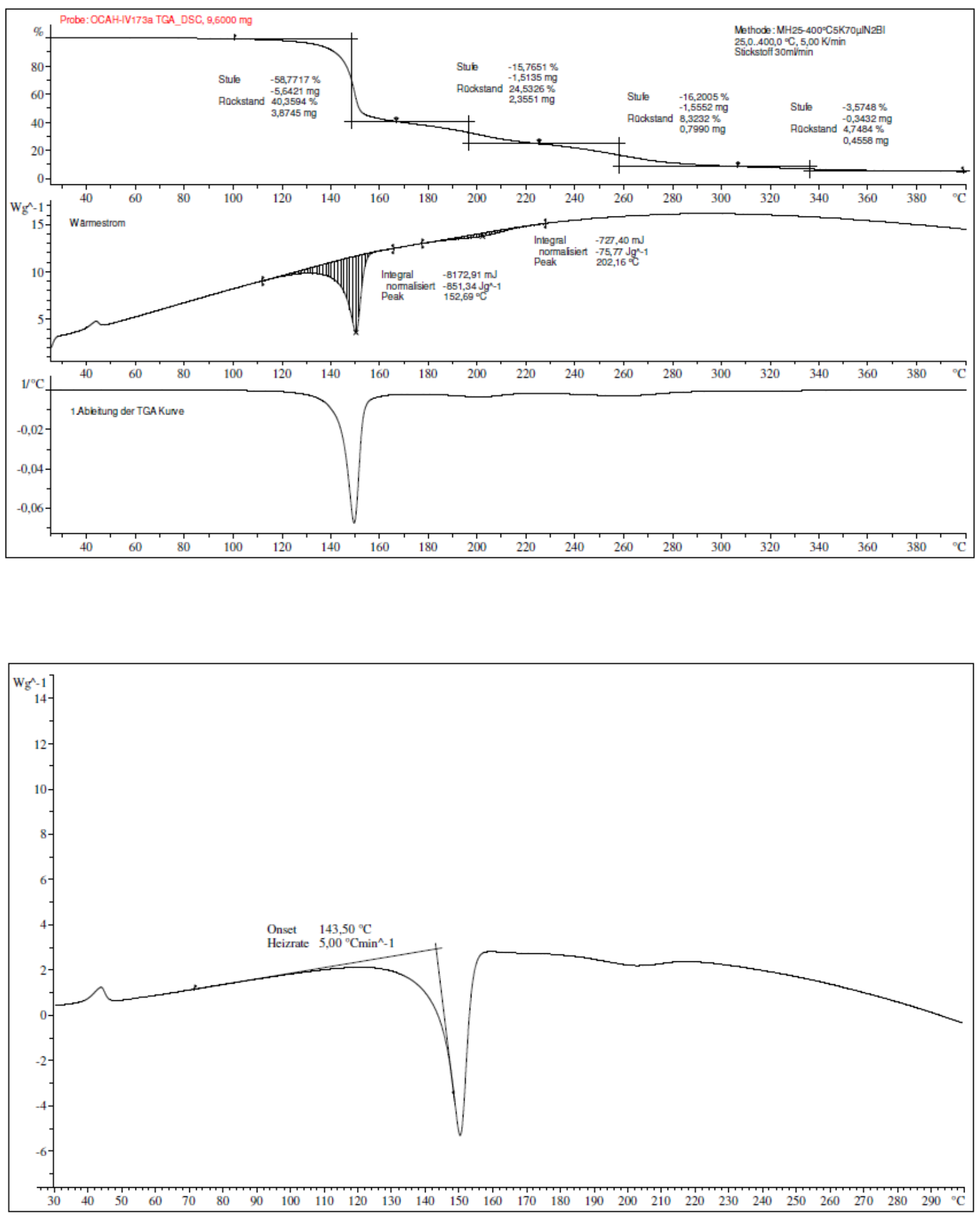


\section{3,3-diazido-5,5-dimethylhexane-2,4-dione (2ac)}
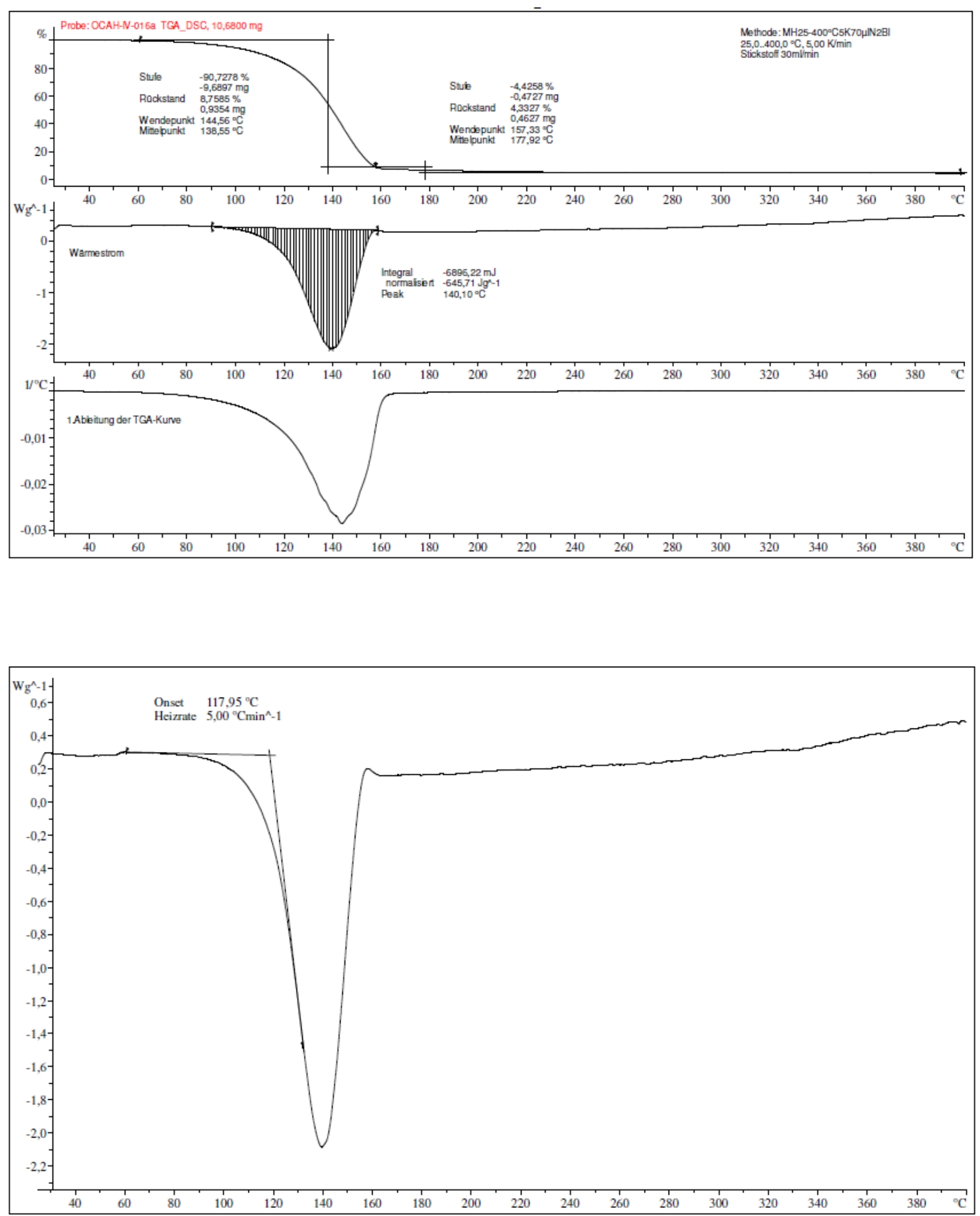
tert-butyl 2,2-diazidoacetate
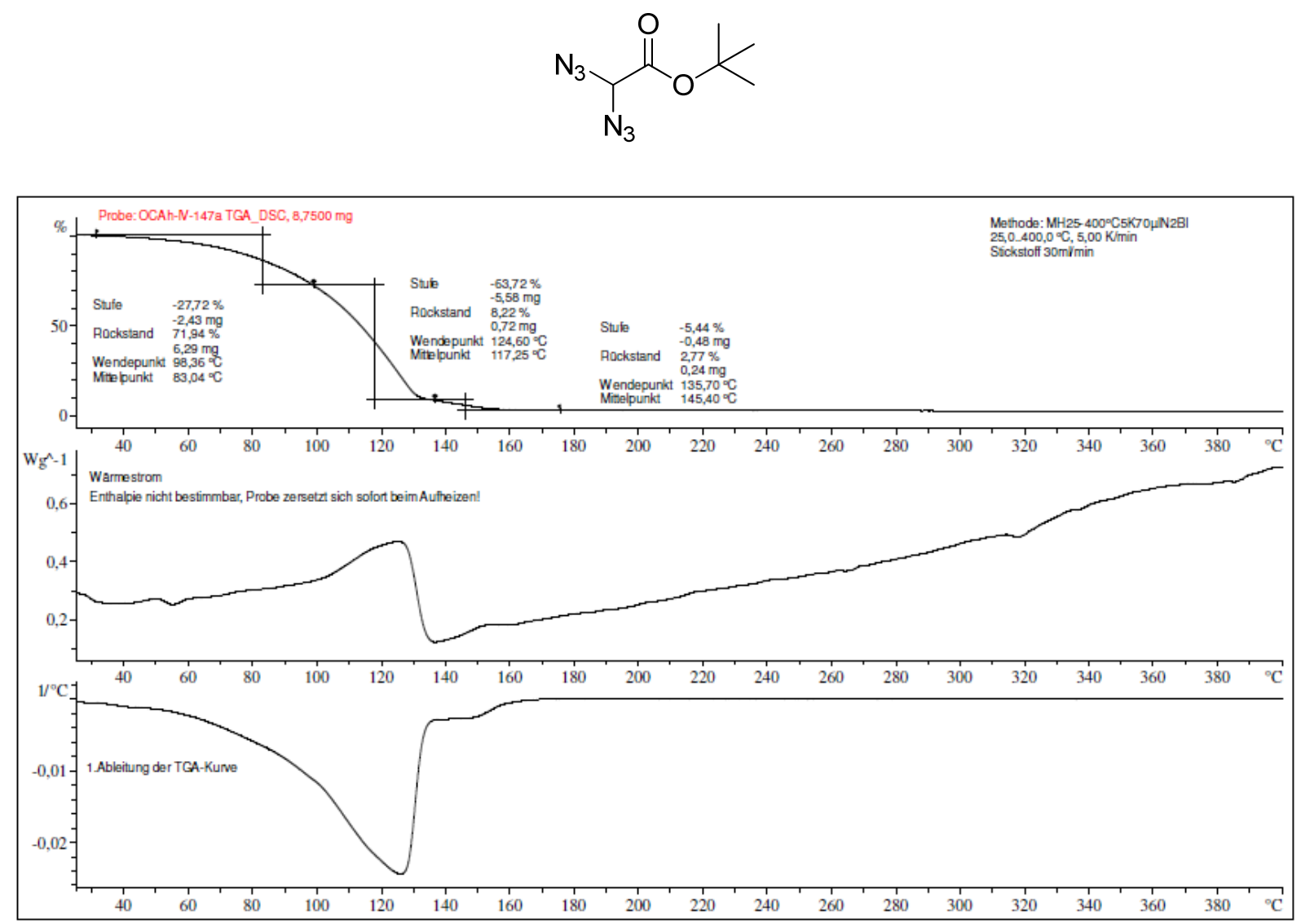


\section{Chiral HPLC measurements}

\section{(S)-tert-butyl 2,2-diazido-4-((tert-butoxycarbonyl)amino)-3-oxopentanoate (2z)}
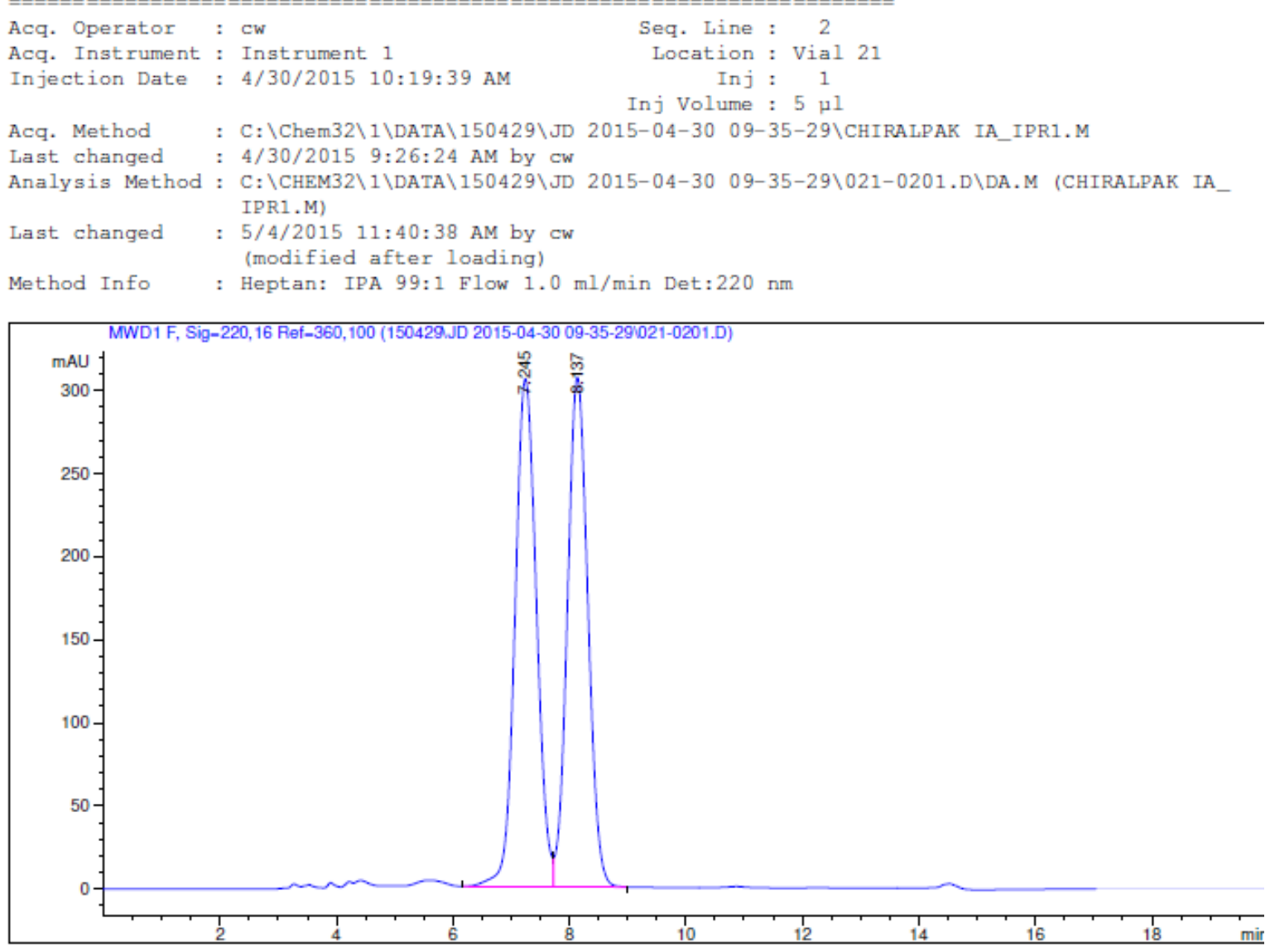

Area Percent Report

$\begin{array}{lll}\text { Sorted By } & : & \text { Signal } \\ \text { Multiplier } & : & 1.0000\end{array}$

Dilution : 1.0000

Use Multiplier \& Dilution Factor with ISTDs

Signal 1: MWD1 F, Sig=220, 16 Ref $=360,100$

\begin{tabular}{|c|c|c|c|c|c|c|}
\hline $\begin{array}{c}\text { Peak } \\
\#\end{array}$ & $\begin{array}{l}\text { RetTime } \\
\text { [min] }\end{array}$ & Type & $\begin{array}{c}\text { Width } \\
\text { [min] }\end{array}$ & $\begin{array}{c}\text { Area } \\
{\left[m A U^{*} s\right]}\end{array}$ & $\begin{array}{l}\text { Height } \\
\text { [mAU] }\end{array}$ & $\begin{array}{c}\text { Area } \\
8\end{array}$ \\
\hline 1 & 7.245 & vV & 0.3986 & 7817.29102 & 305.44080 & 51.1259 \\
\hline 2 & 8.137 & VB & 0.3826 & 7472.98047 & 306.33417 & 48.8741 \\
\hline otal & : & & & 1. $52903 \mathrm{e}^{4}$ & 611.77496 & \\
\hline
\end{tabular}


Acq. Operator : cw

Acq. Instrument : Instrument 1

Injection Date : 4/30/2015 1:15:22 PM

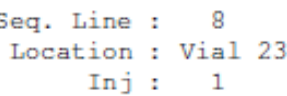

Seq. Line : 8

Location : Vial 23$$
\text { In] : } 1
$$

Inj Volume : $5 \mu 1$

Acq. Method : C: \Chem32\1\DATA \150429\JD 2015-04-30 09-35-29\CHIRALPAK IA_IPR1.M

Last changed : 4/30/2015 9:26:24 AM by cw

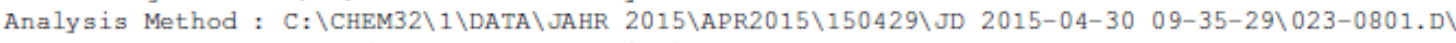

DA.M (CHIRALPAK IA_IPR1.M)

Last changed : 10/7/2015 1:15:08 PM by ip

Method Info: Heptan: IPA 99:1 Flow $1.0 \mathrm{ml} / \mathrm{min}$ Det:220 $\mathrm{nm}$

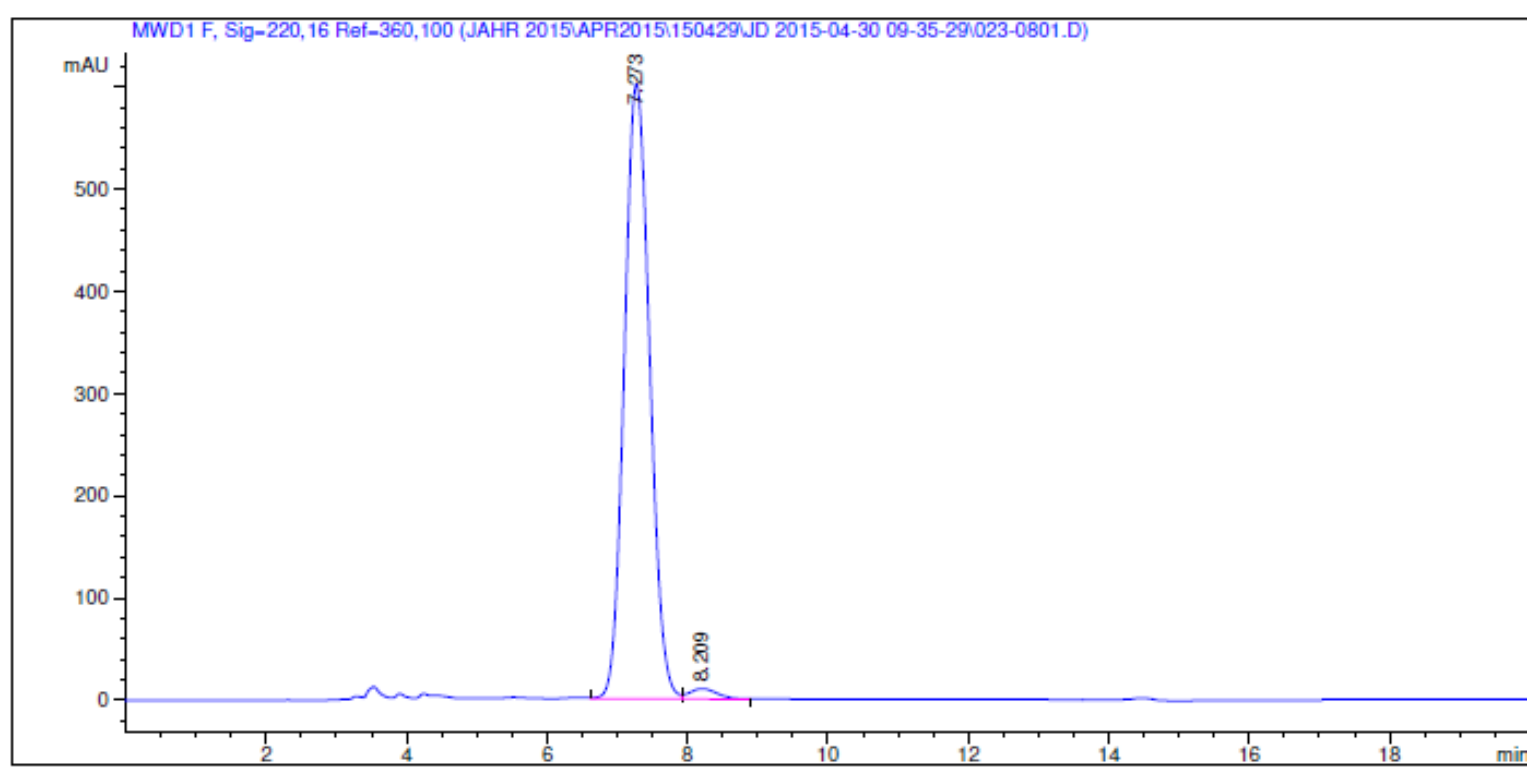

Area Percent Report

$\begin{array}{lll}\text { Sorted By } & : & \text { Signal } \\ \text { Multiplier } & : & 1.0000 \\ \text { Dilution } & : & 1.0000\end{array}$

Use Multiplier \& Dilution Factor with ISTDs

Signal 1: MWD1 F, Sig=220, 16 Ref $=360,100$

\begin{tabular}{|c|c|c|c|c|c|c|}
\hline $\begin{array}{c}\text { Peak } \\
\#\end{array}$ & $\begin{array}{c}\text { RetTime } \\
\text { [min] }\end{array}$ & Type & $\begin{array}{c}\text { Width } \\
\text { [min] }\end{array}$ & $\begin{array}{c}\text { Area } \\
{\left[\mathrm{MAU}^{*} \mathrm{~s}\right]}\end{array}$ & $\begin{array}{l}\text { Height } \\
\text { [mAU] }\end{array}$ & $\begin{array}{c}\text { Area } \\
\&\end{array}$ \\
\hline 1 & 7.273 & vV & 0.3947 & 1. $50928 \mathrm{e}^{4}$ & 601.51489 & 98.1668 \\
\hline 2 & 8.209 & VB & 0.4173 & 281.84277 & 10.17197 & 1.8332 \\
\hline otal & : & & & 1. $53747 \mathrm{e} 4$ & 611.68687 & \\
\hline
\end{tabular}

$\star \star \star$ End of Report $\star * \star$ 
(S)-tert-butyl 2,2-diazido-4-((tert-butoxycarbonyl)amino)-6-methyl-3-oxoheptanoate (2z)
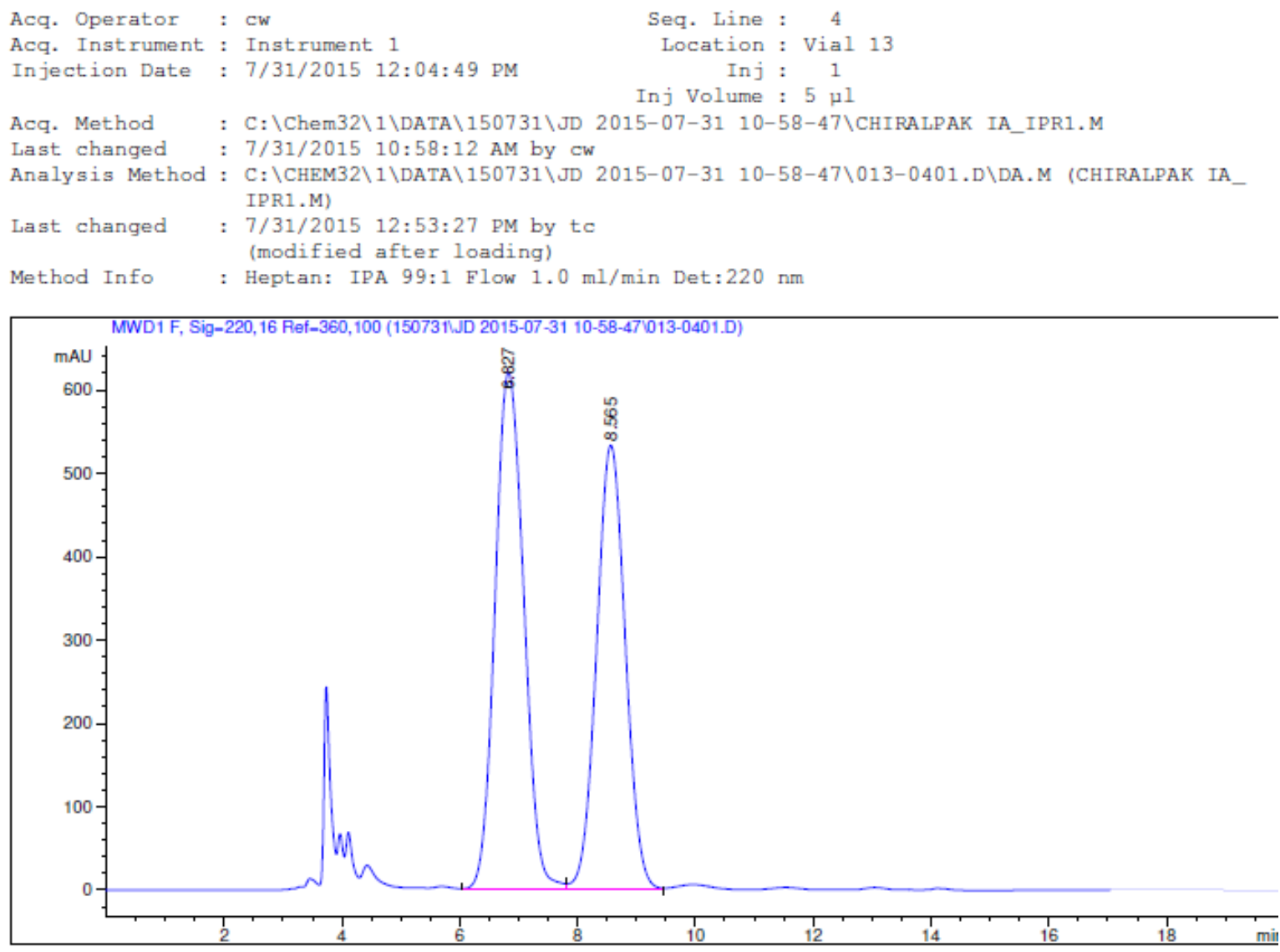

Area Percent Report

$\begin{array}{lll}\text { Sorted By } & : & \text { Signal } \\ \text { Multiplier } & : & 1.0000 \\ \text { Dilution } & : & 1.0000\end{array}$

Use Multiplier \& Dilution Factor with ISTDs

Signal 1: MWD1 F, Sig=220, 16 Ref $=360,100$

\begin{tabular}{|c|c|c|c|c|c|c|}
\hline $\begin{array}{c}\text { Peak } \\
\#\end{array}$ & $\begin{array}{l}\text { RetTime } \\
\text { [min] }\end{array}$ & Type & $\begin{array}{c}\text { Width } \\
\text { [min] }\end{array}$ & $\begin{array}{c}\text { Area } \\
{\left[\mathrm{mAU}^{\star} \mathrm{s}\right]}\end{array}$ & $\begin{array}{l}\text { Height } \\
\text { [mAU] }\end{array}$ & $\begin{array}{c}\text { Area } \\
\&\end{array}$ \\
\hline 1 & 6.827 & VV & 0.5401 & 2. $10869 \mathrm{e} 4$ & 621.04010 & 53.7884 \\
\hline 2 & 8.565 & VV & 0.5323 & 1. $81165 \mathrm{e} 4$ & 533.37750 & 46.2116 \\
\hline
\end{tabular}

Totals : $\quad 3.92034 \mathrm{e} 4 \quad 1154.41760$ 
Acq. Operator : ip

Acq. Instrument : Instrument 1

Injection Date : 8/4/2015 10:03:38 AM

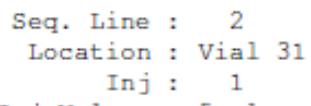

Inj Volume : 5 pl

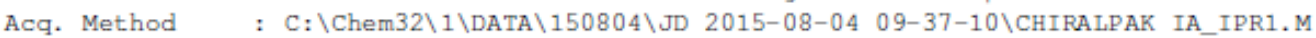

Last changed : 8/4/2015 9:28:15 AM by tc

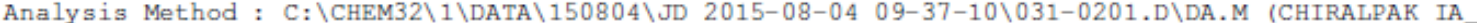
IPR1.M)

Last changed : 8/4/2015 11:51:14 AM by ip

Method Info : Heptan: IPA 99:1 Flow $1.0 \mathrm{ml} / \mathrm{min}$ Det:220 $\mathrm{nm}$

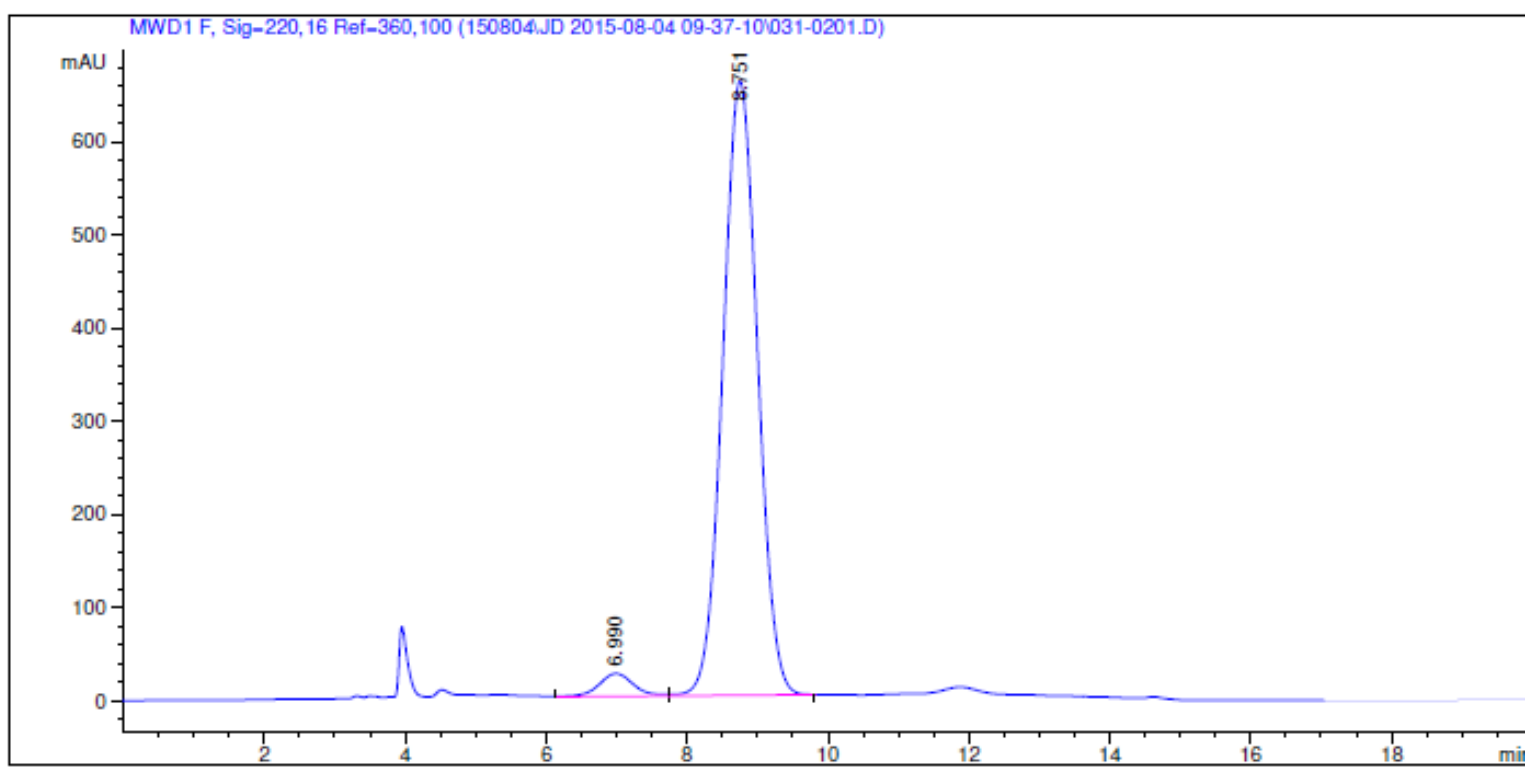

Area Percent Report

$\begin{array}{lll}\text { Sorted By } & : & \text { Signal } \\ \text { Multiplier } & : & 1.0000 \\ \text { Dilution } & : & 1.0000\end{array}$

Use Multiplier \& Dilution Factor with ISTDs

Signal 1: MND1 F, Sig=220, 16 Ref $=360,100$

\begin{tabular}{|c|c|c|c|c|c|c|}
\hline $\begin{array}{c}\text { Peak } \\
\#\end{array}$ & $\begin{array}{l}\text { RetTime } \\
\text { [min] }\end{array}$ & Type & $\begin{array}{l}\text { Width } \\
\text { [min] }\end{array}$ & $\begin{array}{c}\text { Area } \\
{\left[m \mathrm{mU}^{*} \mathrm{~s}\right]}\end{array}$ & $\begin{array}{l}\text { Height } \\
\text { [mAU] }\end{array}$ & $\begin{array}{c}\text { Area } \\
\&\end{array}$ \\
\hline 1 & 6.990 & vV & 0.5272 & 852.52197 & 24.54588 & 3.6455 \\
\hline 2 & 8.751 & VB & 0.5330 & 2. $25328 e 4$ & 658.81732 & 96.3545 \\
\hline
\end{tabular}

Totals : $\quad 2.33854 \mathrm{e} 4 \quad 683.36320$

\section{$\star * *$ End of Report $* * *$}

